\title{
CATALOGUE
}

\section{OR THE}

\section{STAMPS, ENVYZLOPES,}

WRAPPERS \& POSTAL CAROS

\author{
OF: \\ MEX I C O
}




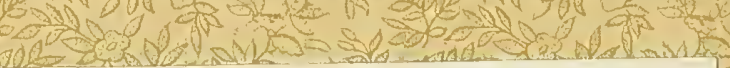




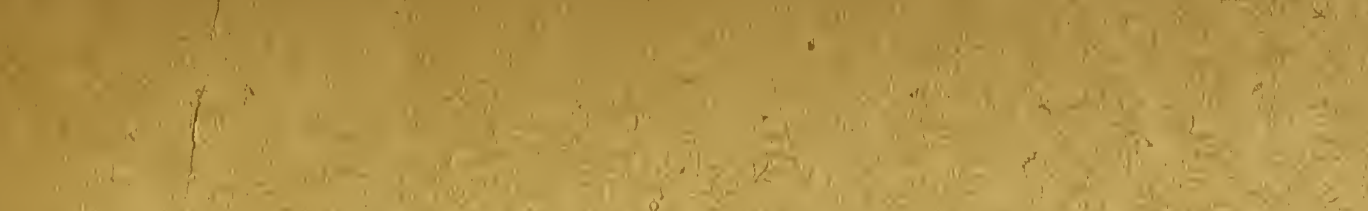

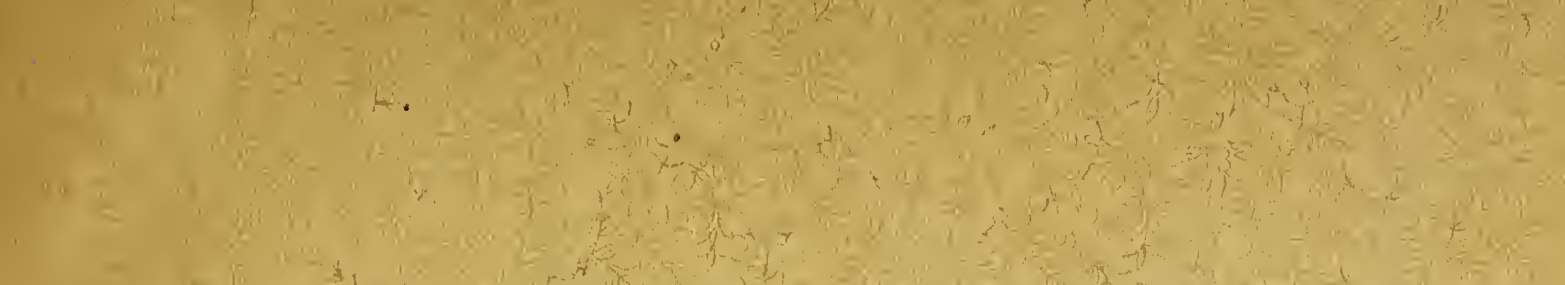

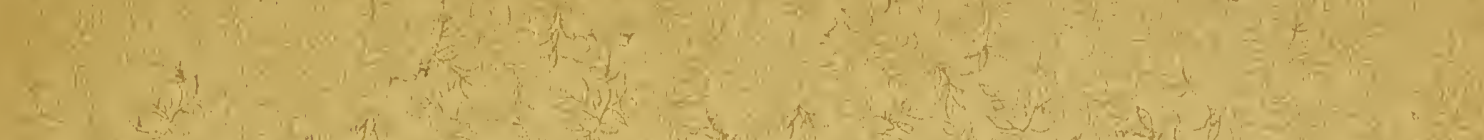

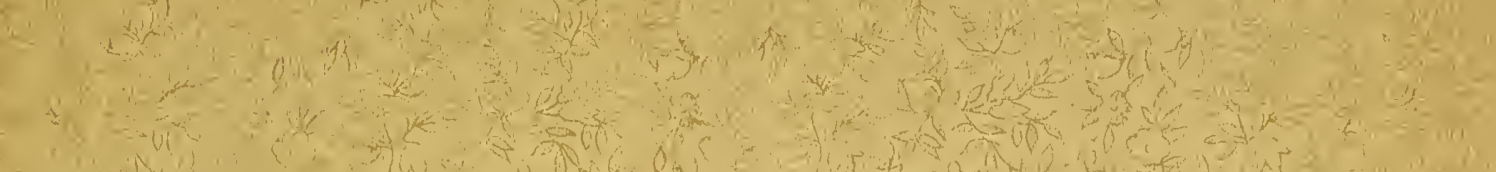
10 (n) (1) (n)

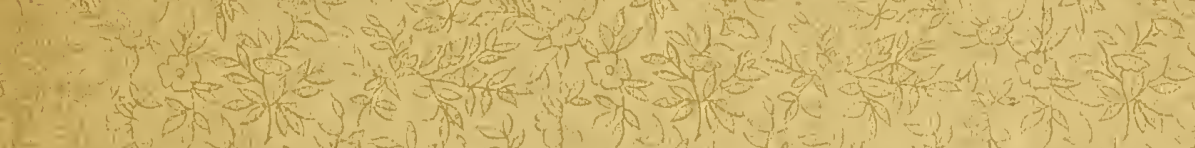

a d

$-1$

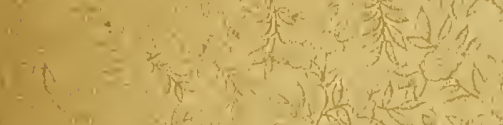

$$
\text { 3. }
$$

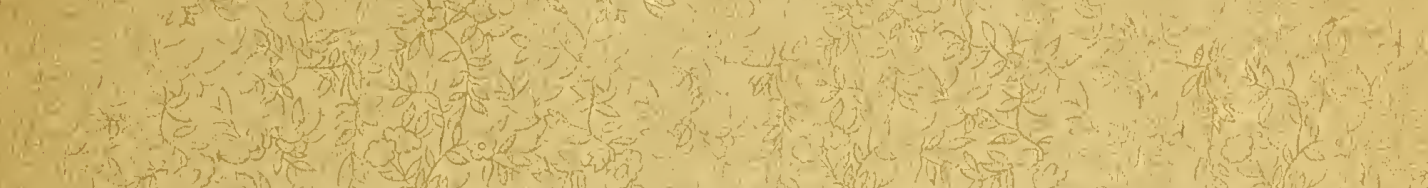

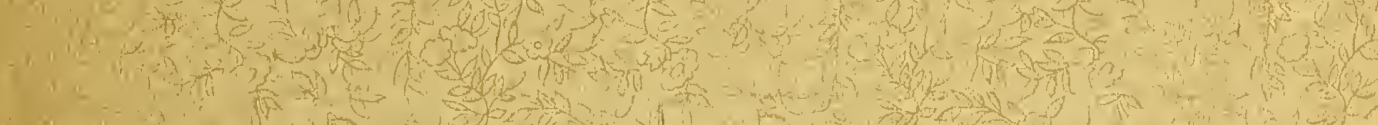
(1) (-

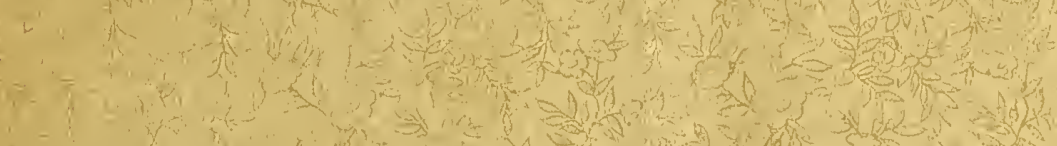

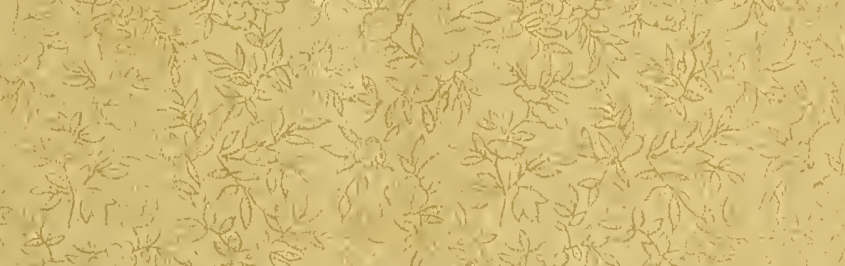






\section{.}





\title{
CATALOGUE
}

OF THE

\section{Stamps, Envelopes, Wrappers and Postal Cards}

$\mathrm{OF}$

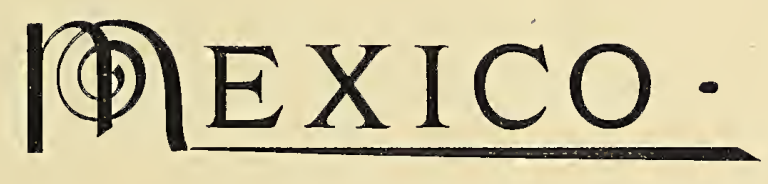

Including the Provisional Issues of

\section{Campeche, Chiapas, Guadalajara, Etc.}

\author{
HENRY COLLIN W HENRY L. CALMAN,
}

With the collaboration of Mr. Albert E. Lawrence.

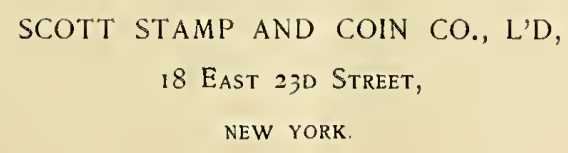





\section{N D E X}

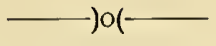

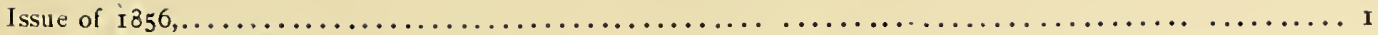

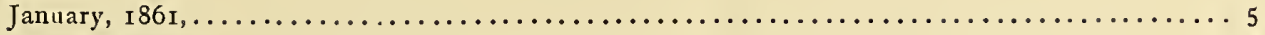

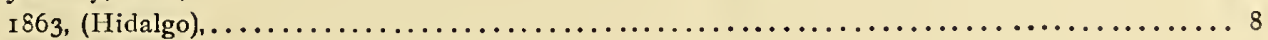

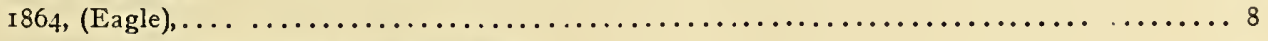

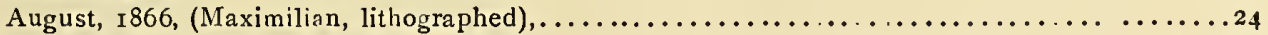

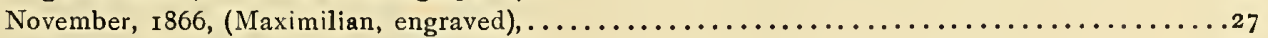

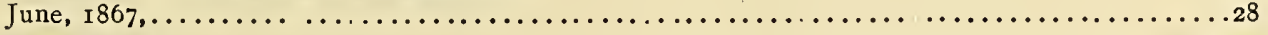

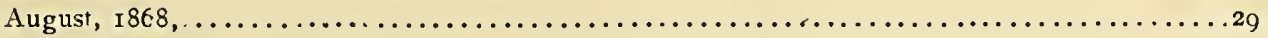

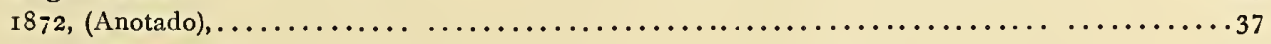

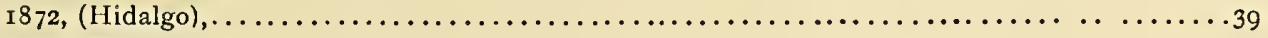

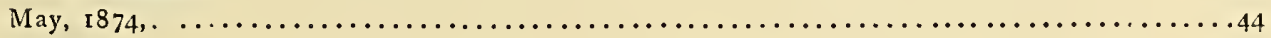

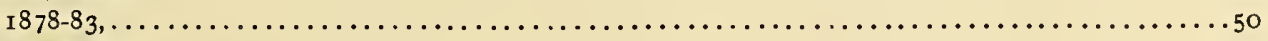

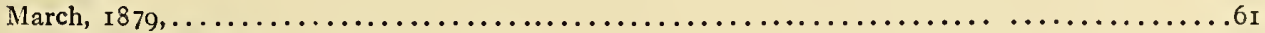

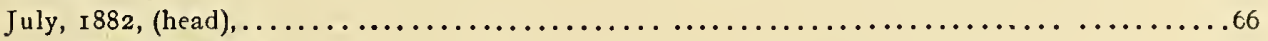

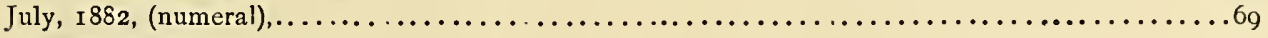

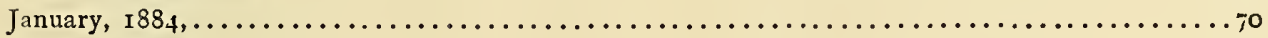

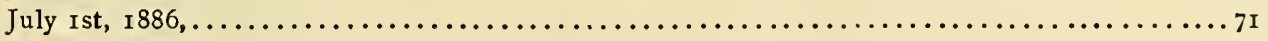

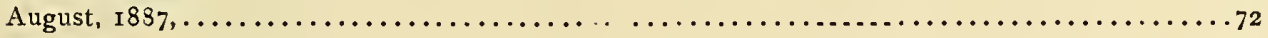

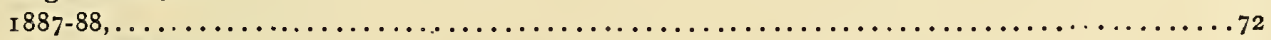

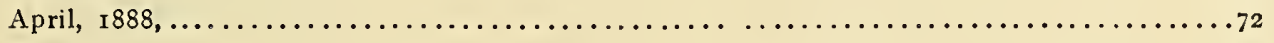

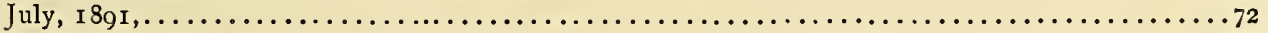

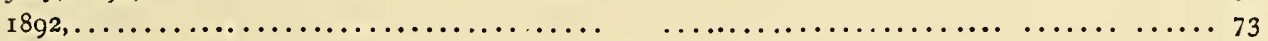

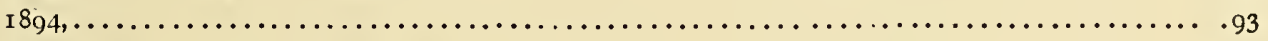

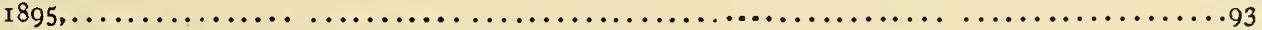

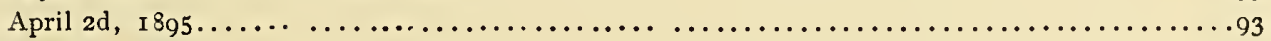

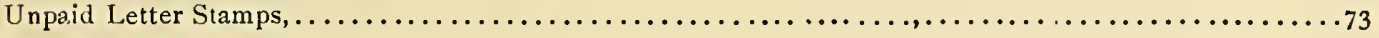

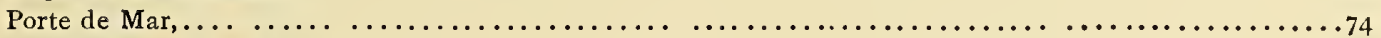

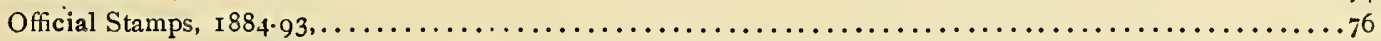

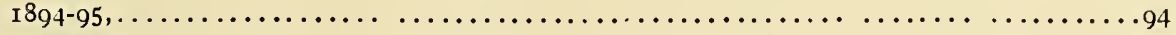

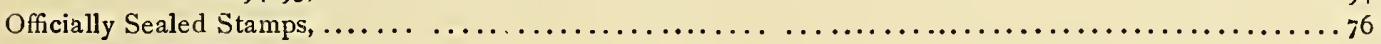

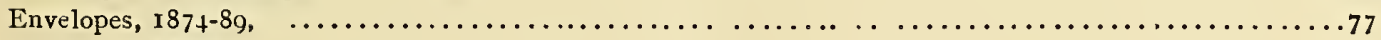

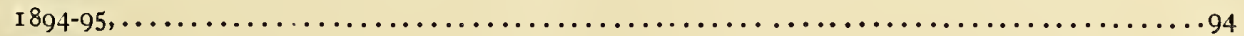

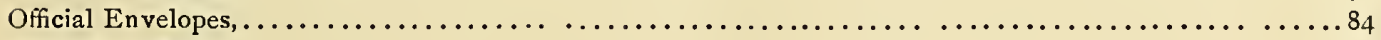

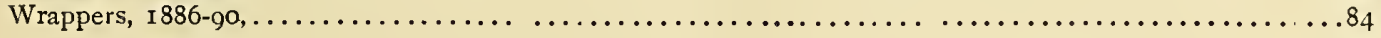

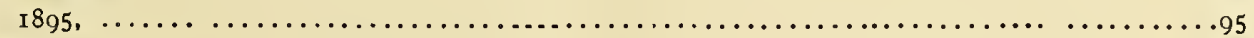

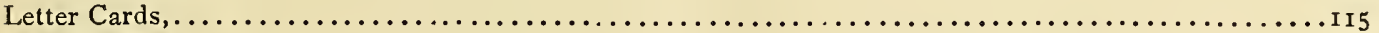

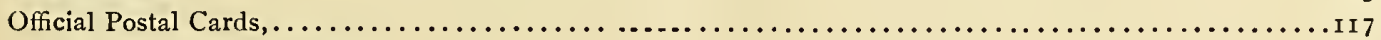

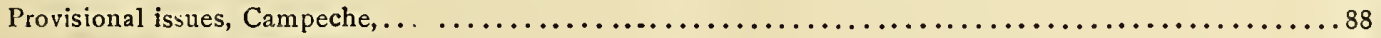

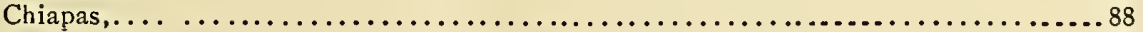

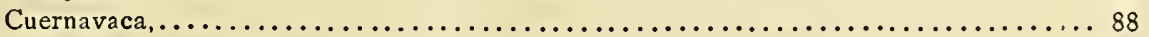

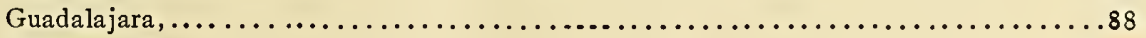

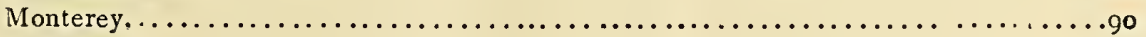

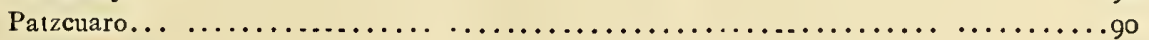

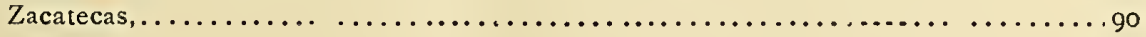

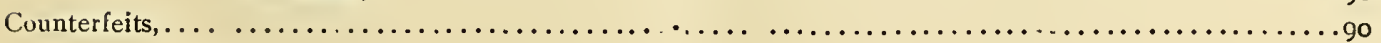






\section{MEXICO.}

Prepared with the collaboration of Mr. Albert E. Lawrence.

\section{Currency : 8 Reales $=100^{\top}$ Centavos $=$ I Peso $=\$ 0.60$ U. S. Currency}

August, 1856 .

Head of Hidalgo, engraved on white wove paper, varying in thickness. The sheets of the stamps of this issue were surrounded by a thin line of the same color as the stamps. On some of the sheets this frame is formed by a double line instead of a single line either at top or at bottom. Size $191 / 2 \times 25 \mathrm{~mm}$.

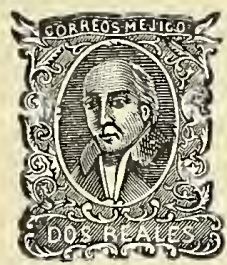

I. Without surcharge.
$1 / 2$ (Medio) $r$ blue
$1 / 2$ (Medio) r pale blue
$1 / 2$ (Medio) $\mathrm{r}$ dark blue
I (un) $r$ orange
I (un) $r$ yellow
I (un) $r$ orange red to
2 (dos) $r$ green
2 (dos) $r$ pale green
2 (dos) $\mathrm{r}$ dark green
2 (dos) $\mathrm{r}$ blue green
2 (dos) $r$ yellow green
2 (dos) $\mathrm{r}$ emerald green
4 (cuatro) r red
4 (cuatro) $r$ brown red
8 (ocho) r lilac
8 (ocho) r red lilac

II. Surcharged with the name of the postal district.

$I^{\circ}$ White wove paper, varying in thick-

$1 / 2$ (Medio) $\mathrm{r}$ blue

$1 / 2$ (Medio) $r$ pale blue

$1 / 2$ (Medio) $\mathrm{r}$ dark blue

I (un) $r$ orange

I (un) $r$ yellow

I (un) $\mathrm{r}$ orange red

2 (dos) $r$ green

2 (dos) r pale green

2 (dos) $r$ dark green

2 (dos) $r$ blue green

2 (dos) $x$ yellow green

2 (dos) $r$ emerald green

4 (cuatro) $r$ red

4 (cuatro) $r$ brown red

8 (ocho) r lilac

8 (ocho) $\mathrm{r}$ red lilac

$2^{\circ}$ Vertically ribbed paper.

$1 / 2$ (Medio) $\mathrm{r}$ blue

$1 / 2$ (Medio) $r$ pale blue

$1 / 2$ (Medio) $\mathrm{r}$ dark blue

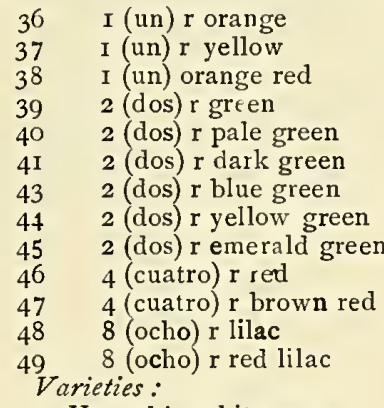

a. Very thin white paper.

504 (cuatro) $r$ pale red

b. Laid paper.

5 I 4 (cuatro) r red

c. Error of color.

522 (dos) $\mathrm{r}$ rose

d. Lower part of 4 reales stamp printed over the centre of I real stamp.

53 Ir yellow

e. 8 reales stamps cut in eight parts, each part being used as I real.

54 Ir lilac ( $1 / 8$ of 8 reales used as 1 real

f. 2 reales stamps cut vertically in two, each half being used as I real.

55 Ir green (half of 2 reales used as I real), left half

56 Ir green (half of 2 reales used as I real), right half

g. 2 reales stamps cut horizontally in two, each half being used as I real.

57 Ir green (half of 2 reales used as I real), upper half

58 Ir preen (half of 2 reales used as I real), lower half.

h. 2 reales stamps cut diagonally in two, each half being used as I real.

59 Ir green (half of 2 reales used as I real), upper right half

60 Ir green (half of 2 reales used as I real), lower right half

6I Ir green (half of 2 reales, used as I real), upper left half

62 Ir green (half of 2 reales used as 1 real), lower left half

i. 4 reales stamps cut in quarters, each quarter being used as I real.

63 Ir red (one-fourth of 4 reales used as I real), upper right fourth

64 Ir red (one-fourth of 4 reales used as I real), lower right fourth

65 Ir red (one-fourth of 4 reales used as I real), upper left fourth

66 Ir red (one-fourth of 4 reales used as I real), lower left fourth

k. 4 reales stamps cut vertically in two each half being"used as" 2 reales. 
$672 \mathrm{r}$ red (half of 4 reales used as 2 reales), right half

$682 \mathrm{r}$ red (half of 4 reales used as 2 reales), left half

1. 4 reales stamps cut horizontally in two, each half being used as 2 reales.

$692 \mathrm{r}$ red (half of 4 reales used as 2 reales), upper half

$70 \quad 2 r$ red (half of 4 reales used as 2 reales), lower half

m. 4 reales stamps cut diagonally in two, each half being used as 2 reales.

$7 \mathrm{I} 2 \mathrm{r}$ red (half of 4 reales used as 2 reales), upper right half

72 2r red (lialf of 4 reales used as 2 reales), lower right half

$732 \mathrm{r}$ red (half of 4 reales used as 2 reales), upper left half

74 2r red (half of 4 reales used as 2 reales), lower left half

n. 8 reales stamps cut in quarters, each quarter being used as 2 reales.

75 2r lilac (one-fourth of 8 reales used as 2 reales), upper right quarter

762 2r lilac (one-fourth of 8 reales used as 2 reales), lower right quarter 2r lilac (one-fourth of 8 reales used as 2 reales), upper left quarte

78 2r lilac (one-fourth of 8 reales used as 2 reales), lower left quarter

o. 8 reales stamps cut vertically in two each half being used as 4 reales.

$794 \mathrm{r}$ lilac (half of 8 reales used as 4 reales), right half

So $4 \mathrm{r}$ lilac (half of 8 reales used as 4 reales), left half

p. 8 reales stamps cut horizontaliy in two, each half being used as 4 reales.

8I $4 \mathrm{r}$ lilac (half of 8 reales used as 4 reales), upper half

$824 \mathrm{r}$ lilac (half of 8 reales used as 4 reales), lower half

q. 8 reales stamps cut diagonally in two, each half being used as 4 reales.

$834 \mathrm{r}$ lilac (half of 8 reales used as 4 reales), upper right half

$844 \mathrm{r}$ lilac (half of 8 reales used as 4 reales), lower right half

854 4r lilac (half of 8 reales used as 4 reales), upper left half

$864 \mathrm{r}$ lilac (half of 8 reales used as 4 reales), lower left half

District Names found on the Issue of I856.

\begin{tabular}{|c|c|}
\hline District. & Meas. in $\mathrm{mm}$. \\
\hline ACAPULCO. & I $81 / 2 \times 2$; bet. periods \\
\hline AGUACALTES & $2 \mathrm{Ix}_{3}$ \\
\hline A.PAM & $9 \times 2$ \\
\hline “ & $9 \times 2$; sur'ge in blue \\
\hline - CAMPECHE. & $\begin{array}{l}\text { I } 8 \times 2 \text {; bet. periods } \\
\text { I } 9 \times 2 \text {; bet. periods, } \\
\text { sur'ge in red }\end{array}$ \\
\hline C. Bravos & (Caillebotte) \\
\hline CORDOVA & I $81 / 2 \times 2$; bet. periods \\
\hline $\begin{array}{l}\text { CORDOVA. } \\
\text { CORDOYA. }\end{array}$ & $\begin{array}{l}\text { I } 71 / 2 \times 2 \\
\text { I } 6 \times 1 / 2 ; \text { bet. periods }\end{array}$ \\
\hline CORDOVA & $181 / 2 \times 2$ \\
\hline $\begin{array}{l}\text { Cordova, } \\
\text { CUAUTITLAN }\end{array}$ & $\begin{array}{l}\text { (Caillebotte); italics } \\
\text { I6x2 }\end{array}$ \\
\hline CUERNAVACA. & I $8 \times 2$; with one period \\
\hline CUERNAVACA & $171 / 2 \times 2$ \\
\hline CUERNAVACA & $\mathrm{I} 7 \mathrm{X} I \mathrm{I} / 2$ to $\mathrm{I} 3 / 4$ \\
\hline CHA LCO & I $2 \times 2$ \\
\hline - CHIA & $\begin{array}{l}\text { I } 6 \times 2 \text {, bet. periods } \\
\text { I } 6 \times 2 \text {; bet. periods, } \\
\text { sur'ge in blue }\end{array}$ \\
\hline - CHI HUAHUA• & $201 / 2 \times 2$; bet. periods \\
\hline CHIHUAHUA. & $\begin{array}{l}\text { I8 } 1 / 2 \times 1 \frac{1}{2} ; \text { with one } \\
\text { period }\end{array}$ \\
\hline - DURANGO. & I7x2 ; bet. periods \\
\hline GUADALAJARA & $191 / 2 \times 21 / 4$ \\
\hline '(广UADALAJARA" & $201 / 2 \times 2$; bet. periods \\
\hline GUADA $\underset{" ،}{\text { LAJARA }}$ & $\begin{array}{l}2 \mathrm{Ix} 2 \text {; with one period } \\
2 \mathrm{I} \times 2\end{array}$ \\
\hline GUADALAJARA & $\operatorname{Ig} 3 / 4 \times 2$ \\
\hline GUAD ALA.JARA & I9x2 $1 / 4$ \\
\hline GCADA LAJA A & $201 / 2 \times 11 / 4$ \\
\hline GUADALAJARA & I $9 \times 1 / 4$ to $23 / 4$ \\
\hline GUADALAJARA & $20 \times 1 / 2$ to $31 / 4$ \\
\hline GUADALA JARA & $20 \times 21 / 2$ to $I / / 4$ \\
\hline GUANAJUTO. & $23 \times 3$; with one period \\
\hline - GUANAJ UATO• & I $91 / 2 \times 2$; bet. periods \\
\hline GUANAJ UATO & I $8 \times I 3 / 4$ \\
\hline GUANAJUATO & I9 $1 / 4$ X2 to 3 ; bet.periods \\
\hline 'GUANAJUATO' & $\begin{array}{l}\text { I9 } 1 / 4 \times 1 / 2 \text { to } 2 z / 1 ; \text { bet. } \\
\text { periods }\end{array}$ \\
\hline GUANAJUATO & I $8 \times I^{1 / 2}$ to I $3 / 4$ \\
\hline 'HERMOSIIIO. & $\begin{array}{l}2 \mathrm{IX} 2 \text {; bet. periods } \\
\mathrm{I} 6 \mathrm{x} / 2 \mathrm{x} 2\end{array}$ \\
\hline
\end{tabular}

\begin{tabular}{|c|c|}
\hline District. & Meas. of $\mathrm{mm}$. \\
\hline I. DEL CARMEN. & $\begin{array}{l}2 \mathrm{Ix} 2 \text {; bet. periods, } \\
\text { sur'ge in blue }\end{array}$ \\
\hline IXTLAHUACA & I $9 \times 13 / 4$ \\
\hline IXTLAHUACA & $\mathrm{I} 9 \times 3$ to $\mathrm{I} 3 / 4$ \\
\hline$\cdot \mathbf{J A L A P A}$ & I $41 / 2 \times 2$; bet. periods \\
\hline LAGOS & $\operatorname{I} \operatorname{IX} 2 \mathrm{I} / 2$ \\
\hline LFRMA & $12 \times 21 / 2$ \\
\hline MARAVATIO & $17 \times 2$ \\
\hline MAZATLAN & I $7 \times 2$ \\
\hline MAZATLAN' & I $7 \times 2$; bet. periods \\
\hline MERIDA & I $5 \times 2$ \\
\hline MEXICO & $I 6 \times 23 / 4$ \\
\hline MI [A]COCD & $141 / 2 \times 21 / 4$ \\
\hline 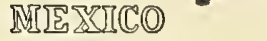 & $151 / 2 \times 23 / 4$ \\
\hline MI政Ico & $14 \times 21 / 2$ to $13 / 4$ \\
\hline MEXICO & $171 / 2 \times 21 / 2$ \\
\hline $\mathrm{M} \mathbb{E _ { 0 }} \times \mathbb{C} \mathbb{C}$ & $16 \times 2$ \\
\hline$\rightarrow \mathrm{MEXICO}$ & $23 \times 2$; bet. two dashes \\
\hline$M E X I C O$ & I $4 \times 2$ \\
\hline MIEXIICOO & $\mathrm{I} 6 \times 2$ \\
\hline M EXICO & $\mathrm{I} 4 \times 2 \mathrm{I} / 4$ \\
\hline M E XICO & $14 \times 2$ \\
\hline MEXICO & I $33 / 4 \times 2$ to $31 / 3$ \\
\hline MONTERREY• & $20 \times 13 / 4$; bet. periods \\
\hline MORELIA & $131 / 2 \times 2$ \\
\hline -MORELIA. & I $5 \mathrm{I} / 4 \mathrm{xI} 3 / 4$; bet. periods \\
\hline $\begin{array}{l}\text { OAJACA } \\
. \mathrm{OAJACA}\end{array}$ & I $4 \times 2 / 2 \times 2$; bet. periods \\
\hline - OAJACA. & I $5 \times 2$; bet. periods \\
\hline $\begin{array}{l}\text { OAJACA } \\
\text { OAJACA. }\end{array}$ & $\begin{array}{l}\text { I } 5 \times 4 \text { to } 2 \text {; bet. periods } \\
\text { I } 5 \times 2 \text {; bet. periods }\end{array}$ \\
\hline $\begin{array}{l}\text { ORIZAVA. } \\
\text { ORIZAVA }\end{array}$ & I $5 \times \mathrm{XI} / 2$; bet. periods \\
\hline PACHUCA & $\begin{array}{l}13 \times 1 / 2 \\
\text { I } 3 \times I 1 / 2\end{array}$ \\
\hline PUEBIA & $\mathrm{I} 3 \times 23 / 4$ \\
\hline PUEBLA & $12 \times 2$ \\
\hline - PUEBLA. & I $4 / 2 \times 2$; bet. periods \\
\hline $\begin{array}{l}\text { PUEBLA } \\
\text { •PUEBLA• }\end{array}$ & $\begin{array}{l}\text { I } 4 \times 2 \frac{1}{4} \\
\text { I } 4 \times 2 ; \text { bet. periods }\end{array}$ \\
\hline -PUEBLA. & I I $1 / 2 \times 2$; bet. periods \\
\hline UEBIA & I IXI $1 / 2$; bct. periods \\
\hline & I $3 \times I / 2 ; w i t$ \\
\hline
\end{tabular}




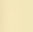


MEXICO

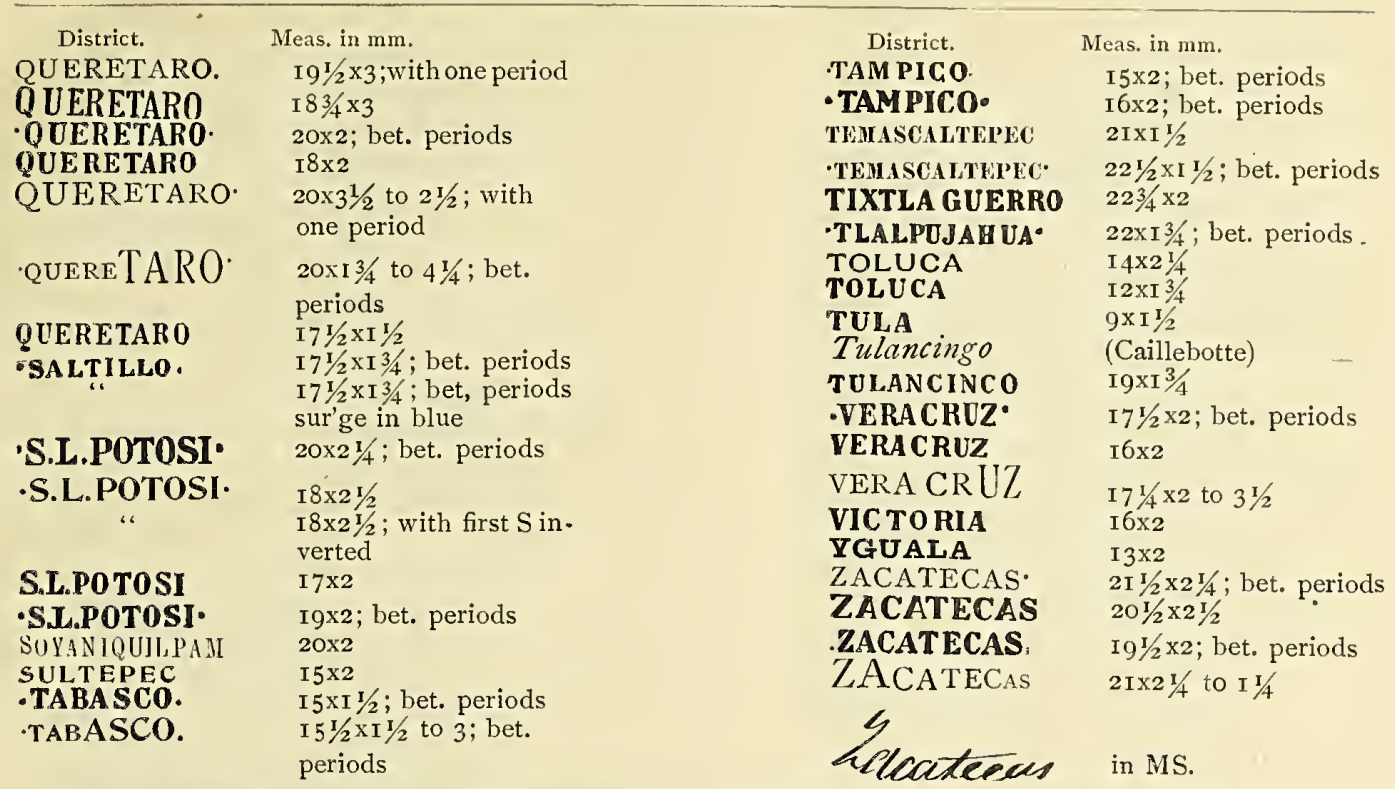

Names of postal districts found surcharged two or more times on the stamps of this issue.

\begin{tabular}{|c|c|c|c|c|c|}
\hline $\begin{array}{l}\text { District. } \\
\text { Cordova } \\
\text { “. }\end{array}$ & $\begin{array}{l}\text { Value. } \\
1 / 2 \mathrm{r} \\
2 \mathrm{r}\end{array}$ & $\begin{array}{l}\text { Color. } \\
\text { blue } \\
\text { green }\end{array}$ & $\begin{array}{l}\text { Meas. of Sur. } \\
17 \frac{1}{16} \times 2\end{array}$ & $\begin{array}{l}\text { Color. } \\
\text { black } \\
\text { “، }\end{array}$ & $\begin{array}{l}\text { Times Surcharged. } \\
\text { twice } \\
3 \text { times }\end{array}$ \\
\hline Chihuahua & $1 / 2 \mathrm{r}$ & blue & $201 / 2 \times 2$ & " & $\begin{array}{l}3 \text { twices } \\
\text { twice }\end{array}$ \\
\hline Guadalajara & Ir & orange & $201 / 2 \times 11 / 4$ & “ & $"$ \\
\hline Mazatlan & Ir & orange & $17 \times 2$ & “" & :" \\
\hline “" & $2 \mathrm{r}$ & green & $"$ & “ & "“" \\
\hline Mexico & Ir & orange & $14 \times 2$ & “" & ، \\
\hline Morelia & $1 / 2 \mathrm{r}$ & blue & $131 / 2 \times 2$ & “" & “" \\
\hline " & Ir & orange & " & " & “" \\
\hline ". & $2 \mathrm{r}$ & green & “" & “" & " \\
\hline Pachuca & Ir & orange & $13 \times 11 / 2$ & “" & “ \\
\hline Queretaro & Ir & orange & $18 \times 2$ & “" & “ \\
\hline S. L. Potosi & $2 \mathrm{r}$ & green & $\mathrm{I} 8 \mathrm{XI} \mathrm{I} / 2$ & “" & " \\
\hline 6" & $8 r$ & lilac & $20 \times 21 / 4$ & ، & " \\
\hline Vera Cruz & Ir & orange & $171 / 2 \times 2$ & & " \\
\hline " & $2 \mathrm{r}$ & green & " & “. & " \\
\hline “" & $4 \mathrm{r}$ & red & ' & “" & “ \\
\hline “" & $2 r$ & green & “" & ". & 3 times \\
\hline Zacatecas & $2 \mathrm{r}$ & green & $191 / 2 \times 2$ & “" & twice \\
\hline
\end{tabular}

\section{Reprints.}

Although the greater part of the uncancelea stamps of this and the next issue, which have appeared recently are genuine remainders, we believe that some reprinting has been going on; there is no other explanation for the appearance of many varieties totally unknown until a year or two ago: Such as the $4 \mathrm{r} 1856$ on laid paper, 2r rose 1856 , 2r green on blue paper, and 2r black on yellow paper 1861.

Some of the stamps of the 1856 issue are found with a large imprint in black, 1/4, 1/2, I or 2 , which is considered a surcharge by some compilers. To our mind, however, there is very little doubt that these were not sur. charges, as stamps have been met with on which this so-called surcharge was partly on the letter and partly on the stamp, plainly showing that it had been impressed on the stamp after it had been used, and probably represented an unpaid tax.

January, I86I.

Same type as preceding issue, printed in black on various colored papers.

I. Without name of postal district.

Colored wove paper.

$87 \quad 1 / 2$ (Medio) r black on light brown

88 I (un) r black on green

89 I (un) r black on blue green

902 (dos) $r$ black on pink

9 I 2 (dos) $r$ black on lilac rose

924 (cuatro) $r$ black on yellow

938 (ocho) r black on red brown

II. With name of postal district.

$\mathbf{I}^{\circ}$ On colored wove paper.

$94 \quad 1 / 2$ (medio) $r$ black on light brown

95 I (un) $r$ black on green

96 I (un) $r$ black on blue green

$97 \quad 2$ (dos) $r$ black on pink

982 (dos) $r$ black on lilac rose

994 (cuatro) $\mathrm{r}$ black on yellow

IOO 8 (ocho) $\mathrm{r}$ black on red brown

$2^{\circ}$ On vertically and horizontally ribbed

IOI $\quad 1 / 2$ (Medio) $r$ black on light brown

IO2 I (un) $r$ black on green

I03 I (un) r black on blue green

I04 2 (dos) $r$ black on pink

I05 2 (dos) $\mathrm{r}$ black on lilac rose red

I06 4 (cuatro) r yellow

IO7 8 (ocho) r red brown

Varieties:

a. Perforated unofficially.

Ios 2 (dos) $r$ black on pink 
b. Error of color.

I09 I (un) r black on yellow

I IO I (un) r black on rose

I I 2 (dos) r green on dark blue

I 22 (dos) r black on yellow

c. 8 reales stamps cut in 8 parts, each part being used as $I$ real.

I 3 Ir red brown (one-eight of 8 reales)

d. 2 reales stamps cut vertically in two, each half being used as I real.

I 4 Ir rose (half of 2 reales), right half

I 5 Ir rose (half of 2 reales), left half

e. 2 reales stamps cut horizontally in two, each half being used as I real.

I 6 Ir rose (half of 2 reales), upper half

I 7 Ir rose (half of 2 reales), lower half

f. 2 reales stamps cut diagonally in two, each half being used as I real.

I 8 Ir rose (half of 2 reales), upper right half

I 9 Ir rose (half of 2 reales), lower right half

I 20 Ir rose (half of 2 reales), upper left half

I2I Ir rose (half of 2 reales), lower left half

g. 4 reales stamps cut into quarters, each quarter being used as $I$ real

I22 Ir yellow (one-fourth of 4 reales) upper right quarter

I23 Ir yellow (one-fourth of 4 reales) lower right quarter

I24 Ir yellow (one-fourth of 4 reales) upper left quarter

I25 Ir yellow (one-fourth of 4 reales) lower left quarter

h. 4 reales stamps cut vertically in two, each half being used as 2 reales.

I 26 2r yellow (half of 4 reales), right half I27 2r yellow (half of 4 reales), left half

i. 4 reales stamps cut horizontally in two, each half being used as 2 reales.

I25 2r yellow (half of 4 reale,), upper half I $292 \mathrm{r}$ yellow (half of 4 reales), lower half

j. 4 reales stamps cut diagonally in two, each half being used as 2 reales.

I30 2r yellow (half of 4 reales), upper right half

I 3 I 2r yellow (half of 4 reales), lower right half

I32 2r yellow (half of 4 reales), upper left half

I 33

2r yellow (half of 4 reales), lower left half

k. 8 reales stamps cut in quarters, each quarter being used as 2 reales.

I34 2r brown (one-fourth of 8 reales), upper right quarter

I $352 \mathrm{r}$ brown (one-fourth of 8 reales), lower right quarter

I36 2r brown (one-fourth of 8 reales), upper left quarter

I37 2r brown (one-fourth of 8 reales), lower left quarter

1. 8 reales stamps cut vertically in two, each half being used as 4 reales

I38 $4 \mathrm{r}$ brown (half of 8 reales), right half

I39 $4 \mathrm{r}$ brown (half of 8 reales), left half

m. 8 reales stamps cut horizontally in two, each half being used as 4 reales.

I40 $4 \mathrm{r}^{\mathrm{r}}$ brown (half of 8 reales), upper half
I4I 4 r brown (half of 8 reales), lower half n. 8 reales stamps cut diagonally in two, each half being used as 4 reales.

I42 $4 \mathrm{r}$ brown (half of 8 reales) upper right half

I43 4r brown (half of 8 reales), lower right half

$1444 \mathrm{r}$ brown (half of 8 reales), upper left half

I45 $4 \mathrm{r}$ brown (half of 8 reales), lower left half

o. Laid paper.

I $46 \quad 1 / 2$ (medio)r black on light brown

November, I 86 I.

Same type and impression as preceding issue.

On colored wove paper.

$I^{\circ}$ Without name of postal district.

I 47 (dos) $r$ grayish green on pink

I48 4 (cuatro) $r$ red on yellow

I49 4 (cuatro) $r$ red on orange

I50 8 (ocho) $r$ green on reddish brown

$2^{\circ}$ With name of postal district.

I5 I 2 (dos) r grayish green on pink

I 524 (cuatro) $r$ red on yellow

I 534 (cuatro) $r$ red on orange

I54 8 (ocho) $r$ green on reddish brown

Varieties :

a. 4 reales stamps cut vertically in two, each half being used as 2 reales.

I55 $2 \mathrm{r}$ red on orange (half of 4 reales), right half

I56 2r red on orange (half of 4 reales), left half

b. 4 reales stamps cut horizontally in two, each half being used as 2 reales,

I57 $2 \mathrm{r}$ red on orange (half of 4 reales), upper half

I58 2r red on orange (half of 4 reales), lower half

c. 4 reales stamps cut diagonally in two, each half being used as 2 reales.

I59 $2 \mathrm{r}$ red on orange (half of 4 reales), upper right half

I60 $2 \mathrm{r}$ red on orange (half of 4 reales) lower right half

I6I 2r red on orange (half of 4 reales), upper left half

I62 2r red on orange (half of 4 reales), lower left half

d. 8 reales stamps cut vertically in two, each half being used as 4 reales.

I63 $4 \mathrm{r}$ green on brown (half of 8 reales), right half

I64 $4 \mathrm{r}$ green on brown (half of 8 reales), left half

e. 8 reales stamps cut horizontally in two, each half being used as 4 reales.

I65 4r green on brown (half of 8 reales), upper half

I66 4r green on brown (half of 8 reales), lower half

f. 8 reales stamps cut diagonally in two, each half being used as 4 reales.

I67 4 r green on brown (half of 8 reales), upper right half

I68 $4 \mathrm{r}$ green on brown (half of 8 reales), lower right half

I69 4r green on brown (half of 8 reales), upper left hal

I $704 \mathrm{r}$ green on brown (half of 8 reales), lower left half 

District Names found on THE Issue of I 86 I.

\begin{tabular}{|c|c|}
\hline District. & Meas, in mm. \\
\hline ACAPULCO. & I $81 / 2 \times 2$; bet. periods \\
\hline ACAPULCO & $18 \times 21 / 2$ \\
\hline AGUASCALTES & $21 \times 3$ \\
\hline ACUASCALIENTES & $23 \times 3$ \\
\hline 'APAM' & IO $1 / 2 \times 2$; bet. periods \\
\hline APAM & $9 \times 2$ \\
\hline $\begin{array}{l}\text { APAM } \\
\text { - CAMP ECHE. }\end{array}$ & $\begin{array}{l}81 / 2 \times 2 \\
19 \times 2 ; \text { bet. periods }\end{array}$ \\
\hline COLIMA & $123 / 4 \times 2$ \\
\hline CORDOVA. & $\begin{array}{l}\text { I } 6 \times 2 \text {; bet. periods } \\
\text { I } 6 \times 2\end{array}$ \\
\hline CUEIR N AVACA. & $\begin{array}{l}\text { I } 91 / 4 \times 1 / 4 ; \text { with one } \\
\text { period }\end{array}$ \\
\hline GUERNAVACA • & I9x2; with one period \\
\hline CHALCO & I $2 \times I 1 / 2$ \\
\hline C HIAPAS. & $\begin{array}{l}\text { I6x2; bet. periods, } \\
\text { sur'ge in blue }\end{array}$ \\
\hline -CHIHUAHUA. & $201 / 2 \times 2$; bet. periods \\
\hline CHIHUAHUA. & $\begin{array}{l}\text { I } 91 / 2 \times 11 / 20 \text {, with one } \\
\text { period }\end{array}$ \\
\hline -DURANGO' & I $81 / 2 \times$ I $1 / 2$; bet. periods \\
\hline -DURANGO. & I $7 \times 2$; bet. periods \\
\hline DURANGO & I $4 \times 2$ \\
\hline GUADALAJARA. & $20 \times 2$; with one period \\
\hline GUADALAJARA & $\begin{array}{l}201 / 2 \times 11 / 2 ; \text { without } \\
\text { period }\end{array}$ \\
\hline “، & $20^{I} / 2 \times 2 ;$ sur'ge in blue \\
\hline GUAD ALAJA RA & I $9 \times 2$ \\
\hline & I9x2; sur'ge in blue \\
\hline GUANAJUATO. & $23 \times 3$; with one period \\
\hline GUANAJUATO & $201 / 2 \times 13 / 4$ \\
\hline GUANAJUATO & $18 \times 13 / 4$ \\
\hline GUANAJUATO. & $\begin{array}{l}\text { I } 81 / 2 \times 1 \mathrm{~J} / 2 \text {; with one } \\
\text { period }\end{array}$ \\
\hline $\begin{array}{l}\text { HERHOSXILO- } \\
\text { HUEJUTLA }\end{array}$ & $\begin{array}{l}21 \times 2 \text {; bet. periods } \\
16 \times 2\end{array}$ \\
\hline -I. DEL CARMEN. & 2 Ix 2 ; bet. periods \\
\hline $\begin{array}{l}\text { IXTLAHUACA } \\
\text { J A I A PA. }\end{array}$ & $\begin{array}{l}\text { I } 9 \times 13 / 4 \\
153 / 4 \times 2 \text {; bet. periods }\end{array}$ \\
\hline -JALAPA. & I $41 / 2 \times 2$; bet. periods \\
\hline LAGOS & $\begin{array}{l}\text { II } \times 2 \mathrm{I} / 2 \\
\text { II } 1 / 2 \times 21 / 2\end{array}$ \\
\hline Lerma & (Caillebotte) \\
\hline MARAVATIO & I $7 \times 2$ \\
\hline MARAVATIO & I $6 \mathrm{XI} / 2$ to 3 \\
\hline $\begin{array}{l}\text { MAZATLAN } \\
\text { MAZATLAN } \\
\text { MERIDA }\end{array}$ & $\begin{array}{l}\text { I } 7 \times 2 \text {; bet. periods } \\
\text { I } 51 / 2 \times 13 / 4 \\
\text { I } 4 \times 2\end{array}$ \\
\hline MEXICO & $16 \times 23 / 4$ \\
\hline$M E X I C O$ & $14 \frac{1}{4} \times 21 / 4$ \\
\hline
\end{tabular}

\begin{tabular}{|c|c|}
\hline District. & Meas, in $\mathrm{mm}$. \\
\hline MI ISXICCO & $14 \times 21 / 2$ \\
\hline M EXI CO & $14 \times 2 \mathrm{I} / 4$ \\
\hline MEXICO & $13 \mathrm{I} / 2 \times 2 \mathrm{I} / 4$ \\
\hline MEXICo & $131 / 2 \times 21 / 2$ to $I 3 / 4$ \\
\hline $\mathbb{M} \mathbb{X} \times \mathbb{C} \mathbb{D}$ & $16 \times 2$ \\
\hline -MONTERREY & $20 \times 13 / 4$; bet. periods \\
\hline MORELIA & $131 / 2 \times x 2$ \\
\hline MORELIA & $14 \times 2$ \\
\hline MORELIA. & $\begin{array}{l}\text { I } 6 \times 11 / 2 ; \text { with one } \\
\text { period }\end{array}$ \\
\hline - OAJACA. & I $5 \times 2$; bet. periods \\
\hline O R I ZAVA. & $163 / 4 \times 2$ \\
\hline OORIZAVA. & I $51 / 2 \times 11 / 2 ;$ bet. periods \\
\hline PACHUCA & $13 \times I 1 / 2$ \\
\hline & $24 \times 43 / 4$ \\
\hline PUEBLA & $19 \times 3$ \\
\hline PUEBLA & $12 \times 23 / 4$ \\
\hline PUEBLA. & $\begin{array}{l}\text { I3 } 3 / 4 \times 23 / 4 ; \text { with one } \\
\text { period }\end{array}$ \\
\hline QUERE TARO & I $71 / 2 \times 13 / 4$ \\
\hline QUERETARO & $17 \times 2$ to $21 / 4$ \\
\hline QQUERETARO' & I $71 / 2 \times 13 / 4$; bet. periods \\
\hline & $\begin{array}{l}261 / 2 \times 31 / 4 ; \text { with one } \\
\text { period }\end{array}$ \\
\hline
\end{tabular}

$\mathbf{S} \cdot \mathbf{L} \cdot \mathbf{P O T O S I} \cdot \quad 23^{1 / 2} \times 3$; with one period S. L. POTOSI $\quad 22 \times 2 \frac{3}{4}$; with one period

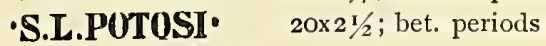
S. L.POTOSI I $8 \times 2$ to 4 S. L.POTOSI $\quad \mathrm{7} 7 \times 2$ SOYANIQUILPAM $20 \times 2$ Sultepec (Caillebotte) -T A B A SCO. I6xi $1 / 2$; bet. periods -TAMPIC0- I6 0 ; ; bet. periods TAMPICO $I 4 \times 2$ TIXTLA I $4 \times 2$ -TLALPUJAHUA. 22xI $3 / 4$; bet. periods TOL UCA I $2 \times 1 / 4$ TULA $9 \times 1 / 2$ TULA NCI NCO I9XI $3 / 4$ 'VERACRUZ• $\quad$ I $7 \frac{1}{2} \times 2$; bet. periods VERACRUZ• I $18 \times 2$;(ornamental type) VERA CRUZ I6 2 VERA CRUZ. I $5 \times 13 / 4$; with one period . VICT' ${ }^{A}$ TAMPAS $241 / 2 \times 21 / 2$; bet. periods ZACATECAS $201 / 2 \times 2 \frac{1}{2}$ Zacatecas in MS.; (Caillebotte)

ERRORS.

Names of postal districts found surcharged twice on stamps of this issue.

\begin{tabular}{|c|c|c|c|c|c|}
\hline $\begin{array}{l}\text { District. } \\
\text { Morelia }\end{array}$ & $\begin{array}{l}\text { Value. } \\
2 r\end{array}$ & $\begin{array}{l}\text { Color. } \\
\text { black on pink }\end{array}$ & $\begin{array}{l}\text { Meas. of Sur. } \\
\text { I6xI } 1 / 2\end{array}$ & $\begin{array}{l}\text { Color. } \\
\text { black }\end{array}$ & surcharged twice \\
\hline Vera Cruz & Ir & black on green & $171 / 2 \times 2$ & “" & \\
\hline
\end{tabular}


MEXICO.

End of 1863 .

Head of Hidalgo engraved on white and yellowish white wove paper. Size, $20 \times 25$ $\mathrm{mm}$.

Perforated I2.
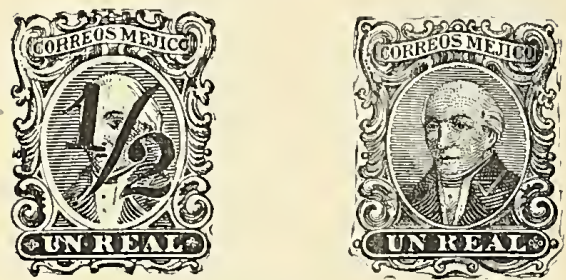

I. Yellowish white wove paper.

$\mathrm{I}^{\circ}$ Without name of postal district.

I (un) $r$ red

2 (dos) r blue

4 (cuatro) $r$ brown

I (un) p black

$1 / 2 \mathrm{r}$ on $\mathrm{Ir}$ red

$2^{\circ}$ With name of postal district

I (un) $r$ red

2 (dos) $r$ blue

4 (cuatro) $r$ brown

I (un) p black

$\mathrm{r} / 2 \mathrm{r}$ on Ir red

II. White wove paper.

Without name of postal district.

Perforated I2.

I80 I (un) $r$ red

I8I 2 (dos) $r$ blue

I 824 (cuatro) $r$ stone brown

I83 I (un) p black

District NAMES FOUND ON I863 ISSUE. District.

CHIHUAHUA Meas, in $\mathrm{mm}$.

SALTILLO I5XI $1 / 2$

:SALTILLO- $\quad 17 \times 2 \frac{1}{4} ; 2$ periods on the $1 / 2$ real.

PASO DEL NORTE

(?)

I 864 .

Engraved on various papers. Size $191 / 2 x$ $25 \mathrm{~mm}$.

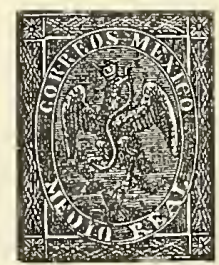

I. Without surcharge.

$I^{\circ}$ Vertically ribbed paper.

I84 3c brown
I $85 \quad 1 / 2$ (Medio) $r$ brown

I $86 \mathrm{I} / 2$ (Medio) r lilac

$187 \quad 1 / 2$ (Medio) r lilac brown

I88 $1 / 2$ (Medio) $\mathrm{r}$ red lilac

I $89 \quad 1 / 2$ (Medio) $r$ gray

I90 I (un) r gray blue

I9I I (un) r ultramarine

192 I (un) $r$ pale blue

I93 I (un) $r$ Prussian blue

I94 I (un) $r$ dark blue

I95 I (un) $r$ green blue

Ig6 2 (dos) $r$ yellow
I97 2 (dos) $r$ red orange

Ig8 2 (dos) $r$ orange

I99 4 (cuatro) $r$ green

2004 (cuatro) $r$ pale green

2014 (cuatro) $r$ dark green

2024 (cuatro) $\mathrm{r}$ yellow green

2034 (cuatro) $r$ bluish green

2048 (ocho) r red

2058 (ocho) r pale red

2068 (ocho) $r$ brick red

$2^{\circ}$ Thin wove paper.

$3 \mathrm{c}$ brown

$1 / 2$ (Medio) $r$ brown

$1 / 2$ (Medio) $r$ gray

$1 / 2$ (Medio) $r$ lilac

$1 / 2$ (Medio) $\mathrm{r}$ lilac brown

$1 / 2$ (Medio) $r$ red lilac

I (un) $r$ gray blue

I (un) $r$ ultramarine

I (un) $r$ pale blue

I (un) r Prussian blue

I (un) $r$ dark blue

I (un) $r$ green blue

2 (dos) r yellow

2 (dos) $r$ red orange

2 (dos) $r$ orange

4 (ctiatro) $r$ green

4 (cuatro) r pale green

4 (cuatro) r dark green

4 (cuatro) r yellow green

4 (cuatro) $r$ bluish green

8 (ocho) r red

8 (ocho) r pale red

8 (ocho) r brick red

$3^{\circ}$ Laid paper.

$3 \mathrm{c}$ brown

$1 / 2$ (Medio) $r$ brown

1/2 (Medio) $r$ gray

$1 / 2$ (Medio) $r$ lilac

$1 / 2$ (Medio) r lilac brown

$1 / 2$ (Medio) $r$ red lilac

I (un) r gray blue

I (un) $r$ ultramarine

I (un) $r$ pale blue

I (un) r Prussian blue

I (un) $r$ dark blue

$\mathrm{I}$ (un) $\mathrm{r}$ green blue

2 (dos) $r$ yellow

2 (dos) $r$ red orange

2 (dos) $r$ orange

4 (cuatro) $r$ green

4 (cuatro) $r$ pale green

4 (cuatro) $r$ dark green

4 (cuatro) $r$ bluish green

8 (ocho) r red

8 (ocho) $r$ pale red

8 (ocho) $r$ brick red

The stamps on laid paper are far from being as scarce as generally supposed, in fact they are quite common, but it is a rather difficult to see the vergures, it being necessary to hold them at a certain angle to the light in order to see the vergures on the back or face of the stamp.

$4^{\circ}$ Ordinary wove paper.

3c brown

$1 / 2$ (Medio) $r$ brown

$1 / 2$ (Medio) $r$ gray

$1 / 2$ (Medio) $\mathrm{r}$ lilac

$1 / 2$ (Medio) $r$ lilac brown

$1 / 2$ (Medio) $\mathrm{r}$ red lilac

I (un) $r$ gray blue

I (un) $r$ gray blue

I (un) $r$ ultramarine

I (un) $r$ pale blue

I (un) r Prussian blue

I (un) $r$ dark blue 




\begin{tabular}{|c|c|}
\hline 264 & I (un) r green blue \\
\hline 265 & 2 (dos) r yellow \\
\hline 266 & 2 (dos) $r$ orange red \\
\hline 267 & 2 (dos) $r$ orange \\
\hline 268 & 4 (cuatro) r green \\
\hline 269 & 4 (cuatro) r pale green \\
\hline 270 & 4 (cuatro) r dark green \\
\hline 271 & 4 (cuatro) r yellow green \\
\hline 272 & 4 (cuatro) r bluish green \\
\hline 273 & 8 (ocho) r red \\
\hline 274 & 8 (ocho) r pale red \\
\hline 275 & 8 (ocho) r brick red \\
\hline & eties: \\
\hline a. & rror of color. \\
\hline 276 & 8 (ocho) r Prussian blue \\
\hline b. & $\begin{array}{l}\text { reales stamps cut vertically in two, } \\
\text { lf being used as I real. }\end{array}$ \\
\hline 277 & Ir orange (half of 2 reales), right half \\
\hline 278 & Ir orange (half of 2 reales), left half \\
\hline c. & $\begin{array}{l}\text { reales stamps cut horizontally in two, } \\
\text { If being used as I real. }\end{array}$ \\
\hline 279 & Ir orange (half of 2 reales), upper half \\
\hline 280 & Ir orange (half of 2 reales), lower half \\
\hline $\begin{aligned} & d- \\
& \text { each }\end{aligned}$ & $\begin{array}{l}\text { reales stamps cut diagonally in two, } \\
\text { lf being used as I real. }\end{array}$ \\
\hline $28 \mathrm{I}$ & $\begin{array}{l}\text { Ir orange (half of } 2 \text { reales), upper right } \\
\text { half }\end{array}$ \\
\hline 282 & $\begin{array}{l}\text { Ir orange (half of } 2 \text { reales), lower right } \\
\text { half }\end{array}$ \\
\hline 283 & $\begin{array}{l}\text { Ir orange (half of } 2 \text { reales) upper left } \\
\text { half }\end{array}$ \\
\hline 284 & $\begin{array}{l}\text { Ir orange (half of } 2 \text { reales), lower left } \\
\text { half }\end{array}$ \\
\hline
\end{tabular}

e. 4 reales stamps cut vertically in two, each half being used as 2 reales.

$285 \quad 2$ r green (half of 4 reales), right half 286 2r green (half of 4 reales), left half

f. 4 reales stamps cut horizontally in two, each half being used as 2 reales.

$287 \quad 2$ r green (half of 4 reales), upper half 2882 r green (half of 4 reales), lower half

g. 4 reales stamps cut diagonally in two, each half being used as 2 reales.

2892 2r green (half of 4 reales), upper right half

$290 \quad 2 \mathrm{r}$ green (half of 4 reales), lower right half

29 I 2 r green (half of 4 reales), upper left half

292 2r green (half of 4 reales), lower left half

h. 8 reales stamps cut vertically in two, each half being used as 4 reales.

$2934 \mathrm{r}$ red (half of 8 reales), right half

2944 r red (half of 8 reales), left half

i. 8 reales stamps cut horizontally in two, each half being used as 4 reales.

$2954 \mathrm{r}$ red (half of 8 reales), upper half

2964 r red (half of 8 reales), lower half

j. 8 reales stamps cut diagonally in two, each half being used as 4 reales.

2974 r red (half of 8 reales), upper right half

$2984 \mathrm{r}$ red (half of 8 reales), lower right half

$2994 \mathrm{r}$ red (half of 8 reales), upper left half

$3004 \mathrm{r}$ red (half of 8 reales), lower left half

II. Surcharged with name of town only.

$I^{\circ}$ Vertically ribbed paper.

3OI $3 \mathrm{c}$ brown

$302 \quad \mathrm{r} / 2$ (Medio) $r$ brown

$303 \quad 1 / 2$ (Medio) $\mathbf{r}$ lilac

$304 \quad 1 / 2$ (Medio) $r$ red Iilac

305 I (un) $r$ gray blue
306 I (un) r ultramarine

307 I (un) r Prussian blue (I865)

3082 (dos) $r$ yellow

3092 (dos) $r$ orange

3 IO 2 (dos) $r$ red orange

3 II 4 (cuatro) r green

3 I2 4 (cuatro) r yellow green

3I3 8 (ocho) r red

3148 (ocho) r brick red

$2^{\circ}$ Thin wove paper.

\section{I5 3c brown}

$3 I 6 \quad 1 / 2$ (Medio) r brown

3 I $1 / 2$ (Medio) $r$ lilac

$3 I 8 \quad 1 / 2$ (Medio) r red lilac

3I9 I (un) r gray blue

320 I (un) r ultramarine

32 I (un) r Prussian blue

3222 (dos) $r$ yellow

3232 (dos) $r$ orange

3242 (dos) $r$ red orange

3254 (cuatro) $r$ green

3264 (cuatro) $r$ yellow green

3278 (ocho) r red

3288 (ocho) r brick red

$3^{\circ}$ Laid paper.

$3293 \mathrm{c}$ brown

$330 \quad 1 / 2$ (Medio) $r$ brown

33 I $1 / 2$ (Medio) r lilac

$332 \quad 1 / 2$ (Medio) $r$ red lilac

333 I (un) r gray blue

334 I (un) r ultramarine

335 I (un) r Prussian blue

3362 (dos) r yellow

3372 (dos) r orange

3382 (dos) $r$ red orange

3394 (cuatro) r green

3404 (cuatro) r yellow green

$34 \mathrm{I} \quad 8$ (ocho) r red

3428 (ocho) $r$ brick red

$4^{\circ}$ Ordinary laid paper.

$343 \quad 3$ c brown

$344 \quad \mathrm{r} / 2$ (Medio) $\mathrm{r}$ brown

$345 \quad 1 / 2$ (Medio) $r$ lilac

$346 \quad 1 / 2$ (Medio) $r$ red lilac

347 I (un) gray blue

348 I (un) $r$ ultramarine

349 I (un) r Prussian blue

3502 (dos) $r$ yellow

$35 \mathrm{I} 2(\mathrm{dos}) \mathrm{r}$ orange

3522 (dos) r red orange

3534 (cuatro) $r$ green

3544 (cuatro) r yellow green

3558 (ocho) r red

3568 (ocho) $r$ brick red

Varieties :

a. 2 reales stamps cut vertically in two, each half been used as I real.

$356 \mathrm{a}$ Ir orange (half of 2 reales), right half

357 Ir orange (half of 2 reales), left half.

b. 2 reales stamps cut horizontally in two, each half being used as I real.

$357 \mathrm{a}$ Ir orange (half of 2 reales), upper half

$35^{8} 2$ r orange (half of 2 reales), lower half

c. 2 reales stamps cut diagonally in two, each half being used as I real.

$358 \mathrm{a}$ ir orange (half of 2 reales), upper right half

359 Ir orange (half of 2 reales), lower right half

360 Ir orange (half of 2 reales), upper left half

$360 a$ Ir orange (half of 2 reales), lower left half 
d. 4 reales stamps cut vertically in two, each half being used as 2 reales.

$36 \mathrm{I} 2 \mathrm{r}$ green (half of 4 reales), right half 362 2r green (half of 2 reales), left half

e. 4 reales stamps cut horizontally in two, each half being used as 2 reales.

363 2r green (half of 4 reales), upper half 364 2r green (half of 4 reales), lower half

f. 4 reales stamps cut diagonally in two, each half being used as 2 reales.

365 2r green (half of 4 reales), upper right half

$3662 \mathrm{r}$ green (half of 4 reales), lower right half

$3672 \mathrm{2}$ green (half of 4 reales), upper

$3682 \mathrm{2r}$ green (half of 4 reales), lower left half g. 8 reales stamps cut vertically in two, each half being used as 4 reales.

$3694 \mathrm{r}$ red (half of 8 reales), right half

$370 \quad 4 \mathrm{r}$ red (half of 8 reales), left half

h. 8 reales stamps cut horizontally in two, each half being used as 4 reales.

$37 \mathrm{I} \quad 4 \mathrm{r}$ red (half of 8 reales), upper half

$3724 \mathrm{r}$ red (half of 8 reales), lower half

i. 8 reales stamps cut diagonally in two, each half being used as 4 reales.

$3734 \mathrm{r}$ red (half of 8 reales), upper right half

$3744 \mathrm{r}$ red (half of 8 reales), lower right half

$3754 \mathrm{r}$ red (half of 8 reales), upper left half.

$376 \quad 4 \mathrm{r}$ red (half of 8 reales), lower left half

DISTRICT NAMES FOUND ON THIS Issue.

\begin{tabular}{|c|c|}
\hline District. & Meas. in nım. \\
\hline AGUASCALTES & $23 \times 3$ \\
\hline AGUASCALTES. & $22 \times 3$; with period \\
\hline APAM & $91 / 2 \times 2$ \\
\hline BARCA & $9 \times 21 / 4$ \\
\hline COCULA & $12 \times 2$ \\
\hline - CORDOVA. & I $6 \times 2$; bet. periods \\
\hline CUERNAVACA. & I $8 \times 2 ;$ with period \\
\hline CHALCO & I $3 \times 13 / 4$ \\
\hline GUADALAJARA & $201 / 2 \times 21 / 2$ \\
\hline GUADALAJARA & $20 \times 2$ \\
\hline GUANAJUATO. & $23 \times 3$; with period \\
\hline HIDALGO & I $3 \times 2$ \\
\hline JALAPA & $\begin{array}{l}\text { I01/2x2; with one } \\
\text { period }\end{array}$ \\
\hline JALAPA• & I $4 \times 2$; with one period \\
\hline LAGOS & $\operatorname{I0x} 2$ \\
\hline LERMA & $10 \times 2$ \\
\hline MERIDA & I $3 \times 2$ \\
\hline
\end{tabular}

\begin{tabular}{|c|c|}
\hline $\begin{array}{c}\text { District. } \\
\text { Soexico }\end{array}$ & $\begin{array}{l}\text { Meas. in mm. } \\
\text { I } 5 \times 2\end{array}$ \\
\hline IVorelia & $161 / 2 \times 13 / 4$ \\
\hline 'ORIZAVA' & I $5 \times 13 / 4$ \\
\hline PACHUCA & $13 \times 2$ \\
\hline PUEBLA. & $131 / 2 \times 21 / 3$; with perio \\
\hline QUERETARO & I $7 \times 1 / 8 ;$ with one perio \\
\hline S.L.POTOSI & $161 / 2 \times 2$ \\
\hline & $153 / 4 \times 2$ \\
\hline TAMPICO & $131 / 2 \times 13 / 4$ \\
\hline TEPIC & $71 / 4 \times 2$ \\
\hline TEPE J I & $\operatorname{IIX} 2$ \\
\hline TOLUCA & $141 / 2 \times 2$ \\
\hline TULANCINGO & $18 \times 21 / 4$ \\
\hline -VERACRUZ. & I $8 \times 21 / 2 ;$ bet. periods \\
\hline 'VERA CRUZ' & I6 $6 / 2 \times 2$; bet. periods \\
\hline YGUALA & $13 \times 2$ \\
\hline
\end{tabular}

ERRORS

Name of postal district found surcharged twice.

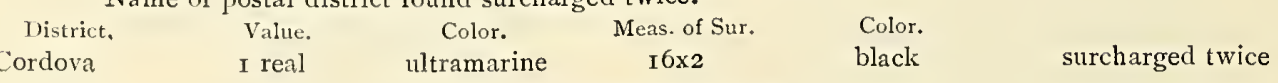

HABILITADOS.

Mexico and Yguala on 2 reales yellow.

Aguascaltes and Zacatecas on 2 reales yellow

III. Surcharged with name of town, and

number and date in large Egyptian type.

$I^{\circ}$ Vertically ribbed paper.

$3 \mathrm{c}$ brown

$3 \mathrm{c}$ red brown

$x / 2$ (Medio) $r$ brown

$1 / 2$ (Medio) $r$ lilac brown

$1 / 2$ (Medio) $\mathrm{r}$ red lilac

I (un) $r$ blue gray

I (un) $\mathrm{r}$ ultramarine

2 (dos) $r$ yellow

2 (dos) $r$ orange

2 (dos) $r$ red orange

4 (cuatro) r green

4 (cuatro) $r$ yellow green

8 (ocho) $r$ red

8 (ocho) $r$ pale red

$2^{\circ}$ Laid paper.

$3 \mathrm{c}$ brown

$3 \mathrm{c}$ red brown

$1 / 2$ (Medio) $r$ brown

$1 / 2$ (Medio) $r$ lilac brown

$1 / 2$ (Medio) $\mathrm{r}$ red lilac

I (un) $r$ blue gray

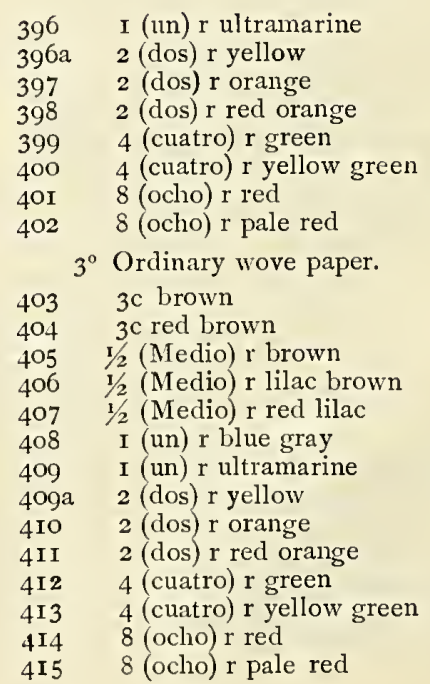



Varieties:

a. 2 reales stamps cut vertically in two, each half being used as I real.

4I6 Ir orange (half of 2 reales), right half

$4 \mathrm{I} 7$ Ir orange (half of 2 reales), left half

b. 2 reales stamps cut horizontally in two, each half being used as I real.

4 I 8 Ir orange (half of 2 reales), upper half

4 I9 2 r orange (half of 2 reales), lower half

c. 2 reales stamps cut diagonally in two, each half being used as I real.

420 Ir orange (half of 2 reales), upper right half

42I Ir orange (half of 2 reales), lower right half

422 Ir orange (half of 2 reales), upper left half 423 Ir orange (half of 2 reales), lower left

d. 4 reales stamps cut vertically in two, each half being used as 2 reales.

$424 \quad 2$ green (half of 4 reales), right half

4252 2r green (half of 2 reales), left half

e. 4 reales stamps cut horizontally in two, each half being used as 2 reales.

$426 \quad 2 r$ green (half of 4 reales), upper half

$427 \quad 2 \mathrm{r}$ green (half of 4 reales), lower half

f. 4 reales stamps cut diagonally in two, each half being used as 2 reales.

4282 2r green (half of 4 reales), upper right half

4292 2r green (half of 4 reales), lower right half

430

2r green (half of 4 reales), upper left half

2 green (half of 4 reales), lower left half

g. 8 reales stamps cut vertically in two, each half being used as 4 reales.

$4324 \mathrm{r}$ red (half of 8 reales), right half

$4334 \mathrm{r}$ red (half of 8 reales), left half

h. 8 reales stamps cut horizontally in two, each half being used as 4 reales.

$434 \quad 4 \mathrm{r}$ red (half of 8 reales), upper half

$4354 \mathrm{r}$ red (half of a reales), lower half

i. 8 reales stamps cut diagonally in two, each half being used as 4 reales.

$436 \quad 4 \mathrm{r}$ red (half of 8 reales), upper right half

$4 \mathrm{r}$ red (half of 8 reales), lower right half

$4384 \mathrm{r}$ red (half of 8 reales), upper left half $4 \mathrm{r}$ red (half of 8 reales), lower left
half

IV. Surcharged with number and date only.

I $^{\circ}$ Vertically ribbed paper.

$3 \mathrm{c}$ red brown

$1 / 2$ (Medio) $r$ brown

$1 / 2$ (Medio) r lilac brown

$1 / 2$ (Medio) $r$ red lilac

I (un) $r$ blue gray

I (un) $r$ ultramarine

2 (dos) $r$ orange

2 (dos) $r$ red orange

4 (cuatro) $r$ green

4 (cuatro) r pale green

8 (ocho) $r$ red

8 (ocho) r pale red

$2^{\circ}$ Laid paper.

$3 \mathrm{c}$ brown

$3 \mathrm{c}$ red brown

$1 / 2$ (Medio) $r$ brown

$r / 2$ (Medio) $r$ lilac brown

$1 / 2$ (Medio) r red lilac
458 I (un) I blue gray.

459 I (un) r ultramarine

4602 (dos) $r$ orange

4612 (dos) $r$ red orange

4624 (cuatro) r green

4634 (cuatro) r pale green

4648 (ocho) r red

4658 (ocho) $r$ pale red

$3^{\circ}$ Ordinary wove paper

466 3c brown

$467 \quad 3 \mathrm{c}$ red brown

$468 \quad 1 / 2$ (Medio) r brown

$469 \quad 1 / 2$ (Medio) r lilac brown

$470 \quad 1 / 2$ (Medio) $r$ red lilac

47I I (un) r blue gray

472 I (un) $r$ ultramarine

4732 (dos) $r$ orange

4742 (dos) $r$ red orange

4754 (cuatro) r green

4764 (cuatro) $r$ pale green

4778 (ocho) r red

$478 \quad 8$ (ocho) r pale red

Varieties:

a. 2 reales stamps cut vertically in two, each half being used as $I$ real.

479 Ir orange (half of 2 reales), right half

480 Ir orange (half of 2 reales), left half

b. 2 reales stamps cut horizontally in two, each half being used as I real.

$48 \mathrm{I}$ Ir orange (half of 2 seales), upper half

482 Ir orange (half of 2 reales), lower half

c. 2 reales stamps cut diagonally in two, each half being used as $\mathbf{I}$ real.

483 Ir orange (half of 2 reales), upper right half

484 Ir orange (half of 2 reales), lower right half

485 Ir orange (half of 2 reales), upper left half

486 Ir orange (half of 2 reales), lower left half

d. 4 reales stamps cut vertically in two, each half being used as 2 reales.

487 2r green (half of 4 reales), right half

4882 2r green (half of 4 reales), left half

e. 4 reales stamps cut horizontally in two, each half being used as 2 reales.

$489 \quad 2 r$ green (half of 4 reales), upper half $490 \quad 2 r$ green (half of 4 reales), lower half

f. 4 reales stamps cut diagonally in two, each half being used as two reales.

49 I 2r green (half of 4 reales), upper right half

$492 \quad 2$ green (half of 4 reales), lower right half 493 2r green (half of 4 reales), upper left

494

2r green (half of 4 reales), lower left half

g. 8 reales stamps cut vertically in two, each half being used as 4 reales.

$4954 \mathrm{r}$ red (half of 8 reales), right half

$496 \quad 4 \mathrm{r}$ red (half of 8 reales), left half

h. 8 reales stamps cut horizontally in two, each half being used as 4 reales.

$+974 \mathrm{r}$ red (half of 8 reales), upper half

$4984 \mathrm{r}$ red (half of 8 reales), lower half

i. 8 reales stamps cut diagonally in two, each half being used as 4 reales.

$4994 \mathrm{r}$ red (half of 8 reales), upper right half

$500 \quad 4 \mathrm{r}$ red (half of 8 reales), lower right half

5OT

$4 \mathrm{r}$ red (half of 8 reales), upper left half

502 
District Names of i864 Issue, with Number and Date in Large Egyptian Type.

\begin{tabular}{|c|c|c|c|}
\hline $\begin{array}{l}\text { District. } \\
\text { AGUASCALTES. }\end{array}$ & $\begin{array}{l}\text { Meas, in mm. } \\
22 \times 3 \text {; with period }\end{array}$ & $\begin{array}{l}\text { District. } \\
\text { PIERTA. }\end{array}$ & $\begin{array}{l}\text { Meas. in } \mathrm{mm} \text {. } \\
\mathrm{I} 31 / 2 \times 2 \mathrm{x} / 3 \text {; with period }\end{array}$ \\
\hline CORDOVA. & I $6 \times 2$; bet. periods & QUERETARO & $17 \times 17 / 8$ \\
\hline CUAUTITLAN & $17 \times 2$ & QUERETARO & I $71 / 2 \times 3$ to $I x / 2$ \\
\hline CUERNAVACA & $18 \times 2$ & S.L.POTOSI & $161 / 2 \times 2$ \\
\hline CHALCO & $\mathrm{I} 3 \times \mathrm{I} 3 / 4$ & SOYANIQUILPAM & $20 \times 2$ \\
\hline $\begin{array}{l}\text { DURANG0 } \\
\text { GUADA LAJARA }\end{array}$ & $\begin{array}{l}15 \times 2 \\
20 \times 2\end{array}$ & TAMPICO & $131 / 2 \times 2$ \\
\hline GUANAJUATO. & $23 \times 3$; with period & TEP E J I & I Ix 2 \\
\hline I DEL GARMEN. & $20 \times 2$; with period & TEPIC & $71 / 2 \times 2$ \\
\hline IXTLA IIUACA & $18 \times 2$ & TOLUCA & $\begin{array}{l}22 \times 2 \\
14 \times 2\end{array}$ \\
\hline $\begin{array}{l}\text { JALAPA } \\
\text { LA GOS }\end{array}$ & $101 / 2 \times 2$ & TELA & $\begin{array}{l}14 \times 2 \\
8 \times 2\end{array}$ \\
\hline $\begin{array}{l}\text { Latos } \\
\operatorname{arexica}\end{array}$ & $\begin{array}{l}10 \times 2 \\
15 \times 2\end{array}$ & TULANCINGO & $18 \times 21 / 4$ \\
\hline MONTERREY & $\operatorname{Ig} \times 1 / 2$ & TULANCINGO. & I9x2; with period \\
\hline $\begin{array}{l}\text { MTorelia } \\
\text { ORIZAVA. }\end{array}$ & $\begin{array}{l}161 / 4 \times 13 / 4 \\
15 \times 13 / 4 \text {; bet. periods }\end{array}$ & $\begin{array}{l}\text {-VERACRUZ• } \\
\text { VERA CRUZ }\end{array}$ & $\begin{array}{l}\text { I } 7 \times 2 ; \text { bet. periods } \\
\text { I } 6 \times 2 ; \text { with period }\end{array}$ \\
\hline PACHUCA & $13 \times 2$ & ZACATECAS & $25 \times 3$; with period \\
\hline
\end{tabular}

The following numbers show where each consignment was sent, the numbers running from II 8 to I 79 :

$\begin{array}{ll}\text { II8 } & \text { Guanajuato } \\ \text { II8 } & \text { Mexico } \\ \text { II9 } & \text { Mexico } \\ \text { I20 } & \text { Puebla } \\ \text { I21 } & \text { Cuernavaca } \\ \text { I22 } & \text { Jalapa } \\ \text { I22 } & \text { Mexico } \\ \text { I23 } & \text { Mexico } \\ \text { I24 } & \text { Soyaniquilpam } \\ \text { I25 } & \text { Puebla } \\ \text { I26 } & \text { Orizava } \\ \text { I27 } & \text { S. L. Potosi } \\ \text { I28 } & \text { Vera Cruz } \\ \text { I29 } & \text { Queretaro } \\ \text { I30 } & \text { Mexico } \\ \text { I31 } & \text { Puebla } \\ \text { I32 } & \text { Jalapa } \\ \text { I33 } & \text { Cordova }\end{array}$

$\begin{array}{ll}\text { I34 } & \text { Tlalpujahua } \\ \text { I35 } & \text { Puebla } \\ \text { I36 } & \text { Pachuca } \\ \text { I37 } & \text { Chalco } \\ \text { I38 } & \text { Mexico } \\ \text { I39 } & \text { Cuautitlan } \\ \text { I40 } & \text { ? } \\ \text { I4I } & \text { Morelia } \\ \text { I42 } & \text { Mexico } \\ \text { I43 } & \text { Tepeji } \\ \text { I44 } & \text { Mexico } \\ \text { I45 } & \text { Tampico } \\ \text { I46 } & \text { Guadalajara } \\ \text { I47 } & \text { Puebla } \\ \text { I48 } & \text { Guanajuato } \\ \text { I49 } & \text { Mexico } \\ \text { I50 } & \text { ? } \\ \text { I5I } & \text { Pachuca }\end{array}$

$\begin{array}{ll}\text { I52 } & \text { Vera Cruz } \\ \text { I53 } & \text { Mexico } \\ \text { I54 } & \text { Mexico } \\ \text { I54 } & \text { S. L. Potosi } \\ \text { I55 } & \text { S. L. Potosi } \\ \text { I56 } & \text { Durango } \\ \text { I56 } & \text { S. L. Potosi } \\ \text { I57 } & \text { Toluca } \\ \text { I57 } & \text { Chalco } \\ \text { I58 } & \text { ? } \\ \text { I59 } & \text { Orizava } \\ \text { I59 } & \text { Cordova } \\ \text { I60 } & \text { Tulancingo } \\ \text { I6 I } & \text { Cuernavaca } \\ \text { I62 } & \text { Vera Cruz } \\ \text { I63 } & \text { Mexico } \\ \text { I64 } & \text { I del Carmen } \\ \text { I65 } & \text { Mexico }\end{array}$

$\begin{array}{ll}\text { I66 } & \text { Zacatecas } \\ \text { I66 } & \text { Aguascaltes } \\ \text { I67 } & \text { Guadalajara } \\ \text { I68 } & \text { Ixtlahuaca } \\ \text { I69 } & \text { ? } \\ \text { I70 } & \text { Puebla } \\ \text { I70 } & \text { S. L. Potosi } \\ \text { I7I } & \text { Queretaro } \\ \text { I72 } & \text { Lagos } \\ \text { I73 } & \text { Mexico } \\ \text { I74 } & \text { Pachuca } \\ \text { I75 } & \text { Monterey } \\ \text { I76 } & \text { Guanajuato } \\ \text { I77 } & \text { Mexico } \\ \text { I78 } & \text { Queretaro } \\ \text { I78 } & \text { Tula } \\ \text { I79 } & \text { Puebla }\end{array}$

SURChARges of Sub-Districts.

It has long been a puzzle to advanced collectors of the stamps of Mexico, as to the meaning of the single numbers found (in addition to the regular surcharge of control number and date), surcharged on the "Eagle" issues of I864-66, and the "Maximilian" issues of I866-67. Mr. Albert E. Lawrence thinks he has solved the mystery, the fact being that they have the same significance as the stamps of the-e issues found surcharged with two numbers and dates, and which have been commonly called in the past "Habilitados" This term is however, erroneous, as the second surcharged series of numbers and dates, or of numbers alone, were simply a system of control numbers used by the various postal districts in sending consignments to the various sub-stations situated within their precincts. The boundaries of the postal districts apparently were not always confined to state lines, as will be shown in some of the following tables: For instance Zacatecas, which furnished most of the stamps used in Aguascaltes; S. L. Potosi, from whence many consignments were sent to Tula de Tamaulipas; etc., etc.

Some of the series are of far greater interest than the others, as for instance Guadalajara, where the sub-stations surcharged their names on the stamps which were sent to them without surcharge of the name of the issuing district. This is al:o the case with the consignments sent from Zacatecas to Aguascallienles, which are almost always found surcharged only Aguascalıes. Generally, however, the only method of ascertaining the destination of the consignments is by a careful study of the postmarks, and it is surpri-ing how expert one can become in a short time in deciphering them. This is the case where the name of the town is given as part of the cancellation, which is almo-t always done, but when it merely consists of some fancy creation, such as are found in the series of Guanajuato, they can only be placed by finding the stamps on the entire letiers. It will be remembered that the Central Office of Mexico did not commence surcharging the numbers on the consignments sent to the various postal districts unil some months after the appearance of the "Eagle" issue, and then the first number found is II $8-1864$, showing that II 7 consignments had been sent out before the system of numbering was begun. This the writer believes was also the case with the consignments sent from some of the postal districts to their sub-stations; for example, the earliest number he 
• 

has found of the series of Queretaro, in 1864 is $4 \mathrm{I}$, and of the series of Puebla, in 1864 , 44; careful search through large lots of these stamps failing to unearth any lower numbers. It will probably take years of patient examination to attain a complete knowledge of these interesting series. The writer considers that he has made a beginning and hopes that others will be found to assist in completing the tables which follow.
The greatest difficulty will be found in tracing the "Maximilian" series, the stamps being so much rarer than the "Eagle" design. After the extinction of the Empire, several of the sub-districts were considered of sufficient importance to have consignments sent them direct from Mexico; for instance Cocula, Tepic, Tehuacan and Zamora in 1874 , and Tula de Tamaulipas in $\mathbf{1} 868$.

Series of Guadalajara (State of Jalisco)

This series differs from all others in that the numbers were commenced sometime in 1864 and contınued consecutively through $\mathrm{I} 865$ beginning again with number I, in I866. Also in that the first I5 numbers were also surcharged with the date as well. Number I5 $^{-1865}$ was surcharged Zapotlan, and number 15 without date appears to have been used both in Tepic \& Colima; with the exception of this number all known to the writer seem to have been used only in the sub-districts designated in the following table. Where the town name is in brackets it means that it is not surcliarged on the stamp, and is only known by the cancellation.

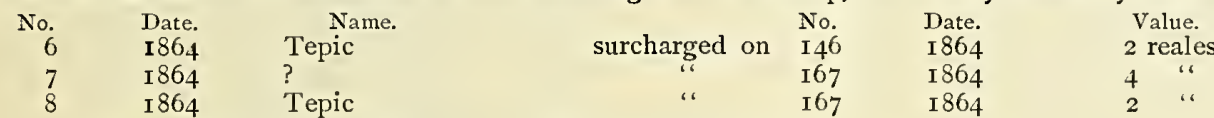

Series of S. L. Potosi (State of San Luis Potosi).

This series is surcharged with the extra number only, and also only bears the name of the issuing district-S. L. Potosi. They are very numerous and the system of numbering the consignments began in 1864 .

\begin{tabular}{|c|c|c|c|c|c|c|}
\hline No. & $\begin{array}{l}\text { Name. } \\
\text { Matehuala }\end{array}$ & surcharged on & $\begin{array}{l}\text { No. } \\
127\end{array}$ & $\begin{array}{l}\text { Date. } \\
\text { I } 864\end{array}$ & \multicolumn{2}{|c|}{$\begin{array}{l}\text { Value. } \\
2 \text { reales }\end{array}$} \\
\hline 4 & Salinas & " & I 55 & I 864 & 2 & "6 \\
\hline 6 & ? & "، & I 55 & I 864 & 2 & ، \\
\hline 7 & Matehuala & “ & I 55 & I 864 & 2 & “ \\
\hline I I & ? & “" & 127 & I 864 & 8 & “ \\
\hline 30 & ? & “ & I 55 & I 864 & 2 & “ \\
\hline 37 & Matehuala & $" ،$ & I 27 & I 864 & I & “" \\
\hline 44 & Cedral & “ & I 27 & I 864 & I & “ \\
\hline
\end{tabular}

Series of Queretaro (State of Queretaro).

Surcharged with the extra number only, and also always with name of issuing district-Queretaro. The lowest number I have found surcharged is No. 4I, issued in I864, and I doubt if the consignments sent out previously were surcharged with the control number.

\begin{tabular}{|c|c|c|c|c|c|c|}
\hline $\begin{array}{l}\text { No. } \\
4 \mathrm{I}\end{array}$ & $?^{\text {Name. }}$ & surcharged on & $\begin{array}{l}\text { No. } \\
\text { I } 29\end{array}$ & $\begin{array}{l}\text { Date. } \\
\text { I } 864\end{array}$ & & $\begin{array}{l}\text { lue. } \\
\text { eales }\end{array}$ \\
\hline 43 & Celaya & .6 & 129 & I 864 & 2 & “، \\
\hline 44 & San Juan del Rio & “ & 129 & I 864 & 2 & “ \\
\hline 49 & Celaya & “ & $17 I$ & I 864 & 2 & “ \\
\hline 53 & Celaya & “ & I7I & I 864 & 2 & “ \\
\hline
\end{tabular}

Series of Puebla (State of Puebla).

Surcharged with the extra number only, and also always surcharged with the name of the issuing district-Puebla. The lowest number I have found surcharged is No. 44, in the 1864 issue.

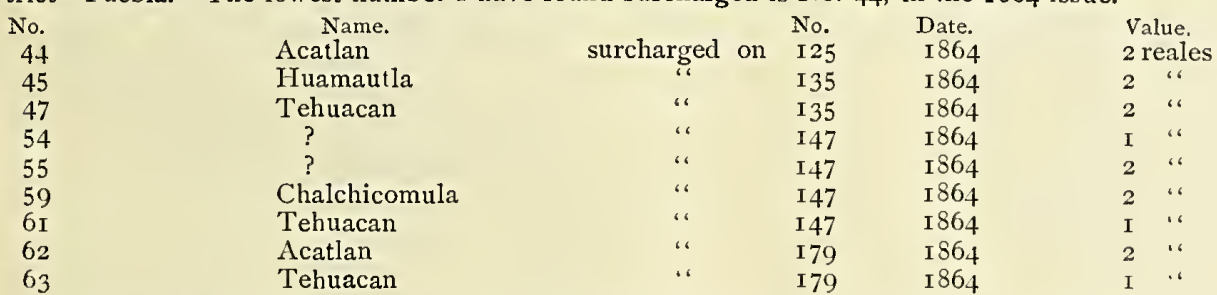

Series of Guanajuato (State of Guanajuato).

Surcharged with the extra number only, and also always with the name of issuing district-Guanajuato. The lowest number I have found is No. 53 in I864.

\begin{tabular}{|c|c|c|c|c|c|}
\hline $\begin{array}{r}\text { No. } \\
53\end{array}$ & $\begin{array}{l}\text { Name. } \\
\text { Penjama }\end{array}$ & sureharged on & $\begin{array}{l}\text { No. } \\
\text { I } 48\end{array}$ & $\begin{array}{l}\text { Date. } \\
\text { I } 864\end{array}$ & $\begin{array}{l}\text { Value. } \\
2 \text { reales }\end{array}$ \\
\hline & ? (Cancellation No. I) & $\because 6$ & I48 & I 864 & \\
\hline 58 & Irapuato & “" & I 48 & I 864 & 2 \\
\hline 59 & ? (Cancellation No. I) & “" & I48 & 1864 & 2 \\
\hline 60 & $?($ & “" & I 48 & I 864 & 2 \\
\hline 68 & Irapuato & “" & 176 & 1864 & 2 \\
\hline 69 & ? (Cancellation No. I) & “" & I 76 & I 864 & “" \\
\hline
\end{tabular}

It has been impossible to identify the offices using the following cancellations, which we illustrate in the hope that some of our readers may find them on entire letters and thus locate them.

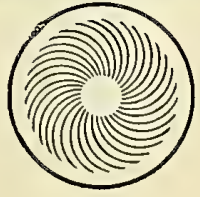

I.

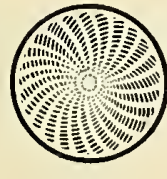

II. 
V. Surcharged with name of town ; number and date in small ordinary type.

$\mathbf{I}^{\circ}$ Vertically ribbed paper.

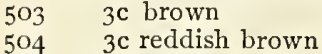

$505 \quad \mathrm{r} / 2$ (Medio) $\mathrm{r}$ lilac

$506 \quad 1 / 2$ (Medio) $r$ red lilac

$507 \quad 1 / 2$ (Medio) $r$ grayish lilac

508 I (un) blue gray (I 864 )

509 I (un) r ultramarine (I 864)

5IO I (un) r blue (I865)

5II I (un) r pale blue

5 I2 I (un) $r$ deep blue

$5 \mathrm{I} 3$ I (un) r Prussian blue

5 I4 I (un) r greenish blne

5 I5 2 (dos) $r$ yellow

5 I 62 (dos) $r$ orange

5 I 72 (dos) r red orange

5 I 84 (cuatro) $r$ green

5 I9 4 (cuatro) $r$ yellow green

5204 (cuatro) $r$ blue green

52 I 8 (ocho) $r$ red

522 - 8 (ocho) r pale red

$2^{\circ}$ Horizontally ribbed paper. 3 c brown

$3 \mathrm{c}$ reddish brown

$1 / 2$ (Medio) $r$ lilac

$1 / 2$ (Medio) r red lilac

$1 / 2$ (Medio) $r$ grayish lilac

I (un) $r$ blue gray (I864)

I (un) r ultramarine (I864)

I (un) $r$ blue ( 1865 )

I (un) $r$ pale blue

I (un) $r$ deep blue

I (un) r Prussian blue

I (un) $r$ greenish blue

2 (dos) r yellow

2 (dos) $r$ orange

2 (dos) $r$ red orange

4 (cuatro) r green

4 (cuatro) r yellow green

4 (cuatro) $r$ blue green

8 (ocho) $\mathrm{r}$ red

8 (ocho) pale red

$3^{\circ}$ Laid paper

$3 \mathrm{c}$ brown

$3 \mathrm{c}$ reddish brown

$1 / 2$ (Medio) $r$ lilac

$1 / 2$ (Medio) $r$ red lilac

$1 / 2$ (Medio) $r$ grayish lilac

I (un) $r$ blue gray (I864)

I (un) r ultramarine (I 864)

I (un) $r$ blue (I 865)

I (un) I pale blue

I (un) $r$ deep blue

I (un) r Prussian blue

I (un) $r$ greenish blue

2 (dos) r yellow

2 (dos) $r$ orange

2 dos) r red orange

4 (cuatro) r green

4 cuatro) $r$ pale green

4 (cuatro) $r$ blue green

8 (ocho) r pale red

$4^{\circ}$ Thick soft wove paper.

$3 \mathrm{c}$ brown

$3 \mathrm{c}$ reddish brown

$1 / 2$ (Medio) r lilac

$1 / 2$ (Medio) $r$ red lilac

$1 / 2$ (Medio) $r$ grayish lilac

I (un) $r$ blue gray (I864)

I (un) r ultramarine (I 864)

I (un) $r$ blue (I865)

I (un) $r$ pale blue

I (un) $r$ deep blue

I (un) $r$ Prussian blue

I (un) $r$ greenish blue

2 (dos) r yellow

2 (dos) $r$ orange

2 (dos) $r$ red orange

4 cuatro) $r$ green
5784 (cuatro) $r$ pale green

5794 (cuatro) $r$ blue green

$580 \quad 8$ (ocho) r red

$58 \mathrm{I} \quad 8$ (ocho) $\mathrm{r}$ pale red

$5^{\circ}$ Ordinary wove paper.

$582 \quad 3 \mathrm{c}$ brown

$583 \quad 3 \mathrm{c}$ reddish brown

$584 \quad 1 / 2$ (Medio) r lilac

$585 \quad 1 / 2$ (Medio) r red lilac

$586 \quad 1 / 2$ (Medio) r grayish lilac

587 I (un) r blue gray (I 864 )

588 I (un) r ultramarine (I 864)

$589 \quad$ I (un) $r$ blue ( 1865 )

590 I (un) r pale blue

59I I (un) r deep blue

502 I un $r$ Prussian blue

593 I (un) $r$ greenish blue

5942 (dos) r yellow

5952 (dos) $r$ orange

5962 (dos) r red orange

5974 (cuatro) r green

5984 (cuatro) $r$ pale green

5994 (cuatro) $r$ blue green

6008 (ocho) r red

6oI 8 (ocho) r pale red

Varieties:

a. 2 reales stamps cut vertically in two, each half being used as I real.

602 Ir orange (half of 2 reales), right half

603 Ir orange (half of 2 reales), left half

b. 2 reales stamps cut horizontally in two, each half being used as I real.

604 Ir orange (half of 2 reales), upper half

605 Ir orange (half of 2 reales), lower half

c. 2 reales stamps cut diagonally in two each half being used as I real.

606 Ir orange (half of 2 reales), upper right half

607 Ir orange (half of 2 reales), lower right half

608 Ir orange (half of 2 reales), upper left hif

609 Ir orange (half of 2 reales), lower left half

d. 4 reales stamps cut vertically in two each half being used as 2 reales.

6ro 2 r green (half of 4 reales), right half 6II 2 r green (half of 4 reales), left half

e. 4 reales stamps cut horizontally in two, each half being used as 2 reales.

6122 green (half of 4 reales), upper half

6I3 2r green (half of 4 reales), lower half

f. 4 reales stamps cut diagonally in two, each half being used as 2 reales.

$6 r_{4} 2$ r green (half of 4 reales), upper right half

6 I5 2r green (half of 4 reales), lower right half

6I6 2 r green (half of 4 reales), upper left half

$6 \mathrm{I} 72 \mathrm{r}$ green (half of 4 reales), lower left half

g. 8 reales stamps cut vertically in two each half being used as 4 reales.

6 I $84 \mathrm{r}$ red (half of 8 reales), right half

$6 \mathrm{rg} \quad 4 \mathrm{r}$ red (half of 8 reales), left half

h. 8 reales stamps cut horizontally in two, each half being used as 4 reales.

$620 \quad 4 \mathrm{r}$ green (half of 8 reales), upper half

$62 \mathrm{I} 4 \mathrm{r}$ green (half of 8 reales), lower half

i. 8 reales stamps cut diagonally in two, each half being used as 4 reales.

$6224 \mathrm{r}$ red (half of 8 reales), upper right half

$6234 \mathrm{r}$ red (half of 8 reales), lower right half

$6244 \mathrm{r}$ red (half of 8 reales), upper left half

$6254 \mathrm{r}$ red (half of 8 reales), lower left 


VI. Surcharged with number and date (small ordinary type) only.

$I^{\circ}$ Vertically ribbed paper. $3 c$ brown

$3 c$ reddish brown

$1 / 2$ (Medio) r lilac

$\pi / 2$ (Medio) $r$ red lilac

$1 / 2$ (Medio) r gray lilac

$\mathrm{I}$ (un) $\mathrm{r}$ blue gray (I864)

I (un) $r$ ultramarine (I864)

$\mathrm{I}$ (un) $\mathrm{r}$ blue (I865)

I. un $r$ pale blue

I (un) $r$ deep blue

I (un) $\mathbf{r}$ Prussian blue

$\tau$ (un) $r$ greenish blue

2 (dos) r yellow

2 (dos) $r$ orange

2 dos) $r$ red orange

4 (cuatro) r green

4 (cuatro) $r$ yellow green

4 (cuatro) $r$ blue green

8 ocho) r red

8 (ocho) $r$ pale red

$2^{\circ}$ Horizontally ribbed paper

$3 \mathrm{c}$ brown

$3 c$ reddish brown

$1 / 2$ (Medio) r lilac

$1 / 2$ (Medio) $x$ red lilac

$\pi / 2$ (Medio) $r$ gray lilac

I (un) blue gray (I 864)

I un r ultramarine (I864)

I (un) $r$ blue (I865)

I (un) $r$ pale blue

I (un) $r$ deep blue

I (un) r Prussian blue

I (un) I greenish blue

2 (dos) $r$ yellow

2 dos) $r$ orange

2 dos) $r$ red orange

4 (cuatro) $r$ green

4 cuatro) $r$ pale green

4 (cuatro) $r$ blue green

8 (ocho) r red

8 (ocho) r pale red

$3^{\circ}$ Laid paper

3c brown

3c reddish brown

$\mathrm{r} / 2$ (Medio) $\mathrm{r}$ lilac

$1 / 2$ (Medio) $\mathrm{r}$ red lilac

$1 / 2$ (Medio) $r$ gray lilac

I (un) $\mathbf{r}$ blue gray (I864)

I (un) $r$ ultramarine (I864)

I un) $r$ blue (I865)

$\mathrm{r}$ un $\mathrm{r}$ pale blue

I un) $r$ deep blue

I (un) $r$ Prussian blue

I (un) $x$ greenish blue

2 (dos) $r$ yellow

2 dos) $r$ orange

2 (dos) r red orange

4 (cuatro) r green

4 (cuatro) r yellow green

4 (cuatro) $r$ blue green

8 (ocho) $r$ red

8 (ocho) r pale red

$4^{\circ} 7$ hick soft wove paper.

$3 c$ brown

$3 \mathrm{c}$ reddish brown

$\pi / 2$ (Medio) $r$ lilac

$1 / 2$ (Medio) $r$ red lilac

$1 / 2$ (Medio) $r$ gray lilac

I un) $r$ blue gray (1864)

I (un) $r$ ultramarine (I864)

I (un) $r$ blue ( 1865 )

I (un) $r$ pale blue

I (un) $r$ deep blue

I (un) I Prussian blue

$\mathrm{I}$ (un) $\mathrm{r}$ greenish blue

2 (dos) r yellow

2 dos) $r$ orange

2 dos) $r$ red orange

4 (cuatro) r green
7024 (cuatro) $r$ yellow green

7034 (cuatro) $r$ blue green

7048 (ocho) r red

7058 (ocho) $\mathrm{r}$ pale red

$5^{\circ}$ Ordinary wove paper.

$706-3 \mathrm{c}$ brown

707 3c reddish brown

$708 \quad 1 / 2$ (Medio) $r$ lilac

$709 \quad 1 / 2$ (Medio) $r$ red lilac

7 ro $\quad 1 / 2$ (Medio) $r$ gray lilac

7 I I I (un) $r$ blue gray (I864)

$7 \mathrm{I2}$ I (un) $r$ ultramarine (I864)

7 I3 I (un) $r$ blue (I865)

7 I4 I un) $r$ pale blue

7 I 5 I (un) $r$ deep blue

7 I6 I (un) r Prussian blue

7I 7 I (un) $r$ greenish blue

7I8 2 (dos) $r$ yellow

7r9 2 (dos) $\mathrm{r}$ orange

$720 \quad 2$ (dos) $r$ red orange

$72 \pi \quad 4$ (cuatro) $r$ green

7224 (cuatro) $\mathrm{r}$ yellow green

7234 (cuatro) $r$ blue green

7248 (ocho) $r$ red

7258 (ocho) r pale red

a. 2 reales stamps cut vertically in two each half being used as I real.

726 Ir orange (half of 2 reales), right half

727 Ir orange (half of 2 reales), left half

b. 2 reales stamps cut horizontally in two, each half being used as I real.

728 Ir orange (half of 2 reales), upper half

729 Ir orange (half of 2 reales), lower half

c. 2 reales stamps cut diagonally in two, each half being used as 2 reales.

730 Ir orange (half of 2 reales), upper right half

73I Ir orange (half of 2 reales), lower right half

732 Ir orange (half of 2 reales), upper left half

733 Ir orange (half of 2 reales), lower left half

d. 4 reales stamps cut vertically in two, each half being used as 2 reales.

$734 \quad 2 \mathrm{r}$ green (half of 4 realea), right half

$735 \quad 2$ r green (half of 4 reales), left half

e. 4 reales stamps cut horizontally in two, each half being used as 2 reales.

$736 \quad 2 \mathrm{r}$ green (half of 4 reales), upper half

737 2r green (half of 4 reales), lower half

f. 4 reales stamps cut diagonally in two, each half being used as 2 reales.

738 2r green (half of 4 reales), upper right half

739 2r green (half of 4 reales), lower right half

$740 \quad 2$ r green (half of 4 reales), upper left half

74I 2r green (half of 4 reales), lower left half

g. 8 reales stamps cut vertically in two each half being used as 4 reales.

$742 \quad 4 \mathrm{r}$ red (half of 8 reales), right half

$7434 \mathrm{r}$ red (half of 8 reales), left half

h. 8 reales stamps cut horizontally in two,

each half being used as 4 reales.

$744 \quad 4 \mathrm{r}$ red (half of 8 reales), upper half

$745 \quad 4 \mathrm{r}$ red (half of 8 reales), lower half

i. 8 reales stamps cut diagonally in two, each half being used as 4 reales.

$7464 \mathrm{r}$ red (half of 8 reales), upper right half

$4 \mathrm{r}$ red (half of 8 reales), lower right half

748

$4 \mathrm{r}$ red (half of 8 reales), upper left half

$7494 \mathrm{r}$ red (half of 8 reales), lower left half 
The stamps surcharged with number and date only are with a few exceptions, seldom found used. Colima never surcharged these stamps; Campeche scarcely ever surcharged them; Jalapa and Zapotlan also frequently omitted to surcharge them.

List of District Names with Number and Date in Ordinary Type.

\begin{tabular}{|c|c|c|c|}
\hline District. & Meas, in $\mathrm{mm}$. & District. & Meas. in $\mathrm{mm}$. \\
\hline guascalientes & (Caillebotte) & -OAJACA. & I $5 \times 21 / 3$; bet. periods \\
\hline ICUASCALTES. & $22 \times 3$; with period & UAXACA & $141 / 2 \times 21 / 4$ \\
\hline LLENDE & $20 \times 3$ & ORI ZAVA. & I $71 / 2 \times 21 / 4 ;$ with period \\
\hline $\begin{array}{l}\text { PAM } \\
\text { RCA }\end{array}$ & $91 / 2 \times 2$ & ORIZAVA & $\begin{array}{l}\text { I } 71 / 2 \times 11 / 2 \text { to } 3 ; \text { with } \\
\text { period }\end{array}$ \\
\hline AMPECHE. & I $7 \times 2$; with period & O R I ZAVA & $161 / 2 \times 21 / 3$ \\
\hline OCULA & $12 \times 2$ & PACHUCA & I $3 \times 2$ \\
\hline ORDOVA. & I6x2; bet. periods & PACHUCA & $13 \frac{1}{2} \times 2$ \\
\hline uautitlan & (Caillebotte) & PAKRAL & I $5 \times 2 \mathrm{I} / 2$ \\
\hline $\begin{array}{l}\text { IERNAVACA. } \\
\text { IALCO }\end{array}$ & $\begin{array}{l}\text { I } 8 \times 2 \text {; with period } \\
\text { I } 3 \times 13 / 4\end{array}$ & QUERETARO & $\begin{array}{l}131 / 2 \times 21 / 3 ; \text { with period } \\
17 \times 17 / 8\end{array}$ \\
\hline HIHUAHUA & $22 \times 21 / 2$ & QUERETARO & $17 \times I 3 / 4$ \\
\hline URANGO & $15 \times 2$ & RIO FLORIDO & $22 \times 4$ \\
\hline ADALAJARA & $201 / 2 \times 21 / 4$ & SALTILLO & $15 \times 21 / 4$ \\
\hline ADALAJARA & $20 \times 2$ & & $15 \times 21 / 2$ \\
\hline TADALAJARA & $201 / 2 \times 21 / 2$ to $11 / 2$ & - SALTILLO• & I61/2XI $1 / 2$ bet. period \\
\hline JANAJUATO. & $23 \times 3$; with period & S.L.POTOSI. & I $7 \times 5$; with period \\
\hline JAYMAS & $14 \times 21 / 4$ & S.L.POTOSI & $17 \times 2$ \\
\hline IUEJUTLA & $16 \times 2$ & SOYANIQULLPAII & $20 \times 2$ \\
\hline UALA & $131 / 2 \times 2$ & TAMPICO & $131 / 2 \times 2$ \\
\hline “UALA & $\begin{array}{l}\text { I } 3 \frac{1}{1} 2 \times 2 \\
\text { I } 31 / 2 \times 2 ; \text { surc'ge in blue }\end{array}$ & TEPEJI & $\begin{array}{l}\text { I } 31 / 2 \times 13 / 4 \\
\text { I I X2 }\end{array}$ \\
\hline ALAPA & $101 / 2 \times 2$ & “ & $22 \times 2$; bet. periods \\
\hline AGOS. & I $41 / 2 \times 2$; with period & TEPIC & $71 / 2 \times 2$ \\
\hline GOS. & I $2 \times 2$; with period & TO L UCA & $12 \times 2$ \\
\hline LAGOS & IOX 2 & TULA & $8 \times 2$ \\
\hline MARAVATIO & $\begin{array}{l}\text { I } 8 \times 2 \\
\text { I } 8 \times 2 \text {; surcharge read- }\end{array}$ & TULANCINGO & I $8 \times 21 / 4$ \\
\hline & $\begin{array}{l}\text { ing backwards (Tap- } \\
\text { ling) }\end{array}$ & VERA CRUZ. & I6x2; bet. periods \\
\hline Elat & Surcharced in MS. & $\begin{array}{l}\text { VERACRUZ } \\
\text { VEAACRUZ }\end{array}$ & $\begin{array}{l}\text { I5 } 1 / 2 \times 11 / 2 \\
\text { I } 31 / 2 \times 11 / 2\end{array}$ \\
\hline ZATLAN & $\begin{array}{l}\text { surcharged in } M S \text {. } \\
171 / 2 \times 2\end{array}$ & VICTORLA & $\begin{array}{l}13 \frac{1}{2} \times 1 / 2 \\
12 \times 3\end{array}$ \\
\hline MERIDA & $13 \times 2$ & ZACATECAS. & $25 \times 3$; with period \\
\hline $\begin{array}{l}\text { SOPXXXeg } \\
\text { MONTERREY }\end{array}$ & $\begin{array}{l}15 \times 2 \\
191 / 2 \times 2\end{array}$ & $\begin{array}{l}\text { ZACATECAS. } \\
\text { ZACATECAS }\end{array}$ & $\begin{array}{l}\text { I9x2 } 1 / 2 ; \text { with period } \\
\text { I } 8 \times 2\end{array}$ \\
\hline IVtorelia & $161 / 2 \times 13 / 4$ & ZAPOTLAN & $14 \times 2$ \\
\hline
\end{tabular}

The following numbers will show where each consignment of the issues of 1864 to 1866 was sent, with number and date of ordinary type :

I 864 .

I80 Vera Cruz

I \& I Saltillo

I82 Apam

I83 Mexico

184 ?

185 Jalapa

I86 Morelia

I87 Toluca

I88 Mexico

189 S. L. Potosi

I90 Puebla

I9 I Orizava

I92 Mexico

193 Iguala

I94 Tampico

I95 Cordova

I96 Mexic

197 ?

I865.

Mexico

Vera Cruz

Mexico

Toluca

Mexico

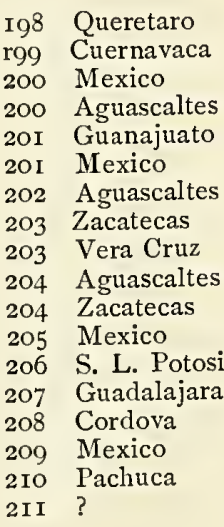

6 Mexico

7 Guanajuato

8 Puebla

9 Mexico

IO S. L. Potosi

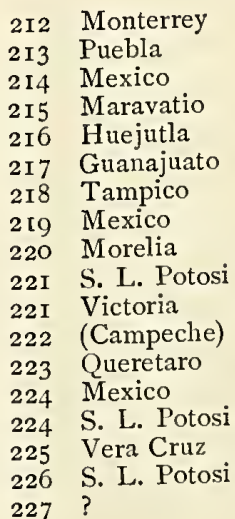

I Queretaro

I " with er-

ror in date 1885 .

12 Chalco

I3 Durango

$\begin{array}{ll}228 & \text { Apam } \\ 229 & \text { Mexico } \\ 230 & \text { Jalapa } \\ 231 & \text { ? } \\ \text { 232 } & \text { Saltillo } \\ 233 & \text { Durango } \\ 234 & \text { Mexico } \\ 235 & \text { Pachuca } \\ 236 & \text { Mazatlan } \\ 237 & \text { Mexico } \\ 238 & \text { Orizava } \\ 239 & \text { Mexico } \\ 240 & \text { Puebla } \\ 241 & \text { Queretaro } \\ 242 & \text { Vera Cruz } \\ 243 & \text { Guadalajara } \\ \text { 244 } & \text { Zacatecas } \\ \text { 244 } & \text { Mexico } \\ & \\ & \\ \text { I3 } & \text { Morelia } \\ \text { I4 } & \text { Tulancingo } \\ \text { I5 } & \text { Mexico } \\ \text { I5 } & \text { Tepic } \\ \text { I6 } & \text { Mexico }\end{array}$





\begin{tabular}{|c|c|c|c|c|c|c|c|}
\hline I7 & Mexico & 69 & Mexico & $\mathbf{I} 24$ & Pachuca & I 72 & S. L. Potosi \\
\hline 17 & Morelia & 70 & Mazatlan & I25 & Mexico & I73 & Saltillo \\
\hline 18 & Monterey & $7 I$ & Mexico & I 26 & Mexico & 174 & Queretaro \\
\hline I 8 & Zacatecas & 72 & Orizava & 127 & Mexico & 175 & $?$ \\
\hline $2 I$ & Lagos & 73 & Pachuca & I 28 & Mexico & 176 & Toluca \\
\hline 22 & $?$ & 73 & Saltillo & 129 & Queretaro & 177 & Mexico \\
\hline 23 & Mexico & 74 & Mexico & 130 & Guanajuato & 178 & Vera Cruz \\
\hline 24 & Cocula & 75 & $?$ & 131 & Cocula & I 79 & Orizava \\
\hline 24 & Colima & 76 & Mexico & 131 & Guadalajara & 180 & Colima \\
\hline 24 & Guadalajara & 77 & Mexico & 131 & Puebla & I 81 & $?$ \\
\hline 24 & Zapotlan & 78 & Huejutla & I3I & Zapotlan & I 82 & Mexico \\
\hline 25 & Apam & 79 & Apam & 132 & Mexico & I 83 & Yguala \\
\hline 26 & Guanajuato & 80 & Colima & 133 & Mexico & 183 & Mexico \\
\hline 27 & Saltillo (2 types) & $8 I$ & Maravatio & 134 & Merida & I 84 & Queretaro \\
\hline 28 & Yguala & 82 & Mexico & 135 & S. L. Potosi & I 85 & Cuernavaca \\
\hline 29 & Merida & 82 & S. L. Potosi & 135 & Matamoros & I 86 & Apam \\
\hline 30 & Mexico & 83 & S. L. Potosi & & (written) & I 87 & Mexico \\
\hline $3 I$ & I del Carmen & 84 & Morelia & 136 & Mexico & I 88 & Apam \\
\hline $3 I$ & Vera Cruz & 85 & Mexico & 137 & Guadalajara & I 89 & Cordova \\
\hline 32 & Orizava & 86 & Queretaro & 138 & Puebla & Igo & Puebla \\
\hline 33 & Queretaro & 87 & Yuebla & I39 & S. L. Potosi & 191 & Mazatlan \\
\hline 34 & $?$ & 88 & Oaxaca & 140 & Orizava & I92 & Maravatio \\
\hline 35 & Orizava & 89 & Cordova & I 40 & " with & 192 & Mexico \\
\hline 36 & Mexico & 89 & Vera Cruz & \multicolumn{2}{|c|}{ error in date $\mathbf{1} 885$} & 193 & Mexico \\
\hline 37 & Puebla & 90 & Mexico & I4I & Durango & I 94 & Guanajuato \\
\hline 38 & Queretaro & $9 \mathrm{I}$ & Toluca & 142 & Mexico & 195 & Chalco \\
\hline 39 & Cuernavaca & 92 & Cocula & 143 & Morelia & I 96 & Vera Cruz \\
\hline 40 & Oaxaca & 92 & Guadalajara & I43 & " with er- & 197 & Mexico \\
\hline 40 & Oaxaca & $9^{2}$ & Tepic & ror & n date $865 \mathrm{I}$ & 198 & Mexico \\
\hline $4 I$ & S. L. Potosi & 92 & Zapotian & I 44 & Cordova & 199 & Pachuca \\
\hline 42 & Jalapa & 93 & Durango & 145 & Cuernavaca & 200 & Tampico \\
\hline 43 & Mexico & 94 & Vera Cruz & 146 & Jalapa & $2 O I$ & Orizava \\
\hline 44 & Maravatio & 95 & Cordova & 146 & " without & 202 & $?$ \\
\hline 45 & Mexico & 96 & Apam & & date & 203 & Lagos \\
\hline 46 & Pachuca & 97 & Mexico & I 47 & Mexico & 204 & ? \\
\hline 47 & Toluca & 98 & Mexico & 148 & Zacatecas & 205 & Tulancingo \\
\hline 48 & Mexico & 99 & Mexico & I 49 & Queretaro & 206 & Mexico \\
\hline 49 & Mexico & IOO & Jalapa & 150 & Mexico & 206 & S. L. Potosi \\
\hline 50 & ? & IOI & Guanajuato & I 1 & Pachuca & 207 & Tepeji \\
\hline $5 \mathrm{I}$ & Cordova & 102 & Zacatecas & I 52 & Vera Cruz & 208 & S. L. Potosi \\
\hline $5 I$ & Mexico & 103 & Chalco & I53 & Cuautitlan & 209 & Chalco \\
\hline 53 & Guanajuato & IO4 & Mexico & I 54 & Mexico & 210 & Mexico \\
\hline 53 & Vera Cruz & 105 & Mexico & I 55 & Guadalajara & $2 \mathrm{II}$ & Guadalajara \\
\hline 54 & Mexico & I06 & Mexico & 155 & Tepic & $21 I$ & Tepic \\
\hline 55 & Guadalajara & 107 & Pachuca & I55 & Zapotlan & $2 \mathrm{II}$ & Zapotlan \\
\hline 55 & Colima & I08 & Oaxaca & I 56 & Puebla & 212 & Cuernavaca \\
\hline 55 & Tepic & IO9 & Morelia & I 57 & Mexico & 213 & Mexico \\
\hline 55 & Zapotlan & I IO & S. L. Potosi & I58 & Guanajuato & 213 & Puebla \\
\hline 56 & Puebla & I IO & “ with & I 59 & Oaxaca & $2 \mathrm{I} 4$ & Toluca \\
\hline 57 & Queretaro & & error in date, & 160 & ? & 215 & Guanajuato \\
\hline 58 & Guanajuato & & I 868. & I6I & ? & 216 & Puebla \\
\hline 59 & Oaxaca & I I I & Mexico & 162 & Mexico & 217 & ? \\
\hline 59 & Zacatecas & II 2 & $?$ & I 63 & Tampico & 218 & Mexico \\
\hline 60 & Mexico & II3 & Tulancingo & 164 & Campeche & 219 & $?$ \\
\hline $6 \mathbf{I}$ & Tula & I I4 & Orizava & I65 & ? & 220 & ? \\
\hline 62 & Tampico & II 5 & Puebla & 166 & $?$ & $22 I$ & $?$ \\
\hline 63 & Monterey & I I 6 & Guaymas & 167 & Pachuca & 222 & $?$ \\
\hline 64 & S. L. Potosi & I I 7 & Jalapa & I68 & Lagos & 223 & $?$ \\
\hline 64 & Zacatecas & I I 8 & Mexico & I69 & Allende & 224 & ? \\
\hline 65 & Mexico & II 9 & Vera Cruz & 169 & Chihuahua & 225 & Vera Cruz \\
\hline 66 & $?$ & 120 & Mexico & 169 & Parral & & \\
\hline 67 & Cuernavaca & I 2 I & Yguala & 169 & Rio Florido & & \\
\hline 67 & Pachuca & 122 & Huejutla & $\mathbf{I} 70$ & Guanajuato & & \\
\hline 68 & Soyaniquilpam & I 23 & Toluca & I 71 & Mexico & & \\
\hline & I 866. & & & & & & \\
\hline I & Mexico & 12 & Orizava & $2 \mathrm{I}$ & Tulancingo & 33 & Queretaro \\
\hline 2 & Orizava & 13 & Cordova & 22 & Puebla & 34 & Saltillo \\
\hline 3 & Mexico & 14 & Lagos & 23 & Jalapa & 35 & Mexico \\
\hline 4 & Guanajuato & I 5 & Apam & 24 & Zacatecas & 36 & ? \\
\hline 5 & Mexico & I6 & Morelia & 25 & Mexico & 37 & Puebla \\
\hline 5 & Zacatecas & I7 & Mexico & 26 & Vera Cruz & 37 & Toluca \\
\hline 6 & Vera Cruz & 18 & Merida & 26 & Guadalajara & 38 & Vera Cruz \\
\hline 7 & Mexico & I9 & Jalapa & 27 & Mexico & 39 & Lagos \\
\hline 8 & Jalapa & I0 & Mexico & 28 & S. L. Potosi & 40 & Mexico \\
\hline 8 & Mexico & 20 & Cocula & 29 & ? & $4 I$ & Cordova \\
\hline 9 & Tampico & 20 & Guadalajara & 30 & Puebla & 42 & Oaxaca \\
\hline IO & Durango & 20 & Tepic & $3 I$ & Mexico & 43 & Monterrey \\
\hline I I & Guanajuato & 20 & Zapotlan & 32 & Orizava & 43 & (Colima) \\
\hline
\end{tabular}




$\begin{array}{ll}44 & \text { (Colima) } \\ 45 & \text { Guanajuato } \\ 46 & \text { S. L. Potosi } \\ 46 & \text { Mexico } \\ 47 & \text { ? } \\ 48 & \text { Mexico } \\ 49 & \text { Zacatecas } \\ 50 & ? \\ 51 & \text { Guadalajara } \\ 51 & \text { Tepic } \\ 51 & \text { Zapotlan } \\ 52 & \text { Mexico } \\ 53 & \text { Puebla } \\ 54 & \text { Zapotlan } \\ 55 & \text { Mexico } \\ 56 & \text { Mexico } \\ 57 & \text { S. L. Potosi } \\ 58 & \text { Vera Cruz } \\ 59 & \text { Mexico } \\ 60 & \text { Guanajuato } \\ 61 & \text { Guaymas } \\ 62 & \text { Mazatlan } \\ 63 & \text { ? } \\ 64 & \text { Mexico } \\ 65 & \text { Queretaro }\end{array}$

$\begin{array}{ll}66 & \text { Morelia } \\ 67 & \text { Durango } \\ 68 & \text { Mexico } \\ 69 & \text { Jalapa } \\ 70 & \text { Orizava } \\ 71 & ? \\ 72 & \text { Mexico } \\ 73 & \text { Pachuca } \\ 74 & \text { Pachuca } \\ 75 & \text { Vera Cruz } \\ 76 & \text { Chalco } \\ 77 & \text { S. L. Potosi } \\ 78 & \text { Mexico } \\ 78 & \text { Vera Cruz } \\ 79 & \text { Toluca } \\ 80 & ? \\ 81 & \text { Mexico } \\ 82 & \text { Zacatecas (two } \\ & \text { types) } \\ 83 & ? \\ 84 & ? \\ 85 & \text { Apam } \\ 86 & \text { Mexico } \\ 87 & ? \\ 88 & \text { Guanajuato } \\ & \end{array}$

\begin{aligned} & 89 Mexico \\ & 90 Merida \\ & 91 Cuernavaca \\ & 91 Vera Cruz \\ & 92 Guadalajara \\ & 92 Tepic \\ & 92 Zapotlan \\ & 93 Mexico \\ & 94 Vera Cruz \\ & 95 Oaxaca \\ & 96 Maravatio \\ & 97 S. L. Potosi \\ & 98 Cuernavaca \\ & 99 Tulancingo \\ & I00 Mexico \\ & I0I Queretaro \\ & I02 Puebla \\ & I03 Orizava \\ & I04 Vera Cruz \\ & 105 Mexico \\ & I06 Mexico \\ & I06 Tlalpujahua \\ & I07 Guanajuato \\ & I08 Mexico \\ & I09 Mexico \\ & \hline\end{aligned}

Sub-Districts.

Series of Guadalajara (State of Jalesco).

\begin{tabular}{|c|c|c|}
\hline No. & Date. & Used in \\
\hline I I & I 864 & (Tepic) \\
\hline I 2 & I 864 & ") \\
\hline I3 & 1865 & Cocula \\
\hline$I_{4}$ & I 865 & Tepic \\
\hline I5 & I865 & Zapotlan \\
\hline \multirow{16}{*}{ I 865} & No. & Used in \\
\hline & I5 & (Tepic) \\
\hline & $\begin{array}{l}15 \\
16\end{array}$ & $\begin{array}{l}\text { (Colima) } \\
\text { (Tepic) }\end{array}$ \\
\hline & 18 & Cocula \\
\hline & 19 & (Zapotlan) \\
\hline & 20 & (Colima) \\
\hline & 22 & $($ ( " $)$ \\
\hline & $\begin{array}{l}23 \\
23\end{array}$ & Zapotlan \\
\hline & 24 & Tepic \\
\hline & 9 & Cocula \\
\hline & 32 & Zapotlan \\
\hline & 32 & \\
\hline & 33 & Tepic \\
\hline & 42 & $\begin{array}{l}\text { Lapotian } \\
\text { Tepic }\end{array}$ \\
\hline & 42 & \\
\hline & 43 & Zapotlan \\
\hline \multirow[t]{11}{*}{ I 866} & I & Tepic \\
\hline & 2 & Zapotlan \\
\hline & 3 & Tepic \\
\hline & 4 & Cocula \\
\hline & 5 & Zapotlan \\
\hline & 6 & Tepic \\
\hline & 7 & Cocula \\
\hline & II & $\begin{array}{l}\text { Tepic } \\
\text { Zapotlan }\end{array}$ \\
\hline & I 2 & $\begin{array}{l}\text { Zapotian } \\
\text { (Tepic) }\end{array}$ \\
\hline & I 2 & $(4)$ \\
\hline & I6 & Tepic \\
\hline
\end{tabular}

Surcharged with number and date.

\begin{tabular}{|c|c|c|c|c|}
\hline surcharged on & $\begin{array}{l}\text { No. } \\
207\end{array}$ & $\begin{array}{l}\text { Date. } \\
\text { I } 8664\end{array}$ & & $\begin{array}{l}\text { Value. } \\
\text { reales }\end{array}$ \\
\hline$"$ & 243 & I 864 & 2 & ? \\
\hline " & 243 & I 864 & $1 / 2 \& 2$ & “ \\
\hline “ & 243 & I 864 & 2 & " \\
\hline “" & 243 & I 864 & $1 / 2$ & " \\
\hline
\end{tabular}

Surcharged with number only.

$\begin{array}{ll}\text { I09 } & \text { Morelia } \\ \text { I10 } & \text { Mexico } \\ \text { I I } & \text { S. L. Potısi } \\ \text { I12 } & \text { Orizava } \\ \text { I 13 } & \text { Puebla } \\ \text { I14 } & \text { Mexico } \\ \text { I15 } & \text { Puebla } \\ \text { I16 } & \text { Tulancingo } \\ \text { I I } & \text { Mexico } \\ \text { I } 8 & \text { Puebla } \\ \text { I } 19 & \text { Guanajuato } \\ \text { I20 } & \text { Vera Cruz } \\ \text { I21 } & \text { Queretaro } \\ \text { I22 } & \text { Colima) } \\ \text { I23 } & \text { ? } \\ \text { I24 } & \text { S. L. Potosi } \\ \text { I25 } & \text { Vera Cruz } \\ \text { I26 } & \text { Mexico } \\ \text { I27 } & \text { Mexico } \\ \text { I28 } & \text { Jalapa } \\ \text { I29 } & \text { ? } \\ \text { I30 } & \text { ? } \\ \text { I3 } & \text { Guadalajara } \\ \end{array}$

\begin{tabular}{|c|c|c|c|c|}
\hline & No. & Date. & & alue. \\
\hline surcharged on & 24 & I 865 & I \& 2 & eales \\
\hline & 24 & 1865 & $I \& 2$ & “ \\
\hline “" & 24 & I 865 & 2 & “. \\
\hline “ & 24 & I 865 & I \& 2 & " \\
\hline “. & 24 & 1865 & 2 & ". \\
\hline “ & 24 & 1865 & I \& 2 & " \\
\hline “" & 55 & 1865 & I \& 2 & “ \\
\hline “" & 24 & 1865 & $1 / 2 \& I$ & " \\
\hline & 55 & 1865 & I & \\
\hline “" & 55 & 1865 & 2 & ' \\
\hline “" & 92 & 1865 & 2 & \\
\hline “ & 92 & 1865 & 2 & “" \\
\hline “" & 55 & 1865 & I & “" \\
\hline “" & 92 & 1865 & 2 & “" \\
\hline “" & I3I & 1865 & I & “ \\
\hline “" & I3I & 1865 & I & “ \\
\hline " & I 55 & 1865 & I \& 2 & “ \\
\hline “ & I 55 & 1865 & I & - \\
\hline “" & I 55 & I 865 & 2 & “ \\
\hline ." & 2 I I & 1865 & 2 & “ \\
\hline “، & 2 II & I 865 & $1 / 2 \& 2$ & ' \\
\hline “" & 20 & I 866 & 2 & " \\
\hline “" & 20 & I 866 & 2 & “" \\
\hline “" & 20 & I 866 & 2 & “ \\
\hline " & 20 & I 866 & I \& 2 & "“ \\
\hline “" & 20 & I 866 & $1 / 2 \& 2$ & “ \\
\hline " & $5 \mathrm{I}$ & I 866 & 2 & . \\
\hline “" & 92 & I 866 & 2 & “ \\
\hline “. & $5 I$ & I 866 & I & " \\
\hline “" & 92 & I 866 & 2 & “" \\
\hline “" & $5 \mathrm{I}$ & I 866 & I & “ \\
\hline
\end{tabular}

Series of Jalapa (State of Vera Cruz).

This series is always surcharged with the date as well as the control number, the system of surcharging did not commence until sometime in $\mathbf{I} 865$, and the stamps always bear the name of the issuing district-Jalapa but not the name of the town to which they were consigned.

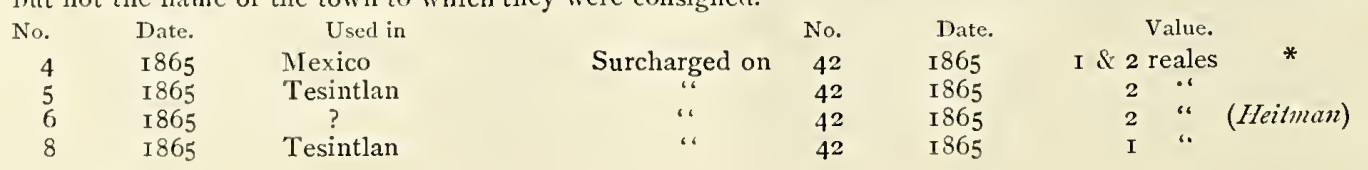






\begin{tabular}{|c|c|c|c|c|c|c|c|c|}
\hline $\begin{array}{r}\text { No. } \\
8\end{array}$ & $\begin{array}{l}\text { Date. } \\
\text { I } 865\end{array}$ & $\begin{array}{l}\text { Used in. } \\
\text { Tesiutlan }\end{array}$ & surcharged on & $\begin{array}{l}\text { No. } \\
\text { roo }\end{array}$ & $\begin{array}{l}\text { Date. } \\
\text { I } 865\end{array}$ & I \& 4 & $\begin{array}{l}\text { lue. } \\
\text { eales }\end{array}$ & \\
\hline IO & I 865 & if & is & IOO & I 865 & 2 & ", & \\
\hline II & 1865 & Perote & "6 & IOO & I 865 & 2 & ، & \\
\hline 14 & I 865 & $?$ & $" ،$ & I 46 & I 865 & 2 & “ & \\
\hline 15 & I 865 & ? & $"$ " & I46 & I 865 & 2 & ، & \\
\hline 15 & 1865 & ? & “ & IOO & 1865 & 2 & “ & (Heitman) \\
\hline I5 & I 865 & $?$ & “ & I I 7 & 1865 & 2 & “6 & (Ileitman) \\
\hline 16 & 1865 & Altotonga & “" & 146 & I 865 & 2 & “ & \\
\hline I & I 866 & Altotonga & ' & 8 & I 866 & 2 & "6 & \\
\hline 2 & I 866 & ? & $"$ & 8 & I 866 & $1 / 2$ and 4 & " & \\
\hline 2 & I 866 & ? & “ & +2 & 1866 & 8 & " & \\
\hline 4 & I 866 & ? & “" & 8 & I 866 & $1 / 2, I$ and 2 & " & \\
\hline $\begin{array}{l}4 \\
5\end{array}$ & I 866 & Manantial & $"$ " & 8 & I 866 & $1 / 2$ and & “ & \\
\hline 6 & I 866 & Papantla & ‘ & 8 & I 866 & 2 & $\because 6$ & \\
\hline 7 & I 866 & Altotonga & "، & 23 & I 866 & 2 & $\therefore$ & \\
\hline 8 & 1866 & ? & ‘. & 23 & I 866 & I & ، & (IFeitman) \\
\hline 9 & 1866 & Papantla & " & 69 & I 866 & 2 & ‘ & \\
\hline Io & I 866 & Tesiutlan & $\because$ & 69 & I 866 & 2 & " & \\
\hline 12 & I 866 & Altotonga & “6 & 69 & I 866 & 2 & "6 & \\
\hline 13 & I 866 & ? & "6 & 69 & I 866 & 2 & " & \\
\hline 14 & I $8 \mathrm{C} 6$ & Perote & 16 & I 28 & I 866 & 2 & “" & \\
\hline
\end{tabular}

* These may possibly be considered as veritable Habilitados. Tesiutlan is in the state of Puebla, Altotonga I have been unable to place; the other towns except of course Mexico, are in the state of Vera Cruz.

Series of Zacatecas (State of Zacatecas).

A large part of these siamps were consigned to Aguas Callientes, a very small state on the border of Zacatecas and were almost always sent without surcharge; on reaching there they were surcharged "Aguascaltes". In the table following it is to be understood that when the name $\Lambda$ guascaltes is bracketed, the stamps already bore the name Zacatecas. The consignments sent to the other towns were always surcharged Zacatecas only.

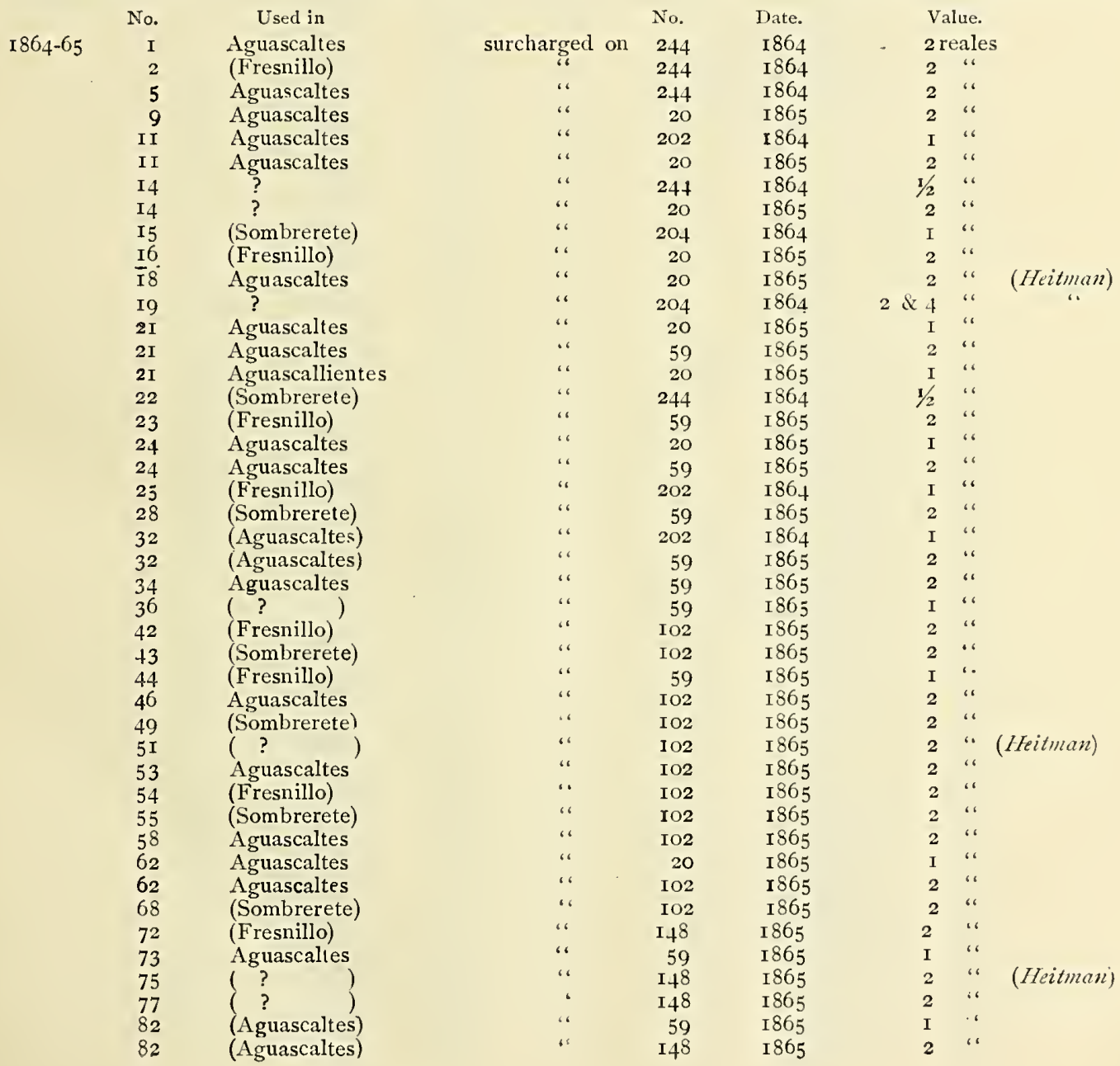




\begin{tabular}{|c|c|c|c|c|c|c|c|c|}
\hline \multirow{20}{*}{ I 866} & \multirow{3}{*}{$\begin{array}{r}\text { No. } \\
2 \\
3\end{array}$} & \multirow{4}{*}{$\begin{array}{c}\text { Used in } \\
\text { Aguascaltes } \\
\left(\begin{array}{c}? \\
\text { (Fresnillo) }\end{array}\right.\end{array}$} & \multirow{3}{*}{ surcharged on } & No. & \multirow{3}{*}{$\begin{array}{l}\text { Date. } \\
\text { I } 866 \\
\text { I } 866\end{array}$} & \multicolumn{2}{|c|}{ Value. } & \\
\hline & & & & 5 & & & eales & \\
\hline & & & & 5 & & 2 & $" 6$ & \\
\hline & 4 & & ، & 5 & I 866 & 2 & “ & \\
\hline & 7 & Aguascaltes & “" & 204 & 1864 & 4 & $"$ " & \\
\hline & 7 & Aguascaltes & “ & 59 & 1865 & $\mathbf{I}$ & “ & (Heitman) \\
\hline & 12 & Aguascaltes & ‘ & 24 & I 866 & 2 & “ & \\
\hline & 17 & Aguascaltes & “ & 24 & I 866 & 2 & " & \\
\hline & 23 & Aguascaltes & "، & 24 & I 866 & 2 & “ & \\
\hline & 26 & $(?)$ & " & 24 & I 866 & 2 & “ & \\
\hline & 28 & Aguascaltes & “، & 24 & I 866 & 2 & “. & \\
\hline & 30 & (Fresnillo) & “، & 24 & I 866 & 2 & “ & \\
\hline & $3 I$ & (Mexico) & "“ & 49 & I 866 & 2 & ،* & \\
\hline & 32 & Aguascaltes & $\because$ & 49 & I 866 & 2 & . & \\
\hline & 35 & Aguascaltes & ، & $\begin{array}{l}49 \\
24\end{array}$ & I 866 & 2 & “، & (Heitman) \\
\hline & 37 & $10 ?$ & “ & 24 & I 866 & I & ، & \\
\hline & 40 & Aguascaltes & " & 49 & I 866 & 2 & “ & \\
\hline & 45 & Aguascaltes & "، & 24 & I 866 & 2 & “ & (Ileitman) \\
\hline & 46 & Aguascaltes & “ & 82 & I 866 & 2 & " & \\
\hline & 47 & Aguascaltes & "، & 82 & I 866 & 2 & “" & \\
\hline
\end{tabular}

* These may possibly be considered as veritable Habilitados.

Series of S. L, Potosi (State of San Luis Potosi).

\begin{tabular}{|c|c|c|c|c|c|c|c|}
\hline \multirow{20}{*}{ I 864} & No. & $\begin{array}{c}\text { Used in } \\
\text { Matehuala }\end{array}$ & \multirow{3}{*}{$\begin{array}{c}\text { surcharged on } \\
\text { ، }\end{array}$} & \multirow{2}{*}{$\begin{array}{l}\text { No. } \\
221 \\
\text { I } 89\end{array}$} & \multirow{2}{*}{$\begin{array}{l}\text { Date. } \\
\text { I } 864 \\
\text { I } 864\end{array}$} & \multirow{2}{*}{\multicolumn{2}{|c|}{ Value. }} \\
\hline & $\begin{array}{l}33 \\
35\end{array}$ & Moctezuma & & & & & \\
\hline & 38 & $?$ & & 221 & I 864 & I & ، \\
\hline & 39 & Ciudad del Maiz & “ & I89 & I864 & 2 & “ \\
\hline & $4 \mathrm{I}$ & Catorce & “" & 189 & I864 & 2 & " \\
\hline & 54 & Catorce & “" & 206 & $1864^{\circ}$ & 2 & “" \\
\hline & 55 & $?$ & “ & 206 & 1864 & 2 & “" \\
\hline & 56 & Matehuala & “ & 206 & I 864 & 2 & “ \\
\hline & 57 & ? & “ & 206 & 1864 & 2 & ،. \\
\hline & 58 & Tula de Tamaulipas & “" & 206 & I $864^{\circ}$ & 2 & “" \\
\hline & $6 I$ & Salinas & “" & 206 & 1864 & 2 & “" \\
\hline & 62 & Tula de Tamaulipas & “" & 206 & 1864 & I & “ \\
\hline & 62 & Tula de Tamaulipas & “ & 226 & 1864 & 2 & “ \\
\hline & 64 & Ciudad del Maiz & “ & 226 & 1864 & 2 & “ \\
\hline & 65 & $?$ & “" & 226 & $1864^{\circ}$ & 2 & “ \\
\hline & 67 & ? & “ & 206 & I 864 & I & “" \\
\hline & 67 & $?$ & “ & 226 & 1864 & 2 & “" \\
\hline & 68 & Catorce & “" & 226 & I 864 & 2 & " \\
\hline & 70 & ? & “" & 206 & I 864 & I & “" \\
\hline & 74 & ? & “ & 226 & 1864 & 2 & “ \\
\hline \multirow[t]{34}{*}{ I 865} & 2 & Catorce & “ & Io & I 865 & 2 & "“ \\
\hline & 4 & $?$ & “ & IO & J 865 & $1 / 2 \& I$ & “ \\
\hline & 5 & Matehuala & “" & Io & I 865 & I & “" \\
\hline & IO & Catorce & “ & Io & 1865 & 2 & " \\
\hline & I I & Tula de Tamaulipas & “ & IO & 1865 & $1 / 2, I \& 2$ & “" \\
\hline & I3 & Catorce & “" & Io & 1865 & 2 & “" \\
\hline & I4 & GuadaIcazar & “" & Io & 1865 & 2 & “" \\
\hline & 16 & Matehuala & “" & Io & I 865 & I \& 2 & " \\
\hline & I 8 & Catorce & “" & IO & 1865 & 2 & “" \\
\hline & 20 & Salinas & “" & IO & 1865 & 2 & “" \\
\hline & $2 \mathrm{I}$ & Tula de Tamaulipas & “. & IO & 1865 & I \& 2 & “” \\
\hline & 23 & Matehuala & “" & $4 \mathrm{I}$ & I865 & I \& & “ \\
\hline & 24 & Ciudad del Maiz & “" & $4 \mathrm{I}$ & 1865 & 2 & “ \\
\hline & 26 & Tula de Tamaulipas & “" & $4 \mathrm{I}$ & 1865 & 2 & “" \\
\hline & 29 & Matehuala & “" & Io & $1865^{\circ}$ & 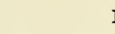 & “" \\
\hline & 29 & Matehuala & “ & $4 \mathrm{I}$ & 1865 & 1 & “ \\
\hline & 30 & Salinas & " & $4 I$ & 1865 & 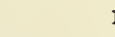 & “ \\
\hline & 33 & $?$ & “" & 64 & I 865 & 2 & “" \\
\hline & 34 & Matehutala & “. & 64 & 1865 & 2 & “" \\
\hline & 35 & Cedral & ". & IO & 1865 & 1 & “ \\
\hline & 36 & $?$ & “" & 64 & I 865 & 1 & “ \\
\hline & 37 & Rio Verde & “. & 64 & I865 & 2 & “* \\
\hline & 37 & Mexico & “" & 64 & 1865 & & “. \\
\hline & $3^{8}$ & Salinas & “" & 64 & 1865 & 2 & “ \\
\hline & 39 & Tula de Tamaulipas & “" & 64 & I865 & 2 & “" \\
\hline & $4 I$ & Matehuala & “ & 64 & 1865 & 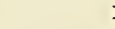 & “ \\
\hline & $4 \mathrm{r}$ & Matehuala & “ & $4 I$ & I865 & 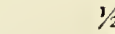 & “ \\
\hline & 43 & Ciudad del Maiz & “" & 64 & I865 & & “ \\
\hline & 45 & Matehuala & “" & 64 & I 865 & 2 & “" \\
\hline & 48 & Matehuala & “" & 83 & 1865 & & “” \\
\hline & 50 & Mexico & “" & 83 & I 865 & 2 & “* \\
\hline & $5 \mathrm{I}$ & ? & “" & 83 & 1865 & 2 & “" \\
\hline & 53 & Matehuala & “" & I ro & 1865 & 1 & “ \\
\hline & $5^{6}$ & Matehuala & “" & 6.4 & J 865 & 2 & " \\
\hline
\end{tabular}





\section{,}


MEXICO.

\begin{tabular}{|c|c|c|c|c|c|c|c|}
\hline \multirow{27}{*}{ I 865} & No. & Used in & & No. & Date. & \multicolumn{2}{|c|}{ Value. } \\
\hline & 56 & Matehuala & surcharged on & 83 & I 865 & I & real \\
\hline & 57 & Catorce & a & 83 & 1865 & 2 & $"$. \\
\hline & 60 & Charcas & “ & I IO & I 865 & I \& 2 & “ \\
\hline & $6 \mathbf{I}$ & Matehuala & “6 & I IO & I 865 & 2 & “‘ \\
\hline & 62 & Salinas & “ & I IO & I 865 & 2 & ، \\
\hline & 63 & Matehuala & ، & I IO & I 865 & I & “. \\
\hline & 65 & Mazapil ? & ، & I IO & I 865 & 2 & “ \\
\hline & 66 & Rio Verde & “ & I IO & 1865 & 2 & “ \\
\hline & 69 & Charcas & ، & IIO & $\mathrm{r} 865$ & 2 & ، \\
\hline & 70 & Ciudad del Maiz & “" & I IO & I 865 & 2 & “ \\
\hline & 70 & Ciudad del Maiz & “ & 83 & I 865 & I & “‘ \\
\hline & 71 & Moctezuma & ، & I 39 & I 865 & 2 & “ \\
\hline & 72 & Matehuala & “ & 83 & I 865 & I & “ \\
\hline & 77 & Matehuala & “6 & 83 & I 865 & I & “ \\
\hline & 82 & Matehuala & “" & 139 & I 865 & I \& 2 & “ \\
\hline & 83 & Salinas & “ & I 39 & 1865 & 2 & “ \\
\hline & 87 & ? & ، & I39 & I 865 & I & “ \\
\hline & 89 & Matehuala & “. & I72 & 1865 & 2 & “ \\
\hline & 90 & Rio Verde & “ & 83 & I 865 & I & “ \\
\hline & 94 & Matehuala & “ & I39 & I 865 & I & “ \\
\hline & 96 & Matehuala & ، & 172 & 1865 & 4 & “. \\
\hline & 97 & Salinas & “ & I 72 & I 865 & 2 & “ \\
\hline & 69 & Charcas & ، & 172 & 1865 & 2 & ، \\
\hline & 99 & Charcas & ، & 139 & I 865 & I & ، \\
\hline & I03 & Catorce & “ & I72 & I 865 & 2 & “. \\
\hline & 120 & Ciudad del Maiz & “ & I72 & I 865 & 2 & “ \\
\hline I 866 & I & Tula de T & “ & 208 & I 865 & 2 & “ \\
\hline & 2 & Matehuala & “، & I 39 & I 865 & I & “ \\
\hline & 3 & Matehuala & “6 & 208 & I 865 & 2 & ، \\
\hline & I 5 & ? & “ & 28 & 1866 & 2 & “ \\
\hline & I8 & ? & “ & 28 & I 866 & 2 & ، \\
\hline & 22 & ? & “ & 208 & I 865 & I & ، \\
\hline & 23 & Cedral & “ & 57 & I 866 & 2 & ، \\
\hline & 24 & Salinas & “ & 57 & I 866 & I \& 2 & ، \\
\hline & 25 & ? & “ & 57 & I 866 & 2 & • \\
\hline & 29 & ? & “ & 57 & I 866 & I & “ \\
\hline & 33 & Catorce & “" & 77 & I 866 & 2 & "، \\
\hline & 34 & Catorce & ، & 77 & I 866 & 2 & ، \\
\hline & 35 & Cedral & ، & 77 & I 866 & 2 & $: ،$ \\
\hline & 37 & ? & ، & 77 & I 866 & 2 & “ \\
\hline & 42 & Matehuala & ، & 97 & I 866 & $I \& 2$ & ، \\
\hline & 43 & Salinas & “ & 97 & I 866 & 2 & “ \\
\hline & $\begin{array}{l}43 \\
50\end{array}$ & Salinas & $" ،$ & I I I & I 866 & 2 & ، \\
\hline & 51 & Catorce & “ & 208 & 1865 & $1 / 2$ & “ \\
\hline & 53 & Matehuala & ، & 124 & I 866 & $I \& 2$ & “ \\
\hline & 54 & Catorce & ، & I 24 & I 866 & 2 & ، \\
\hline
\end{tabular}

* These may possibly be true Habilitados.

Series of Queretaro, (State of Queretaro.)

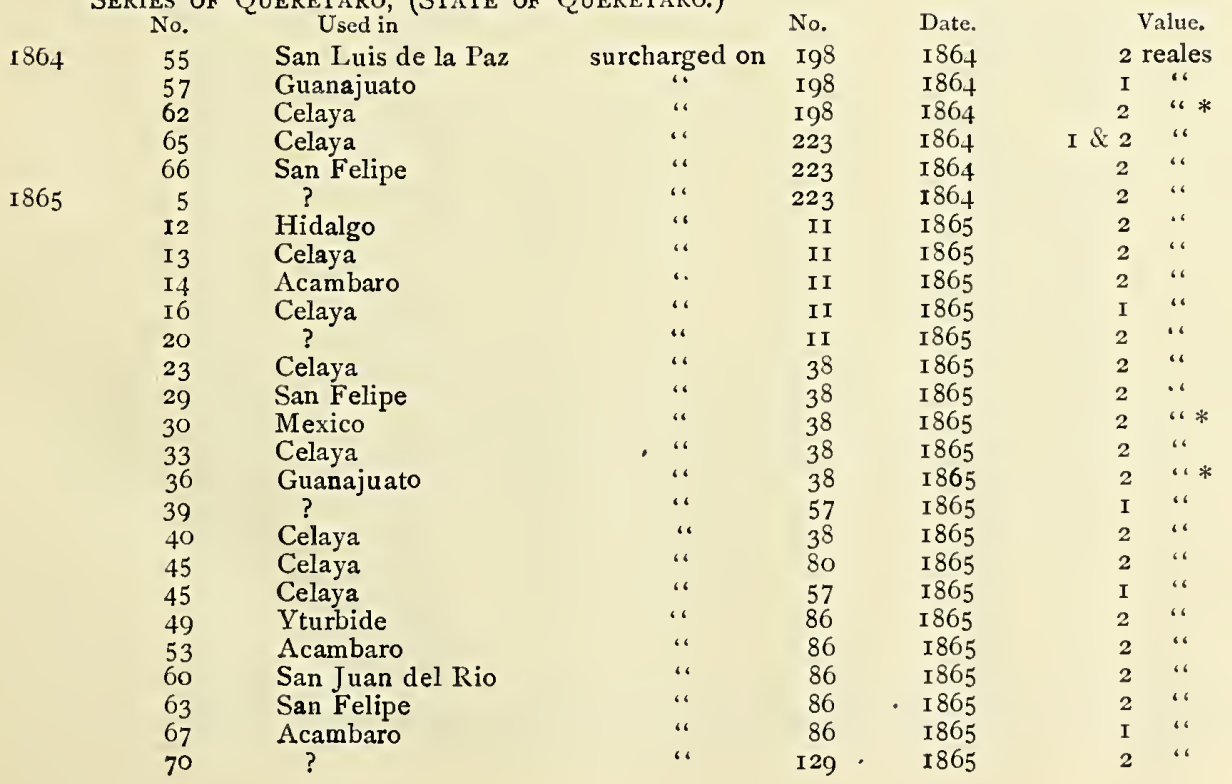


MEXICO.

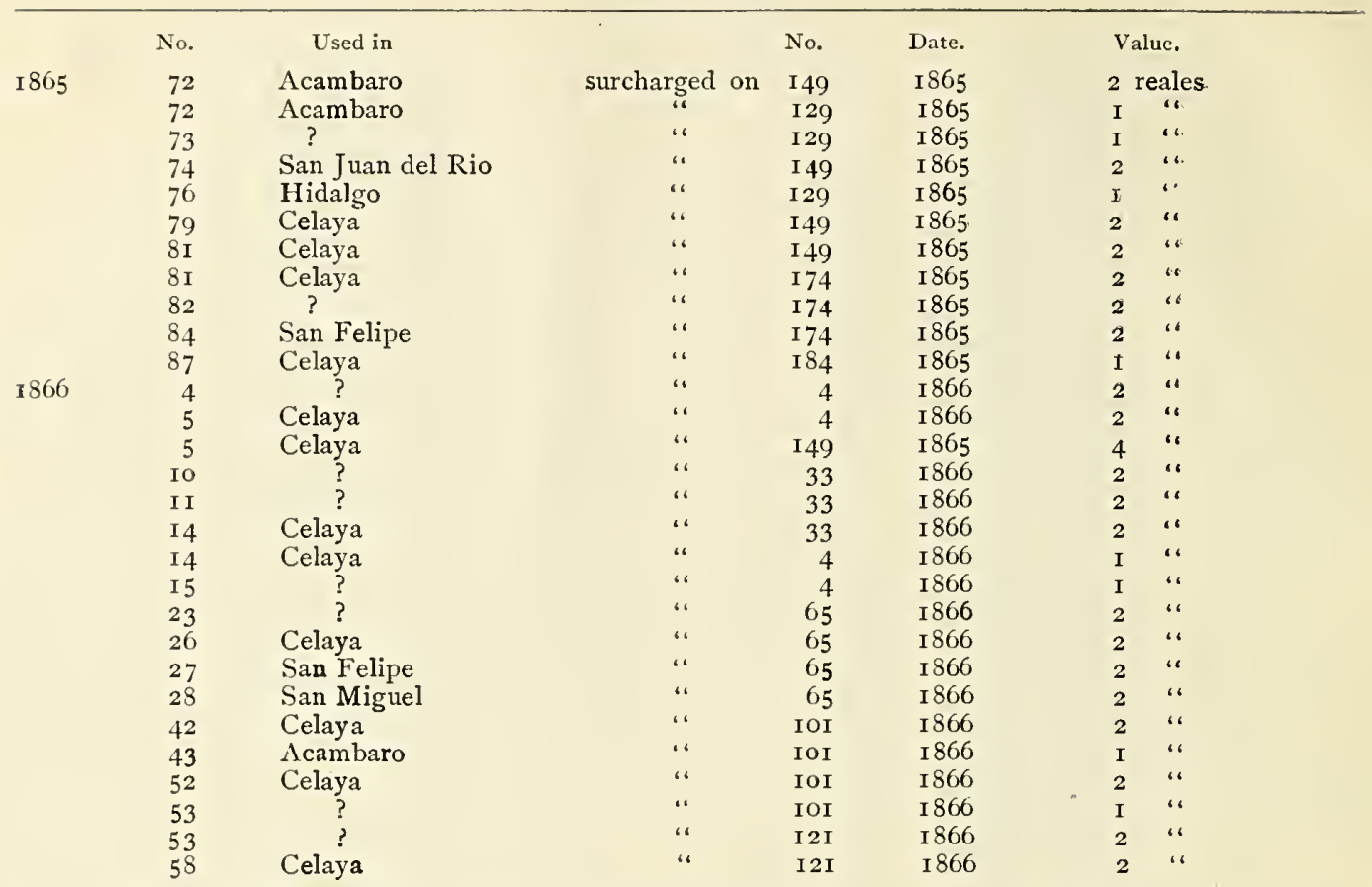

* Probably true Habilitados.

Celaya, Acambaro, San Luis de la Paz, San Felipe, San Miguel and Hidalgo are all in the state of Guanajuato. San Juan del Rio, is in the state of Hidalgo.

Series of Puebla (State of Puebla).

\begin{tabular}{|c|c|c|c|c|c|c|c|}
\hline \multirow{2}{*}{ I 864} & No. & Used iu & \multirow{3}{*}{ surcharged on } & No. & Date. & \multicolumn{2}{|c|}{ Value. } \\
\hline & 74 & Tehuacan & & I90 & I 864 & \multicolumn{2}{|c|}{2 reales } \\
\hline & 77 & Tehuacan & & Igo & I 864 & I & “' \\
\hline \multirow{26}{*}{ I 865} & 79 & 'Tehuacan & “ & 213 & I 864 & 2 & \\
\hline & I & Acatlan & ، & 240 & I 864 & 2 & "s, \\
\hline & 2 & ? & “ & 240 & I 864 & 2 & “. \\
\hline & I 5 & Tehuxcan & “ & 8 & I 865 & 2 & ، \\
\hline & $2 I$ & $?$ & " & 37 & I 865 & I & ، \\
\hline & 22 & Tehuacan & ، & 37 & I 865 & 2 & “ \\
\hline & 23 & $?$ & “ & 8 & I 865 & I & $\Leftrightarrow$ \\
\hline & 24 & ? & “ & 37 & I 865 & 2 & " \\
\hline & 28 & Acatlan & " & 37 & 1865 & 2 & $“$ \\
\hline & 33 & Tehuacan & " & 56 & I 865 & 2 & “ \\
\hline & $4 \mathrm{I}$ & Tehuacan & " & 87 & I 865 & 2 & 6 \\
\hline & 43 & $?$ & “ & 87 & I 865 & 2 & “ \\
\hline & 43 & $?$ & “ & 56 & I 865 & I & “ \\
\hline & 54 & Tehuacan & “ & I I 5 & I 865 & 2 & “ \\
\hline & 60 & Huamantla & “ & I I 5 & I 865 & 2 & “" \\
\hline & 65 & $?$ & “ & I I 5 & I 865 & 2 & “6 \\
\hline & 67 & Chalchicomula & ، & 87 & I 865 & I & “ \\
\hline & 68 & Tehuacan & " & I3 8 & I 865 & 2 & “ \\
\hline & 71 & Acatlan & ، & I38 & I 865 & 2 & “ \\
\hline & 77 & Huamantla & "، & 87 & I 865 & 4 & “ \\
\hline & 78 & ? & “ & I38 & I 865 & I & " \\
\hline & 84 & ? & “ & I56 & I 865 & 2 & “ \\
\hline & 87 & Tehuacan & ، & I 56 & I 865 & 2 & ، \\
\hline & $9 \mathrm{I}$ & $?$ & ، & I 90 & I 865 & 2 & 6 \\
\hline & 98 & ? & “ & I 56 & I 865 & I & ، \\
\hline & 99 & Tehuacan & " & I 90 & I 865 & 2 & " \\
\hline \multirow[t]{7}{*}{ I 866} & I I & Tehuacan & “ & 22 & I 666 & 2 & “ \\
\hline & I4 & ? & ، & 22 & I 866 & 2 & “ \\
\hline & I4 & $?$ & “ & 216 & I 865 & $I \& 2$ & “ \\
\hline & 24 & ? & “ & 53 & I 866 & 2 & “ \\
\hline & 38 & Tehuacan & “ & 53 & I 866 & 2 & “ \\
\hline & 43 & $?$ & “ & 53 & I 866 & 2 & “ \\
\hline & 53 & ? & “ & 53 & I 866 & I & 6 \\
\hline
\end{tabular}

IIuamantla is in the state of Tlaxcala, the other towns mentioned are in the state of Puebla, 

Series of Guanajuato (State of Guanajuato).

\begin{tabular}{|c|c|c|c|c|c|c|c|}
\hline \multirow{2}{*}{ I 864} & $\begin{array}{r}\text { No. } \\
77\end{array}$ & $\begin{array}{l}\text { Used in } \\
\text { Cancellation No. } 2\end{array}$ & \multirow{2}{*}{ surcharged on } & $\begin{array}{l}\text { No. } \\
2 O I\end{array}$ & $\begin{array}{l}\text { Date. } \\
1864\end{array}$ & \multicolumn{2}{|c|}{$\begin{array}{l}\text { Value. } \\
2 \text { reales }\end{array}$} \\
\hline & 78 & Cancellation No. I & & 217 & I 864 & I \& 2 & “ \\
\hline \multirow[t]{16}{*}{ I865 } & 6 & Cancellation No. I & •" & 7 & I 865 & 2 & :، \\
\hline & I9 & Cancellation No. I & “" & 7 & I 865 & 2 & “ \\
\hline & $3 I$ & Irapuato & “ & $5^{8}$ & $1865^{\circ}$ & 2 & “ \\
\hline & 36 & Cancellation No. I & “ & 58 & 1865 & 2 & “ \\
\hline & 37 & Leon & “، & 217 & I864 & 4 & " \\
\hline & $4 \mathrm{I}$ & Irapuato & “ & 58 & 1865 & 2 & “ \\
\hline & 44 & Cancellation No. I & “ & IOI & 1865 & 2 & "، \\
\hline & 47 & Cancellation No. I & “ & IOI & 1865 & 2 & “" \\
\hline & $5 \mathrm{I}$ & Cancella ion No. I & “. & 130 & 1865 & 2 & “" \\
\hline & 53 & Cancellation No. 2 & “" & 130 & 1865 & 2 & “، \\
\hline & 56 & Cancellation No. I. & “" & 130 & 1865 & 2 & “ \\
\hline & 60 & Irapuato & “" & I30 & 1865 & 2 & “ \\
\hline & $6 I$ & Cancellation No. I. & “" & -158 & 1865 & 2 & “، \\
\hline & 65 & Cancellation No. I. & “ & I 58 & 1865 & 2 & “" \\
\hline & 68 & Cancellation No. I. & “" & I 58 & 1865 & 2 & “ \\
\hline & $7 \mathrm{I}$ & Cancellation No. I. & “" & I 70 & 1865 & 2 & “ \\
\hline \multirow[t]{15}{*}{ I 866} & 13 & $?$ & “ & II & I $866^{\circ}$ & $1 / 2$ & “ \\
\hline & 14 & Leon & “" & II & I 866 & 2 & “ \\
\hline & I7 & Cancellation No. I. & “ & II & I 866 & I $\& 2$ & " \\
\hline & I9 & Leon & “" & 45 & I 866 & 2 & “" \\
\hline & 23 & Leon & “ & 45 & I 866 & 2 & “. \\
\hline & 26 & Cancellation No. I. & “" & 45 & I 866 & 2 & “" \\
\hline & 27 & Irapuato & “ & 60 & I 866 & 2 & “" \\
\hline & 29 & Leon & “ & 60 & I 866 & $1 \& 2$ & “" \\
\hline & 30 & Cancellation No. I. & “" & 60 & I 866 & 2 & “ \\
\hline & 32 & Leon & “" & 88 & I866 & 2 & “ \\
\hline & 34 & Leon & “ & 88 & I 866 & 2 & “ \\
\hline & 35 & Irapuato & “’ & 88 & I866 & 2 & “ \\
\hline & 38 & Leon & “ & 88 & I866 & I & “" \\
\hline & 38 & Leon & “ & 107 & I866 & 2 & “ \\
\hline & 40 & Irapuato & “ & 107 & I 866 & 2 & “" \\
\hline
\end{tabular}

Irapuato and Leon are in the State of Guanajuato.

Series of Durango (State of Durango).

The stamps of this series are always surcharged with name of issning district, Durango, and also with the extra date as well as the extra number. They are not common and the lowest number I have been able to find is No. 20, in 1865 , and I am rather inclined to doubt the various consignments being surcharged before that.

I 865

$\begin{array}{rl}\text { No. } & \text { Used in } \\ 20 & \text { Cuencame } \\ 22 & \text { Gavilanes } \\ 22 & \text { Gavilanes } \\ 24 & \text { Nazas } \\ 25 & \text { Papascuaro } \\ 29 & \text { Cuencame } \\ 30 & \text { Cuencame } \\ 34 & ? \\ * 35 & ? \\ 5 & \text { Cuencame } \\ * 7 & ? \\ 7 & ? \\ * \text { I5 } & ? \\ \text { I7 } & ? \\ \text { I0 } & \text { Cuencame } \\ \text { I0 } & \text { Cuencame } \\ \text { I6 } & \text { Cuencame } \\ \text { I6 } & \text { Cuencame } \\ 22 & \text { Cuencame }\end{array}$

\begin{tabular}{|c|c|}
\hline surcharged on & \\
\hline “ & \\
\hline “ & \\
\hline “" & \\
\hline "“ & \\
\hline " & \\
\hline " & \\
\hline " & \\
\hline “، & \\
\hline “، & \\
\hline “" & \\
\hline ، & \\
\hline " & \\
\hline “، & \\
\hline ، & \\
\hline " & \\
\hline
\end{tabular}

\begin{tabular}{|c|c|c|c|}
\hline Date. & \multicolumn{3}{|c|}{ Value. } \\
\hline $\begin{array}{l}1864 \\
\text { I } 864\end{array}$ & I & eal & \\
\hline 1865 & 2 & ، & \\
\hline 1865 & 2 & “" & \\
\hline I865 & I \& 2 & “. & \\
\hline I 865 & 2 & “، & \\
\hline $186^{\circ}$ & 2 & “" & \\
\hline I 865 & 2 & “" & \\
\hline 1865 & I & “" & (see note) \\
\hline I 866 & 2 & “، & \\
\hline 1865 & I & “) & \\
\hline I 866 & 2 & “" & \\
\hline 1865 & I & "\} & (see note) \\
\hline 1866 & 2 & “) & \\
\hline I 865 & I & "“ & \\
\hline I 866 & 2 & “" & \\
\hline I 865 & I & " & \\
\hline I 86 & I, $2, \& 4$ & “" & . \\
\hline I 866 & 2 & ، & \\
\hline
\end{tabular}

NOTE. - The above stamps marked with an asterisk were included in the series of Habilitados in the article for the Advanced Catalogue, but were merely included because of their being listed in Heitman's Handbuch. With the present increased knowledge of these stamps, it is now known that they were listed wrong, and that presumably their erroneous description must have been made from poor specimens. They have them $35-1865$ on I4, 7-I 866 on I4 and I6-I866 on $4 \mathrm{I}$, all of which it is now positive are wrongly described.

Series of Morelia (State of Michoacan).

\begin{tabular}{lrl} 
& No. & \multicolumn{1}{c}{ Used in } \\
& 965 & ? \\
& I 9 & Purnandiro \\
& 20 & Zamora \\
& 21 & Zamora \\
1866 & 35 & $?$ \\
& 4 & $?$ \\
& 5 & $?$
\end{tabular}

\begin{tabular}{|c|c|c|c|c|}
\hline surcharged on & $\begin{array}{l}\text { No. } \\
220\end{array}$ & $\begin{array}{l}\text { Date. } \\
\text { I } 864\end{array}$ & 4 & $\begin{array}{l}\text { Walue. } \\
\text { teales } \\
\text { "، }\end{array}$ \\
\hline 10 & 220 & I 864 & 2 & \\
\hline "6 & 220 & I 864 & 2 & “6 \\
\hline “ & 220 & I 864 & 2 & “6 \\
\hline “ & I 43 & I 865 & 2 & 6 \\
\hline " & 84 & I 865 & 8 & " \\
\hline " & 84 & I865 & 4 & f \\
\hline$"$ & тоб & I 866 & 2 & " \\
\hline
\end{tabular}


Series of Oaxaca (State of Oaxaca).

\begin{tabular}{|c|c|c|c|c|c|}
\hline $\begin{array}{r}\text { No. } \\
46\end{array}$ & $\begin{array}{l}\text { Used in } \\
\text { Huajaram }\end{array}$ & surcharged on & $\begin{array}{l}\text { No. } \\
108\end{array}$ & $\begin{array}{l}\text { Date. } \\
\text { I } 865\end{array}$ & $\begin{array}{l}\text { Value. } \\
2 \text { reales }\end{array}$ \\
\hline $5 \mathrm{I}$ & ? & " & 108 & 1865 & $1 / 2$ \\
\hline I 866 & ? & " & 42 & I 866 & ““ \\
\hline
\end{tabular}

Series of Pachuca (State of IIidalgo).

\begin{tabular}{|c|c|c|c|c|}
\hline $\begin{array}{r}\text { No. } \\
\text { I } 8\end{array}$ & $\begin{array}{c}\text { Used in } \\
\text { Zacualtipan }\end{array}$ & surcharged on & $\begin{array}{l}\text { No. } \\
210\end{array}$ & $\begin{array}{c}\text { Date. } \\
\text { I } 864\end{array}$ \\
\hline 29 & Chico & & I 24 & I $865^{\circ}$ \\
\hline
\end{tabular}

Series of Monterrey (State of Nuevo Leon).

No. Used in $\quad$ surcharged on
$20 \quad$ ?

$\begin{array}{lll}\text { No. } & \text { Date. } & \text { Value. } \\ 212 & \text { I } 867 & 2 \text { reales }\end{array}$

Series of Tulancingo (State of Hidalgo).

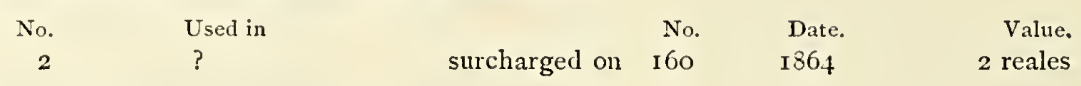

Series of Cordova (State of Vera Cruz).

\begin{tabular}{|c|c|c|c|c|c|c|}
\hline & No. & Used in & & No, & Date. & Value. \\
\hline & I3 & ? & surciarge on & 144 & I 865 & I \\
\hline I 866 & I 2 & Vera Cruz & “" & $4 \mathrm{I}$ & I $866^{\circ}$ & 2 \\
\hline
\end{tabular}

Series of Toluca (State of Mexico).

\begin{tabular}{|c|c|c|c|c|c|c|}
\hline No. & Date. & Used in & & No. & Date. & Value. \\
\hline I4 & I 865 & ? & surcharged on & $9^{I}$ & I 865 & 2 reales \\
\hline I6 & 1865 & Mexico & $\therefore$ & 47 & I 865 & I \\
\hline I 6 & I 865 & Mexico & “" & $9 \mathrm{I}$ & 1865 & 2 \\
\hline$\star_{27}$ & I 865 & ? & “. & I02 & I 865 & “. \\
\hline 15 & I 866 & ? & “" & 79 & 1866 & 2 reales \\
\hline
\end{tabular}

*This is thus described in Heitman's Handbuch but was probably copied by them from Lockyer's list, and is without question an error, "IO2-I865" having been the regular consignment to Zacatecas. As yet we have been unable to find No. 27-1865 to enable us to correct the mistake.

August, 1866.

Lithographed (portrait of Maximilian) on white wove paper. Size $19 \times 22 \mathrm{~mm}$.

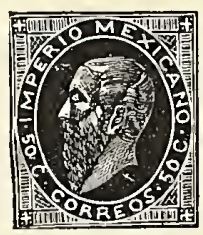

I, Without surcharge.

$7 \mathrm{c}$ lilac

$7 \mathrm{c}$ gray lilac

$7 \mathrm{c}$ deep lilac

I $3 \mathrm{c}$ blue

I $3 c$ light blue

I $3 \mathrm{c}$ deep blue

$25 \mathrm{c}$ yellow (shades)

$25 \mathrm{c}$ orange

$25 \mathrm{c}$ deep reddish orange

5 oc green

$50 \mathrm{c}$ pale green

5 oc dark green

$50 \mathrm{C}$ yellow green

II. Surcharged with name of postal dis-

trict only.

$763 \quad 7 \mathrm{c}$ lilac

$7647 \mathrm{c}$ gray lilac

$7 \mathrm{c}$ deep lilac

I $3 \mathrm{c}$ blue

767 I3c light blue
768 I $3 \mathrm{c}$ deep blue

$769 \quad 25 \mathrm{c}$ yellow (shades)

$77025 \mathrm{c}$ orange "

$77 \mathrm{I} 25 \mathrm{c}$ deep reddish orange

$772 \quad 50 c$ green

773 50c pale green

7745 oc dark green

775 50c yellow green

SURCHARgES FOUND ON THIS ISSUE:

\begin{tabular}{|c|c|}
\hline District, & Meas, in $\mathrm{mm}$. \\
\hline CORDOVA & I $5 \times 21 / 2$ \\
\hline CUERNAVACA & $181 / 2 \times 13 / 4$ \\
\hline 理 & $5 \times 2 ; M 2 I$ \\
\hline PUEBLA & I $31 / 2 \times 21 / 4$ \\
\hline TAMPICO & $15 \times 2$ \\
\hline
\end{tabular}

III. Surcharged with name of postal district and date $\mathbf{I} 866$.

$776 \quad 7 \mathrm{c}$ lilac

$777 \quad 7 \mathrm{c}$ gray lilac

$778 \quad 7 \mathrm{c}$ deep lilac

779 I 3c blue

780 I $3 \mathrm{c}$ light blue

$78 \mathrm{I}$ I3c deep blue

$782 \quad 25 \mathrm{C}$ yellow (shades)

$783 \quad 25 \mathrm{c}$ orange

$784 \quad 25 \mathrm{c}$ deep reddish orange

785 50c green

$78650 \mathrm{Oc}$ pale green

$78750 \mathrm{c}$ dark green

788 50c yellow green 

Variety: Blue dot surrounded by small double white circle instead of white dot behind the value (I3c).

$789 \quad$ r 3 c dark blue

IV. Surcharged with name of postal district and date 866

$790 \quad 7 \mathrm{c} \mathrm{lilac}$

$79 I \quad 7 c$ gray lilac

$792 \quad 7 \mathrm{c}$ deep lilac

793 r $3 \mathrm{c}$ blue

794 I3c light blue

795 I3c deep blue

$796 \quad 25 \mathrm{c}$ yellow (shades)

$79725 \mathrm{c}$ orange

79825 c deep reddish orange

$79950 c$ green

$800 \quad 50 \mathrm{c}$ pale green $\begin{array}{ll}801 & 50 c \text { dark green } \\ 802 & 50 c \text { yellow green }\end{array}$

V. Surcharged with number and date only.

$803 \quad 7 \mathrm{c}$ lilac

$804 \quad 7 c$ gray lilac

$8057 \mathrm{c}$ deep lilac

$806 \quad 13 \mathrm{c}$ blue

807 r3c light blue

808 r3c deep blue

$809 \quad 25 \mathrm{c}$ yellow (shades)

$81025 \mathrm{c}$ orange

8 II $25 \mathrm{C}$ deep reddish orange

$8 \mathrm{I} 250 \mathrm{C}$ green.

8I 3 5Oc pale green

$81450 c$ dark green

815 5oc yellow green

List of Surcharges (Name of District, Number and Date) found on Stamps of this Issue.

\begin{tabular}{|c|c|}
\hline District. & feas. in $\mathrm{mm}$. \\
\hline ACUASCALIENT & $251 / 2 \times 3$ \\
\hline APAM & $91 / 2 \times 2$ \\
\hline COCULA & $12 \times 2$ \\
\hline CORDOVA & $15 \times 21 / 2$ \\
\hline .CORDOVA. & I $6 \times 21 / 2 ;$ bet. periods \\
\hline CUERNAVACA & $181 / 2 \times 13 / 4$ \\
\hline CHALCO & $13 \times 2$ \\
\hline DURANGO & $\mathrm{x} 5 \times 21 / 4$ \\
\hline GUAD A LAJARA & $20 \times 2$ \\
\hline GUANAJUATO & $2 I 1 / 2 \times 3$ \\
\hline GUANAJUATO & $17 \times 21 / 2$ \\
\hline IXTLAHUACA & I $7 \times 1 \mathrm{I} / 2$ \\
\hline JALAPA & $\operatorname{IIXI} 3 / 4$ \\
\hline LAGOS & I I $1 / 2 \times 2$ \\
\hline LAGOS & $\operatorname{IOx} 2$ \\
\hline MARAVATIO & $17 \times 2$ \\
\hline MERIDA & $13 \times 2$ \\
\hline & I $3 \times 1 / 2$ to 3 \\
\hline ERIDA & I $3 \times 1 / 2$ \\
\hline
\end{tabular}

\begin{tabular}{|c|c|}
\hline $\begin{array}{c}\text { District. } \\
\text { SoxeXxico }\end{array}$ & $\begin{array}{l}\text { Meas, in } \mathrm{mm} . \\
\quad 15 \times 2 ; \mathrm{M}_{2} 1 / 2\end{array}$ \\
\hline Morelia & I6 $1 / 2 \times 13 / 4 ; M$ and $121 / 2$ \\
\hline - MORELIA. & I $41 / 2 \times 2$; bet. periods \\
\hline UAXACA & $141 / 2 \times 21 / 4$ \\
\hline ORIZAVA. & I $71 / 2 \times 2$; with period \\
\hline PACHUCA & $15 \times 21 / 2$ \\
\hline PUEBLA. & I3 $3 / 4 \times 23 / 4 ;$ with period \\
\hline QUERETARO & $171 / 2 \times 2$ \\
\hline S.L.POTOSI. & I $7 \times 3$; with period \\
\hline SOYANIQUILPAM & $20 \times 2$ \\
\hline TAM PICO & $15 \times 2$ \\
\hline TEP I J & $12 \times 2$ \\
\hline TEPIC & $8 \times 1 / 2$ \\
\hline $\begin{array}{l}\text { TOLUCA } \\
\text { TULA }\end{array}$ & $\begin{array}{l}\text { I } 2 \times 2 \\
8 \times 2\end{array}$ \\
\hline TULANCINCO & $18 \times 2$ \\
\hline VERACRIJ\% & $131 / 2 \times 13 / 4$ \\
\hline VERACRUZ. & I9x2; with period \\
\hline YGUALA & I $31 / 2 \times 2$ \\
\hline ZAPOTLAN & $14 \times 2$ \\
\hline
\end{tabular}

List of the office numbers occurring on these stamps.

\begin{tabular}{|c|c|c|c|c|c|c|c|}
\hline I & Mexico & 29 & Tula & 56 & Aguascalientes. & & Cuernavaca \\
\hline 2 & Mexico & 30 & Yguala & 57 & $?$ & 83 & Guanajuato \\
\hline 3 & Puebla & $3 I$ & ? & 58 & S. L. Potosi & 84 & ? \\
\hline 4 & Guanajuato & 32 & Pachuca & 59 & Chalco & 85 & Veracruz \\
\hline 5 & Veracruz & 33 & Queretaro & 60 & ? & 86 & ? \\
\hline 6 & Queretaro & 34 & Ápam & $6 I$ & Veracruz & 87 & Mexico \\
\hline 7 & Orizava & 35 & Oaxaca & 62 & Orizava & 88 & Cuernavaca \\
\hline 8 & Cordova & 36 & Cuernavaca & & Cuernavaca & 89 & Chalco \\
\hline 9 & Ixtlahuaca & 37 & Mexico & 63 & Veracruz & 90 & ? \\
\hline IO & Mexico & 38 & Mexico & 64 & ? & 9I & Puebla \\
\hline I I & Cuernavaca & 39 & Veracruz & 65 & ? & & Guanajuato \\
\hline I 2 & S. L. Potosi & 40 & Orizava & 66 & Mexico & 92 & Mexico \\
\hline I3 & Toluca & $4 I$ & Cocula & 67 & $?$ & 93 & Mexico \\
\hline I4 & Soyaniquilpam & & Guadalajara & 68 & Zacatecas & 94 & S. L. Potosi \\
\hline I5 & Tepeji & & Tepic & 69 & Chalco & 95 & Mexico \\
\hline I6 & Morelia & 42 & $?$ & 70 & Mexico & 96 & Lagos \\
\hline I7 & Maravatio & 43 & Mexico & $7 I$ & S. I. Potosi & 97 & Cuernavaca \\
\hline 18 & Durango & 44 & Zapotlan & & Colima & 98 & Saltillo \\
\hline I9 & Zacatecas & 45 & Cordova & 73 & S. L. Potosi & 99 & Mexico \\
\hline 20 & Apam & 46 & Puebla & 74 & S. L. Potosi & IOO & Mexico \\
\hline $2 \mathrm{I}$ & Tulancingo & 47 & Guanajuato & 75 & Guadalajara & IOI & Mexico \\
\hline 22 & Lagos & 48 & ? & 76 & Toluca & 102 & Zacatecas \\
\hline 23 & Mexico & 49 & Queretaro & 77 & Queretaro & 103 & Quere'aro \\
\hline 23 & Chalco & 50 & Morelia & 78 & Ãpam & 104 & Mexico \\
\hline 24 & Guanajuato & $5 \mathrm{I}$ & Jalapa & 79 & Morelia & J06 & Orizava \\
\hline 25 & $?$ & 52 & Merida & 80 & Mexico & 107 & Mexico \\
\hline 26 & $?$ & 53 & Veracruz & $8 I$ & $?$ & 108 & also ros 866 \\
\hline 27 & Mexico & 54 & ? & 82 & Puebla & & Puebla \\
\hline
\end{tabular}




\begin{tabular}{ccccccc}
\hline IO9 Guadalajara & Ir4 also Ir4 866, & I20 & Mexico & I30 Mexico \\
IIO also IIo 866, & Mexico & I27 & Mexica & I39 Mexica \\
Merida & & &
\end{tabular}

ERRORS.

With number and date inverted.

I I866 Mexico on 25c

6 r866 Queretaro on I3c

33 r866 Queretaro on 7c

With one of the figures above the line.

I ro 86 (Merida) on $7 c$

With one or more of the figures missing

$\begin{array}{lll}78 & \text { I } & \text { (Apam) on } 25 \mathrm{C} \\ 78 & \text { I8 } & \text { (Apam) on } 25 \mathrm{C} \\ 78 & 186 & \text { (Apam) on } 25 \mathrm{C}\end{array}$

Sub-Districts,

Series of Guadalajara (State of Jalisco).

$\begin{array}{rc}\text { No. } & \text { Used in } \\ 20 & \text { Cocula } \\ 27 & ? \\ 27 & ? \\ 33 & \text { Tepic } \\ 35 & \text { Celaya }\end{array}$

$\begin{array}{ccc} & \text { No. } & \text { Date. } \\ \text { surcharged on } & 4 \mathrm{I} & \mathrm{I} 866 \\ \because . & 4 \mathrm{I} & \mathrm{I} 866 \\ \text { “. } & 4 \mathrm{I} & \mathrm{I} 866 \\ \text { “ } & 4 \mathrm{I} & \mathrm{I} 866 \\ & 75 & \mathrm{I} 866\end{array}$

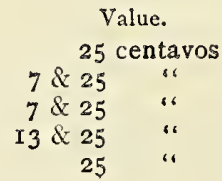

Series of Jalapa (State of Vera Cruz)

No.

I7

Date. Used in
r866 ?

Series of Zacatecas (State of Zacatecas).

No.
56
57
58
70
72
77

Series of Queretaro (State of Queretaro).
No.

I

II

I5

I9

25
28

32

* Possibly a true Habilitado.

Series of Puebla (State of Puebla).

Used in
Acatlan
$?$
$?$
Chalchicomula

No.

surcharged on

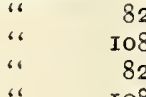

No.

surcharged on $5 \mathrm{I}$
Date.

I866 $7,13,25,50$ centavos

\begin{tabular}{|c|c|c|c|c|c|}
\hline \multirow{4}{*}{$\begin{array}{l}\text { Used in } \\
\text { Aguascallientes } \\
\text { (Aguascaltes) }\end{array}$} & \multicolumn{2}{|r|}{ No. } & \multirow{3}{*}{$\begin{array}{l}\text { Date. } \\
\text { r } 866 \\
\text { r } 866\end{array}$} & \multicolumn{2}{|c|}{ Value. } \\
\hline & surcharged on & r9 & & & entav \\
\hline & & I9 & & $13 \& 2$ & "“ \\
\hline & " & I9 & 1866 & 2 & "“ \\
\hline Aguascallientes & " & 68 & r866 & 2 & “" \\
\hline ? & " & 68 & 1866 & 2 & “ \\
\hline (Aguascaltes) & "“ & $\mathrm{IO} 2$ & I 866 & 25 & "“ \\
\hline
\end{tabular}

Series of Guanajuato (State of Guanajuato).
No.

Series of Morelia (State of Michoacan).

$$
\text { Used in }
$$

Leon

Irapuato

Cancellation No. 2

Leon

Leon

Cancellation No, 2

Leon

$\begin{array}{cr} & \text { No. } \\ \text { surcharged on } & 4 \\ \text { "، } & 4 \\ \text { “ } & 47 \\ \text { " } & 47\end{array}$

Date.

I 866

I 866

I 866

1866

1866

I 866

I 866

Value. 25 centavos

I3 "

25

25
No.

$$
\begin{gathered}
\text { Used in } \\
? \\
?
\end{gathered}
$$

surcharged on $\begin{array}{r}\text { No } \\ \text { I } 6\end{array}$
Date.

I 866

I 866
Value, I3 centavos 




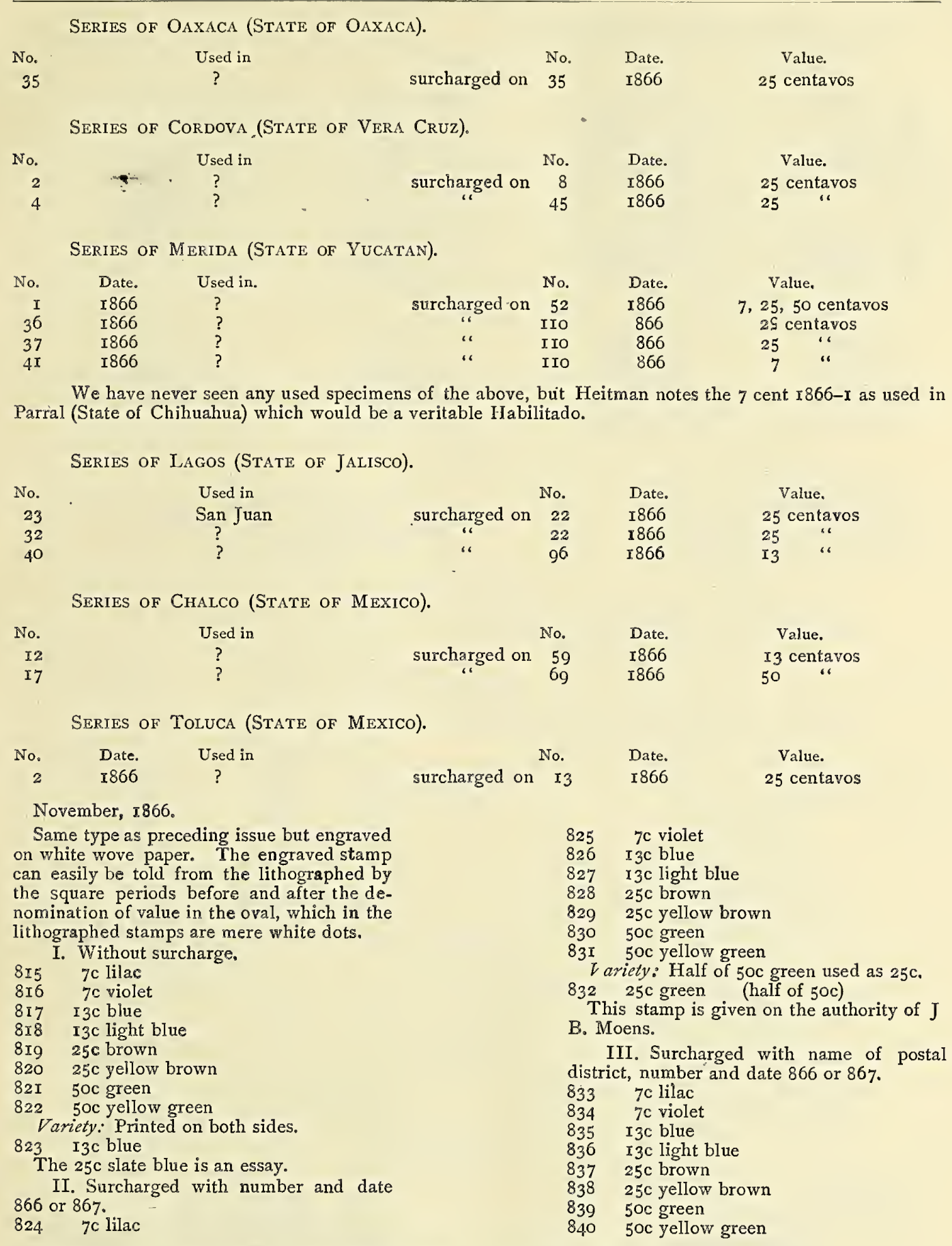

List of surcharges (names of postal districts) found on stamps of this issue.

\begin{tabular}{|c|c|c|c|}
\hline A PAM & $\mathrm{I} 2 \times 2 \mathrm{I} / 4$. & IVorelia & $\mathrm{I} 6 \mathrm{I} / 2 \mathrm{xI} 3 / 4, \mathrm{M}$ and $\mathrm{I} 2 \mathrm{I} / 2$. \\
\hline - CORDOVA. & $\begin{array}{l}\text { I } 5 \times 2 \text {, between two } \\
\text { periods. }\end{array}$ & O R I ZAVA. & $171 / 2 \times 2$, with period. \\
\hline $\begin{array}{l}\text { CORDOVA } \\
\text { CHALCO }\end{array}$ & $15 \times 21 / 2$ & PUEBLA. & $\begin{array}{l}17 / 2 \text {, with period. } \\
131 / 2 \times 21 / 2 \text {, with period. }\end{array}$ \\
\hline GUADALAJARA & $20 \times 2$ & QUERETARO & $\mathrm{I} 7 \mathrm{I} / 2 \times 2$ \\
\hline GUANAJUATO & $17 \times 21 / 2$. & TEPIC & $71 / 4 \times 2$ \\
\hline GUANAJUATO & $\mathrm{I} 7 \times 2 \mathrm{I} / 2$. & TOLUCA & $12 \times 2$. \\
\hline JALAPA & $\operatorname{IIXI} 3 / 4$. & TULA & $8 \times 2$ \\
\hline MERIDA & $13 \times 2$. & VERACRUZ & $131 / 2 \times 13 / 4$. \\
\hline soxexico & $5 \times 2, M 2 \frac{1}{2}$ & YGUALA & $12 \times 2$. \\
\hline
\end{tabular}


List of post office numbers found on this issue.

866

$\begin{array}{ll}\text { IOI } & \text { Veracruz } \\ \text { IO2 } & \\ \text { I03 } & \text { Queretaro } \\ \text { I04 } & \text { Mexico } \\ \text { I05 } & \\ \text { I06 } & \\ \text { I07 } & \\ \text { I08 } & \text { Puebla } \\ \text { I09 } & \text { Guadalajara } \\ \text { I I0 } & \text { Merida }\end{array}$

$\begin{array}{ll}\text { II I } & \text { Mexico } \\ \text { II2 } & \text { Guanajuato } \\ \text { II3 } & \text { Tula } \\ \text { I 14 } & \text { Mexico } \\ \text { II5 } & \text { Veracruz } \\ \text { II6 } & \\ \text { II7 } & \\ \text { II8 } & \text { Yguala } \\ \text { II9 } & \text { Mexico } \\ \text { I20 } & \text { Mexico }\end{array}$

$\begin{array}{ll}\text { I } & \text { Mexico } \\ 2 & \text { Orizava } \\ 3 & \text { Mexico } \\ 4 & \\ 5 & \text { Cordova }\end{array}$

6 Mexico

7 Puebla

8 Mexico

IO Mexico

$\begin{array}{ll}\text { I21 } & \text { Morelia } \\ \text { I22 } & \\ \text { I23 } & \text { Puebla } \\ \text { I24 } & \text { Orizava } \\ \text { I25 } & \text { Toluca } \\ \text { I26 } & \\ \text { I27 } & \text { Mexico } \\ \text { I28 } & \text { Veracruz } \\ \text { I29 } & \text { Chalco } \\ \text { I30 } & \text { Mexico }\end{array}$

$\begin{array}{ll}\text { I31 } & \\ \text { I32 } & \\ \text { I33 } & \text { Puebla } \\ \text { I34 } & \\ \text { I35 } & \text { Mexico } \\ \text { I36 } & \text { Mexico } \\ \text { I37 } & \text { Queretaro } \\ \text { I38 } & \text { Cordova } \\ \text { I39 } & \text { Mexico }\end{array}$

867

$\begin{array}{llll}\text { I } & \text { Mexico } & \text { I6 } & \text { Apam } \\ \text { I2 } & \text { Mexico } & \text { I7 } & \\ \text { 13 } & \text { Mexico } & \text { I8 } & \text { Mexico } \\ \text { 14 } & \text { Mexico } & \text { I9 } & \text { Mexico } \\ \text { I5 } & \text { Mexico } & & \end{array}$

Name inverted.

I37 I866 Queretaro on I3c
I37 I 866 on on 25c
Number and date inverted.

ERrors :

III I 866 Mexico on $25 \mathrm{c}$
Name, number and date inverted.
I 5 I 866 Vera Cruz on $25 \mathrm{c}$

SUR-DisTRICTS.

Series of Guadalajara (State of Jalisco).

$\begin{array}{cccccc} & \text { No. } & \text { Used in } & \text { No. } & \text { Date. } & \text { Value. } \\ \mathbf{1} 866 & 37 & ? & \text { surcharged on rog } & 866 & 25 \text { centavos }\end{array}$

Series of Queretaro (State of Queretaro).

\begin{tabular}{|c|c|c|c|c|c|c|}
\hline \multirow{3}{*}{ I 866} & No. & Used in & & No. & Date. & Value. \\
\hline & 43 & ? & surcharged or & 137 & 866 & 25 centavos \\
\hline & 46 & ? & $\therefore$ & 137 & 866 & $I_{3} \& 25 \cdots$ \\
\hline \multirow[t]{2}{*}{1867} & 2 & ? & “" & I37 & 896 & 25 \\
\hline & 4 & ? & “. & 137 & 866 & I3\& 25 \\
\hline
\end{tabular}

Series of Puebla (State of Puebla).

$\begin{array}{lccccc} & \text { No. } & \text { Used in } & \text { No. } & \text { Date. } & \text { Value. } \\ \mathbf{1} 866 & \mathbf{5} & ? & \text { surcharged on } \mathbf{1 0} & 866 & \text { I3 centavos }\end{array}$

Series of Cordova (State of Vera Cruz).

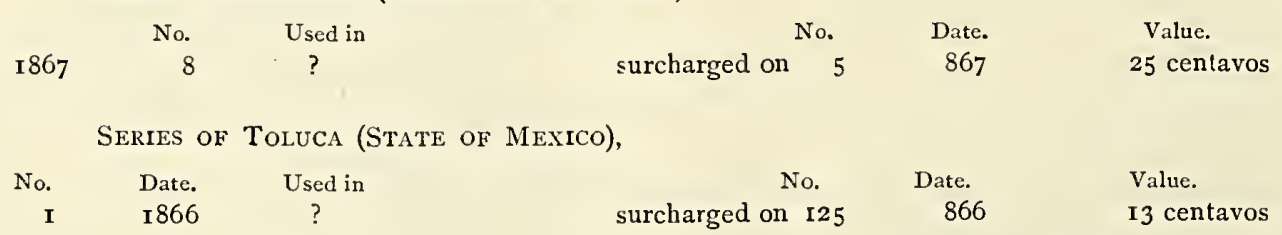

June, r867.

Provisional issue.

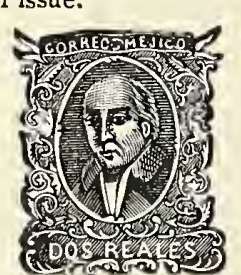

I. Stamps of the issues of August, I 865 , and January and November, I86r, surcharged

SHeXXic o in Gothic type in black.

84 I $1 / 2$ (Medio) r black on brown

842 I (un) $r$ black on green

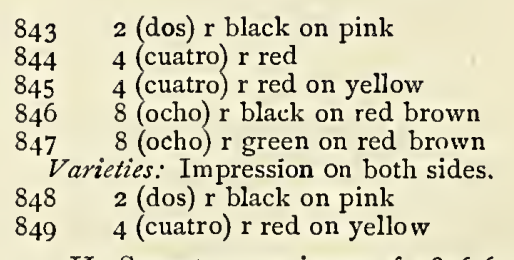

II. Same type as issues of $1856-6 \mathbf{I}$, but printed on bluish wove paper, watermarked R. P.S. (Renta Papel Sellado) in the sheet. A certain number of the stamps show only a part of the watermark.

Io Without any surcharge.

$\begin{array}{ll}850 & 1 / 2 \text { (Medin) r gray } \\ 851 & \text { I (un) r blue } \\ 852 & 2 \text { (dos) r green } \\ 853 & 4 \text { (cuatro) r pink }\end{array}$




\section{Reprints.}

I 8 ?

Thick wove paper.

$854 \quad 1 / 2$ (Medio) r gray

8558 (ocho) r gray

These two stamps are also found surcharged Mexico in Gothic type, which surcharge, however, is forged and of which

we give a fac-simile here atrex so type.

$2^{\circ}$ Surcharged Sflexico in Gothic

$856 \quad 1 / 2$ (Medio) $r$ gray

857 I (un) $r$ blue

8582 (dos) $r$ green

8594 (cuatro) $r$ pink

Varieties:

a. Double surcharge.

$8602($ dos $) r$ green

b. I real stamps cut in two, each half being used as $I$ real.

86 I $1 / 2 \mathrm{r}$ blue (half of $\mathrm{I}$ real).

August, I 868.

Bust of Hidalgo lithographed on colored wove paper; there are two different platesplate I and II-of all values, both made from one original die, the figures representing the denomination of value being added separately on each of the 200 stamps composing each plate, thus forming 200 minor varieties of each value and plate. The difference between plates I and II consists in the figures of value, which in the Ist plate are long and thin, and not followed by a period, while in plate II they are shorter and thicker and are followed by a period. Size $171 / 2 \times 231 / 2$ $\mathrm{mm}$.

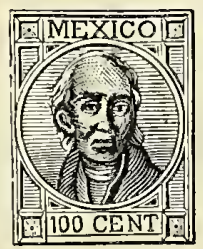

Plate I.

\section{CENT. 12 CENT. 25 CENT. 50 CENT, 100 CENT}

Plate II.

\section{CENT. 12. CENT. 25. CENT. 50 CENT. 100.CENT.}

Rounded saw tooth perforation. nunurunuuruuruurum

Pointed saw tooth perforation. nиssunsunusnum

Square perforation.
Pin perforation.

Regular perforation.

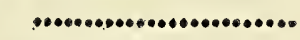

I. Without surcharge.

$I^{\circ}$ Unperforated.

Printed on ordinary colored wove paper varying but little in thickness.

862 6c black on light brown

863 I $2 \mathrm{c}$ black on yellow green

864 I2c black on sea green

$86525 \mathrm{c}$ blue on light pink

866 25c bluish black on light pink

867 50c black on yellow

868 IOoc black on reddish brown

Varieties:

a. Error of color

869 IOOc brown on brown

b. Period after I2.

870 I 2c black on yellow green

87 I I2c black on sea green

$2^{\circ}$ Rounded saw tooth perforation.

$8726 \mathrm{c}$ black on light brown

873 I 2c black on yellow green

874 I2c black on sea green

$87525 \mathrm{c}$ blue on light pink

$876 \quad 25 \mathrm{c}$ bluish black on light pink

877 50c black on yellow

878 Iooc black on reddish brown

Varieties.

a. Error of color.

879 Iooc brown on brown

b. Period after I 2 .

880 I $2 \mathrm{c}$ black on yellow green

88I I 2c black on sea green

II. Surcharged with number and abbreviated date on right hand side of stamp.

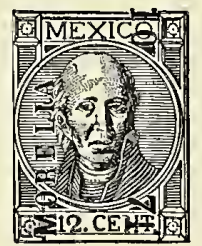

$I^{\circ}$ Unperforated.

$6 \mathrm{c}$ black on light brown

I $2 \mathrm{c}$ black on yellow green

I 2 c black on sea green

$25 \mathrm{c}$ blue on light pink

$25 \mathrm{c}$ bluish black on light pink

5 oc black on yellow

887 50c black on yellow

888 rooc black

a. Error of color.

889 rooc brown on brown

b. IOoc stamps divided in fourths, each

fourth being used as a $25 \mathrm{c}$ stamp.

$890 \quad 25 \mathrm{c}$ black on brown (upper right fourth of $\mathrm{IOOC}$ )

$89 \mathrm{I} 25 \mathrm{c}$ black on brown (lower right fourth of 100 )

$89225 \mathrm{c}$ black on brown (upper left fourth of rooc)

$89325 \mathrm{c}$ black on brown (lower left fourth of 100 )

c. 5oc stamps cut vertically in two, each half being used as a $25 \mathrm{c} \mathrm{slamp}$.

$89425 \mathrm{c}$ black on yellow (right half of $5 \mathrm{Oc}$ )

895 25c black on yellew (left half of 50c)

d. 5oc stamps cut horizontally in two, each

half being used as a $25 \mathrm{c}$ stamp.

$89625 \mathrm{c}$ black on yellow (upper half of $50 \mathrm{c}$ )

$89725 \mathrm{c}$ black on yellow (lower half of $50 \mathrm{c}$ ) 
e. 50c stamps cut diagonally in two, each half being used as a $25 \mathrm{c}$ stamp.

$89825 \mathrm{c}$ black on yellow (upper right half of $50 \mathrm{Oc})$

$89925 \mathrm{c}$ black on yellow (lower right half of $50 \mathrm{Oc}$ )

$90025 \mathrm{c}$ black on yellow (upper left half of 5Oc)

90I $25 \mathrm{c}$ black on yellow (lower left half of $50 c$ )

f. Period after I2.

902 I $2 \mathrm{c}$ black on yellow green

903 I2c black on sea green

$2^{\circ}$ Rounded saw tooth perforation.

$9046 \mathrm{c}$ black on light brown

905 I $2 \mathrm{c}$ black on yellow green

906 I 2c black on sea green

$90725 \mathrm{c}$ blue on light pink

$908 \quad 25 \mathrm{c}$ bluish black on light pink

$90950 \mathrm{c}$ black on yellow

9IO Iooc black on reddish brown

Varieties:

Error of color.

9II IOOc brown on brown

b. tooc stamps divided in fourths, each fourth being used as a $25 \mathrm{c}$ stamp.

$91225 \mathrm{c}$ black on brown (upper right $1 / 4$ of IOOC)

9 $1325 \mathrm{c}$ black on brown (lower right $1 / 4$ of IOOC)

9 I4 $25 \mathrm{c} \mathrm{black}$ on brown (upper left $1 / 4$ of IOOC)

9 I5 $25 \mathrm{c}$ black on brown (lower left $1 / 4$ of IOOC)

c. 5oc stamps cut vertically in two, each half being used as a $25 \mathrm{c}$ stamp.

$91625 \mathrm{c}$ black on yellow (right half of $50 \mathrm{c}$ )

9I $725 \mathrm{c}$ black on yellow (left half of $5 \mathrm{Oc}$ )

d. 5oc stamps cut horizontally in two, each

half being used as a $25 \mathrm{c}$ stamp.

$918 \quad 25 \mathrm{c}$ black on yellow (upper half of $50 \mathrm{c}$ )

9 I9 $25 \mathrm{c}$ black on yellow (lower half of $5 \mathrm{Oc}$ )

e. 5 oc stamps cut diagonally in two, each half being used as a $25 \mathrm{c}$ stamp.

$92025 \mathrm{c}$ black on yellow (upper right half of $50 \mathrm{Oc}$ )

92 I $25 \mathrm{c}$ black on yellow (lower right half of 50c)

$92225 \mathrm{c}$ black on yellow (upper left half of 50c)

$92325 \mathrm{c}$ black on yellow (lower left half of

f. Poc)

924 I 2 c black on yellow green

925 I $2 \mathrm{c}$ black on sea green

III. Surcharged with number and abbreviated date on right hand side of stamp with the addition of the name of the postal district.

I $^{\circ}$ Unperforated.

926. $6 \mathrm{c}$ black on light brown

927 I $2 \mathrm{c}$ black on yellow green

928 I $2 \mathrm{c}$ black on sea green

$92925 \mathrm{c}$ blue on light pink

$930 \quad 25 \mathrm{c}$ bluish black on light pink

93 I $50 \mathrm{c}$ black on yellow

932 Iooc black on red brown

Varieties.

a. Error of color.

933 IOoc brown on brown

b. IOOc stamps divided in fourths, each fourth being used as a $25 \mathrm{c}$ stamp.

$934 \quad 25 \mathrm{c} \mathrm{black}$ on brown (upper right fourth of IOOC)

$93525 \mathrm{c}$ black on brown (lower right fourth of $100 c$ )

$93625 \mathrm{c}$ black on brown (upper left fourth of IOOC)
$93725 \mathrm{c}$ black on brown (lower left fourth of 1000 )

c. 5oc stamps cut vertically in two, each half being used as a $25 \mathrm{c}$ stamp.

$938 \quad 25 \mathrm{c}$ black on yellow (right half of $50 \mathrm{c}$ )

$93925 \mathrm{c}$ black on yellow (left half of $50 \mathrm{c}$ )

d. 5oc stamps cut horizontally in two, each half being used as a $25 \mathrm{c}$ stamp.

$94025 \mathrm{c}$ black on yellow (upper half of $50 \mathrm{c}$ )

94I 25c black on yellow (lower half of $50 \mathrm{Oc}$ )

e. 5oc stamps cut diagonally in two, each half being used as a $25 \mathrm{c}$ stamp.

$94225 \mathrm{c}$ black on yellow (upper right half of $50 \mathrm{Oc}$ )

$94325 \mathrm{c}$ black on yellow (lower right half of 50c)

$94425 \mathrm{c}$ black on yellow (upper left half of $50 \mathrm{Oc}$ )

$94525 \mathrm{c}$ black on yellow (lower left half of 5oc)

f. With period after I2.

946 I 2c black on yellow yellow

947 I2c black on green

We have never seen any used specimens without surcharge.

$\mathbf{2}^{\circ}$ Rounded saw tooth perforation.

$948 \quad 6 \mathrm{c}$ black on light brown

949 I2c black on yellow green

950 r2c black on sea green

$95 \mathrm{I} \quad 25 \mathrm{c}$ blue on light pink

$952 \quad 25 \mathrm{c}$ bluish black on light pink

953 50c black on yellow

954 IOOc black on red brown

Varieties :

a. Error of color.

955 I OOc brown on brown

b. rooc stamps divided in fourths, each

fourth being used as a $25 \mathrm{c}$ stamp.

$95625 \mathrm{cblack}$ on brown (upper right fourth of IOOC)

$95725 \mathrm{c}$ black on brown (lower right fourth of IOOC)

$95^{8} 25 \mathrm{c}$ black on brown (upper left fourth of IOOC)

$95925 \mathrm{c}$ black on brown (lower left fourth of 100c)

c. 5oc stamps cut vertically in two, each half being used as a $25 \mathrm{c}$ stamp.

$960 \quad 25 \mathrm{c} \mathrm{black}$ on yellow (right half of $50 \mathrm{c}$ )

96r $25 \mathrm{c}$ black on yellow (left half of $50 \mathrm{c}$ )

d. $50 \mathrm{c}$ stamps cut horizontally in two, each half being used as a $25 \mathrm{c}$ stamp.

$962 \quad 25 \mathrm{c}$ black on yellow (upper half of $50 \mathrm{c}$ ) $96325 \mathrm{c}$ black on yellow (lower half of $50 \mathrm{c}$ ) e. 5oc stamps cut diagonally in two, each half being used as a $25 \mathrm{c}$ stamp.

$96425 \mathrm{c}$ black on yellow (upper right half of 5Oc)

$96525 \mathrm{c}$ black on yellow (lower right half of $50 \mathrm{Oc}$ )

$96625 \mathrm{c}$ black on yellow (upper left half of $50 \mathrm{Oc}$ )

$96725 \mathrm{c}$ black on yellow (lower left half of $50 c$ )

f, Without period after I2.

968 I2c black on yellow green

969 I 2c black on sea green

$3^{\circ}$ Regularly perforated Io

$970 \quad 6 \mathrm{c}$ black on light brown ?

972 I2c black on sea green

$97325 \mathrm{c}$ blue on light pinls

$97425 \mathrm{c}$ bluish black on light pink ?

975 50c black on yellow ?

976 IOOc black on red brown?

We have only seen two specimens of the I2c with this perforation, both surcharged "24 Cuernavaca 68." 


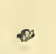


B. Plate II.

$A$. Ordinary wove paper.

I. Without surcharge. Unperforated.

$9776 \mathrm{c}$ black on light brown

978 I2c black on yellow green

979 I2c black on sea green

$980 \quad 25 \mathrm{c}$ blue on light pink

$98 \mathrm{x} .25 \mathrm{c}$ bluish black on light pink

$98250 \mathrm{c}$ black on yellow

983 Iooc black on red brown

We have never seen any specimens without surcharge perforated in any way, and, although they may exist in this condition, they can only be considered as unfinished stamps.

II. Surcharged with name of town only.

$$
\text { I } 0 \text { Unperforated. }
$$

$98425 \mathrm{c}$ blue on pink

$2^{\circ}$ Rounded saw tooth perforation.

985 I 2 c black on yellow green

The other values may exist in this condition, but they are very raxe. The only surcharge known is VERA CRUZ, I5 $52 \mathrm{~mm}$.

III. Surcharged with number and abbreviated date only on right hand side

$I^{\circ}$ Unperforated.

$986 \quad 6 \mathrm{c}$ black on light brown

987 I2c black on yellow green

988 I2c black on sea green

$98925 \mathrm{c}$ blue on light pink

$990 \quad 25 \mathrm{C}$ blue on very light pink

99I $50 \mathrm{c}$ black on yellow

992 tooc black on red brown

Varieties.

a. Error of color.

993 Iac black on light brown

994. $50 \mathrm{c}$ blue on light pink

b. No period after figure of value.

$99525 \mathrm{C}$ blue on light pink

$99625 \mathrm{c}$ blue on very light pink

$99750 \mathrm{c}$ black on yellow

998 rooc black on red brown

The tooc brown on brown does not exist in plate II. although catalogued so by $\mathrm{Me}$ keel.

$2^{\circ}$ Rounded saw tooth perforation. $6 \mathrm{c}$ black on light brown $12 \mathrm{c}$ black on yellow green I $2 \mathrm{c}$ black on sea green $25 \mathrm{c}$ blue on light pink $25 \mathrm{c}$ blue on very light pink

$100325 \mathrm{c}$ blue on very light

1005 rooc black on red brown Varizites:

a. Error of color,

1006 12c black on light brown

b. No period after figure of value.

$100725 \mathrm{c}$ blue on light pink

$100825 \mathrm{c}$ blue on very light pink

I009 $50 \mathrm{Oc}$ black on yellow

roIO IOOc black on red brown

$3^{\circ}$ Square perforation.

IOII $6 \mathrm{c}$ black on light brown

IOI2 I2c black on yellow green

IOI3 i2c black on sea gxeen

IOI4. $25 \mathrm{c}$ blue on light pink

IOI5 $25 \mathrm{C}$ blue on very light pink

IoI6 soc black on yellow

IOI7 rooc black on red brown
Varieties:

a. Error of color.

roI8 I2c black on light brown

b. No period after figure of value.

roI9 $25 \mathrm{c}$ blue on light pink

IO2O $25 \mathrm{c}$ blue on very light pink

ro2 I $50 \mathrm{c}$ black on yellow

I022 Iooc black on red brown

$4^{\circ}$ Pin perforated $\mathrm{I} 3$.

ro23 6c black on light brown

1024 $12 \mathrm{c}$ black on yellow green

IO25 I2c black on sea green

I026 $25 \mathrm{c}$ blue on light pink

I027 $25 \mathrm{c}$ blue on very light pink

I028 $50 \mathrm{c}$ black on yellow

I029 rooc black on red brown

Varisites: No period after figure of value.

I030 $25 \mathrm{c}$ blue on light pink

IO3 $\mathrm{r} 25 \mathrm{c}$ blue on very light pink

I032 50 c black on yellow

I033 rooc black on red brown

$5^{\circ}$ Pin perforated $x 4$.

I034 $6 \mathrm{c}$ black on light brown

I035 I2c black on yellow green

I036 I2c black on sea green

I037 $25 \mathrm{c}$ blue on light pink

1038 25c blue on very light pink

I039 50c black on yellow

I040 IOOc black on red brown

Varieties: No period after figure of value.

104I $25 \mathrm{C}$ blue on light pink

I042 $25 \mathrm{c}$ blue on very light pink

IO +35 Oc black on yellow

Io 14 IOOc black on red brown

$6^{\circ}$ Pin Perforated I5. $_{5}$

I045 6c black on light brown

I046 I2c black on yellow green

I047 I $2 \mathrm{c}$ black on sea green

I048 $25 \mathrm{c}$ blue on light pink

I049 $25 \mathrm{c}$ blue on very light pink.

I050 $50 \mathrm{O}$ black on yellow

I05 I rooc black on red brown

Varieties: No period after figure of value. I052 $25 \mathrm{C}$ blue on light pink

I053 $25 \mathrm{c}$ blue on very light pink

x054 $50 \mathrm{c}$ black on yellow

I055 Iooc black on red brown

IV. Surcharged with number and abbreviated date on right hand side of stamp with the addition of the name of the postal district.

$I^{\circ}$ Unperforated.

I056 $6 \mathrm{c}$ black on light brown

I057 I2c black on yellow green

I058 I $2 \mathrm{c}$ black on sea green

1059 $25 \mathrm{c}$ blue on light pink

I060 $25 \mathrm{c}$ blue on very light pink

I06I $50 \mathrm{c}$ black on yellow

I062 rooc black on red brown

Varieties:

a. Error of color.

I063 I2c black on light brown

b. No period after figure of value.

$106425 \mathrm{c}$ blue on light pink

I065 $25 \mathrm{c}$ blue on very light pink

I066 $50 \mathrm{c}$ black on yellow

1067 rooc black on red brown

$2^{\circ}$ Rounded saw tooth perforation

I068 $6 \mathrm{c}$ black on light brown

I069 I2c black on yellow green

I070 I2c black on sea green

IO7 I $25 \mathrm{c}$ blue on light pink

I $07225 \mathrm{c}$ blue on very light pink

ro73 50c black on yellow

I074 Iooc black on red brown 
Varieties:

a. Error of color.

I075 I2c black on light brown

b. No period after figure of value.

I076 $25 \mathrm{c}$ blue on ligh pink

I077 $25 \mathrm{c}$ blue on very light pink

I078 $50 \mathrm{c}$ black on yellow

I079 rooc black on red brown

$3^{\circ}$ Square perforation.

I080 $6 \mathrm{c}$ black on light brown

I08I I $2 \mathrm{c}$ black on yellow green

I082 I2c black on sea green

$108325 \mathrm{c}$ blue on light pink

I084 $25 \mathrm{C}$ blue on very light pink

I085 50c black on yellow

I086 rooc black on red brown

Varieties:

a. Error of color.

I087 I 2c black on light brown

b. No period after figure of value.

I088 $25 \mathrm{C}$ blue on light pink

I089 $25 \mathrm{c}$ blue on very light pink

IOgO $50 \mathrm{c}$ black on yellow

IOgI IOOc black on red brown

$4^{\circ}$ Pin Perforated I 3.

I092 $6 \mathrm{c}$ black on light brown

I093 I $2 \mathrm{c}$ black on yellow green

Io94 I2c black on sea green

I095 $25 \mathrm{c}$ blue on light pink

I096 $25 \mathrm{c}$ blue on very light pink.

I097 50c black on yellow

I098 rooc black on red brown

Varieties: No period after figure of value.

rog9 $25 \mathrm{c}$ blue on light pink

I IOO $25 \mathrm{c}$ blue on very light pink

r ror $50 \mathrm{Oc}$ black on yellow

I IO2 IOOc black on red brown

$5^{\circ}$ Pin perforated 14 .

I $1036 \mathrm{c}$ black on light brown

I IO4 I $2 \mathrm{c}$ black on yellow green

I IO5 I2c black on sea green

I $10625 \mathrm{c}$ blue on light pink

I IO7 $25 \mathrm{c}$ blue on very light pink

I 108 50c black on yellow

IIO9 IOOc black on red brown

Varieties: No period after figure of value.

I I Io $25 \mathrm{c}$ blue on light pink

I III $25 \mathrm{c}$ blue on very light pink

II 25 oc black on yellow

III3 IOOc black on red brown

$6^{\circ}$ Pin perforated 15 .

III4 $6 \mathrm{c}$ black on light brown

I I 15 I2c black on yellow green

I I 6 I2c black on sea green

III $725 \mathrm{c}$ blue on light pink

I I $825 \mathrm{c}$ blue on very light pink

III9 $50 \mathrm{c}$ black on yellow

I 20 Iooc black on red brown

Varielies: No period after figure of value.

I I 2 I $25 \mathrm{c}$ blue on light pink

I $12225 \mathrm{C}$ blue on very light pink

I 23 5oc black on yellow

II 24 IOOc black on red brown

$7^{\circ}$ Pointed saw tooth perforation.

I125 25c blue on light pink (I Mexico 70)

$8^{\circ}$ Regular perforation 19.

I $2625 \mathrm{c}$ blue on light pink (I J Jalapa 68)

$B$. Very thin pelure paper.

I. Surcharged with number and abbreviated date.
$\mathrm{I}^{\circ}$ Unperforated.

I $2725 \mathrm{c}$ blue on light pink

$2^{\circ}$ Pin perforated.

II $2825 \mathrm{c}$ blue on light pink

II. Surcharged with number and abbreviated date in addition to the name of the postal district.

I U Unperforated.

I $29.25 \mathrm{c}$ blue on light pink.

$2^{\circ}$ Pin perforated.

I $13025 \mathrm{c}$ blue on light pink

$\mathrm{Mr}$. DeCoppet says that all the values are to be found on this paper. This, undoubtedly, is a mistake, as we have never seen any other ones in the immense stock which has gone through our hands.

Very thick paper-almost cardboard.

I. Surcharged with number and abbreviated date only. I U Unperforated.

II3I I2c black on yellow green $2^{\circ}$ Rounded saw tooth perforation.

1132 12c black on yellow green $3^{\circ}$ Pin Perforated 14.

II33 I2c black on yellow green $4^{\circ}$ Square perforation.

I I34 I $2 \mathrm{c}$ black on yellow green

II. Surcharged with number and abbreviated date with the addition of the name of the postal district.

$\mathrm{J}^{\circ}$ Unperforated.

I 135 r.2 black on yellow green

$2^{\circ}$ Rounded saw tooth perforation.

II36 I $2 \mathrm{c}$ black on yellow green $3^{\circ}$ Pin perforated.

I 37 r $2 \mathrm{c}$ black on yellow green $4^{\circ}$ Square perforation.

II 38 rac black on yellow green

We do not believe that any other values exist on this paper.

Some compilers catalogue the following errors:

I $2 \mathrm{c}$ black on yellow

Igc black on green

5 oc black on green

IIOc black on reddish brown

$85 \mathrm{c}$ blue on rose

The first and third ones are undoubtedly color changelings, while the second and fourth are merely defective impressions. The last one we believe to be an essay.

Names of Postal districts found on this issue.

Numbered from I to $4 \mathrm{I}$.

I 321 PXICO $15 \times 2 ; M 21 / 2$.

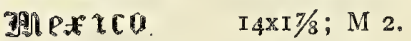

2 VERACRUZ $141 / 2 \times 11 / 2$.

VERACRIJ\% I $3 \frac{1}{2} \times 2$.

VERACRUZ I5 $\mathrm{x} 2$.

GUADALAJARA. 20\%2, with period.

3 GUADALA JARA. 22XI3/4, with period.

CUADALAJABA $20 \times 13 / 4$.

GUADALAJARA I $8 \times 13 / 4$. 




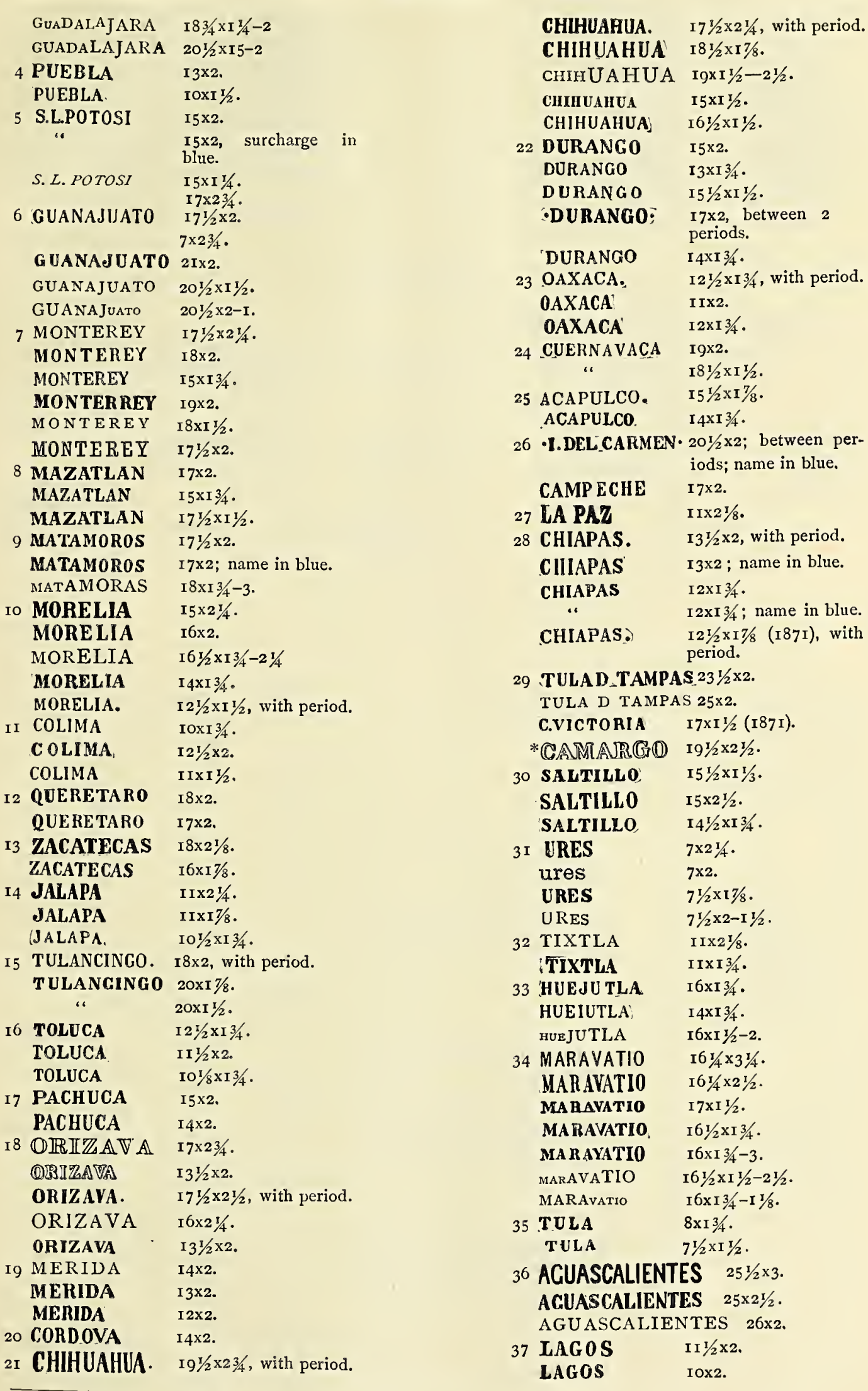

*This surcharge on the 6c, Type I., unperforated is in the collection of Mr. Albert E. Lawrence. 


\begin{tabular}{|c|c|c|c|c|}
\hline & LAGOS & I I $1 / 2 \times 13 / 4$. & 40 TLAXCALA & $161 / 2 \times 2$ \\
\hline & LAGOS & $8 \mathrm{x} / 2 \times 13 / 4$. & GUADALA JARA. & $20 \times 2$, with period. \\
\hline & LAGOS & I I $1 / 2 \times 13 / 4-23 / 4$. & 4 I GUADALAJARA. & $20 \times 13 / 4$ \\
\hline 38 & TABASCO & $141 / 2 \times 13 / 4$ & GUADALAJARA. & $20 \times 1 / 2$, with period. \\
\hline & TABASCO & $121 / 2 \times 13 / 4$. & CUADALAJARA & $2 \mathrm{I} \times 2 \mathrm{I} / 2$. \\
\hline 39 & TAMPICO & $14 \frac{1}{2} \times 2$ & CUADALAJARA. & I $81 / 2 \times 11 / 2$, with period. \\
\hline & TAM PICO & I $41 / 2 \times I_{3} / 4$, with period. & GUADALAJARA & $201 / 2 \times 15 / 8-21 / 2$ \\
\hline & $\begin{array}{l}\text { TAMPICO } \\
\text { TAMPICO }\end{array}$ & $\begin{array}{l}\text { I } 3 \times 13 / 4-3 \\
\text { I } 21 / 2 \times I 3 / 4(\text { I } 87 I)\end{array}$ & GUADALAJARA & $22 \times 13 / 4$ \\
\hline
\end{tabular}

\section{HABILITADOS.}

Surcharged with names of $\mathbf{2}$ postal districts.

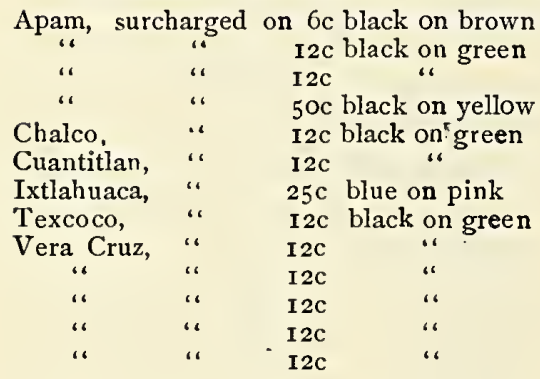
On type I, Mexico,

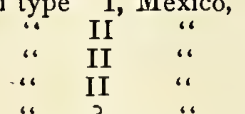

I-6o rounded saw tooth perf

I-70 pin perforated.

I -7 I square perf.

I-69 rounded saw tooth perf.

I-? ?

“ II $\quad$ II Toluca, $\quad$ I6-70 unperforated.

“ “. II $\quad$ II Toluca, $\quad$ I6-72 pin perforated.

“ II Mexico, I- 7 I pin perforated.

"? I-? ? IHeitman's

"? " I-? ? ? Handbuch

“ II Cuernavaca, 24-7I unperforated.

" ? Morelia, Io-? ? ?

Moens.

ERRORS.

Errors in numbering.

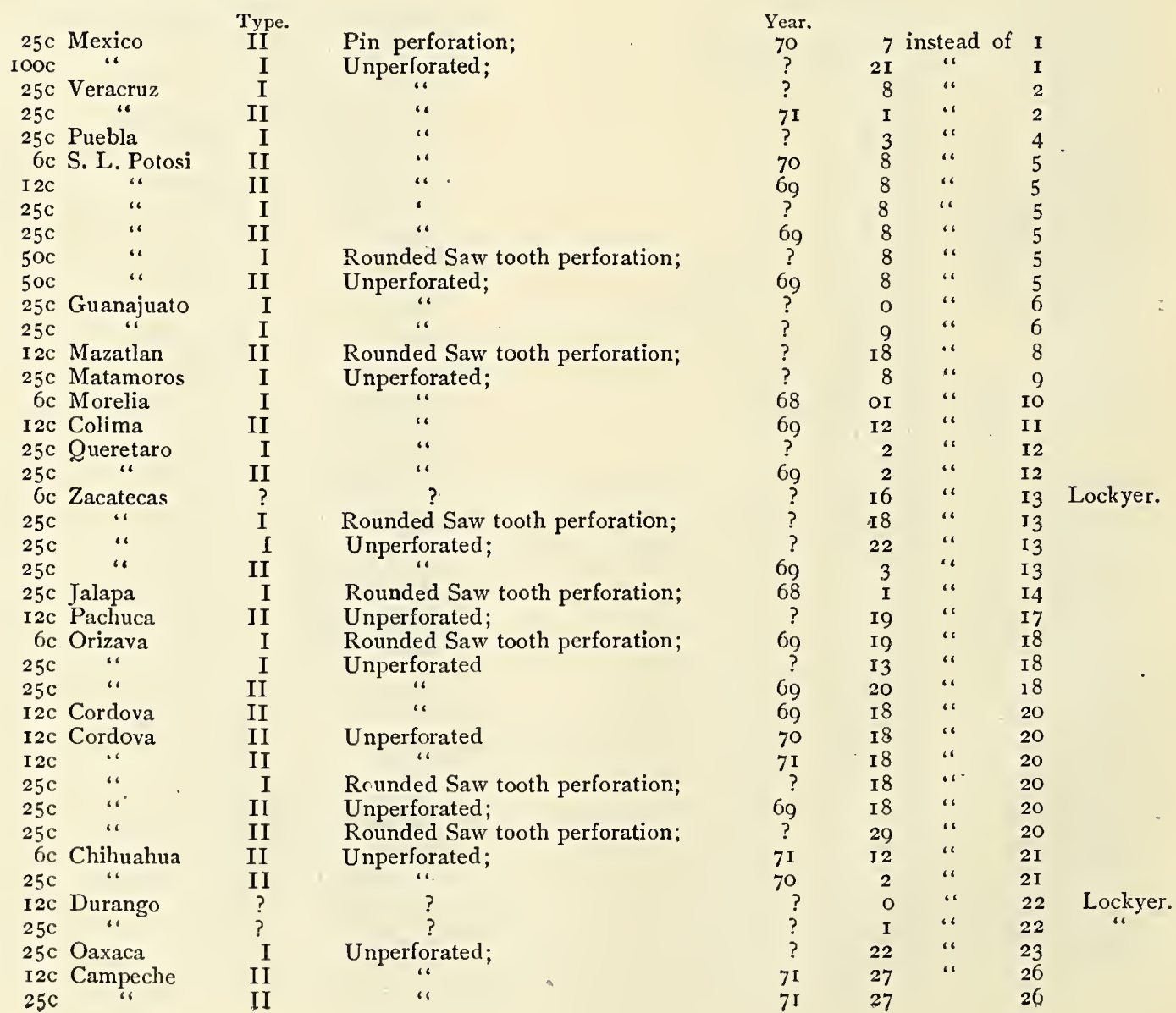


. 、 



\begin{tabular}{|c|c|c|c|c|c|c|c|c|}
\hline \multicolumn{4}{|c|}{ Type. } & \multicolumn{5}{|c|}{ Year. } \\
\hline $25 \mathrm{c}$ & Tula de Tampa & s I & Unperforated & ? & 28 & steas & 2 & \\
\hline $25 \mathrm{c}$ & & I & Pin perforation; & 68 & 28 & " & 2 & \\
\hline $6 c$ & Tixtla & II & Unperforated; & $?$ & 23 &.. & 3 & \\
\hline $25 \mathrm{c}$ & Huejutla & I & - & 70 & 23 & “، & & \\
\hline $25 \mathrm{c}$ & 4 & II & “. & $?$ & 38 & “" & 3 & \\
\hline $25 \mathrm{c}$ & "“ & II & ،. & 70 & 22 & “" & & \\
\hline IOOC & “ & II & “" & $?$ & 36 & ." & 3 & \\
\hline I $2 \mathrm{C}$ & Maravatio & I & “ & $?$ & $3 I$ & “ & 3 & \\
\hline I $2 \mathrm{c}$ & “" & ? & ? & ? & 32 & “" & & Lockyer. \\
\hline $6 c$ & Aguascalientes & II & Unperforated; & 70 & 39 & “" & & \\
\hline $25 \mathrm{c}$ & $"$ " & II & “" & 69 & I 2 & “، & & \\
\hline $25 \mathrm{c}$ & Lagos & II & “" & 71 & 9 & “" & & \\
\hline $\mathrm{I} 2 \mathrm{C}$ & Tabasco & I & “ & 68 & 39 & "“ & & \\
\hline I2c & Tampico & I & “" & ? & 33 & "“ & & \\
\hline I $2 \mathrm{c}$ & Guadalajara & ? ? & ? & ? & I 4 & " & & Lockyer. \\
\hline IOOC & 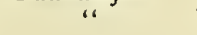 & ? ? & ? & ? & I4 & “" & & \\
\hline
\end{tabular}

Errors in the position of Surcharge of No. and date.

Surcharged on left hand side of stamp, thus:

\begin{tabular}{|c|c|c|c|c|c|}
\hline District. & Value. & Nos. Date & Type. & Perforation. & $\begin{array}{l}\text { Measurement of } \\
\text { Surcharge. }\end{array}$ \\
\hline Mexico & $25 \mathrm{c}$ & $1-70$ & II & Pin perforated & $14 \times 17 / 8$ \\
\hline "1 & $25 \mathrm{C}$ & $1-7 I$ & II & "1i perata & $15 \times 2$ \\
\hline “ & $25 \mathrm{c}$ & $I-72$ & II & “ & $15 \times 2$ \\
\hline “ & $5 \mathrm{Oc}$ & I- -70 & ? & ? & $?$ \\
\hline Vera Cruz & $6 c$ & $2-70$ & II & Unperforated & I $5 \times 2$ \\
\hline “ & $\mathrm{I} 2 \mathrm{C}$ & $2-70$ & I & "“ & $?$ \\
\hline “" & I $2 \mathrm{c}$ & $2-68$ & I & Rounded Saw tooth & I $5 \times 2$ \\
\hline “. & $12 \mathrm{C}$ & $2-70$ & II & Unperforated & I $5 \times 2$ \\
\hline$"$ " & IOOC & $2-70$ & I & “ & $?$ \\
\hline Guanajuato & $25 \mathrm{c}$ & $6-70$ & II & “" & I $81 / 2 \times 2$ \\
\hline Mazatlan & $25 \mathrm{c}$ & $8-70$ & II & “" & $15 \times 13 / 4$ \\
\hline Morelia & $25 \mathrm{c}$ & $10-70$ & I & “" & $?$ \\
\hline "“ & $25 \mathrm{c}$ & $10-70$ & II & “" & $I 4 X_{1} 3 / 4$ \\
\hline “" & $50 \mathrm{c}$ & $10-70$ & $\mathrm{I}$ & Rounded Saw tooth & $?$ \\
\hline Queretaro & $25 \mathrm{c}$ & $12-70$ & II & Unperforated & I $7 \times 2$ \\
\hline Zacatecas & $25 \mathrm{c}$ & $13-70$ & $\mathrm{I}$ & I & $?$ \\
\hline "، & $25 \mathrm{c}$ & $13-70$ & II & “، & $16 \times 17 / 8$ \\
\hline Tulancingo & $25 \mathrm{c}$ & I $5-70$ & II & “" & I $8 \times 2$ with period \\
\hline Cordova & $25 \mathrm{c}$ & $20-70$ & II & “ & $\mathrm{I} 2 \mathrm{XI} 3 / 4$ \\
\hline Durango & $25 \mathrm{c}$ & $22-70$ & II & “" & $13 \times 13 \%$ \\
\hline Oaxaca & $25 \mathrm{c}$ & $23-70$ & II & “ & IIX2. \\
\hline Acapulco & $25 \mathrm{c}$ & $25-70$ & II & ، & I 4 XI $7 / 8$ \\
\hline Saltillo & $6 c$ & $30-69$ & I & “، & $?$ \\
\hline "1 & $\mathrm{I} 2 \mathrm{c}$ & $30-68$ & II & ، & $141 / 2 \times 13 / 4$ \\
\hline Huejutla & $25 \mathrm{C}$ & $* 22-70$ & II & “ & I $4 \times 13 / 4$ \\
\hline “ & $25 \mathrm{c}$ & $33-70$ & II & “" & $\mathrm{I} 4 \mathrm{XI} 3 / 4$ \\
\hline Tampico & $25 \mathrm{c}$ & $39-70$ & I & “" & 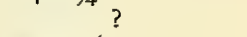 \\
\hline Guadalajara & $25 \mathrm{c}$ & $41-70$ & II & “. & $2 \mathrm{IXI} 3 / 4$ \\
\hline
\end{tabular}

*Error of No. 22 instead of 33.

Surcharged on right hand side of stamp, thus :

\begin{tabular}{|c|c|c|c|c|c|}
\hline District. & Value. & Nos. Date & Type. & Perforation. & $\begin{array}{l}\text { Measurement of } \\
\text { Surcharge. }\end{array}$ \\
\hline $\begin{array}{l}\text { Vera Cruz } \\
\text { S. L. Potosi } \\
\text { Guanajuato }\end{array}$ & $\begin{array}{r}6 c \\
25 c \\
25 c \\
25 c\end{array}$ & $\begin{array}{l}2-68 \\
5-68 \\
6-68 \\
6-68\end{array}$ & $\begin{array}{l}\text { I } \\
\text { I } \\
\text { I } \\
\text { I }\end{array}$ & Rounded Saw tooth & $\begin{array}{l}15 \times 2 \\
15 \times 2 \text { blue } \\
171 / 2 \times 23 / 4 \\
2 \text { I X2 }\end{array}$ \\
\hline
\end{tabular}

Surcharged on left hand side of stamp, thus: 


\begin{tabular}{|c|c|c|c|c|c|}
\hline District. & Value. & Nos. Date & Type. & Perforation. & $\begin{array}{l}\text { Measurcment of } \\
\text { Surcharge. }\end{array}$ \\
\hline $\begin{array}{l}\text { Mexico } \\
\text { S. L. Potosi } \\
\text { Queretaro } \\
\text { Huejutla }\end{array}$ & $\begin{array}{l}50 c \\
25 \mathrm{c} \\
12 \mathrm{c} \\
12 \mathrm{c}\end{array}$ & $\begin{array}{r}1-69 \\
5-68 \\
12-68 \\
33-68\end{array}$ & $\begin{array}{l}\text { I } \\
\text { I } \\
\text { I } \\
\text { I }\end{array}$ & $\begin{array}{l}\text { Rounded Saw tooth } \\
\text { "“" } \\
\text { Square perforation } \\
\text { Pin perforated }\end{array}$ & $\begin{array}{l}\text { I5X2 black } \\
\text { No Surcharge }\end{array}$ \\
\hline
\end{tabular}

Various.

as per illustration.

I2C

$25 \mathrm{c}$

$25 \mathrm{C}$

$25 \mathrm{C}$

$25 \mathrm{C}$

II. Wrong placing of No. and date o 397 Nos and dates,
I.<smiles>[3H]</smiles>

II.

I.

3 Nos. and dates, thus:

double impression of Nos, and dates.

double impression of Nos. and dates.

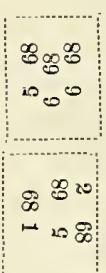

$0^{\infty}$
$0^{\infty}$
90
208

Unperf. No dist. name.

Unperf, No dist. name.

Rounded saw Guanajuato, tooth perf.

Unperf.

Tampico.

Unperf. Chihuahua.

Mexico.

I $81 / 2 \times$ I $7 / 8$

Pin Perf. Mexico.

Unperf. Morelia.

Tulancingo.

“ II

"II.

Wiih number printed thus:

i० 72

With letter I missing in name SAL ' 7 I

?"

Saltillo.

(Heilman's Handbuch and Lockyer note this)

“ II. Double surcharge of name

$\begin{array}{ll}\text { Unperf. } & 4 \text { Puebla } 69 \\ \text { Square } & \mathrm{N} \text { instead of }\end{array}$

I $2 \times 17 \% / 2$ 



\begin{tabular}{|c|c|c|c|}
\hline \multirow{2}{*}{\multicolumn{2}{|c|}{ Value. }} & \multicolumn{2}{|c|}{ District. } \\
\hline & & \multicolumn{2}{|c|}{ Mexico } \\
\hline 250 & & \multicolumn{2}{|c|}{ Mexico } \\
\hline I $2 \mathrm{C}$ & & \multicolumn{2}{|c|}{ Vera Cruz } \\
\hline 120 & & \multicolumn{2}{|c|}{ Vera Cruz } \\
\hline 250 & & \multicolumn{2}{|c|}{ Vera Cruz } \\
\hline $12 C$ & & \multicolumn{2}{|c|}{ Guadalajara } \\
\hline I2C & & \multicolumn{2}{|c|}{ S. I. Potosi } \\
\hline $\begin{array}{r}\text { I } 20 \\
6 c\end{array}$ & & \multicolumn{2}{|c|}{$\begin{array}{l}\text { Guanajuato } \\
\text { Oaxaca }\end{array}$} \\
\hline $\begin{array}{r}120 \\
60 \\
60\end{array}$ & & \multicolumn{2}{|c|}{$\begin{array}{l}\text { Campeche } \\
\text { La Paz }\end{array}$} \\
\hline 250 & & \multicolumn{2}{|c|}{$\begin{array}{l}\text { Ures } \\
\text { Tampico }\end{array}$} \\
\hline & & Type. & No. \\
\hline 25 & cent & III. & I \\
\hline 12 & $"$ & II. & 2 \\
\hline 12 & " & II. & 2 \\
\hline 25 & “. & I. & 4 \\
\hline 12 & “ & II. & 4 \\
\hline I2 & "“ & II. & 5 \\
\hline 12 & “ & $?$ & 5 \\
\hline 25 & 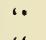 & I. & 5 \\
\hline 25 & “ & $?$ & 5 \\
\hline 12 & “ & II. & 6 \\
\hline 25 & “ & II. & 6 \\
\hline 25 & “ & I. & 7 \\
\hline 25 & “ & I. & 8 \\
\hline 12 & 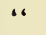 & I. & I6 \\
\hline 12 & “ & II. & I6 \\
\hline I 2 & “. & $?$ & 34 \\
\hline 25 & “ & $?$ & 34 \\
\hline 12 & “ & I. & 39 \\
\hline 25 & ‘ & I. & 39 \\
\hline
\end{tabular}

Names of Postal Districts Surcharged Twice.

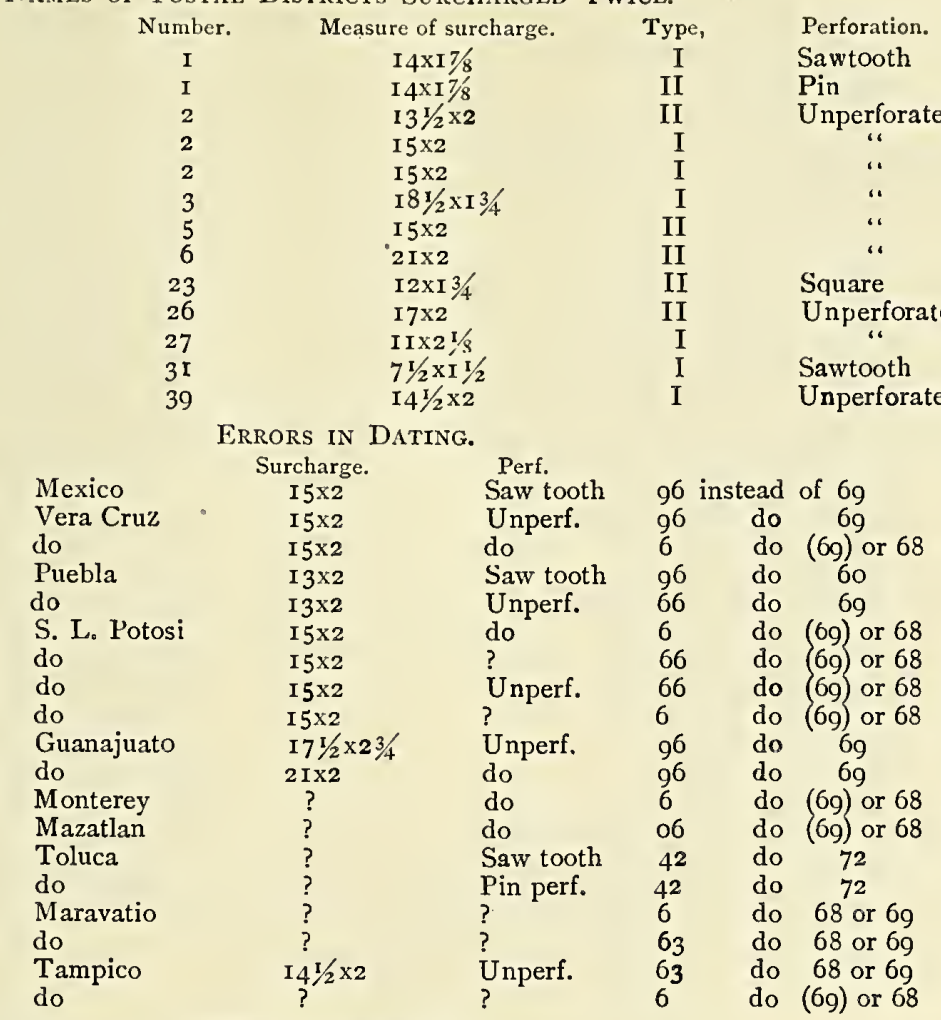

Double perforation vertically.

Double Perforation.

Double perforation horizontally.

$$
\begin{aligned}
& \text { I2 cent, type I. No. I } \\
& \text { “II. “" } 31 \text { Vres Cruz } \\
& \text { “ “ II. “ } 32 \text { Tixtla } \\
& \text { “. “ I. “ “ } 33 \\
& \text { “ II. “" } 8 \text { Mazatlan } \\
& \text { “II. “ } 5 \text { S. L. Potosi, } 70 \\
& \text { "I. " I } 2
\end{aligned}
$$

I 872

Same type as preceding issue but surcharged Anotado* as per cut; all are of plate II.

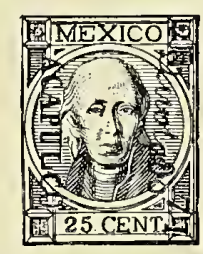

I. Surcharged with number and date only. $\mathrm{I}^{\circ}$ Unperforated.

I $396 \mathrm{c}$ black on light brown

I4O $12 \mathrm{c}$ black on yellow green

II 4 I $2 \mathrm{c}$ black on sea green

I $4225 \mathrm{c}$ blue on light pink

I $4325 \mathrm{c}$ blue on very light pink

I 445 oc black on yellow

I I 5 IOOc black on red brown

Varieties: No period after figure of value.

I $46 \quad 25 \mathrm{c}$ blue on light pink

I I $4725 \mathrm{C}$ blue on very light pink

II 48 5Oc black on yellow

II 49 rooc black on red brown

$2^{\circ}$ Rounded sawtooth perforation.

II50 $6 \mathrm{c}$ black on light brown

I 5 I I2c black on yellow green

I 52 I2c black on sea green
68 square perf. 12 cent, type II. No. 2 Vera Cruz 7 I square perf.

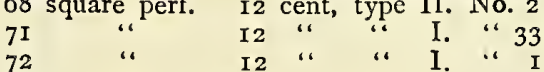

“

“،

“

II $5325 \mathrm{c}$ blue on light pink

II $5425 \mathrm{C}$ blue on very light pink

I 55 50c black on yellow

II56 rooc black on red brown

Varieties: No period after figure of value

II $5725 \mathrm{c}$ blue on light pink

I $58 \quad 25 \mathrm{c}$ blue on very light pink

I I59 $50 \mathrm{c}$ black on yellow

I 160 IOOc black on red brown

$3^{\circ}$ Pin perforated 14.

II6I 6c black on light brown

II62 I2c black on yellow green

I 63 I 2c black on sea green

II $6425 \mathrm{c}$ blue on light pink

I $6525 \mathrm{c}$ blue on very light pink

I 66 5oc black on yellow

II67 Iooc black on red brown

Varieties: No period after figure of value.

I $6825 \mathrm{c}$ blue on light pink

II $6925 \mathrm{c}$ blue on very light pink

I 70 5Oc black on yellow

I 7 I IOOc black on red $4^{\circ}$ Square perforation.

II $726 \mathrm{c}$ black on light brown

II 73 I2c black on yellow green

I 74 I 2c black on sea green

I $7525 \mathrm{c}$ blue on light pink

II $7625 \mathrm{c}$ blue on very light pink

I 775 oc black on yellow

I 78 IOOc black on red brown 
Varieties: No period after figure of value.

I $7925 \mathrm{C}$ blue on light pink

I $80 \quad 25 \mathrm{c}$ blue on very light pink

I 8 I $50 \mathrm{c}$ black on yellow

II82 Iooc black on red brown

II. Surcharged with number and date and name of postal district.

$$
\mathbf{I}^{\circ} \text { Unperforated. }
$$

II 83 6c black on light brown

II84 I2c black on yellow green

II85 I2c black on sea green

I $8625 \mathrm{c}$ blue on light pink

II $8725 \mathrm{c}$ blue on very light pink

I 88 5oc black on yellow

II 89 rooc black on red brown

Varieties: No period after figure of value.

II $90 \quad 25 \mathrm{c}$ blue on light pink

I I9I $25 \mathrm{c}$ blue on very light pink

I I92 $50 \mathrm{C}$ black on yellow

I I93 IOOc black on red brown

$2^{\circ}$ Rounded saw tooth perforation.

Ir94 6c black on light brown

II95 I2c black on yellow green

I I96 I2c black on sea green

I $19725 \mathrm{c}$ blue on light pink

I I98 $25 \mathrm{c}$ blue on very light pink

II99 $50 \mathrm{c}$ black on yellow

I200 tooc black on red brown

Varieties: No period after figure of value.
I20I $25 \mathrm{c}$ blue on light pink

I $20225 \mathrm{C}$ blue on very light pink

I 203 5Oc black on yellow

I204 IOOc black on red brown

$3^{\circ}$ Pin perforated $\mathrm{r} 4$.

I205 $6 \mathrm{c}$ black on light brown

I206 I2c black on yellow green

I207 I 2C black on sea green

I $20825 \mathrm{C}$ blue on light pink

I209 25C blue on very light pink

I2 IO $50 \mathrm{c}$ black on yellow

I2I I IOOc black on red brown

Varieties: No period after figures of value.

I 2 I $225 \mathrm{c}$ blue on light pink

I213 $25 \mathrm{c}$ blue on very light pink

I2 I4 5Oc black on yellow

I2I 5 IOOc black on red brown

$4^{\circ}$ Square perforation.

121 6 6 black on light brown

I217 I2c black on yellow green

I2I 8 I2c black on sea green

12 I9 $25 \mathrm{c}$ blue on light pink

I220 $25 \mathrm{C}$ blue on very light pink

I22 I 5 Oc black on yellow

I222 IOOc black on red brown

Varieties: No period after figure of value.

I223 $25 \mathrm{C}$ blue on light pink

$122425 \mathrm{c}$ blue on very light pink

I225 50c black on yellow

I226 IOOc black on red brown

callo stock of the stamps of the new issue was found too small to supply all the postofices the Government caused some of the stamps of 1868 (which had already been withdrawn from circulation) to be surcharged $A N O T A D O$, which means: noted, registered; and replaced into circulation.

List of stamps we have seen with the surcharge "Anotado."

\begin{tabular}{|c|c|c|c|c|c|c|}
\hline \multirow{4}{*}{ I } & \multirow{2}{*}{ Mexico, } & \multirow{2}{*}{$\begin{array}{l}\text { Type, } \\
\text { I } 5 \times 2\end{array}$} & \multicolumn{2}{|c|}{ Value. } & Saw tooth Perforation. & \multirow{2}{*}{$\begin{array}{c}\text { Year. } \\
72 \\
72\end{array}$} \\
\hline & & & I 2 & "6 & Pin & \\
\hline & & “ & 25 & " & Pin & $7 I$ \\
\hline & “" & “ & 25 & “ & Pin & 72 \\
\hline \multirow[t]{3}{*}{2} & Vera Cruz, & I5 52 & I2 & “" & Unperforated. & $7 \mathrm{I}$ \\
\hline & & $138 / 2 \times 2$ & 25 & “ & Square Perforation. & $7 \mathrm{I}$ \\
\hline & “ & $141 / 2 \times 11 / 2$ & 25 & “ & & 7 I \\
\hline \multirow[t]{4}{*}{4} & Puebla, & I I $1 / 2 \times I^{1} 1 / 2$ & I2 & “" & Unperforated. & 70 \\
\hline & “ & $13 \times 2$ & 12 & “ & “ & $7 \mathrm{I}$ \\
\hline & “ & $13 \times 2$ & 25 & “ & “" & $7 \mathrm{I}$ \\
\hline & “ & $\operatorname{IOXI} 1 / 2$ & 25 & “ & “" & $7 \mathrm{I}$ \\
\hline \multirow[t]{3}{*}{5} & S. L. Potosi, & $15 \times 2$ & 25 & $" “$ & “. & $7 \mathrm{I}$ \\
\hline & & “ & 25 & “ & Square Perforation. & $7 \mathrm{I}$ \\
\hline & Guanajuato, & 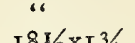 & 25 & “" & " " & $7 \mathrm{I}$ \\
\hline 6 & Guanajuato, & $\begin{array}{l}18 \mathrm{I} / 2 \times 13 / 4 \\
2 \mathrm{IX} 2\end{array}$ & $\begin{array}{l}25 \\
25\end{array}$ & $\because$ & Unperforated. & $\begin{array}{l}71 \\
71\end{array}$ \\
\hline \multirow[t]{2}{*}{7} & Monterey, & $15 \times 13 / 4$ & 25 & “ & ، & $7 x$ \\
\hline & & $18 \times 2$ & $2 \xi$ & “ & “" & $7 \mathrm{I}$ \\
\hline \multirow[t]{2}{*}{8} & Mazatlan, & $17 \times 11 / 2$ & 12 & “" & “" & 70 \\
\hline & “ & $15 \times 13 / 4$ & 25 & “ & “ & $7 \mathrm{I}$ \\
\hline 9 & Matamoros, & $\begin{array}{l}171 / 2 \times 2 \\
\text { in blue. }\end{array}$ & 25 & “ & “ & 70 \\
\hline \multirow[t]{3}{*}{ IO } & Morelia, & $14 \times 13 / 4$ & I2 & “ & “ & $7 I$ \\
\hline & $" ،$ & $"$ & 25 & “ & " & 71 \\
\hline & “" & $\because$ & 25 & “" & Square Perforation. & $7 \mathrm{I}$ \\
\hline I I & Colima, & $12 \frac{1}{2} \times 11 / 2$ & 25 & $"$ & Unperforated. & 71 \\
\hline \multirow{2}{*}{12} & $\begin{array}{l}\text { Colima, } \\
\text { Queretaro }\end{array}$ & $10 \times 13 / 4$ & $\begin{array}{l}25 \\
25\end{array}$ & “. & Souare Perforation. & 70 \\
\hline & $=0$ & $\begin{array}{c}17 \times 2 \\
-4\end{array}$ & 50 & ". & $\begin{array}{l}\text { Square fortoration. } \\
\text { Unperforated. }\end{array}$ & $\begin{array}{l}70 \\
71\end{array}$ \\
\hline \multirow[t]{2}{*}{13} & Zacatecas & $16 \times 17 / 8$ & 25 & “" & Unperforated. & 70 \\
\hline & “ & " & 25 & “ & Square Perforation. & $7 \mathrm{I}$ \\
\hline \multirow[t]{2}{*}{15} & Tulancingo & $18 \times 2$ & 25 & “. & Unperforated. & $7 x$ \\
\hline & & $20 \times 17 / 8$ & 25 & “ & “ & 71 \\
\hline 16 & Toluca & II $1 / 2 \times 13 / 4$ & 25 & “ & " & $7 \mathbf{I}$ \\
\hline \multirow[t]{2}{*}{ I7 } & Pachuca & $14 \times 2$ & 25 & 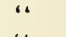 & " & $7 \mathrm{I}$ \\
\hline & “" & $“$ & 25 & “" & Square Perforation. & 70 \\
\hline $2 \mathrm{I}$ & Chihuahua & $161 / 2 \times 11 / 2$ & 25 & “ & " & $7 I$ \\
\hline \multirow[t]{5}{*}{22} & Durango & $13 \times 13 / 4$ & I. 2 & " & Unperforated. & $7 I$ \\
\hline & “ 2 periods. & $12 \times 2$ & 25 & “ & (1) “ & $7 \mathrm{I}$ \\
\hline & “ & I $4 \times 13 / 4$ & 25 & “. & “ & $7 \mathrm{I}$ \\
\hline & “" & “ & 25 & " & Square Perforation. & $7 I$ \\
\hline & & I $5 \times 2$ & 25 & “" & Unperforated. & 71 \\
\hline
\end{tabular}




\section{.}





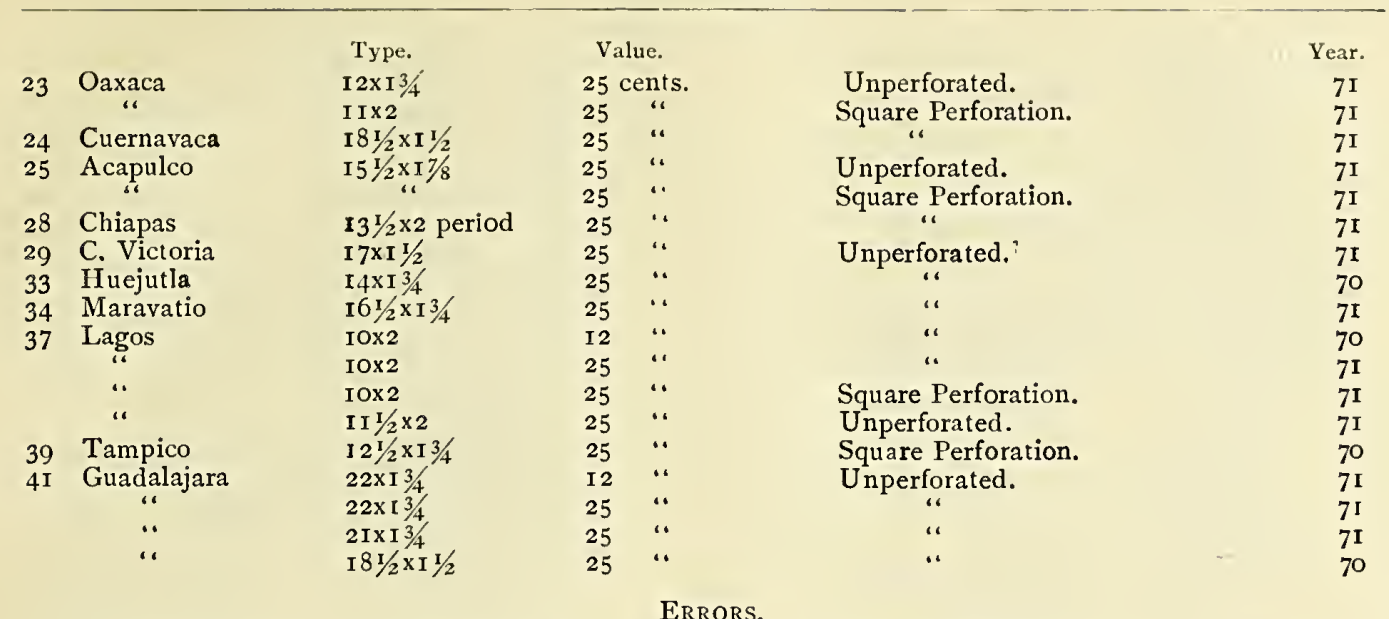

Double surcharge "Anotado" 25 c S. L. POTOSI, I $5 \times 2$ square perforation 7 I.

Vertical pair, the upper one without surcharge "Anotado," 25c I I COLIMA IOxI 3/4, 70 unperforated.

I872.

Head of Hidalgo to the left, lithographed on white paper of different kinds, with moiré pattern in blue on back of stamp. Size $191 / 2 \times 24 \mathrm{~mm}$.

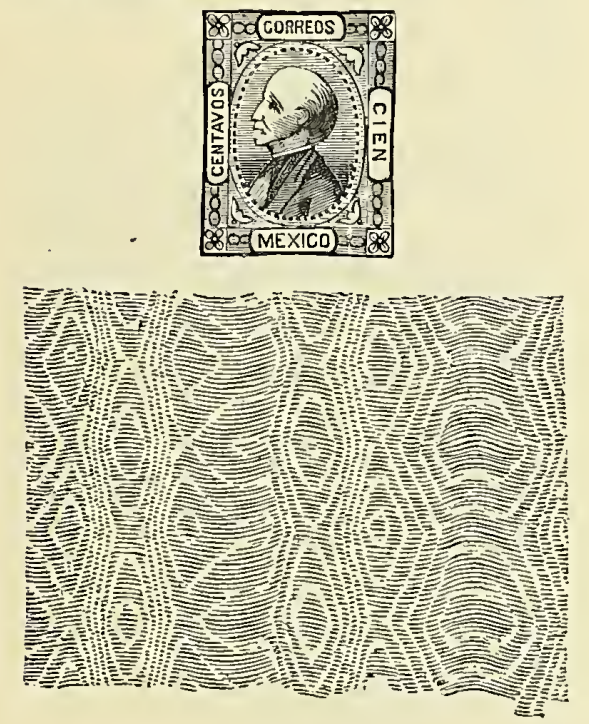

A. On white paper, laid vertically, surcharged with number and abbreviated date

and name of postal district.

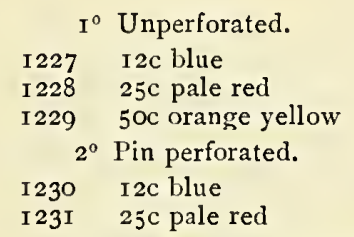

So far the following surcharges have been found on these stamps:

\begin{tabular}{|c|c|c|}
\hline I & fHll $x \dot{x} E$ o & I $5 \times 2 ;$ M $2 \mathrm{~T} / 2$ \\
\hline 2 & ACA PIILCO: & I $51 / 2 \times 17 / 8$; with period. \\
\hline I6 & CUADALAJARA & $\begin{array}{l}22 \times 23 / 4 ; A \text { and } J \text { in } \\
\text { italics. }\end{array}$ \\
\hline I6 & GUADALAJARA & $\begin{array}{l}201 / 2 \times 21 / 4 ; \quad A \text { and } \mathrm{J} \\
\text { in italics. }\end{array}$ \\
\hline 66 & 'SALTILTO' & $\begin{array}{l}\text { I } 7 \times 13 / 4 ; \text { between two } \\
\text { periods. }\end{array}$ \\
\hline & $\mathbf{Z A C}$ & I $6 \times 17 / 8$ \\
\hline
\end{tabular}

B. White wove paper, watermarked PAPEL SELLADO in the sheet. This watermark measures $158 \times 17 \mathrm{~mm}$, and runs vertically, covering eight stamps.
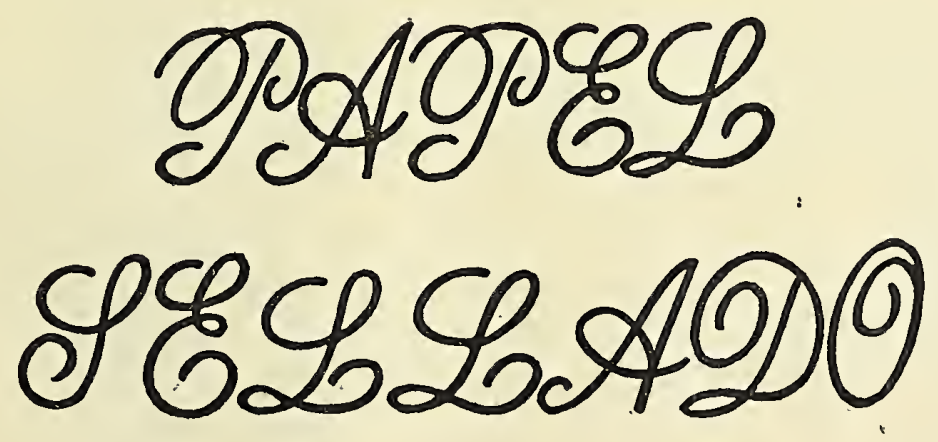
I. Unperforated.

I $^{\circ}$ Without any surcharge.

$12326 \mathrm{c}$ green

I233 I2c pale blue

I $23425 \mathrm{C}$ red

I235 $50 \mathrm{Oc}$ orange yellow

1236 IOOc gray violet

Variety: Error of color.

I237 50c blue

This stamp was never placed in actual use.

$2^{0}$ Surcharged with number and abbre viated date only.

I $2386 \mathrm{c}$ green

I239 I 2c pale blue

I240 $25 \mathrm{C}$ pale red

I24I 50c yellow

I242 IOOc gray violet

$3^{\circ}$ Surcharged with number and abbreviated date and name of postal district.

I243 6c green

I244 I $2 \mathrm{c}$ pale blue

I $24525 \mathrm{C}$ pale red

I $24650 \mathrm{oc}$ yellow

$12+7$ IOOC gray violet

\section{Pin Perforated.}

Surcharged with number and abbreviated date and name of postal district.

I $2486 \mathrm{c}$ green

I249 I 2c pale blue

I $250 \quad 25 \mathrm{C}$ pale red

I25 I $50 \mathrm{c}$ yellow

I252 IOOc gray violet

We do not think that the pin perforated stamps are ever found without any surcharge, or surcharged with number and date alone. All of the pin perforated stamps are only

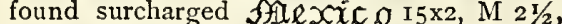
the only district in which they appear to have been used.

III. Square perforation.

$\mathrm{I}^{\circ}$ Surcharged with number and abbreviated date only.

I253 6c green

I254 I2c pale blue

I $25525 \mathrm{C}$ pale red

I256 50c yellow

I257 IOOc gray violet

Variety: Error of color.

Without any surcharge.

I258 $50 \mathrm{c}$ blue

$2^{\circ}$ Surcharged with number and abbreviated date and name of postal district.

$12596 \mathrm{c}$ green

I260 I2c pale blue

I26 I $25 \mathrm{C}$ pale red

I262 50c yellow

I263 Iooc gray violet

C. White wove paper, without watermark, varying slightly in thickness.

I. Unperforated.

$\mathrm{I}^{\circ}$ Without moiré pattern on back and without any surcharge.

I264 6c green

I265 I2c pale blue

I266 $25 \mathrm{c}$ pale red

I 267 50c yellow

I268 I00c gray violet

Most likely these are only essays.

$2^{\circ}$ With moiré pattern on back, without any surcharge.

I269 6c green

I270 6c pale green
127I 6c bluish green

I272 6c dark green

I273 I2c pale blue

I274 I2c dark blue

I $27525 \mathrm{c}$ pale red

I276 25c dark red

I277 50c orange

I278 50c yellow buff

I 279 50c yellow

I280 IoOc gray lilac

I28I IOOc gray violet

Varieties:

a. Error of color.

$\mathrm{r} 282 \quad 50 \mathrm{c}$ blue

b. Unperforated vertically.

I $283 \quad 25 \mathrm{c}$ red

c. Unperforated horizontally.

I 284 I $2 \mathrm{c}$ blue

$3^{\circ}$ Surcharged with number and abbreviated date only.

I284a 6c pale green

1285 6c yellow green

I $2866 \mathrm{c}$ bluish green

I 287 6c dark green

I 288 I $2 \mathrm{c}$ pale blue

I289 I2c dark blue

I $29025 \mathrm{C}$ pale red

I29I $25 \mathrm{C}$ dark red

I292 50c orange

I 293 5oc yel low buff

$129450 c$ yellow

I 295 Iooc gray lilac

I 296 Iooc gray violet

In 1888 a St. Louis firm obtained the original dies of these stamps, transfers of which were made in order to print from ; the stamps printed from these transfers are a trifle larger than the original stamps, measuring $191 / 2 \times 241 / 2$ $\mathrm{mm}$. instead of $191 / 2 \times 24 \mathrm{~mm}$. As the original paper with moiré pattern was not obtainable an imitation background was made consisting of vertical wavy lines, close together, with only a faint attempt at a moiré pattern, hardly perceptible. We cannot consider these stamps reprints, they being little better than counterfeits as the groundwork was forged. These so-called reprints exist also with forged surcharge of date, number and name of postal district.

$4^{\circ}$ Surcharged with number and abbreviated date and name of postal district.

1297 6c pale green

I298 6c yellow green

I 299 6c bluish green

I300 $6 \mathrm{c}$ dark green

I30I I2c pale blue

$\mathrm{I} 302$ I2c dárk blue

I $30325 \mathrm{C}$ pale red

$130425 \mathrm{c}$ dark red

I 305 50c orange

I306 50c yellow buff

I 307 50c yellow

I308 IOOC gray lilac

I309 IOOC gray violet

Stamps without surcharge, with number and date only are rarely found used.

II. Pin perforated 13 .

Surcharged with number and abbreviated date and name of postal district.

I3IO $6 \mathrm{c}$ pale green

I3I I 6c yellow green

I3I2 6c bluish green

I3I3 6c dark green

I3I4 I2c pale blue

I3I 5 I2c dark blue

I $31625 \mathrm{c}$ pale red 


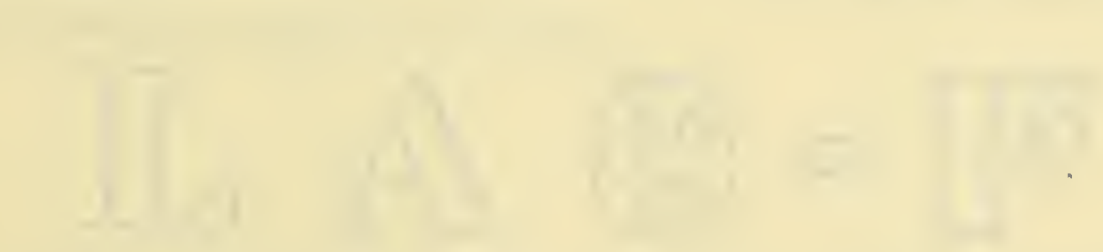





\section{I3I $7 \quad 25 \mathrm{C}$ dark red \\ I3I8 50c orange \\ I3I9 5Oc yellow buff \\ I320 $50 \mathrm{C}$ yellow \\ I32I IOOc gray lilac \\ I322 IOOC gray violet}

III. Pin perforated I4.

Surcharged with number and abbreviated date and name of postal district.

I323 6c pale green

I324 6c yellow green

I $3256 \mathrm{c}$ bluish green

1326 6c dark green

I327 I2c pale blue

I328 I2c dark blue

r $32925 \mathrm{c}$ pale red

I $330 \quad 25 \mathrm{c}$ dark red

r33I $50 c$ orange

1332 50c yellow buff

I333 5 Oc yellow

I334 IOOC gray lilac

I335 I00c gray violet

IV. Pin Perforated I5.

Surcharged with number and abbreviated date and name of postal district.

1336 6c pale green

I337 6c yellow green

$\mathrm{r} 3386 \mathrm{c}$ blue green

$13396 \mathrm{c}$ dark green

I340 I2c pale blue

r34I I2c dark blue

$134225 \mathrm{C}$ pale red

$134325 \mathrm{c}$ dark red

r344 5Oc orange

I345 5Oc yellow buff

13465 oc yellow

1347 IOOC gray lilac

I348 IOOc gray violet

The only surcharges known on the pin perforated stamps are: Mexico 15×2, M 21/2 and Mexico in curve IIXI $7 / 8$.

We do not believe that any of the pin perforated stamps exist without any surcharge, or with number and date alone.

V. Square perforation I5.

$\mathrm{I}^{\circ}$ Without any surcharge.

I $3496 \mathrm{c}$ pale green

I350 6c yellow green

I35 I 6c bluish green

1352 6c dark green

I353 I2c pale blue

\author{
I 354 I2c dark blue \\ I355 25c pale red \\ r $35625 \mathrm{C}$ dark red \\ r357 5Oc orange \\ I 358 50c yellow buff \\ I359 50c yellow \\ I360 rooc gray lilac \\ I36I IOOC gray violet \\ Variety: Error of color.
}

I362 50c blue

$2^{\circ}$ Surcharged with number and abbreviated date only.

I363 6c pale green

I364 6c yellow green

I365 6c bluish green

I 366 6c dark green

1367 I $2 \mathrm{c}$ pale blue

I368 I2c dark blue

I369 25c pale red

I 370 25c dark red

I37I $50 \mathrm{OC}$ orange

I 3725 Oc yellow buff

r373 50c yellow

I374 IOOc gray lilac

I375 rooc gray violet

$3^{\circ}$ Surcharged with number and abbreviated date and name of postal district.

$\begin{array}{rl}\text { I376 } & \text { 6c pale green } \\ \text { I377 } & \text { 6c yellow green } \\ \text { I378 } & 6 \mathrm{c} \text { bluish green } \\ \text { I } 379 & 6 \mathrm{c} \text { dark green } \\ \text { I380 } & \text { I2c pale blue } \\ \text { I38I } & \text { r2c dark blue } \\ \text { I382 } & \text { 25c pale red } \\ \text { I383 } & \text { 25c dark red } \\ \text { I384 } & \text { 50c orange } \\ \text { I385 } & \text { 50c yellow buff } \\ \text { I386 } & \text { 50c yellow } \\ \text { I387 } & \text { IOOc gray lilac } \\ \text { I388 } & \text { IOOc gray violet } \\ \text { VI. } & \text { Perforated in points I3. }\end{array}$

ananisaAinaAanana

Surcharged with number and abbreviated date and name of postal district.

r389 I2c dark blue

I 390 I $2 \mathrm{c}$ pale blue

I39I $25 \mathrm{C}$ pale red

I392 $25 \mathrm{c}$ dark red

The only stamps with this perforation seen by us were surcharged I Mexico 74.

On some of the sheets the following watermark is found:
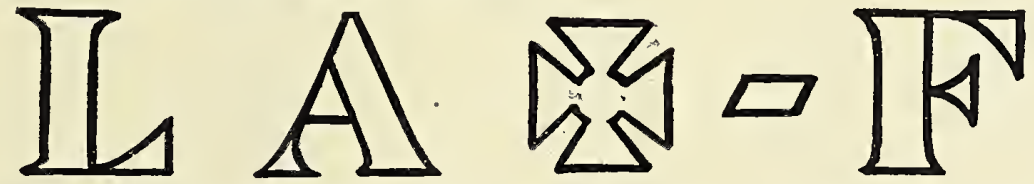

The cross in French is "CROIX", making the watermark read "I.A CROIX FRERES", manufacturers of this paper. This watermark has perplexed many a collector and compiler, no groups of these stamps having been found to show enough of the watermark to explain it; it was only lately that we were fortunate enough to find several blocks which enabled us to construct the watermark and thus explain the mystery.

Distric

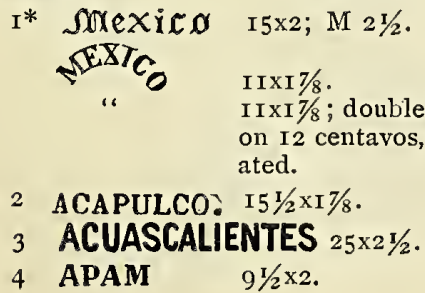
ated.

2 ACAPULCO: $151 / 2 \times 17 / 8$.

3 ACUASCALIENTES $25 \times 2 \frac{1}{2}$.

4 APAM

I I II $7 / 8$; double surcharge on 12 centavos, unperfor-
5* CAMPECHE

CAMPECHE

.I.DEL CARM EN. 2I 1/2xI 3/4; between

I7x2 ; double surcharge on the I 2 centavos. iwo periods.
$20 x I 3 / 4$; with one period.

CHACCO 


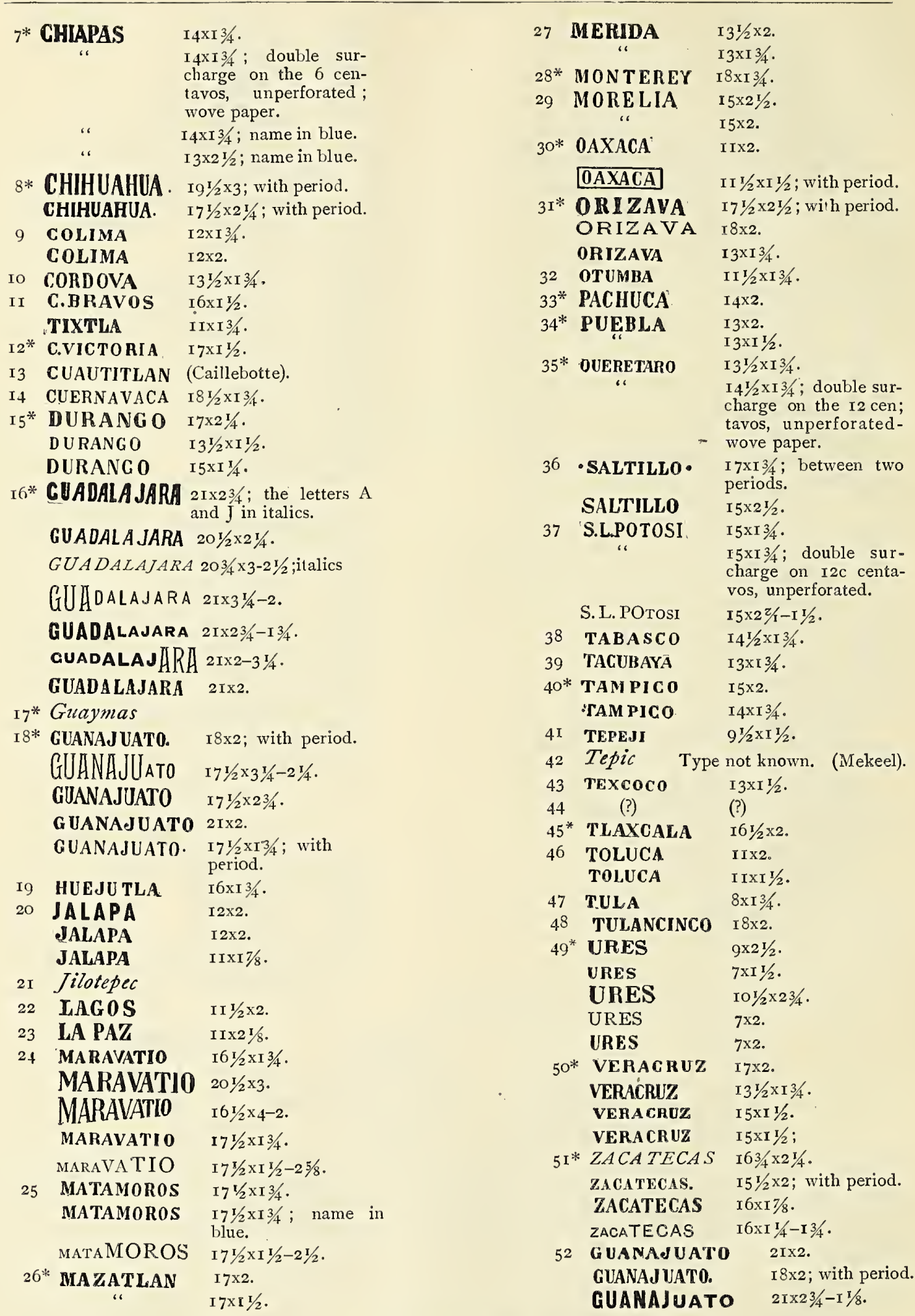

List of Surcharges we have seen on paper walermarked "PAPEL SEI.LADO".

\begin{tabular}{|c|c|c|c|c|}
\hline I Mexico & I $5 \times 2 ;$ M $21 / 2$. & & Tixtla & $\operatorname{IIXI} 3 / 4$ \\
\hline Mexico & I $1 \times 17 / 8$; in a curve & I 2 & C. Victoria & I $7 \times 1 \mathrm{I} / 2$ \\
\hline 2 Acapulco & $151 / 2 \times 17 / 8$. & I 5 & Durango & $131 / 2 \times 12$. \\
\hline Colima & $\mathrm{I} 2 \times \mathrm{X} 3 / 4$. & I6 & Guadalajara & \\
\hline
\end{tabular}





\begin{tabular}{|c|c|c|}
\hline 6 & Guadalajara & $201 / 2 \times 2 \pi / 4$. \\
\hline & Guadalajara & $2 \mathrm{IX} 2$. \\
\hline & No name & \\
\hline & Guanajuato & $171 / 2 \times 23 / 4$. \\
\hline & Jalapa & $\operatorname{IIXI} 7 / 8$. \\
\hline & Lagos & I I $1 / 2 \times 2$. \\
\hline & Matamoros & I $71 / 2 \times 13 / 4$. \\
\hline & Merida & $131 / 2 \times 2$ \\
\hline & Monterey & I $8 \times$ I $3 / 4$. \\
\hline & Morelia & $15 \times 21 / 2$ \\
\hline & Orizava & I $3 \times 13 /$ \\
\hline
\end{tabular}

\begin{tabular}{|c|c|c|}
\hline 34 & Puebla & $13 \times 2$. \\
\hline 35 & Queretaro & I $41 / 2 \times$ I $3 / 4$. \\
\hline 36 & Saltillo & I $7 \times 13 / 4 ;$ between periods. \\
\hline 36 & Saltillo & $15 \times 21 / 2$ \\
\hline 37 & S. L. Potosi & $15 \times 13 / 4$. \\
\hline 40 & Tampico & I $5 \times 2$ \\
\hline 44 & No name & \\
\hline 46 & Toluca & I IX2. \\
\hline 50 & Vera Cruz & $141 / 2 \times 11 / 2$ \\
\hline & Zacatecas & I $6 \times 17 / 8$. \\
\hline
\end{tabular}

ERRORS.

Surcharged with name of postal district only.

Ures 9x2 (Heitman's Handbuch.)

Surcharged with wrong numbers.

\begin{tabular}{|c|c|c|c|}
\hline $\begin{array}{l}\text { District. } \\
\text { Mexico }\end{array}$ & $\begin{array}{l}\text { Value. } \\
\text { I 2C }\end{array}$ & No. & $\begin{array}{r}\text { Date. } \\
72\end{array}$ \\
\hline Cordova & $25 \mathrm{c}$ & $3 I$ & 73 \\
\hline Guanajuato & $25 \mathrm{c}$ & I6 & 72 \\
\hline Maravatio & I $2 \mathrm{C}$ & 42 & 73 \\
\hline Merida & I $2 \mathrm{C}$ & 5 & 74 \\
\hline Monterey & $25 \mathrm{c}$ & 2 & 73 \\
\hline Morelia & I $2 \mathrm{C}$ & 3 I & 72 \\
\hline ." & $25 \mathrm{c}$ & $3 I$ & 72 \\
\hline Orizava & I $2 \mathrm{c}$ & 29 & 72 \\
\hline “ & $25 \mathrm{c}$ & 29 & 72 \\
\hline "6 & $25 \mathrm{c}$ & 29 & 72 \\
\hline$" 6$ & 500 & 29 & 72 \\
\hline “ & I $2 \mathrm{C}$ & 29 & 72 \\
\hline “ & I2C & 29 & $?$ \\
\hline Puebla & I2C & 43 & 73 \\
\hline Saltillo & $6 c$ & 3 & $?$ \\
\hline Tabasco & I $2 \mathrm{C}$ & 7 & 72 \\
\hline Ures & I2C & 94 & 73 \\
\hline Vera Cruz & $?$ & 05 & $?$ \\
\hline Zacatecas & I $2 \mathrm{C}$ & I5 & 73 \\
\hline$“$ & I $2 \mathrm{C}$ & OI & 73 \\
\hline “ & I2C & OI & 73 \\
\hline $\begin{array}{l}\text { Campeche } \\
\text { Errors in dating. }\end{array}$ & I $2 \mathrm{C}$ & 27 & 74 \\
\hline $\begin{array}{l}\text { District. } \\
\text { Toluca }\end{array}$ & $\begin{array}{l}\text { Value. } \\
\text { I2c }\end{array}$ & $\begin{array}{l}\text { No. } \\
46\end{array}$ & $\begin{array}{r}\text { Date. } \\
47\end{array}$ \\
\hline Mexico & I $2 \mathrm{C}$ & I & 4 \\
\hline
\end{tabular}

\begin{tabular}{|c|c|c|c|c|c|}
\hline $\begin{array}{l}\text { Perforation. } \\
\text { Unperf. }\end{array}$ & $\begin{array}{l}\text { Meas. of Sur. } \\
\text { ? }\end{array}$ & instead of & I & 72 & \\
\hline & I $4 \times 13 / 4$ & $" 6$ & IO & 73 & \\
\hline " & $?$ & “ & I 8 & 72 & Papel Sellado \\
\hline$"$ & $161 / 2 \times 11 / 2$ & “ & 24 & 73 & \\
\hline "، & $131 / 2 \times 2$ & “6 & 27 & 74 & \\
\hline “ & I $8 \times 13 / 4$ & "6 & 28 & 73 & \\
\hline " & $15 \times 2$ & “ & 29 & 72 & \\
\hline 6 & I $5 \times 2$ & “' & 29 & 72 & \\
\hline “ & I $3 \times 13 / 4$ & “ & $3 I$ & 72 & \\
\hline “ & I $3 \times 13 / 4$ & “ & $3 I$ & 72 & \\
\hline “ & I $3 \times 13 / 4$ & “ & $3 I$ & 72 & Papel Sellado \\
\hline “ & I $3 \times 13 / 4$ & “ & $3 I$ & 72 & \\
\hline “ & $I 7 \times 21 / 4$ & " & $3 I$ & 72 & \\
\hline Sq. Perf. & $?$ & $" 6$ & 31 & $?$ & \\
\hline Ủnperf. & $13 \times 2$ & $"$ " & 34 & 73 & \\
\hline$\because$ & $?$ & “ & 36 & $?$ & \\
\hline “ & $141 / 2 \times 1 / 2$ & “ & 38 & 72 & \\
\hline “6 & $7 \times 2$ & “ & 49 & 73 & \\
\hline$?$ & $?$ & “ & 50 & $?$ & \\
\hline Unperf. & I $6 \times 13 / 4$ & "، & $5 \mathrm{I}$ & 73 & \\
\hline$" 6$ & I $6 \times 13 / 4$ & "“ & $5 \mathrm{I}$ & 73 & \\
\hline$" 6$ & I $6 \times 13 / 4$ & " & $5 \mathrm{I}$ & 73 & \\
\hline " & $17 \times 2$ & ““ & 5 & 74 & \\
\hline
\end{tabular}

Errors in position of number and date, thus:

Perforation. Meas. of Sur.

Unperf. IIXI $1 / 2$ instead of $46 \quad 74$

Sq. Perf. IIXI $7 / 8$ " I 74

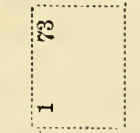

$\begin{array}{lrrrccc}\begin{array}{l}\text { District. } \\ \text { Mexico }\end{array} & \text { Value. } & \text { No. } & \text { Date. } & \begin{array}{c}\text { Perforation. } \\ \text { Sq. Perf. }\end{array} & \begin{array}{c}\text { Meas. of Sur. } \\ \text { I IXI } 7 / 8\end{array} \\ \text { Campeche } & \text { 2c } & \text { I } & 73 & \begin{array}{c}\text { Squ } \\ \text { Unperf. }\end{array} & \text { I7 } 7 \times 2 & \text { This is the error of number 27 } \\ \text { instead of } 5 .\end{array}$

Number and date, thus:

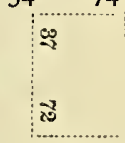

District. Value. No. Date. Perforation. Meas. of Sur.

(S. L. Potosi) I2c $\quad 37 \quad 72 \quad$ Unperf. Not Surcharged.

Number and date, thus: $\quad{ }_{-}$

\begin{tabular}{|c|c|c|c|c|c|c|}
\hline $\begin{array}{c}\text { District. } \\
\text { Guanajuato }\end{array}$ & $\begin{array}{l}\text { Value. } \\
\text { I2C }\end{array}$ & $\begin{array}{c}\text { No. } \\
73\end{array}$ & $\begin{array}{r}\text { Date. } \\
\text { I } 8\end{array}$ & $\begin{array}{l}\text { Perforation. } \\
\text { Unperf. }\end{array}$ & $\begin{array}{l}\text { of Sur. } \\
\text { given in Iteit }\end{array}$ & \\
\hline \multicolumn{7}{|c|}{ Names of Postal Districts found surcharged twice. } \\
\hline $\begin{array}{l}\text { Value. } \\
\text { I2c }\end{array}$ & $\begin{array}{r}\text { Distr } \\
\text { Mexic }\end{array}$ & & I & $\begin{array}{l}\text { Measure of Surcharge. } \\
\text { I IXI } 7 / 8\end{array}$ & $\begin{array}{l}\text { Perforation. } \\
\text { Unperforated }\end{array}$ & $\begin{array}{l}\text { Paper. } \\
\text { Wove }\end{array}$ \\
\hline I $2 \mathrm{C}$ & Camp & che & 5 & $\begin{array}{l}17 \times 2 \\
I 72\end{array}$ & “" & " \\
\hline $6 c$ & Chiap & & 7 & I $4 \times 53 / 4$ & ، & .. \\
\hline I $2 \mathrm{C}$ & Quere & aro & 35 & $131 / 2 \times 13 / 4$ & “ & " \\
\hline I $2 \mathrm{c}$ & S. L. & Potosi & 37 & $15 \times 134$ & " & \\
\hline
\end{tabular}


Errors in perforation.

I. Square perforation.

$I^{0}$ Double perforation vertically.

\begin{tabular}{|c|c|c|c|c|}
\hline | District. & Value. & No. & Date. & Meas. of Sur. \\
\hline Chiapas & $12 \mathrm{c}$ & 7 & 73 & I $4 \times 13 / 4$ in blue. \\
\hline C. Victoria & $6 c$ & 12 & 72 & $17 \times I 1 / 2$ \\
\hline Durango & $12 \mathrm{c}$ & I5 & 73 & I $31 / 2 \times 11 / 2$ \\
\hline (Guaymas) & I $2 \mathrm{c}$ & I 7 & 72 & \\
\hline Mazatlan & I2c & 26 & 74 & $17 \times 2$ \\
\hline Orizava & $25 \mathrm{c}$ & $3 \mathrm{I}$ & 74 & $18 \times 2$ \\
\hline (Orizava) & I $2 \mathrm{c}$ & $3 I$ & 73 & \\
\hline (Pachuca) & I $2 \mathrm{c}$ & 33 & 73 & \\
\hline (Pachuca) & $25 \mathrm{c}$ & 33 & 73 & \\
\hline Pachuca & $\begin{array}{l}12 \mathrm{c} \\
50 \mathrm{c}\end{array}$ & $\begin{array}{c}33 \\
\text { none }\end{array}$ & 73 & $\begin{array}{l}\text { I } 4 \times 2 \\
\text { No Surcharge }\end{array}$ \\
\hline
\end{tabular}

$2^{\circ}$ Double perforation horizontally.

\begin{tabular}{|c|c|c|c|c|}
\hline District. & Value. & No. & Date. & Meas. of Sur \\
\hline Guanajuato & I2C & 18 & 73 & \\
\hline Monterey & IOOC & 28 & 72 & I $8 \times 13 / 4$ \\
\hline Monterey & $12 \mathrm{c}$ & 28 & 73 & I $8 \times 13 / 4$ \\
\hline Orizava & $25 \mathrm{c}$ & $3 I$ & 73 & $18 \times 2$ \\
\hline (Orizava) & I $2 \mathrm{c}$ & $3 I$ & 73 & \\
\hline Pachuca & I $2 \mathrm{C}$ & 33 & 73 & $14 \times 2$ \\
\hline (Pachuca) & I $2 \mathrm{c}$ & 33 & 73 & \\
\hline
\end{tabular}

$3^{\circ}$ Double perforation horizontally and vertically.

$\begin{array}{lcrrc}\text { District. } & \text { Value. } & \text { No. } & \text { Date. } & \text { Meas. of Sur. } \\ \text { Orizava } & \text { 25c } & 3 \mathbf{I} & 73 & \mathbf{I} 8 \times \mathbf{2} \\ \text { Pachuca } & \text { I2c } & 33 & 73 & \mathbf{I 4 \times 2}\end{array}$

II. Pin perforation.

District.

Mexico

Mexico

District.

Mexico

May, 1874 .

Head of Hidalgo engraved by the American Bank Note Company of New York, and printed on paper of various kinds.
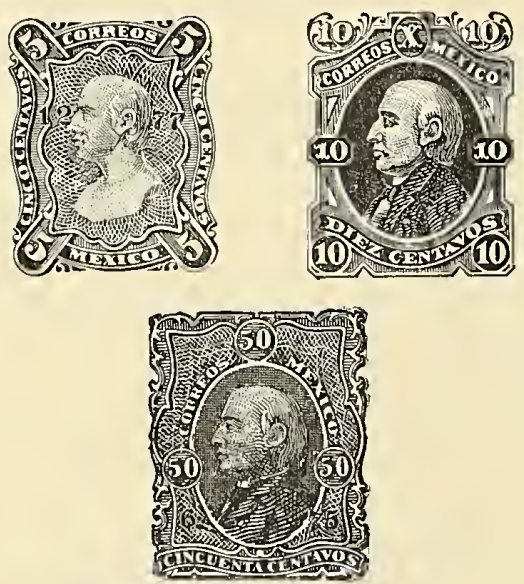

A. Unwaterinarked paper.

Number and date far apart,

$$
1 \quad 79
$$

I. Thin crisp wove paper.

Perforated $\mathbf{2}$.

$\begin{array}{cc}\text { No. } & \text { Date. } \\ \text { I } & 72 \\ \text { I } & 72\end{array}$

$$
\text { No. Date. }
$$

$I^{\circ}$ Double perforation vertically.

\section{Meas, of Sur.}

IIXI7/8 (pin perforated I2, I3 and I4). $2^{\circ}$ Double perforation horizontally.$$
15 \times 2 ; \mathrm{M} 21 / 2^{1} \text { (pin perforated } 14 \text { ). }
$$

Meas, of Sur.

I5X2; M 2I/2 (pin perforated I2, I3 I4).

$I^{0}$ Surcharged with number and abbre. viated only.

I393 5c pale brown

I 394 5 dark brown

I395 5c yellow brown

I $3965 \mathrm{C}$ chestnut brown

I397 roc black

I398 25c blue

I399 25c dark blue

I400 $25 \mathrm{c}$ dull light blue

I 40 I $50 \mathrm{c}$ dark green

I 402 50c yellow green

I403 Iopc carmine

$2^{\circ}$ Surcharged with number and abbreviated date and name of postal district.

I404 $5 \mathrm{c}$ pale brown

I405 5c dark brown

I $406 \quad 5 \mathrm{c}$ yellow brown

I 407 5C chestnut brown

I 408 Ioc black

I $40925 \mathrm{c}$ blue

I4IO $25 \mathrm{c}$ dark blue

I4I I $25 \mathrm{c}$ dull light blue

I4I2 50c dark green

I4I3 50c yellow green

I 4 I 4 IOOc carmine

II. On thick wove paper.

Perforated I2.

$I^{\circ}$ Surcharged with number and abbreviated date only.

I4I5 $5 \mathrm{c}$ pale brown

I4I6 5c dark brown 




I4I7 $5 \mathrm{c}$ yellow brown
I4I8 $5 \mathrm{c}$ chestnut brown
I4I9 IOc black
I420 $25 \mathrm{c}$ blue
I42I $25 \mathrm{c}$ dull.light blue
I422 $50 \mathrm{c}$ dark green
I423 $50 \mathrm{c}$ yellow green
1424 IOOc carmine
$2^{\circ}$ Surcharged with number and abbre-

viated date and name of postal district.

\begin{tabular}{|c|c|}
\hline 425 & 5c pale brown \\
\hline$[42$ & $5 c$ dark brown \\
\hline 1427 & 5 c yellow brown \\
\hline & $5 \mathrm{c}$ chestnut brow \\
\hline & Ioc black \\
\hline & $25 \mathrm{c} \mathrm{blu}$ \\
\hline I 431 & $25 \mathrm{c}$ dull light blue \\
\hline & 5oc dark green \\
\hline & $50 c$ yellow green \\
\hline & $T$ \\
\hline
\end{tabular}

III. On ordinary medium wove paper.

$\mathrm{I}^{\circ}$ Without surcharge.

I 435 5c brown
I 436 IOc black
I 437 25c blue
I 438 50c green
I 439 IOOc carmine
Varieties: Unperforated.

$1440 \quad 25 \mathrm{c}$ blue

I 44 I IOOc carmine

We have never seen any used specimens without surcharge.

$2^{\circ}$ Surcharged with number and abbreviated date only.

\begin{tabular}{|c|c|}
\hline I 442 & 5c pale brown \\
\hline I 443 & $5 \mathrm{c}$ dark brown \\
\hline I 444 & 5 c yellow brown \\
\hline 1445 & $5 \mathrm{c}$ chestnut brown \\
\hline 1446 & IOc black \\
\hline 1447 & $25 \mathrm{c}$ blue \\
\hline I 448 & $25 \mathrm{c}$ dark blue \\
\hline I 449 & $25 \mathrm{c}$ dull light blue \\
\hline r 450 & 50c dark green \\
\hline I $45 \mathrm{I}$ & $50 c$ yellow green \\
\hline 1452 & IOOc carmine \\
\hline
\end{tabular}

$3^{\circ}$ Surcharged with number and abbreviated date and name of postal district.

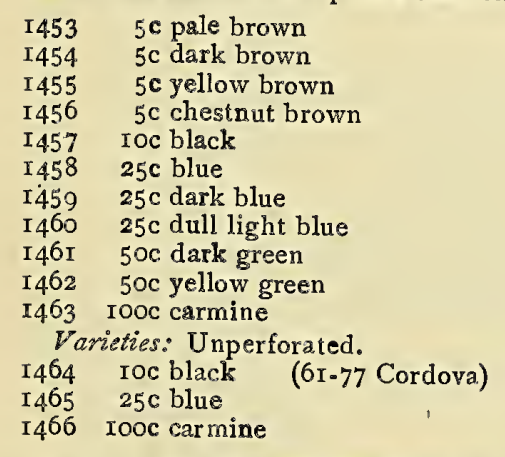

IV. On medium laid paper, lines vertical and close together.

Perforated I2.

$I^{\circ}$ Without surcharge.

I 467 5 5 brown

$146825 \mathrm{c}$ blue

1469 rooc carmine

We have never seen any used specimens without surcharge.

$2^{\circ}$ Surcharged with number and abbre. viated date only.
I470 $5 \mathrm{c}$ yellow brown

I47I $5 \mathrm{c}$ dark brown

1472 IOc black

I473 roc intense black

I474 25c dark blue

I 475 50c yellow green

I 476 IO0c carmine

$3^{\circ}$ Surcharged with number and abbreviated date and name of postal district.

I $4775 \mathrm{c}$ yellow brown

I 478 5 dark brown

I 479 IOc black

I480 IOc intense black

I 48 I $25 \mathrm{c}$ blue

I 482 25c dark blue

I483 50c yellow green

I 484 IOOc carmine

$\mathrm{V}$. On medium laid paper, lines vertical and close together, ribbed horizontally.

Perforated 12.

$I^{\circ}$ Surcharged with number and abbreviated date only.

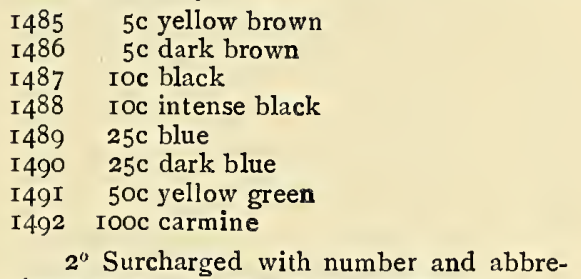
viated date and name of postal district.

I493 5c yellow brown

I $4945 \mathrm{c}$ dark brown

I495 IOc black

I $49625 \mathrm{c}$ blue

I 497 25c dark blue

I498 50c yellow green

I499 Iooc carmine

VI. On thick laid paper, lines vertical and close together.

Perforated I2.

$I^{\circ}$ Surcharged with number and abbreviated date only.

I $500 \quad 5 \mathrm{c}$ yellow brown

I5OI 5c dark brown

I 502 IOc black

I 503 IOc intense black

I $50425 \mathrm{c}$ blue

I505 25c dark blue

I506 5Oc yellow green

I507 IOOc carmine

$2^{\circ}$ Surcharged with number and abbreviated date and name of postal district.

I508 $5 \mathrm{C}$ yellow brown

I $5095 \mathrm{c}$ dark brown

I. 5 IO IOc black

I5 I I IOc intense black

I 5 I $25 \mathrm{c}$ blue

I5I3 25c dark blue

I5I4 50c yellow green

I5I5 IOOc carmine

VII. On medium laid paper, lines horizontal and close together.

Perforated I2.

$\mathbf{I}^{\circ}$ Surcharged with number and abbreviated date only. I5 I6 $25 \mathrm{c}$ pale dull blue

$2^{\circ}$ Surcharged with number and abbreviated date and name of postal district.

I5I $725 \mathrm{c}$ pale dull blue 
VIII. On medium laid paper, lines horizontal and very broad.

Perforated I2.

$I^{\circ}$ Surcharged with number and abbreviated date only.

I5I 8 IOc intense black

I5I9 $25 \mathrm{C}$ dark blue

$2^{\circ}$ Surcharged with number and abbreviated date and name of postal district.

I520 Ioc intense black

I 52 I $25 \mathrm{c}$ dark blue

IX. On medium wove paper, having quadrille appearance.

Perforated I2.

$\mathrm{I}^{\circ}$ Surcharged with number and abbreviated date only.

I522 $5 \mathrm{c}$ brown

1523 IOc black

I524 25c pale dull blue

I525 $25 \mathrm{c}$ blue

I 526 50c moss green

1527 IOOC carmine

$2^{\circ}$ Surcharged with number and abbreviated date and name of postal district.

I528 $5 \mathrm{c}$ brown

I529 IOc black

I $530 \quad 25 \mathrm{c}$ pale dull blue

I53I $25 \mathrm{c}$ blue

I 532 5Oc moss green

I533 IOOc carmine

B. Paper Watermarked PAPEL SEL$\mathrm{LADO}$ as preceding issue.

I. Medium wove paper, having quadrille appearance.

Perforated 12.

$I^{\circ}$ Surcharged with number and abbreviated date only.

I 534 5 brown
I535 IOc black

I $53625 \mathrm{c}$ pale dull blue

I537 $25 \mathrm{c}$ blue

I 538 50c moss green

I539 IOOc carmine

$2^{\circ}$ Surcharged with number and abbreviated date and name of postal district.

I540 $5 \mathrm{c}$ brown

I 54 I IOc black

I $54225 \mathrm{c}$ pale dull blue

I $54325 \mathrm{c}$ blue

I $54450 \mathrm{c}$ moss green

I545 IOOC carmine

II. Medium laid paper, lines vertical and close together.

Perforated I2.

$I^{0}$ Surcharged with number and abbreviated date only.

I546 5c yellow brown

I547 $5 \mathrm{c}$ dark brown

I548 IOc intense black

I $54925 \mathrm{c}$ blue

I550 25c dark blue

I55I 50c yellow green

I552 IOOC carmine

$2^{\circ}$ Surcharged with number and abbreviated date and name of postal district.

I553 $5 \mathrm{c}$ yellow brown

I $554 \quad 5 \mathrm{c}$ dark brown

I 555 IOc intense black

$155625 \mathrm{c}$ blue

I557 25c dark blue

I 558 5Oc yellow green
I 559 IOOc carmine

$3^{\circ}$ Medium laid paper, lines horizontal and close together.

I $560 \quad 25 \mathrm{c}$ blue

Names of postal districts found surcharged on this issue.

I $N I^{\prime} C_{O}$ IIX2\%

SiXTC IIXI7/8.

2 ACAPULCO, $15 \% \frac{1}{2} \times 17 / 8$.

3 ACUASCALIENTES $25 \times 21 / 2$. AGUASCALIENTES $23 \frac{1}{2} \times 2 \frac{1}{4}$.

AGUASCALIENTES $25 \times 21 / 2-13 / 4$.

4 APAM $91 / 2 \times 2$.

" $\quad 9 \frac{1}{2} \times 2 ;$ name in blue.

5 CHMPECHE $141 \frac{1}{2} \times 3$.

CAMP ECHE I $\quad$ 7 $\times 2$.

.I.DEL CARM EN. 21 1 $1 / 2 \times 13 / 4$;

between two periods.

6 CHAECO $\operatorname{IIX} I 3 / 4$.

CHALCO $12 \frac{1}{2} \times 17 / 8$

CHALCO $13 \times 21 / 2$.

7 CHIAPAS $14 \times 13 / 4 ;$ name in blue.

8 CHIHUAHUA. I $7 \frac{1}{2} \times 21 / 4$; with period.

GHIHUAHUA. I9 $1 / 2 \times 3$; with period. CHIHUAHUA $18 \frac{1}{4} \times 2 \times 2 / 2-3$.

9 COLIMA $131 / 2 \times 2 \frac{1}{4}$.

". $13 \frac{1}{2} \times 2 \frac{1}{4}$; name in

blue.

COLIMA I $3 \times 13 / 4$.

IO

$\left.\begin{array}{ll}\text { “. } & \text { I } 33 / 4 \times 2 . \\ & \text { name in blue. }\end{array}\right\} \begin{aligned} & \text { These are } \\ & \text { really the } \\ & \text { same } \\ & \text { surcharge }\end{aligned}$

C.BRAVOS I $6 \times 13 / 4$.

“ - $16 \times 13 / 4$; name in blue.

$16 \times 1 \frac{1}{2}$.

I2

C.VICTORIA I $7 \times I \frac{1}{2}$.

“ $17 \times 1 \frac{1}{2}$; name in violet.

I3 CUAUTITLAN I $81 / 2 \times 2$.

I4 CUERNAVACA $181 / 2 \times 13 / 4$.

I DURANG O. I8 $3 / 4 \times 2$; with period. DURANGO I $4 \times 13 / 4$.

I6

CUADALAJARA $21 \times 23 / 4$.

GUADALAJARA $21 \times 2 \frac{12}{2}-3$.

CUADALAJARA $23 \times 23 \%$.

“ 23×23/4; name in blue

GUADALAJARA $181 / 2 \times 17 / 8$.

“ $181 / 2 \times 17 / 8$; name in

blue.

I $81 / 2 \times 11 / 2$.

GUADALAJARA I $7 \frac{1}{2} \times 1 \frac{1}{2} ;$ name in black.

“ $\quad 17 \frac{1}{2} \times 1 \frac{1}{2}$; name in blue.

$\left.\begin{array}{l}\text { I7 Guaymas (?) } \\ \text { I Guanajuato }\end{array}\right\}$ Never been found.

I9 HUEJUTLA I6xi $3 / 4$.

I6xI 3/4; name in blue. 
1 n 


\section{JALAPA \\ JALAPA \\ 2I Jilotepec \\ 22 LAG OS \\ 23 LA PAZ \\ 24 MARAVATIO}

MARAVATIO MARAVATIO

MARAVATIO

MATAMOROS $22 \times 3$.

MATAMOROS I $7 \frac{1}{2} 2 \times 13 / 4$.

$17 \frac{1}{2} \times 13 / 4 ;$ name in

blue.

MATAMOROS $171 / 2 \times 11 / 2-2$.

26 MAZATLAN $17 \times 2$.

MAZATLAN $17 \times 1 \mathrm{I} / 2$.

27 MERIDA $13 \times 3$

MERIDA $13 \times 2$.

“ 13XI3/4; name in

MONTEREY I $8 \times 2$.

-MONTERREY. I9 $12 \times 2 \times 17 / 8$; between

two periods, vermilion surcharge.

MONTEREI I9 $1 / 2 \times 2$.

MONTERREY I $9 \times 2$.

29 MORELIA $\mathrm{I}_{4} \times 2$.

MORELIA I5 2 .

MORELIA I $6 \times 21 / 4$.

30 OAXACA. I6 2 ; with period.

OAXACA II $1 / 2 \times 13 / 4$.

$\mathrm{II} 1 / 2 \times 13 / 4 ;$ name in

blue.

\begin{tabular}{ll} 
OAXACA & IIX2; name in blue. \\
\hline 1 & IIX2; name in black.
\end{tabular}

I

ORIZAVA

$17 \times 23 / 4$, name in

blue.

$17 \times 23 / 4$; name in

reddish violet.

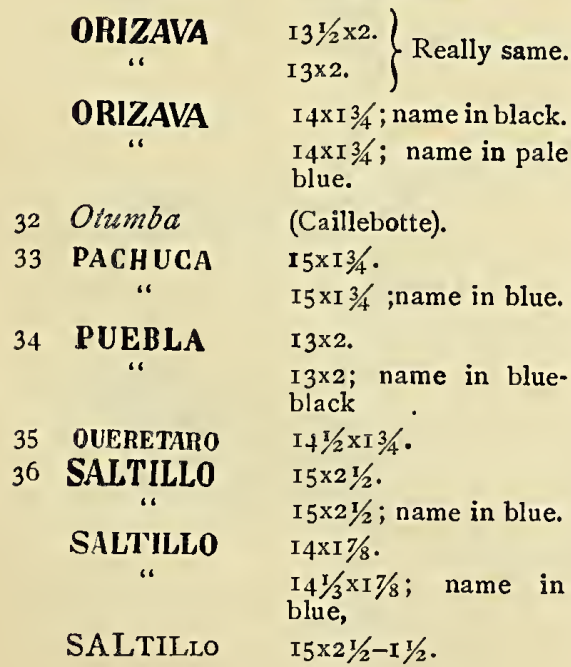

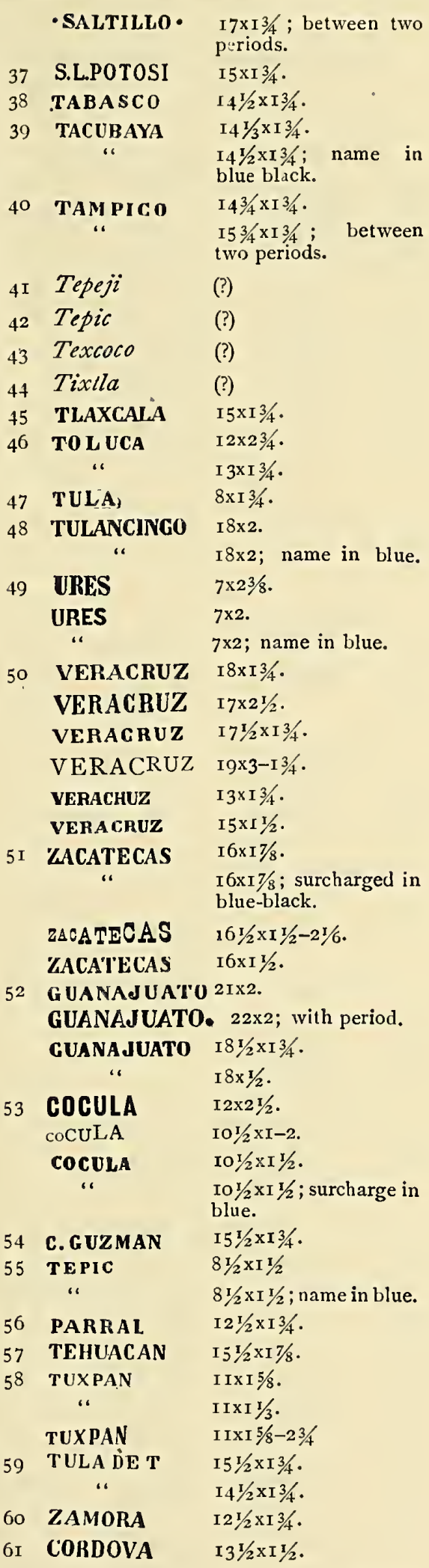

Almost all of the numbers can be found surcharged with number and abbreviated date only. 


\section{HABILITADO}

5 cent thin crisp wove paper surcharged Toluca in black, and Ixtlahuaca in blue. Date 46 , '74.

\section{ERRORS.}

Errors in numbers.

\begin{tabular}{|c|c|c|c|c|c|c|c|}
\hline Value. & District. & \multicolumn{2}{|c|}{ No. Date. } & \multicolumn{4}{|c|}{ Meas. of Sur. } \\
\hline $25 \mathrm{c}$ & Maravatio & 20 & ? & instead of & 24 & ? & ? \\
\hline $25 \mathrm{c}$ & Mazatlan & 2 & 77 & “ & 26 & 77 & $17 \times 2$ \\
\hline $25 \mathrm{C}$ & Merida & 5 & 76 & “" & 27 & 76 & $\begin{array}{l}\text { I3XI 3/4 } \\
\text { blue black }\end{array}$ \\
\hline $25 \mathrm{c}$ & Morelia & 25 & ? & “ & 29 & ? & $?$ \\
\hline IOC & " & 25 & 76 & 16 & 29 & 76 & I $5 \times 2$ \\
\hline IOOC & Orzava & I3 & $?$ & “" & $3 I$ & $?$ & ? \\
\hline $25 \mathrm{c}$ & Puiebla & $3 I$ & 75 & " & 34 & 75 & I $3 \times 2$ \\
\hline $25 \mathrm{c}$ & $" 6$ & 3 I & 77 & $"$ & 34 & 77 & $13 \times 2$ \\
\hline $25 \mathrm{c}$ & Toluca & 6 & 74 & $“$ & 46 & 74 & I $3 \times I 3 / 4$ \\
\hline IOC & Vera Cruz & 59 & 75 & $"$ & 50 & 75 & I $71 / 2 \times 13 / 4$ \\
\hline $25 \mathrm{C}$ & ““ & 59 & $?$ & $"$ & 50 & $?$ & $?$ \\
\hline IOC & “" & 90 & ? & “" & 5 & $?$ & $?$ \\
\hline $5 \mathrm{C}$ & Zacatecas & I2 & 77 & “ & $5 I$ & 77 & I $6 \times 17 / 8$ \\
\hline $5 \mathrm{c}$ & $" “$ & I5 & 77 & $\because$ & $5 I$ & 77 & None \\
\hline $25 \mathrm{c}$ & “" & 15 & ? & $“$ & $5 \mathrm{I}$ & $?$ & ? \\
\hline $5 \mathrm{c}$ & rdova & OI & ? & $" “$ & $6 I$ & ? & ? \\
\hline
\end{tabular}

Errors in date.

\begin{tabular}{|c|c|c|c|c|c|c|}
\hline Value. & District. & No. Date. & & & & eas. of Sur. \\
\hline IC & Mexico & I 67 & instead of & I & 76 & ? \\
\hline $25 \mathrm{c}$ & “ & I 67 & “ & I & 76 & I IXI $7 / 8$ \\
\hline IOC & " & 7 & ، & I & 7? & $\operatorname{IIXI} 7 / 8$ \\
\hline
\end{tabular}

With number only.

\begin{tabular}{|c|c|c|c|}
\hline $\begin{array}{l}\text { Value. } \\
25 \mathrm{C}\end{array}$ & $\begin{array}{l}\text { District. } \\
\text { Mazatlan }\end{array}$ & $\begin{array}{l}\text { No. } \\
26 \text { No date. }\end{array}$ & $\begin{array}{l}\text { Meas. of Sur. } \\
\quad \text { I } 7 \times 2\end{array}$ \\
\hline $25 c$ & S. L. Potosi & 37 & I $5 \times$ I $3 / 4$ \\
\hline
\end{tabular}

With date only.

\begin{tabular}{|c|c|c|c|c|}
\hline $\begin{array}{c}\text { Value. } \\
5 \mathrm{c}\end{array}$ & $\begin{array}{l}\text { District. } \\
\text { Oaxaca }\end{array}$ & No number & $\begin{array}{c}\text { Date. } \\
74\end{array}$ & $\begin{array}{l}\text { Meas. of Sur. } \\
\text { I I } 1 / 2 \times 1 \text { I } 3 / 4 \\
\text { framed }\end{array}$ \\
\hline
\end{tabular}

With name of postal district only.

\begin{tabular}{|c|c|c|}
\hline Value. & District. & Meas. of Sur. \\
\hline $5 \mathrm{c}$ & Mexico & I IXI $7 / 8$ \\
\hline $2 \mathrm{c}$ & Puebla & $13 \times 2$ \\
\hline $2 \mathrm{c}$ & S. L. Potosi & $15 \times 13 / 4$ \\
\hline
\end{tabular}

With number and date inverted.

$\begin{array}{ccclc}\text { Value. } & \text { District. } & \text { No. Date. } & \text { Meas. of Sur. } & \text { Paper. } \\ \text { 2c } & \text { Vera Cruz } & 576 & 76 \text { 3 } 3 / 4 & \text { Thick wove. }\end{array}$

With number and date placed wrong.

\begin{tabular}{|c|c|c|c|c|c|c|c|}
\hline Value. & District. & \multicolumn{2}{|c|}{ No. Date. } & & & \multirow{3}{*}{$\begin{array}{l}\text { Meas. of Sur. } \\
\text { No sur. }\end{array}$} & \multirow{4}{*}{$\begin{array}{l}\text { Paper. } \\
\text { Wove, apparently quadrille. } \\
\text { Wove, apparently quadrille, } \\
\text { w'r'm'k Papel Sellado. }\end{array}$} \\
\hline IOoc & Durango & 77 & I5 & instead of 15 & 77 & & \\
\hline IOOC & & 77 & 15 & 15 & 77 & & \\
\hline $25 \mathrm{c}$ & Orizava & 74 & $3 I$ & $3 I$ & 74 & $13 \times 2$ & \\
\hline $25 \mathrm{c}$ & & 77 & $3 \mathrm{I}$ & $3 \mathrm{I}$ & 77 & $\begin{array}{r}17 \times 23 / 4 \\
\text { red violet }\end{array}$ & Ordinary med. wove. \\
\hline IOC & Puebla & 77 & 34 & 34 & 77 & $13 \times 2$ & Med. vert. laid. \\
\hline $5 \mathrm{c}$ & Vera Cruz & 77 & 50 & 50 & 77 & $?$ & 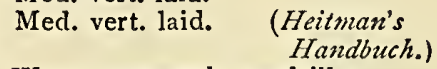 \\
\hline IOOC & Guanajuato & 77 & 52 & 52 & 77 & No sur. & Wove, apparently quadrille, \\
\hline IOOC & & 77 & 52 & 52 & 77 & & $\begin{array}{l}\text { Wove, apparently quadrille, } \\
\text { w'r'm'k Papel Sellado. }\end{array}$ \\
\hline $50 \mathrm{c}$ & La Paz & & 237 & 23 & 74 & I IX2 $1 / 8$ & Thin crisp wove. \\
\hline
\end{tabular}

Two stamps connected, one surcharged with name of town, the other not.

$\begin{array}{cllcc}\text { Value. } & \text { District. } & \text { No. Date } & \text { Meaf. of Sur. } & \text { Paper. } \\ \text { 5c } & \text { Guadalajara } & \text { I6 } 77 & \text { 21X25/8 } & \text { Med. vert. laid. } \\ \text { 5C } & \text { Cordova } & 6 \text { I } 77 & \text { I3 } 1 / 2 \times 1 / 4 & \text { Med. vert. laid. }\end{array}$




Names of Postal Districts surcharged twice.

\begin{tabular}{|c|c|c|c|}
\hline Value. & District. & & Meas. of Surcharge \\
\hline & Mexico & I & \\
\hline IOOC & Colima & 9 & I $31 / 2 \times 21 / 4$ \\
\hline $25 \mathrm{c}$ & C. Victoria & I 2 & I $7 \mathrm{XI} 1 / 2$ \\
\hline $5 \mathrm{c}$ & C. Victoria & 12 & $17 \times 11 / 2$ \\
\hline $25 \mathrm{c}$ & Jalapa & 20 & $\operatorname{IIXI} 3 / 4$ \\
\hline $25 \mathrm{c}$ & Jalapa & 20 & $\operatorname{IIXI} 3 / 4$ \\
\hline $5 \mathrm{Oc}$ & Morelia & 29 & $15 \times 2$ \\
\hline $25 \mathrm{c}$ & Queretaro & 35 & I $41 / 2 \times 13 / 4$ \\
\hline $5 \mathrm{c}$ & Toluca & 46 & I $3 \times 23 / 4$ \\
\hline $100 \mathrm{C}$ & Ures & 49 & $7 \times 2$ \\
\hline IOOC & Vera Cruz & 50 & I $8 \times 13 / 4$ \\
\hline $50 \mathrm{c}$ & Zacatecas & $5 \mathrm{I}$ & I $61 / 2 \times 11 / 2$ \\
\hline $25 \mathrm{c}$ & Tuxpan & 58 & IIXI $5 / 8$ \\
\hline $25 \mathrm{c}$ & Cordova & $6 I$ & I $31 / 3 \times 13 / 4$ \\
\hline
\end{tabular}

Ordinary vertical laid.

Ordinary medium wove.

Thin crisp wove.

Ordinary laid.

Ordinary medium wove.

Ordinary wove

Ordinary medium wove.

Thin crisp wove.

Ordinary medium ". wove.

Wove, watermarked Papel Sellado. Medium laid.

List of stamps known so far on "Papel Sellado" watermark.

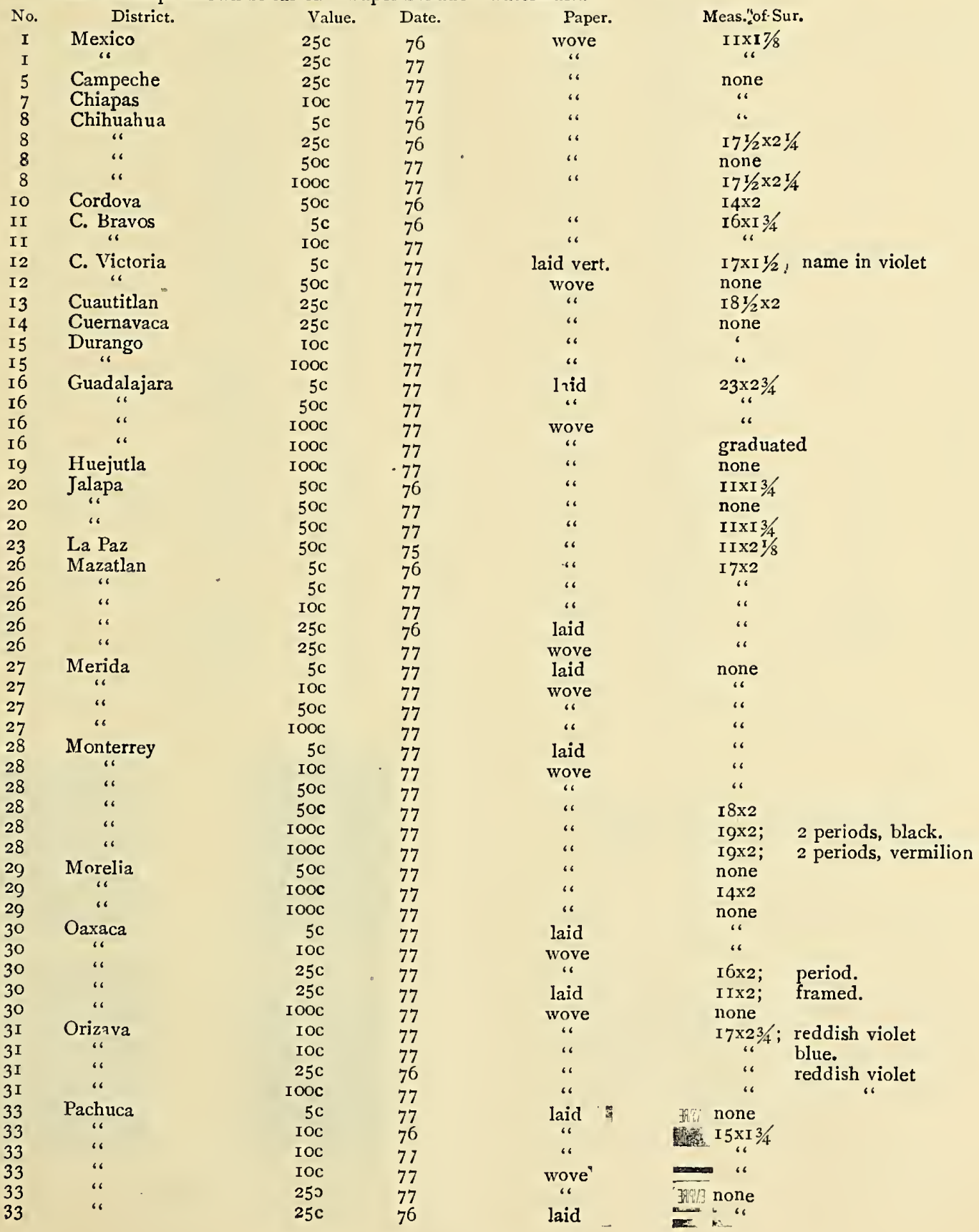




\begin{tabular}{|c|c|c|c|c|c|c|}
\hline No. & District. & Value. & Date. & Paper. & Meas. of sur. & \\
\hline 33 & Pachuca & $25 \mathrm{c}$ & 77 & laid & I5XI $3 / 4$ & \\
\hline 33 & " & IOOC & 76 & wove & “ & \\
\hline 34 & Puebla & $5 \mathrm{c}$ & 77 & laid & $13 \times 2$ & \\
\hline 34 & “" & IOC & 77 & wove & “" & \\
\hline 34 & “" & $25 \mathrm{c}$ & 76 & laid & $13 \times 2$ & \\
\hline 34 & " & $25 \mathrm{c}$ & 76 & wove & "“ & \\
\hline 34 & “" & IOOC & 77 & "، & “" & \\
\hline 34 & “" & IOOC & 77 & “" & none & \\
\hline 35 & Queretaro & IOC & 77 & "“ & $" ،$ & \\
\hline 35 & " & IOOC & 77 & “" & I $41 / 2 \times I^{3} / 4$ & \\
\hline 35 & “. & IOOC & 77 & “" & none & \\
\hline 36 & Saltillo & $5 \mathrm{c}$ & 77 & laid & $" 1$ & - \\
\hline 36 & “ & $5 \mathrm{c}$ & 77 & $"$ " & $141 / 2 \times 17 / 8$ & \\
\hline 36 & “" & IOC & 77 & wove & none & \\
\hline 36 & ، & IOC & 77 & " & $141 / 2 \times 17 / 8$ & \\
\hline 37 & S. L. Potosi & IOC & 77 & laid & none & \\
\hline 37 & “ & $25 \mathrm{c}$ & 77 & $"$ " & $15 \times 13 / 4$ & \\
\hline 37 & “" & $25 \mathrm{c}$ & 77 & wove & "“ & \\
\hline 49 & Ures & $25 \mathrm{c}$ & 77 & laid & $7 \times 2$ & \\
\hline 49 & “ & $25 \mathrm{c}$ & 76 & .. & none & \\
\hline 49 & " & $50 \mathrm{C}$ & 76 & wove & $7 \times 2$ & \\
\hline 49 & “، & $5 \mathrm{Oc}$ & 75 & “ & “" & \\
\hline 49 & "، & $5 \mathrm{Oc}$ & 77 & “" & none & \\
\hline 49 & “" & IOOC & 77 & ؛ & $7 \times 2$ & \\
\hline 50 & Vera Cruz & $5 \mathrm{c}$ & 77 & “" & none & \\
\hline 50 & ". & $5 \mathrm{c}$ & 77 & laid & $18 \times 13 / 4$ & \\
\hline 50 & “" & $5 \mathrm{c}$ & 77 & " & none & \\
\hline 50 & “" & IOC & 77 & wove & I $8 \times x_{1} 3 / 4$ & \\
\hline 50 & “" & $25 \mathrm{c}$ & 77 & "“ & 6 & \\
\hline $5 \mathrm{I}$ & Zacatecas & $5 \mathrm{c}$ & 77 & laid & $16 \times 17 / 8$ & \\
\hline $5 \mathrm{I}$ & " & $5 \mathrm{c}$ & 77 & "“ & none & \\
\hline $5 \mathrm{I}$ & “" & $5 \mathrm{c}$ & 77 & “ & $16 \times 17 / 8$ & \\
\hline $5 \mathrm{I}$ & ، & $50 \mathrm{c}$ & 76 & wove & 16 & \\
\hline $5 \mathrm{I}$ & “ & IOOC & 77 & “" & " & \\
\hline 52 & Guanajuato & $25 \mathrm{c}$ & 77 & ، & none & \\
\hline 52 & $" \%$ & $50 \mathrm{c}$ & 77 & “ & $181 / 2 \times 13 / 4$ & \\
\hline 52 & “ & IOOC & 77 & “" & none & \\
\hline 53 & Cocula & $25 \mathrm{c}$ & 77 & " & IO $1 / 2 \times 11 / 2$ & \\
\hline 53 & "“ & $25 \mathrm{c}$ & 77 & “" & none & \\
\hline 53 & “" & $25 \mathrm{c}$ & 76 & "، & $" 1$ & \\
\hline 53 & “" & $25 \mathrm{c}$ & 77 & "“ & IO $1 / 2 \times 11 / 2$ & \\
\hline 55 & Tepic & $5 \mathrm{Oc}$ & 76 & laid & $81 / 2 \times 11 / 2$ & \\
\hline 56 & Parral & $5 \mathrm{c}$ & 77 & " & none & \\
\hline 56 & " & $5 \mathrm{c}$ & 77 & “" & I $21 / 2 \times$ X $3 / 4$ & \\
\hline 56 & “" & IOC & 77 & wove & ، & \\
\hline 56 & “" & $50 \mathrm{C}$ & 76 & "، & “" & \\
\hline 58 & Tuxpan & $25 \mathrm{c}$ & 76 & “" & $\operatorname{IIXI} 5 / 8$ & \\
\hline 59 & Tula de $T$ & $25 \mathrm{c}$ & 77 & “ & I $41 / 2 \times 13 / 4$ & \\
\hline $6 \mathrm{I}$ & Cordova & $5 \mathrm{c}$ & 77 & laid & $131 / 3 \times 13 / 4$ & \\
\hline $6 I$ & " & IOC & 77 & wove & none & \\
\hline 61 & “ & IOC & 77 & “" & $131 / 2 \times 13 / 4$ & \\
\hline
\end{tabular}

I878-83.

Head of Hidalgo to left. Same designs as issue of 1874 but surcharged with number

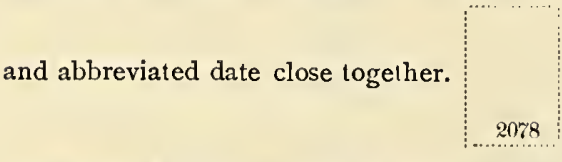

During the first few months of 1878 the number and date were printed uniformly at the bottom of the stamp, but laler all (with the exception of those used in the district of Mexico) had the number and dale printed in the upper half of the stamp, usually in the right hand upper quarter.
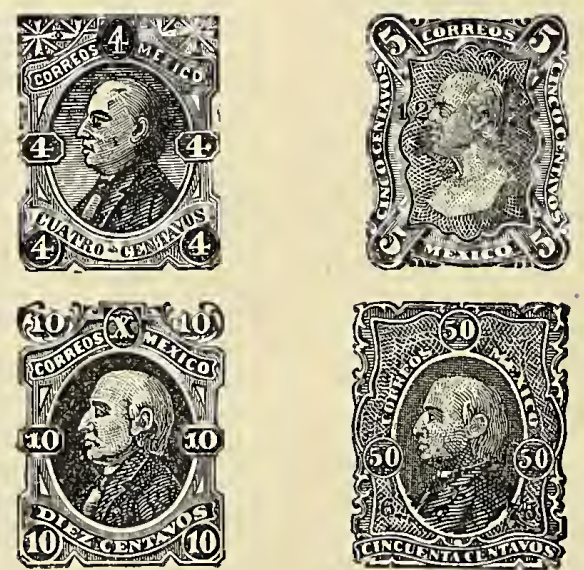

Printed on various papers. Perforated I2, 
. 

I. Surcharged with number and abbreviated date only.

$I^{\circ}$ On rather thin soft wove paper. (I878 and $188 \mathrm{r}-82$.)

\begin{tabular}{|c|c|c|}
\hline $\begin{array}{l}\text { I } 56 \text { I } \\
\text { I } 562\end{array}$ & $\begin{array}{l}\text { 4c dull brownish red } \\
5 \mathrm{c} \text { chestnut brown }\end{array}$ & I88I -82 \\
\hline $\begin{array}{l}1563 \\
1563\end{array}$ & $5 \mathrm{c}$ dull brown & “. \\
\hline I 564 & IOc black & $18 ; 8$ \\
\hline 56 & IOc mustard yellow & \\
\hline 56 & Ioc reddish orange & $\mathrm{r} 88 \mathrm{I}-82$ \\
\hline 56 & $25 \mathrm{c}$ dark steel blue & 1878 \\
\hline I 56 & $25 \mathrm{c}$ bright blue (shades) & I $88 \mathrm{I}-82$ \\
\hline I 569 & $50 c$ green? & \\
\hline$=$ & Doc carmine? & \\
\hline
\end{tabular}

1570 IOOC carmine?

$2^{\circ}$ On rather thin soft paper, laid ver. tically in narrow lines. (I88I-82.)

I57I $4 \mathrm{C}$ dull brownish red

I $572 \quad 5 \mathrm{c}$ chestnut brown

I 573 Ioc reddish orange

$157425 \mathrm{c}$ bright blue (shades)

I575 50c dark bluish green

I576 IOOc carmine

Variety: Unperforated.

I $577 \quad 25 \mathrm{c}$ bright blue

The only specimens known are surcharged 378I (Guadalajara)

$3^{\circ}$ On rather thin paper, laid horizontally with broad heavy lines. (1878-79.)

I578 $5 \mathrm{C}$ chestnut brown

$4^{\circ}$ On ordinary wove paper, varying from from medium to thick. (I878-83.)

\begin{tabular}{|c|c|c|}
\hline I579 & $4 \mathrm{c}$ dull brownish red & $\dot{\mathbf{I}} 88 \mathrm{o}-83$ \\
\hline I 580 & $5 \mathrm{c}$ chestnut brown & $1878-82$ \\
\hline I58I & $5 \mathrm{c}$ brown & $1880-82$ \\
\hline I582 & $5 \mathrm{c}$ light brown & \\
\hline 1583 & IOc black & 1878 \\
\hline I584 & IOc mustard yellow & \\
\hline I585 & roc orange yellow & $1878-82$ \\
\hline I 586 & Ioc reddish orange & I $88 \mathrm{I}-82$ \\
\hline I 587 & 25c dark steel blue & I $878-83$ \\
\hline I588 & $25 \mathrm{c}$ dark greenish blue & $1878-79$ \\
\hline I 589 & $25 \mathrm{c}$ bright Prussian blue & I879 \\
\hline I 590 & $25 \mathrm{c}$ blue & I878-8I \\
\hline I $59 \mathrm{I}$ & $25 \mathrm{c}$ dark blue & \\
\hline 1592 & $25 \mathrm{c}$ light blue & 1879 \\
\hline I593 & $50 \mathrm{c}$ yellowish green & $\mathbf{1} 878$ \\
\hline I594 & $50 c$ pale green & \\
\hline I595 & $50 c$ dark green & I878-8I \\
\hline I 596 & $50 \mathrm{cgreen}$ & \\
\hline I 597 & Iooc dull dark carmine & I 878-79 \\
\hline I598 & Iooc dull pale carmine & I879-80 \\
\hline I59 & IOOc carmine & \\
\hline
\end{tabular}

I 599 IOOC carmine

$5^{\circ}$ On ordinary paper, varying from medium to thick, laid vertically with narrow lines. (1878-83.)

I600 $4 \mathrm{c}$ dull brownish red (shades)

I60I $5 \mathrm{c}$ brown I880-83

I603 5c chestnut brown I878-82

I604 Ioc orange yellow "“

I605 Ioc pale orange yellow ".

I607 25c dark greenish blue I878-79

I608 25c dark blue I879-8I

r6og $25 \mathrm{c}$ blue

I6Io $25 \mathrm{c}$ light blue

I6I I 5Oc green

I6I2 50c dark green

I6I3 IOOc carmine

I6I4 Iooc dark carmine

$6^{\circ}$ On ordinary paper laid horizontally with narrow lines. (I880-8I.)

$16 \pi 5$ Inc orange yellow (shades) $7^{\circ}$ On wove paper, varying slightly in thickness, having quadrille appearance and watermarked PAPEL SELLADO in sheet. (I880-8I.)

I6I6 Iooc pale carmine

$8^{\circ}$ On rather thick vertically laid paper, watermarked PAPEL SELLADO in sheet. (I880.)

I6I $75 \mathrm{c}$ brown

I6I8 Iooc dull carmine

I6I9 Iooc dull pale red

$9^{\circ}$ On very thin wove paper. (I88I-83.)

I620 $4 \mathrm{c}$ dull brownish red (shades)

I62I $5 \mathrm{c}$ brown

$1622 \quad 5 \mathrm{c}$ dull brown

I623 5c dark brown

I624 5c brownish red

I625 IOc yellow

I626 Ioc orange yellow

1627 IOc reddish orange

I $62825 \mathrm{c}$ blue

I629 25c pale blue

I630 $25 \mathrm{c}$ dark blue

I63I 25c Prussian blue

I632 50c green

I633 5oc bluish green

I634 Iooc carmine

I635 Iooc dark carmine

II. Surcharged with number and abbreviated date, and also with name of postal district.

$I^{\circ}$ On rather thin soft wove paper. (I 878 and $188 \mathrm{I}-82$.)

I636 4c dull brownish red I $88 \mathrm{I} \cdot 8$

$16375 \mathrm{c}$ chestnut brown "“

I $638 \quad 5 \mathrm{c}$ dull brown

I639 Ioc black

r640 roc mustard yellow

I64I Ioc reddish orange

I642 $25 \mathrm{c}$ dark steel blue $\quad$ I 878

I643 25c bright blue (shades) I88I-82

I644 50c green ?

I645 Iooc carmine?

$2^{\circ}$ On rather thin soft paper, laid vertically with narrow lines. (I88 I-82.)

I646 4c dull brownish red

I647 5c chestnut brown

1648 Ioc red dish orange

I649 25c bright blue (shades)

I650 5oc dark bluish green

I65I Iooc carmine

$3^{\circ}$ On rather thin paper, laid horizontally with broad heavy lines. (I 878-79.)

I652 5c chestnut brown I653 Ioc black

This last, on this paper, with number and date close together is an excessively rare stamp; we have seen but one which is in Mr. Lawrence's collection, and which is surcharged Morelia I5×2, 2478.

$4^{\circ}$ Ordinary wove paper, varying from medium to thick. (I878-83,)

I654 4c dull brownish red I880-83

I655 5c chestnut brown I878-82

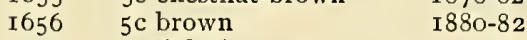

I657 5c light brown

I658 IOc black

I659 Ioc mustard yellow

Ioc orange "

IOc reddish orange

I662 25c dark steel blue $1878-83$

$166325 \mathrm{c}$ dark greenish blue $\quad$ I878-79

I664 $25 \mathrm{c}$ bright Prussian blue I879

I $66525 \mathrm{c}$ blue I878-8

I666 25c dark blue

r667 25c light blue 


\begin{tabular}{|c|c|c|}
\hline 668 & $50 c$ yellowish green & 1878 \\
\hline I669 & $50 c$ pale green & \\
\hline 167 & $50 c$ dark green & 78 \\
\hline 1671 & $50 \mathrm{c}$ green & \\
\hline 1672 & Iooc dull dark carmine & $1878-79$ \\
\hline 1673 & Iooc dull pale carmine & $1879-80$ \\
\hline 167 & IOOc carmine & 1878.82 \\
\hline
\end{tabular}

$5^{\circ}$ On ordinary paper, varying from medium to thick, laid vertically with narrow lines. (1878-83.)

I675 4c dull brownish red (shades)

I676 5c brown I880-83

I678 5c chestnut brown I872-82

I679 Ioc orange yellow

I680 Ioc pale orange yellow

I68I IOc dark orange yellow

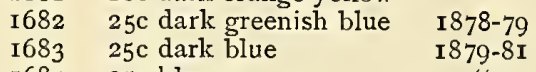

I684 $25 \mathrm{c}$ blue

I685 $25 \mathrm{c}$ bright blue

I686 50c green

I687 50c dark green

I688 Iooc carmine

I689 Iooc dark carmine

$6^{\circ}$ On ordinary paper, laid horizontally

with narrow lines, (I878 and I880-8I.)

I69o Ioc orange yellow (shades)

$7^{\circ}$ On wove paper, varying slightly in

thickness, having quadrille appearance, and watermarked PAPEL SELLADO in sheet. (I878 and I880-8I.)

I69I $25 \mathrm{c}$ dull pale blue $\quad \mathbf{1 8 7 8}$

1692 Iooc pale carmine $\quad$ I880-8I

$8^{\circ}$ On rather thick, vertically laid paper. watermarked PAPEL SELLADO in sheet. (I880.)

I693 Iooc pale carmine

I694 Iooc dull pale red

$9^{\circ}$ On very thick, wove paper. (I88I-83.)

I695 4c dull brownish red (shades)

I696 5c brown

I697 5c pale brown

I698 5c dark brown

$16995 \mathrm{c}$ reddish brown

I700 IOc yellow

I 701 IOc orange yellow

1702 IOc reddish orange

I $70325 \mathrm{c}$ blue

I $70425 \mathrm{c}$ pale blue

I $70525 \mathrm{c}$ dark blue

I706 25c Prussian blue

I707 50c green

I $70850 c$ bluish green

I 709 IOOC carmine

I7I0 rooc dull carmine

III. Surcharged with number and date only in a larger type than that formerly used; the figure " 2 " has a curved tail instead of straight; the figures " 5 " and " 7 " have curved tops instead of straight. The other figures show slight differences, but all are larger than the type last used.

$I^{\circ}$ On ordinary paper, laid vertically with narrow lines. (I883.)

I7II 4c dull brownish red

$2^{0}$ Very thin, wove paper.

(I883.)

I 7I $24 \mathrm{c}$ dull brownish red

I7I3 $5 \mathrm{c}$ brown

I7 I4 IOc orange yellow

I7I5 $25 \mathrm{c}$ blue

I 7 I6 $25 \mathrm{c}$ pale blue

I 7 I $725 \mathrm{c}$ dark blue
I7I8 $50 \mathrm{c}$ bluish green

I719 IOOc bright pinkish carmine

$3^{\circ}$ Very thin paper vertically laid with broad lines.

I720 $4 \mathrm{c}$ dull brownish red

I 72I IOc orange

IV. Same as last, with number and date and also name of postal district.

On ordinary wove paper, laid vertically with narrow lines. (I883.)

I722 4c dull brownish red

I723 $5 \mathrm{C}$ brown

I724 Ioc orange yellow

I725 25c blue

I $72625 \mathrm{c}$ pale blue

I $727 \quad 25 \mathrm{c}$ dark blue

I728 $50 \mathrm{c}$ bluish green

I 729 IOoc bright pinkish carmine

V. Surcharged with name of postal district only.

$I^{\circ}$ On ordinary paper, laid vertically with narrow lines.

I730 $4 \mathrm{c}$ dull brownish red

$2^{\circ}$ Ordinary wove paper.

I 73I $25 \mathrm{C}$ blue

$3^{\circ}$ Thin, soft paper, laid vertically with narrow lines.

$1732 \quad 25 \mathrm{c}$ blue

$4^{\circ}$ Very thin, wove paper.

I $7334 \mathrm{c}$ dull brownish red

I734 $5 \mathrm{c}$ brown

I735 IOc orange yellow

I 736 Ioc reddish orange

$173725 \mathrm{c}$ pale blue

I738 $25 \mathrm{c}$ blue

I739 25c dark blue

I 740 50c green

I74I Iooc carmine (Heitman's Hand Buch)

$5^{\circ}$ Very thin paper, vertically laid with broad lines.

I742 4c dull brownish red

I743 Ioc orange

VI. Without any surcharge, (1884,)

$\mathbf{I}^{\circ}$ On ordinary paper, laid vertically with narrow lines.

I 744. 4c dull brownish red

I 745 IOc orange yellow

$2^{\circ}$ Ordinary wove paper.

I746 4c dull brownish red

$3^{\circ}$ On very thin wove paper.

I747 4c dull brownish red

I748 5c pale brown

I749 IOc reddish orange

I $750 \quad 25 \mathrm{c}$ dark blue

I75I 5 Oc green

I752 rooc carmine (Heitman's Hand Buch)

$4^{\circ}$ Very thin paper, vertically laid with broad lines.

I $753 \quad 4 \mathrm{c}$ dull brownish red

I754 Ioc orange

Variety: Unperforated.

I 755 IOc orange

We have seen used specimens of all the above with the exception of the IOOC carmine.

Stamps of Mexico and Vera Cruz are found with number and date printed in different types from anything heretofore described and having the appearance of being hand stamped instead of printed; we have seen:

$$
\begin{array}{llll}
\text { Mexico IIXI } 7 / 8 & 25 \mathrm{C} & 5483
\end{array}
$$


• 



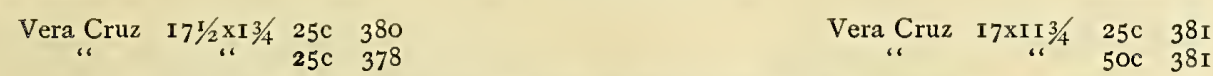

Names of Postal Districts found surcharged on stamps of this Issue. 20. Numbers running from $\mathrm{I}$ to 55 .

All the numbers can be found surcharged with number and date only, except perhaps Mexico.

\begin{tabular}{|c|c|c|}
\hline 1878 & 1 ZAMORA I & I $3 \times 1 \mathrm{I} / 2 ;$ blue \\
\hline I 879 & “ & I $3 \times$ I $1 / 2$; bluish violet \\
\hline I $879-81$ & “ & I $3 \times 1 \mathrm{I} / 2 ;$ dark violet \\
\hline I $88 \mathrm{I}-83$ & “ & I $3 \times 1 \mathrm{I} / 2 ;$ black \\
\hline I $878-8 I$ & 2 ZACATECAS & $161 / 2 \times 2$; black \\
\hline $1878-81$ & ") & I6xI7/8; black \\
\hline $379-8 I$ & “ & I6xI $7 / 8$; blue \\
\hline 82 & “" & I $61 / 2 \times 2$; vermilion \\
\hline $1882-83$ & “ & I $61 / 2 \times 2 ;$ carmine \\
\hline $82-83$ & “" & I6xI $7 / 8 ;$ carmine \\
\hline $32-83$ & “" & I6 $1 / 2 \times 2$; violet \\
\hline
\end{tabular}

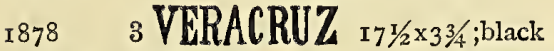

I878-79 VERACRUZ I8x3-I $1 / 2$; black

I878-8I VERACRUZ I3 2 ; black

I878-83 VERACRUZ $171 / 2 \times 13 / 4$; black

I880-8I VERACHUZ I5 XI $1 / 2$; black

I883 VERACRUZ $171 \frac{1}{2} \times 2$ 1/2; black

I878-79 4 URES $7 \times 2$; black

I880 HERMOSILLO $20 \times 3$; violet

1879-83 " 3 20x3; black

1879-83 HERMOSILLO 20x3; black

I878-82 5 TULANCINGO I8x2; black

1878-82 " I8x2; blue

1880 TULANCINCO $18 \times 2-3$; black

1882-83 TULANCINGO $23 \times 3$; black

I878-83 6 TULA DE T. I5 $1 / 4 \times 13 / 4$, with

I878-83 7 TULA $8 \times 13$; ; black

r $880-8 \mathrm{I} \quad$ " $\quad 8 \times 13 / 4$; blue

I $88 \mathrm{I}-82 \quad$ " $8 \times 1$ I $3 /$; carmine

I 878-82 8 TUXPAN IIXI $5 \%$; black

I88I-82 " " IIXI $5 / 8$; blue

I $882-83 \quad$ " $\quad$ IIXI $5 / 8$; carmine

I882 “" IIXI $5 / 8$; brown

I883 " IIXI $5 / 8$; violet

I88I-82 TUSPAN IIX2 $1 / 2$; black

I $88 \mathrm{I}-8 \mathrm{2}$ " $\quad$ IIX2 $\mathrm{I} / 2$; blue

I882 “ IIX $2 \frac{1}{2}$; carmine

I 8789 TOLUCA I3xI $3 / 4$; blue

1879-80 TOLUCA. I3 $1 / 2 \times 21 / 2$, with period; blue

1880-82 TOLUCA $143 / 4 \times 3$; blue

$\mathrm{I} 882 \quad$ " I $43 / 4 \times 3$; violet

I882-83 TOLUCA. I $5 \times 21 / 2$, with period; violet

I 87810 TEHUACAN $15 \%$ I $2 \times 17 / 8$; black

I $878-80 \quad$ " $\quad 151 / 2 \times 17 / 8$; blue

I880-83 “ $\quad$ I $51 / 2 \times 17 / 8$; bright rose

I 883

I883
$15 \mathrm{x} / 2 \times 1 / 8$; carmine

TEHUACAN $15 \% 2 \times 3-17 / 8$; car-
I878-83 11 TEXCOCO I3XI 1 $1 / 2$; black

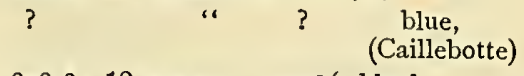

I878-83 12 TEPIC 9xI 1/2; black

$1880 \quad$ " $9 \times 1 \frac{1}{2}$; blue

I882 " $\quad 9 \times 1 \mathrm{I} / 2$; reddish violet

I883 TEPIC $7 \times 2$; black

1883 “ $7 \times 2$; blue

$1883 \quad$ “ $7 \times 2$; reddish violet

? 13 TLAXCALA I5xI $3 / 4$; black

1878 TLAXCALA I $4 \frac{1}{2} \times 13 / 4$; black

I $879-83 \quad$ " I $4 \frac{1}{2} \times 13 / 4$; blue

I878-79 14 - TAMPICO - I61/2x2, between periods; black

1879 ? " I6 $1 / 2 \times 2$, between

I878-80 TAMPICO I5 $1 / 2 \times 2$; black

I880-8 I “ $\quad 15 \mathrm{I} / 2 \times 2$; blue

I88 I “ $\quad 151 / 2 \times 2$; vermilion

I88I “ $\quad \mathrm{I} 5 \mathrm{I} / 2 \mathrm{x} 2$; violet

I88I-82 TAMPICO I4XI $3 / 4$; violet

1882-83 " ${ }^{\text {I4 } 4 \times 13 / 4 \text {; black }}$

I882 TAMPICO $18 \times_{3}$; violet

? “ $18 \times 3$; blue

I882-83 . “ I8x3; black

I878-83 15 TACUBAYA $141 / 2 \times 11 / 2$; blue

I878-83 16 TABASC0 $141 / 2 \times 13 / 4$; black

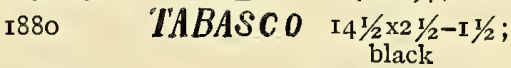

? TABASCO I3xI $1 / 2$; black

I878-80 17 S.L.POTOSI I5XI 3/4; black

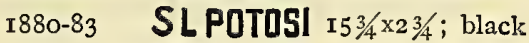

? 18 SALTILLO. I6 $1 / 2 \times 21 / 2$, with

period; black

? SALTILLO I5 $54-2$; black

I878-83 SALTILLO I5 $21 / 2$; black

I 878-83 19 OUERETARO I 4 1 $/ 2 \times 1$ I $3 / 4$; black

I $883 \quad$ " $141 / 2 \times 13 / 4$; blue

$1883 \quad$ " $\quad 141 / 2 \times 13 / 4$; violet

? QUERETARO $\begin{gathered}\text { I } 41 / 2 \times 11 / 2-21 / 8 ; \\ \text { black }\end{gathered}$

? QUERETARO I4 $1 / 2 \times 2-1$; black

I883 OUERETARO $20 \times 3-2$; black

I 87820 PUEBLA . I $4 \times 13 / 4$, with

period; black

I $878-83$ PUEBLA I3×2; black

I882-83 " I3x2; reddish

$1882 \quad$.. I3x2; blue

I878 PUEBLA. I $3 \times 23 / 4$; black

? $21 \mathrm{PACHUCA}$. I6 $2 \mathrm{I} / 4$, with period; black

? " $\quad 16 \times 21 / 4$, with

period; blue

I878-83 PACHUCA I5 5 I $3 / 4$; black 


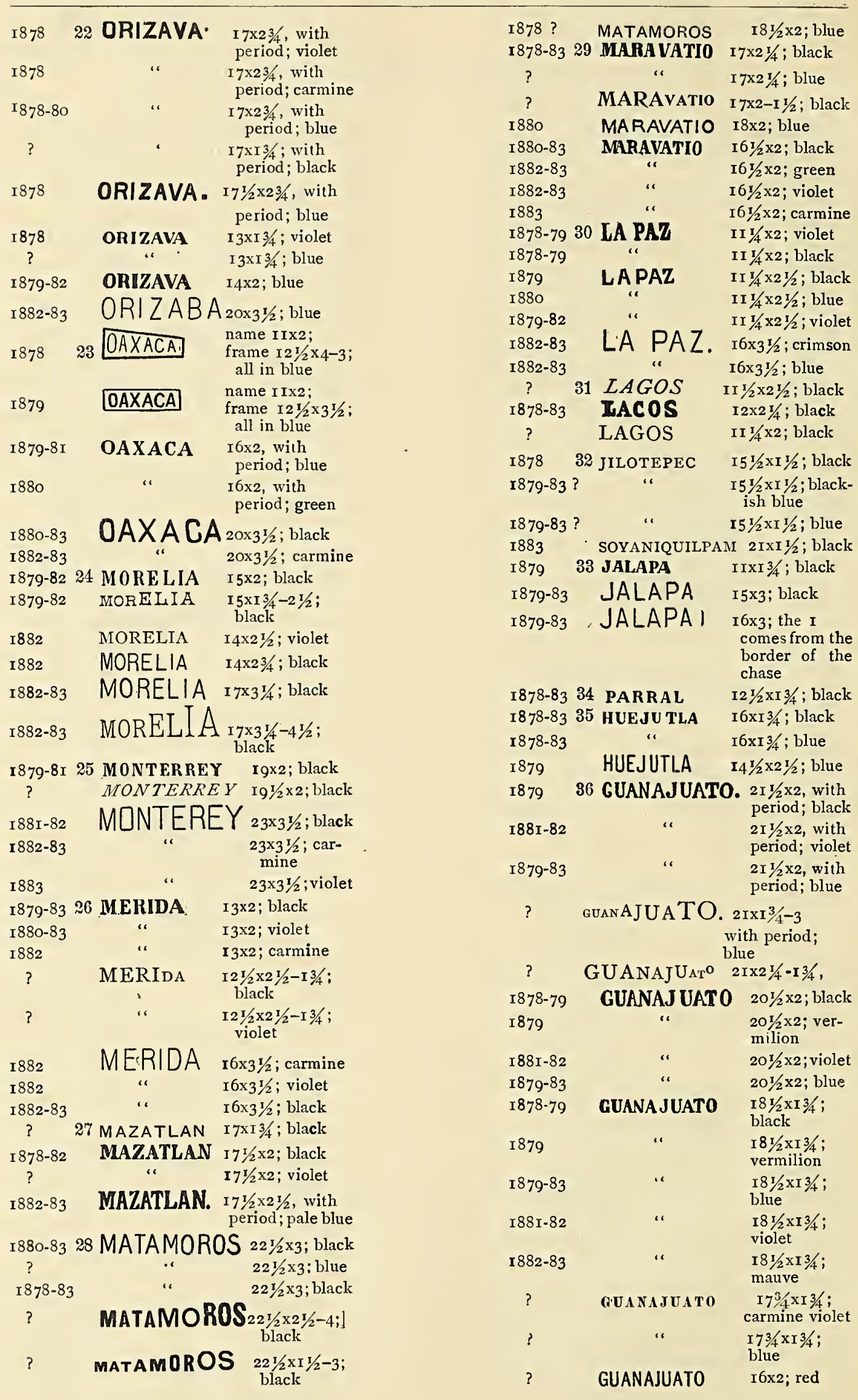






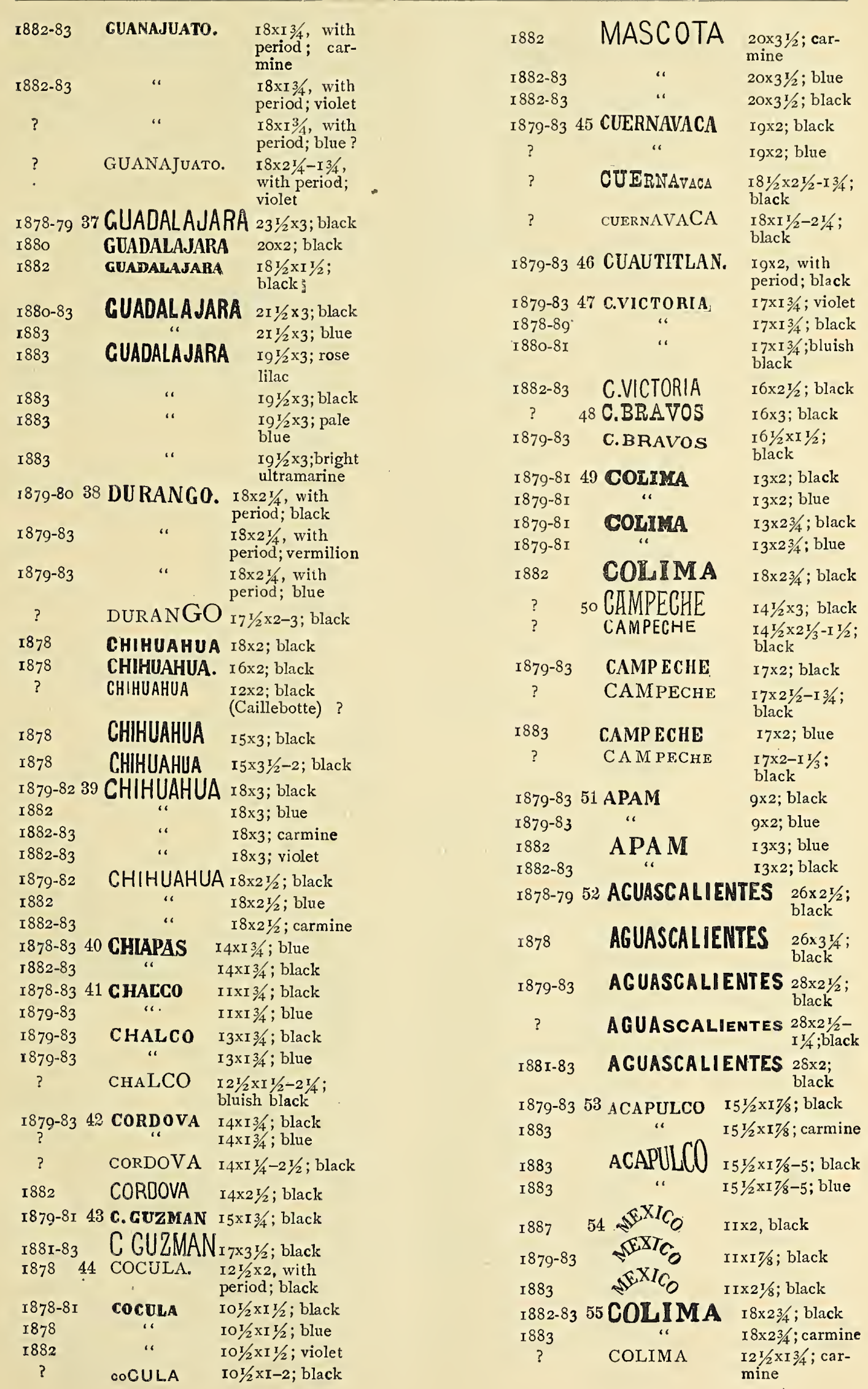


Errors.

Issues of $1878-82$. First type, (head to left).

Names of postal districts found surcharged two or more times on the stamps of these isssue.

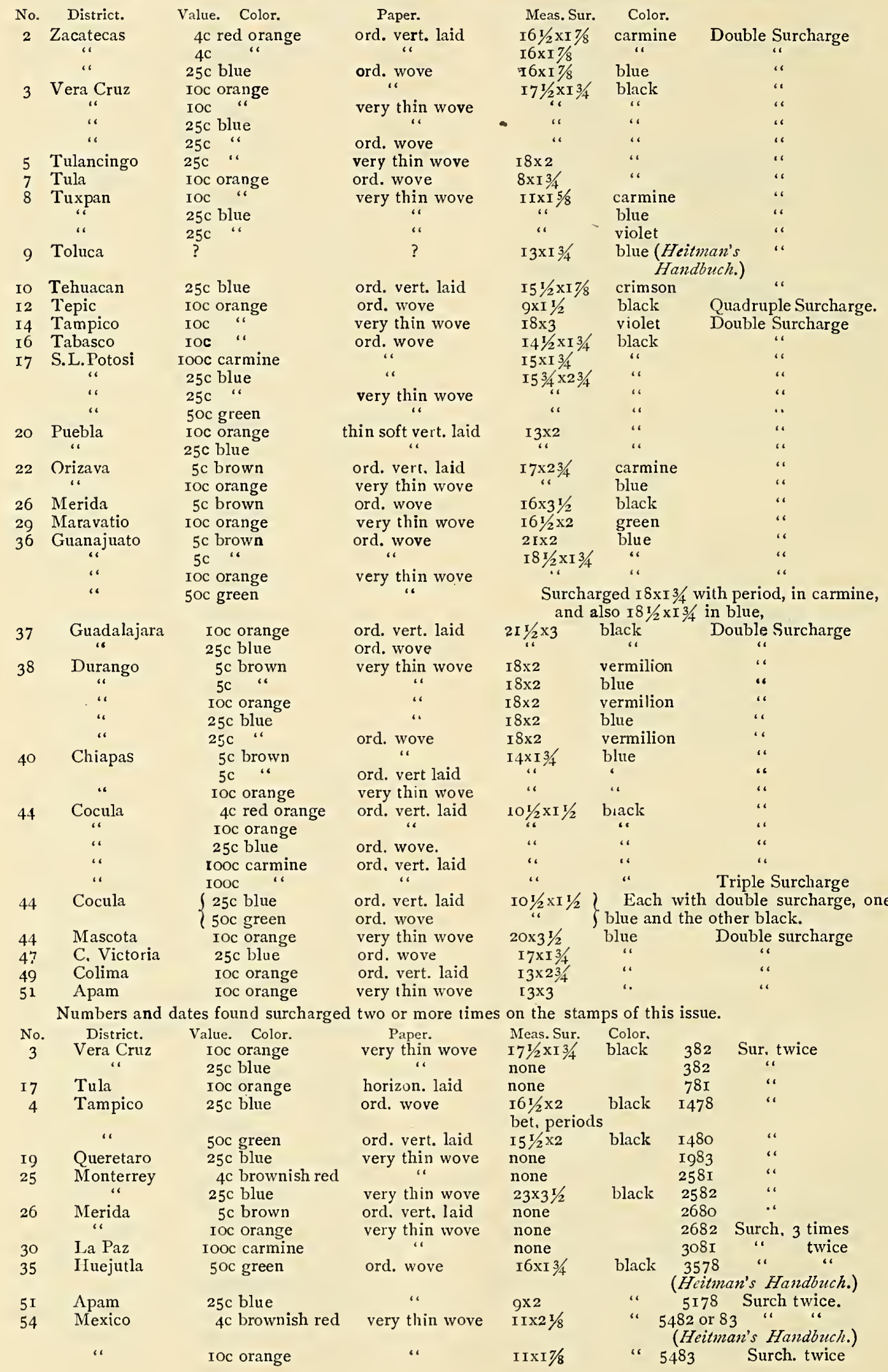




Surcharged with date only.

$\begin{array}{cc}\text { District. } & \text { Value. Color. } \\ \text { Chiapas } & 50 \mathrm{c} \text { green } \\ \text { Cordova } & \text { IOc orange } \\ \text { ? } & \text { IOc " } \\ \text { Tuxpan } & 5 \mathrm{c} \text { brown } \\ ? & \text { IOc orange } \\ \text { Apam } & 25 \mathrm{c} \text { blue }\end{array}$

$\begin{array}{ccl}\text { Paper. } & \text { Meas. Sur. } & \text { Color. } \\ \text { very thin wove } & \text { I } 4 \times 13 / 4 & \text { blue } \\ \text { "“ } & \text { I4xI3/4 } & \text { black } \\ \text { none } & \\ \text { ord. vert. laid. } & \text { IIxI5/8 } & \text { black } \\ \text { ord. wove } & \text { none } & \\ \text { ord. vert, laid. } & 9 \times 2 & \text { black }\end{array}$

Surcharged with number only.

District. Value. Color.

Paper. Meas. Surch. Color.

ord, vert laid $\quad 171 / 2 \times 23 / 4$ blue

No. 22.

The following are known to us surcharged with name of postal district only :

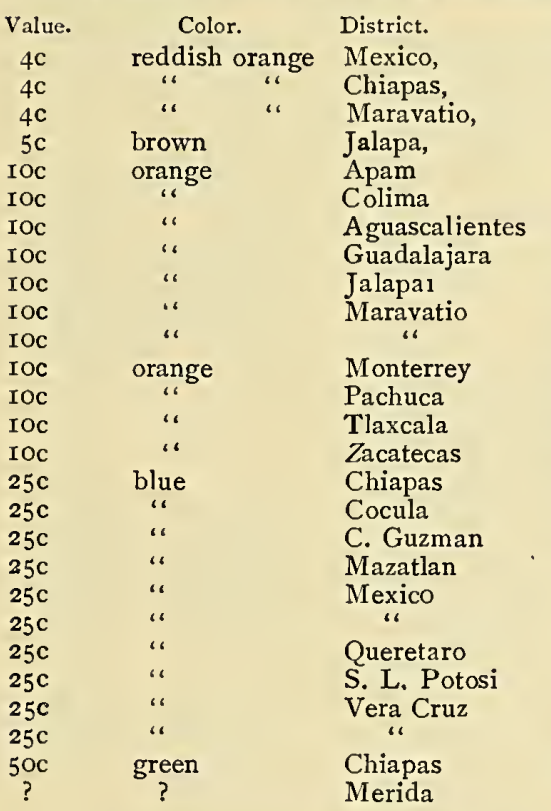

\begin{tabular}{|c|c|}
\hline Color. & Meas. Surch. \\
\hline black & $\operatorname{IIXI} 7 / 8$ \\
\hline “ & $14 \times 13 / 4$ \\
\hline “ & $161 / 2 \times 2$ \\
\hline “، & I $5 \times 3$ \\
\hline " & $13 \times 3$ \\
\hline “ & $18 \times 23 / 4$ \\
\hline " & $28 \times 2$ \\
\hline pale blue & $191 / 2 \times 3$ \\
\hline black & $16 \times 3$ \\
\hline violet & $161 / 2 \times 2$ \\
\hline black & $16 \frac{1}{2} \times 2$ \\
\hline violet & $23 \times 31 / 2$ \\
\hline black & I $5 \times 13 / 4$ \\
\hline blue & I $41 / 2 \times 13 / 4$ \\
\hline carmine & $16 \times 17 / 8$ \\
\hline blue & I 4 XI $3 / 4$ \\
\hline " & $101 / 2 \times 1 / 2$ \\
\hline black & $17 \times 31 / 2$ \\
\hline ") & $171 / 2 \times 2$ \\
\hline “ & $\operatorname{IIXI} 7 / 8$ \\
\hline " & " \\
\hline "6 & $141 / 2 \times 13 / 4$ \\
\hline " & I $53 / 4 \times 23 / 4$ \\
\hline ") & $171 / 2 \times 13 / 4$ \\
\hline " & \\
\hline $\begin{array}{c}\text { black } \\
\text { ? }\end{array}$ & $\begin{array}{c}14 \times 13 / 4 \\
?\end{array}$ \\
\hline
\end{tabular}

Paper. ordinary vert. laid very thin wove is

“

very thin wove c

ordinary wove

$$
16
$$

thin soft vert. laid

ord. vert laid

thin soft vert. laid

ordinary wove

very thin wove

?

(Heitman's Handbuch.) carmine.

Varieties known to us surcharged on paper watermarked "Papel Sellado" in sheet, all roo centavos

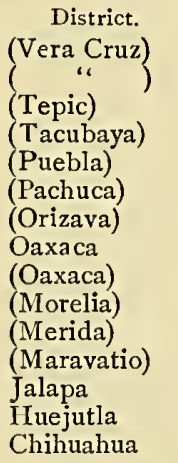

No.and Date.
380
380
1280
1580
2080
2180
2281
2380
2380
2480
2680
2980
3380
3580
3980

Meas. of Sur.
none
none
none
none
nore
none
none
I6x2
none
“.
“"
$15 \times 3$
$16 \times 134$
$18 \times 3$

Color.
dark blue
black
blue
black

Paper.
wove
vert. laid
wove
“"
“
“"
vert. laid
wove
“"
“.
“"

Errors in surcharge of Numbers on issues 1878-82, first type.

\begin{tabular}{|c|c|c|c|c|c|c|}
\hline $\begin{array}{c}\text { District. } \\
\text { Zacatecas }\end{array}$ & $\begin{array}{l}\text { Value. Color. } \\
\text { roc orange } \\
25 \mathrm{c} \text { blue }\end{array}$ & $\begin{array}{l}883 \\
883\end{array}$ & $\begin{array}{l}\text { stead of } \\
\text { “" }\end{array}$ & $\begin{array}{l}283 \\
283\end{array}$ & $\begin{array}{l}\text { Paper. } \\
\text { very thin wove }\end{array}$ & $\begin{array}{l}\text { Meas. Sur. Color. } \\
16 \times 17 / 8 \text { carmine } \\
\text { “ }\end{array}$ \\
\hline “" & $25 \mathrm{c} "$ & 883 & “ & 283 & “ & (strip of 3 ) \\
\hline Vera Cruz & $4 c$ red orange & I38I & “" & $38 \mathrm{I}$ & ord. vert. laid & “ \\
\hline "، & Ioc orange & 883 & “ & 383 & “ & I $71 / 2 \times 13 / 4$ black \\
\hline “" & $25 \mathrm{c}$ blue & 883 & “" & 383 & “ & “" \\
\hline Tulancingo & $25 \mathrm{c}$ “" & $28 I$ & “" & $38 \mathrm{I}$ & thin soft vert. laid & I $5 \times 1 / 2$ \\
\hline Tulancingo & $\begin{array}{l}5 \mathrm{c} \text { brown } \\
25 \mathrm{c} \text { blue }\end{array}$ & $\begin{array}{l}58 \mathrm{I} \\
58 \mathrm{I}\end{array}$ & “، & $\begin{array}{l}68 \mathrm{I} \\
68 \mathrm{I}\end{array}$ & ord. "vert. laid & $18 \times 2$ \\
\hline Tula & $25 \mathrm{c}$ & 27? & “ & 7 ? & ? & (Heitman's Handbuch) \\
\hline
\end{tabular}




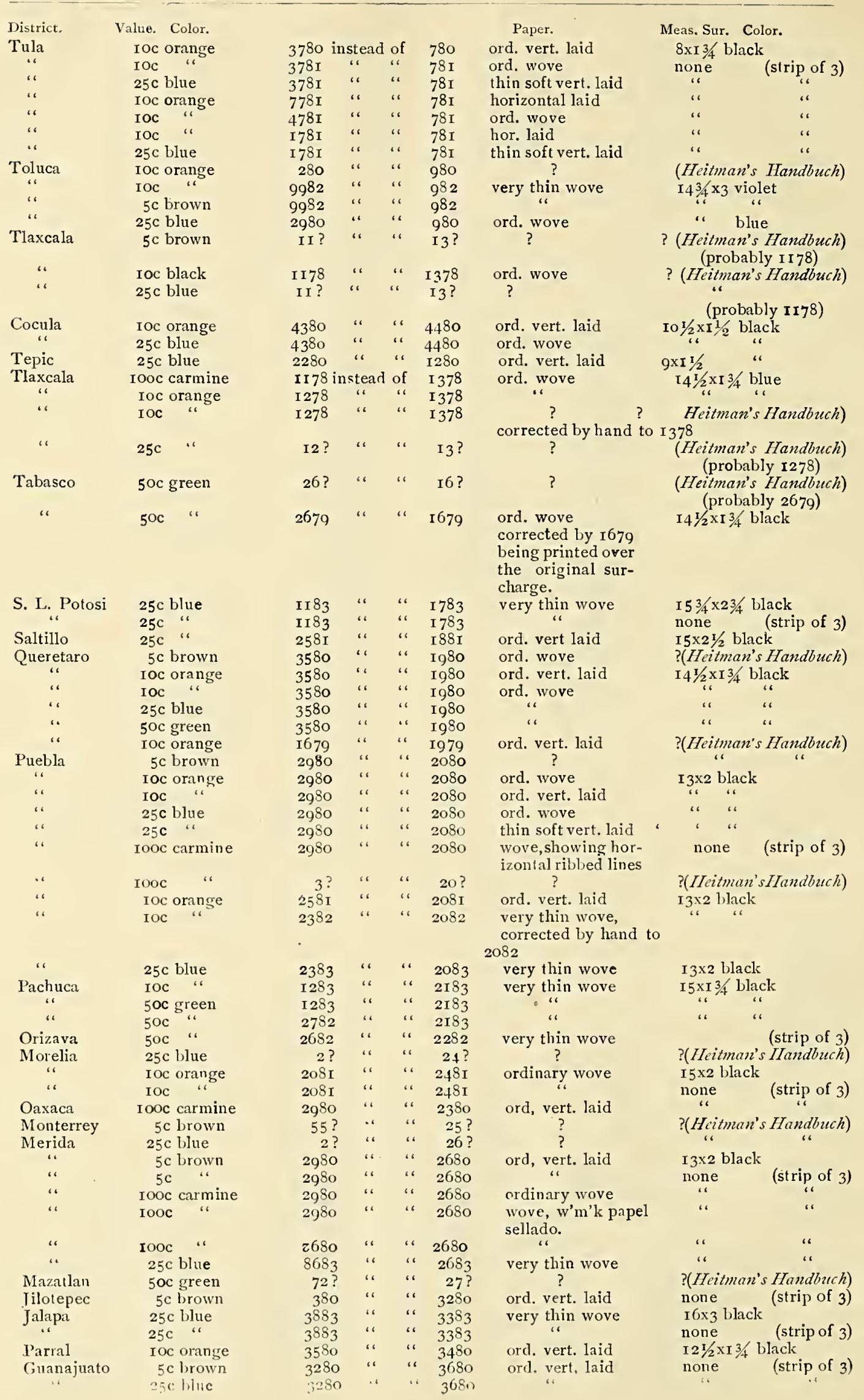




$$
\text { . }
$$





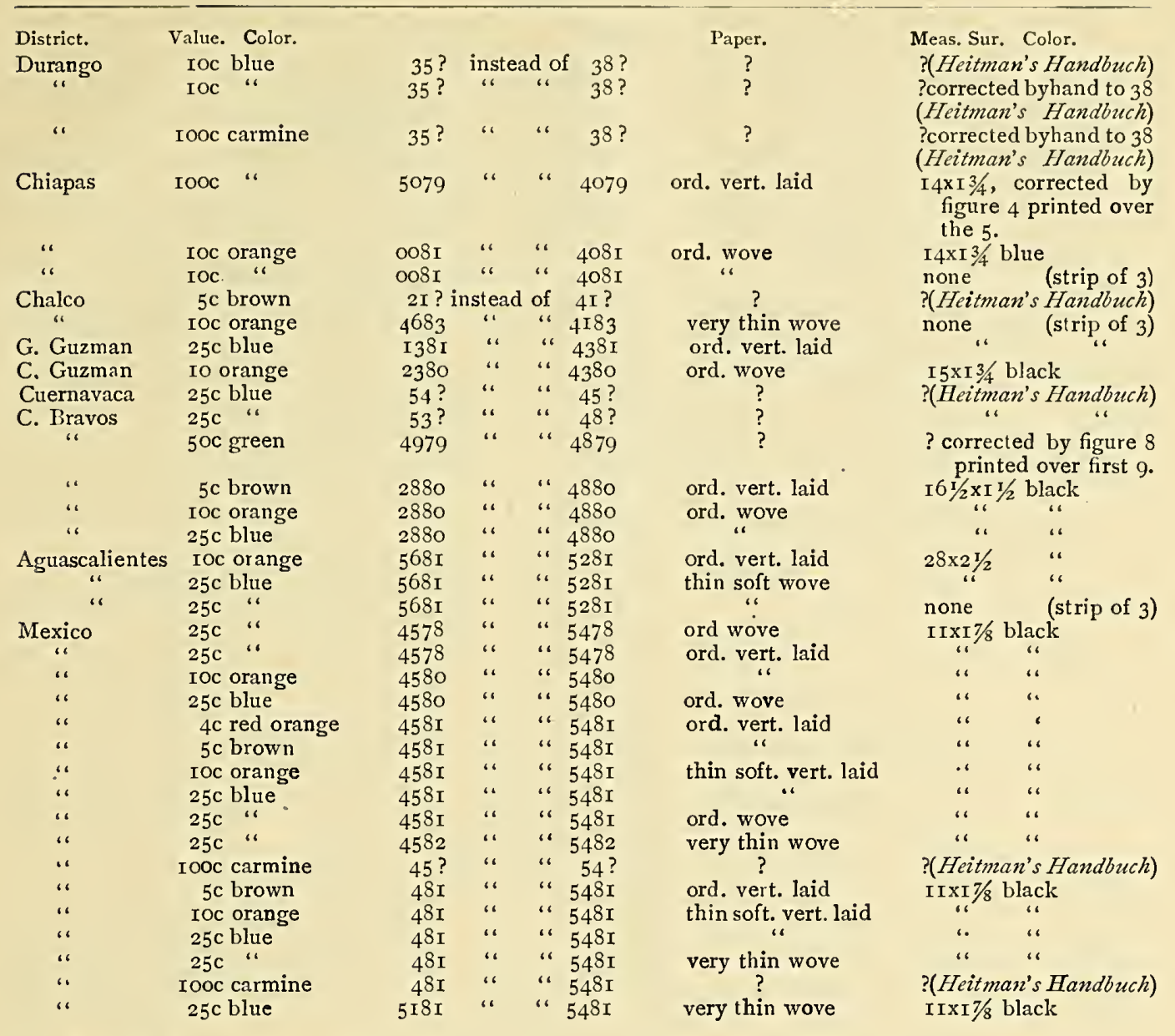

Errors in surcharge of dates.

District. Value. Color.

\begin{tabular}{|c|c|c|c|c|}
\hline Zamora & $50 c$ green & \multicolumn{2}{|c|}{ I 28 instead of } & I 82 \\
\hline Vera Cruz & $25 \mathrm{c}$ blue & 328 & $" 1$ & 382 \\
\hline Hermosillo & $25 \mathrm{c}$ & 480 & 66 & $48 I$ \\
\hline Tacubaya & 5 oc green & $80 I_{5}$ & ‘. & I 580 \\
\hline $\mathrm{La} \mathrm{Paz}$ & IOC orange & $30 \mathrm{I}$ & “ & $308 I$ \\
\hline Jalapa & IOC & 3333 & “ & $338 \mathrm{I}$ \\
\hline " & 4c br'wn rerl & $339 I$ & " & $338 I$ \\
\hline ‘. & $25 \mathrm{c}$ blue & 3391 & 66 & $338 I$ \\
\hline Durango & $5 \mathrm{c}$ brown & 3868 & " & $388 \mathrm{I}$ \\
\hline "1 & IOc orange & 3868 & "، & $388 I$ \\
\hline “" & $25 \mathrm{c}$ blue & 3868 & “ & $388 \mathbf{I}$ \\
\hline “، & $5 \mathrm{c}$ brown & 3880 & “ & $388 \mathrm{I}$ \\
\hline " & IOc orange & 3880 & “ & $388 \mathbf{I}$ \\
\hline “ & $25 \mathrm{c}$ blue & 3880 & “ & $388 I$ \\
\hline Cordova & $5 \mathrm{c}$ brown & $429 I$ & “ & $428 \mathrm{I}$ \\
\hline C. Bravos & $50 \mathrm{c}$ green & 488 & " & 4883 \\
\hline Mexico & IOc orange & 5489 & “، & 5480 \\
\hline “، & $25 \mathrm{c}$ blue & 5489 & "، & 5480 \\
\hline ، & $25 \mathrm{c}$ & 548 & “ & 5481 or 82 \\
\hline ‘. & $25 \mathrm{c}$ " & 542 & . & 5482 \\
\hline
\end{tabular}

\begin{tabular}{|c|c|}
\hline $\begin{array}{cc}\text { Paper. } & \text { Meas. } \\
\text { ? } & ?\end{array}$ & $\begin{array}{l}\text { Sur. } \\
\text { (Heitman's Handbuch) }\end{array}$ \\
\hline$?$ & "“ \\
\hline ord. vert laid & $\begin{array}{l}\text { none (in strip of } 3 \text { ) } \\
\text { none }\end{array}$ \\
\hline thin soft ver. laid & \\
\hline & ${ }^{1} 5 \times 3$ black in pair \\
\hline & I5 \\
\hline $\begin{array}{l}\text { ord. vert. laid } \\
\text { horizontal laid }\end{array}$ & I $8 \times 21 / 4$ vermilion \\
\hline id & “ \\
\hline ert. laid & (strip of 3 ) \\
\hline ord. & “ \\
\hline ord. ver. laid & " " \\
\hline $\begin{array}{l}\text { thin soft vert. laid } \\
\text { very thin wove }\end{array}$ & $\begin{array}{l}\text { I } 4 \times I \text { I } 3 / 4 \text { black } \\
\text { none }\end{array}$ \\
\hline ? & $\begin{array}{l}\text { IIxI } 7 / 8 \text { black } \\
\text { (Heitmans Handbouch.) }\end{array}$ \\
\hline $\begin{array}{l}\text { ord. wove } \\
\text { thin soft vert laid } \\
\text { ? }\end{array}$ & $\begin{array}{c}\text { IIxI } 7 / 8 \text { black } \\
\text { ". } \\
\text { (Heitmans }\end{array}$ \\
\hline
\end{tabular}

Surcharge of No. and Date inverted.

\begin{tabular}{|c|c|}
\hline & \\
\hline $\begin{array}{l}\text { District. } \\
\text { Vera Cruz }\end{array}$ & Value. Color. \\
\hline I6 $\mathrm{Tab}$ & $5 \mathrm{c}$ brown \\
\hline 54 Mexico & $50 \mathrm{oc}$ green \\
\hline (2 Tepic & IOC green \\
\hline
\end{tabular}

Paper.

ord. wove ord. vert laid ".
Meas. Sur. no surcharge og\& I IXI $7 / 8$ black osts $9 \times I 1 / 2$ " $6 L z I$
Sur. Color. corrected byhand to 38 ?citman's Handbuch corrected byhand to 38 I 4 I $3 / 4$, corrected by figure 4 printed over

(Heitman's Handbuch) (strip of 3 )

corrected by figure 8 over first 9

$$
\begin{aligned}
& \begin{array}{ll}
28 \times 21 / 2 & \text { " } \\
\text { none } & \text { (strip of } 3) \\
\text { I I } 7 \text { I } 7 / 8 \text { black }
\end{array} \\
& \text { "، "، } \\
& \text { "1 } 11 \\
& \text { "، } \\
& \text { a }
\end{aligned}
$$


Surcharge with onc or two of the figures inverted.

\begin{tabular}{|c|c|c|c|c|c|}
\hline & District & Value. & Paper. & Meas. Sur. Co & olor. \\
\hline I & Zamora & $25 \mathrm{c}$ blue & ord. wove & I $3 \times 1 / 2$ violet & $\mathbf{1 7 9}$ \\
\hline 2 & Zacatecas & $25 c " \cdots$ & & I $6 \times 17 / 8$ black & 279 \\
\hline 26 & Merida & $\begin{array}{l}5 \mathrm{c} \text { brown } \\
5 \mathrm{c}\end{array}$ & ord. vert Iaid & $\begin{array}{l}\text { 13x2 black } \\
\text { none }\end{array}$ & $\begin{array}{l}z 680 \\
z 680\end{array}$ \\
\hline & “" & $5 \mathrm{c} \quad ،$ & “ & none & z680 twice \\
\hline & “" & Iooc carmine & ord. wove & none & $z 680$ \\
\hline 54 & Mexico & $50 c$ green & ord. vert laid & 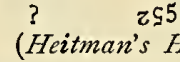 & $\begin{array}{l}\text { 5I instead of } 5482 \\
\text { Handbuch.) }\end{array}$ \\
\hline
\end{tabular}

Two stamps connected, one surcharged with No. and abbreviated date and name of postal district the other with No. and abbreviated date only.

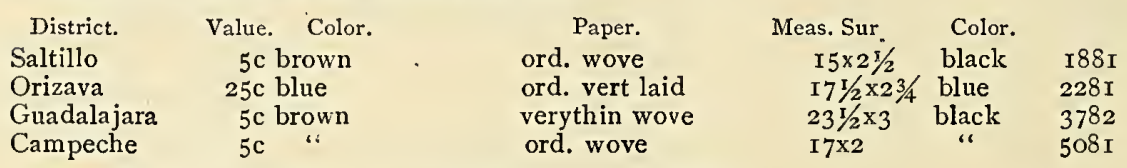

With error in position of number and date and Mexico.

25 $\mathrm{c}$ blue dull brownish red ord. vert laid $\quad$ IIxI7/8 black Surcharged thus:

With incorrect or divided grouping of the figures composing the district number and date.

\begin{tabular}{|c|c|c|c|c|c|c|c|}
\hline $\begin{array}{l}\text { District. } \\
\text { Vera Cruz }\end{array}$ & $\begin{array}{l}\text { Value. } \\
5 \mathrm{c}\end{array}$ & \multicolumn{2}{|c|}{$38 \mathrm{r}$ instead of } & $38 \mathrm{I}$ & $\begin{array}{c}\text { Paper. } \\
\text { ord. wove }\end{array}$ & $\begin{array}{l}\text { Meas. Sur. } \\
?\end{array}$ & $\begin{array}{l}\text { Color. } \\
\text { (Heitman's Handbuch) }\end{array}$ \\
\hline " & IOC & $38 \mathrm{I}$ & & $38 I$ & ord. vert. laid & $15 \times 13 / 4$ & black \\
\hline “ & $25 \mathrm{C}$ & $38 \mathrm{I}$ & “ & $38 \mathrm{I}$ & ord. wove & & “" \\
\hline “ & $25 \mathrm{c}$ & 382 & “ & 382 & very thin wove & $\mathrm{I} 7 \mathrm{I} / 2 \times \mathrm{xr} 3 / 4$ & “" \\
\hline Zacatecas & $25 \mathrm{c}$ & 282 & “" & 282 & “ & $16 \times 17 / 8$ & $\underset{:}{\text { carmine }}$ \\
\hline Tula & $25 \mathrm{c}$ & 782 & “" & 782 & “ & $8 \times 13 / 4$ & “" \\
\hline Tuxpan & $4 \mathrm{c}$ & 883 & “ & 883 & “ & ? & (Heitman's Handbuch) \\
\hline Toluca & IOC & 980 & “ & 980 & ord. wove & ? & (Heitman's Handbuch) \\
\hline Tehuacan & $\begin{array}{r}25 \mathrm{c} \\
5 \mathrm{c}\end{array}$ & 980 & “" & $\begin{array}{r}980 \\
982\end{array}$ & ord, vert. laid & $\frac{13 \times 2}{?}+\frac{1}{2}$ & $\begin{array}{l}\text { blue } \\
\text { (Heitman's Handbuch) }\end{array}$ \\
\hline Tlaxcala & IOC & 382 & “" & 1382 & ord. wove & ? & (Heitman's Handbuch) \\
\hline Tula de $\mathrm{T}$. & $\begin{array}{r}\text { roc } \\
\text { rooc }\end{array}$ & $\begin{array}{r}680 \\
-680\end{array}$ & “ & $\begin{array}{l}680 \\
680\end{array}$ & ord. vert. laid & $\begin{array}{l}\text { I } 5 / 4 \times 13 / 4, \\
\text { none }\end{array}$ & with period; black \\
\hline S. L. Potosi & $25 \mathrm{C}$ & I 782 & “" & I782 & very thin wove & $15+\omega \times 23 / 4$ & black \\
\hline$\underset{6 ،}{\text { Puebla }}$ & IOC & 2082 & “" & 2082 & & $13 \times 2$ & \\
\hline Oaxaca & $\begin{array}{r}25 \mathrm{c} \\
4 \mathrm{c}\end{array}$ & $\begin{array}{l}2083 \\
2380\end{array}$ & “" & $\begin{array}{l}2083 \\
2380\end{array}$ & ord. vert. laid & $\begin{array}{l}13 \times 2 \\
16 \times 2\end{array}$ & "“ \\
\hline “ & rooc & 2380 & “ & 2380 & ord. wove & $13 \times 2$ & with " period; ; \\
\hline “ & IOC & 2383 & “" & 2383 & very thin wove & $20 \times 3 \frac{1}{2}$ & black \\
\hline “ & IOOC & 2383 & “" & 2383 & " & none & \\
\hline Monterey & IOOc & 2583 & “ & 2583 & “" & none & \\
\hline Merida & $25 c$ & 2682 & “، & 2682 & thin soft vert. laid & $\mathrm{r} 3 \times 2$ & black \\
\hline $\begin{array}{l}\text { Monterrey } \\
\text { Lagos }\end{array}$ & $\mathrm{I}_{\mathrm{OC}}$ & $285 \mathrm{I}$ & " & 2581 & $?$ & ? & (Heitman's Handbuch) \\
\hline Lagos & 5 Oc & $3 r^{80}$ & & 3180 & & & (Heitman's Handlbuch) \\
\hline $\begin{array}{l}\text { Jalapa } \\
\text { Parral }\end{array}$ & roc & 3383 & “ & 3383 & very thin wove & I $5 \times 3$ & black \\
\hline & $25 \mathrm{c}$ & $\begin{array}{l}3482 \\
3482\end{array}$ & “. & $\begin{array}{l}3482 \\
3482\end{array}$ & ." & $\begin{array}{c}\text { none } \\
?\end{array}$ & (Heitman's Handbuch) \\
\hline Guanajuato & $25 \mathrm{c}$ & 3668 & ، & 3668 & ? & date is a & $\begin{array}{l}\text { wrong } \\
\text { (Heitman's Handbuch }\end{array}$ \\
\hline $\begin{array}{l}\text { Chiapas } \\
\text { Chalco }\end{array}$ & IOc & $408 r$ & “" & $408 I$ & ord. wove & none & \\
\hline Chalco & IOC & 4 I 83 & & $4 I 83$ & very thin w & none & \\
\hline Mexico & IOC & 2 & .. & 5482 & ord. wove & $\operatorname{IIXI} 7 / 8$ & black \\
\hline
\end{tabular}

With the exception of the last one, all the following have been copied from Heitman's Handbuch.

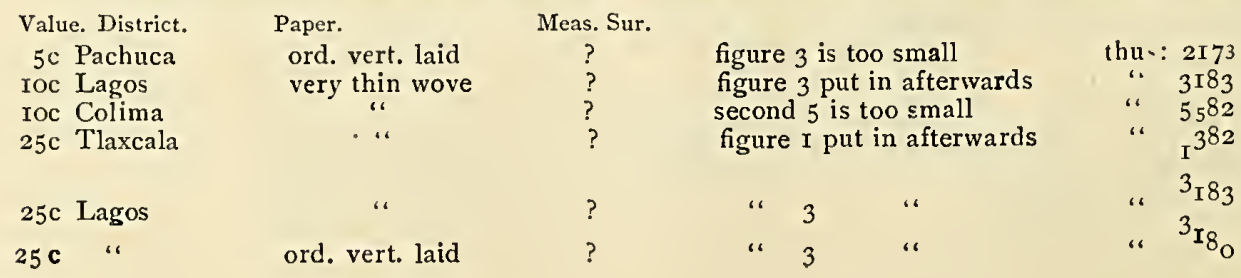






$\begin{array}{lcc}\text { Value. District. } & \text { Paper. } & \text { Meas. Sur. } \\ \text { 25c Guanajuato thin soft wove } & ? \\ \text { 25c Colima } & \text { very thin wove } & ? \\ 50 \mathrm{c} \text { Cordova } & \text { ord. wove } & \text { " } \\ \text { 25c Mexico } & \text { IIXI } 7 / 8\end{array}$

March, 1879.

Three quarter face portrait of President Juarez to the left, engraved on white paper of varıous kinds. This series was used for foreign postage only, the type previously described being, since March, 1879, used for inland postage only.

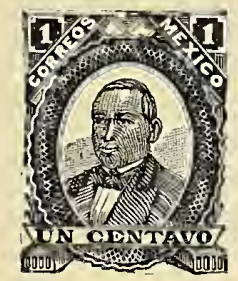

II. Without any surcharge.

Perforated 12.

I $^{\circ}$ On rather thick paper, laid vertically with narrow lines. (1879-82)

I756 Ic brown

I 757 2c dull violet

I758 5c orange

I759 Ioc blue

I760 roc pale blue

I $76 \mathrm{I} 25 \mathrm{c}$ carmine rose

I 762 5Oc olive green

I $76385 \mathrm{c} \mathrm{light}$ purple

I764 rooc black

$2^{\circ}$ On very thin wove paper. (I88I-82)

I765 Ic brown

I 766 2c dull violet

1767 2c slate

I 768 5c orange

I769 5c yellow orange

I770 IOc blue

I77I roc pale blue

$1772 \quad 25 \mathrm{c}$ carmine rose

I773 50c olive green

$1774 \quad 85 \mathrm{c}$ light purple

I775 rooc black

Varieties: Errors of color.

I 776 Ioc pale brown

$177725 \mathrm{c}$ reddish brown

It is very doubtful if any of the above on thin wove paper were ever used for postage in this condition, although some of the laid paper ones are occasionally met with.

II. Surcharged with number and abbreviated date only.

$I^{\circ}$ On rather thick paper, laid vertically with narrow lines. (I879-82)

I 778 $\quad$ Ic brown
I779 $\quad$ 2c dull violet
I780 $5 \mathrm{c}$ orange
I78 I $\quad$ IOc blue
I782 IOc pale blue
I783 25c carmine rose
I784 50c olive green
I785 85c bright purple
I786 IOOc black
20 On thin hard paper, laid vertically
with narrow lines. (I88I-82)
I787 Ic brown

$\begin{array}{lcl}\text { figure } 3 \text { put in afterwards } & \text { thus } & 682 \\ \text { first } 5 \text { is too small } & \text { “ } & 3582 \\ \text { first } 4 \text { wrong type } & \text { “ } & 4278 \\ \text { first } 5 \text { is too small } & \text { " } & 5479\end{array}$

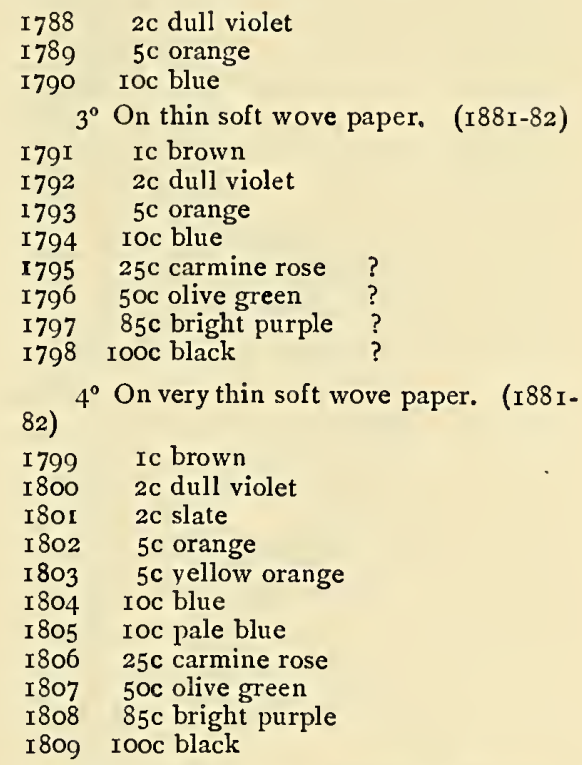

III. Surcharged with number and name of postal district and abbreviated date.

$I^{\circ}$ On rather thick paper, laid vertically with narrow lines. (I88I-82)

I8 Io Ic brown

I8II $2 \mathrm{c}$ dull violet

I I2 5C orange

I 8 I3 Ioc blue

I8I4 Ioc pale blue

I 8 I 5 25c carmine rose

I8I6 50c olive green

I $817 \quad 85 \mathrm{c}$ bright purple

I 8 I 8 rooc black

$2^{\circ}$ On thin hard paper, laid vertically with narrow lines. (I88I-82)

I8I9 Ic brown

I $820 \quad 2 \mathrm{c}$ dull violet

I 82 I $5 \mathrm{C}$ orange

I822 roc blue

$3^{\circ}$ On thin soft wove paper. (I88I-82)

I823 Ic brown

I 824 2c dull violet

I $825 \quad 5 \mathrm{c}$ orange

I 826 IOc blue

I 827 25c carmine rose ?

I 828 5Oc olive green ?

I829 $85 \mathrm{c}$ light purple ?

I830 rooc black

$4^{\circ}$ On very thin wove paper. (I38I-82)

I83I Ic brown

I832 2c dull violet

I 833 2c slate

I834 $5 \mathrm{c}$ orange

I835 5c yellow orange

I 836 Ioc blue

I837 Ioc pale blue

I838 25c carmine rose

I839 50c green

I $840 \quad 85 \mathrm{c}$ bright purple

I 84 I Iooc black 
Names and numbers of Postal Districts of this issue.

\begin{tabular}{|c|c|}
\hline$x 879$ & 1 ZAMORA I3xI $1 / 2$; bluish violet \\
\hline I879-8I & I $3 \times 1 \mathrm{I} / 2 ;$ dark violet \\
\hline I $88 \mathrm{I}-83$ & I $3 \times 1 \mathrm{I} / 2 ;$ black \\
\hline $1876-81$ & 2 ZACATECAS $161 / 2 \times 2$; black \\
\hline I879-8I & I6xI $7 / 8 ;$ black \\
\hline I $879-8$ I & I6x $7 / 8 ;$ blue \\
\hline I882 & $\mathrm{I} 6 \mathrm{I} / 2 \times 2$; vermilion \\
\hline $\mathrm{I} 882-83$ & $161 / 2 \times 2 ;$ carmine \\
\hline $1882-83$ & $16 \times 17 / 8 ;$ carmine \\
\hline I $882-83$ & I61/2 $\times 2$; violet \\
\hline 1879 & 3 VERACRUZ I $8 \times 3-1 \mathrm{x} / 2$; black \\
\hline I $879-8 \mathrm{I}$ & VERACRUZ I $3 \times 2$; black \\
\hline I $879-83$ & VERACRUZ I 7x I $3 / 4$; black \\
\hline I88o-8I & I 5 XI $1 / 2 ;$ black \\
\hline I 883 & VERACRUZ I $71 / 2 \times 21 / 2$ : black \\
\hline I 879 & $7 \times 2$; black \\
\hline $\begin{array}{l}1880 \\
1879-83\end{array}$ & $\begin{array}{rr}\text { HERMOSILLO } & \text { 20x3; violet } \\
\text { “ } & 20 \times 3 ; \text { black }\end{array}$ \\
\hline I879-83 & HERMOSILLO 20x3; black \\
\hline I 879-82 & 5 TULANCINGO I $8 \times 2$; black \\
\hline I $879-82$ & I $8 \times 2 ;$ blue \\
\hline I $882-83$ & TULANCINGO 23×3; black \\
\hline I $879-83$ & $\begin{array}{l}\text { I5 } 1 / 4 \times 13 / 4, \text { with } \\
\text { period; black }\end{array}$ \\
\hline I $879-83$ & $8 \times 13 / 4 ;$ black \\
\hline I $880-8$ I & $8 \times x 3 / 4$; blue \\
\hline I $88 \mathrm{I}-82$ & $8 \times 13 / 4 ;$ carmine \\
\hline I $879-82$ & I I I $5 / 8$; black \\
\hline I $88 \mathrm{I}-82$ & I IxI $5 / 8 ;$ blue \\
\hline I $882 \cdot 83$ & I IXI $5 / 8$; carmine \\
\hline I 882 & IIXI $5 / 8$; brown \\
\hline I 883 & IIXI $5 / 8 ;$ violet \\
\hline $\mathrm{I} 88 \mathrm{I}-82$ & I IX2 $1 / 2 ;$ black \\
\hline I $88 I-82$ & I IX21/2; blue \\
\hline I 882 & I IX2 $1 / 2$; carmine \\
\hline 1879 & 9 TOL UCA $\quad$ I $3 \times 13 / 4$; blue \\
\hline I $879-80$ & $\begin{array}{l}\text { TOLUCA. } \begin{array}{l}\text { I3 } 1 / 2 \times 21 / 2, \text { with } \\
\text { period; blue }\end{array}\end{array}$ \\
\hline I $880-82$ & TOLULA $\quad$ r $43 / 4 \times 3 ;$ blue \\
\hline 1882 & $\mathrm{I} 43 / 4 \times 3$; violet \\
\hline I $882-83$ & $\begin{array}{c}\text { TOLUCA. } \\
\text { violet } \\
\text { vion } 1 / 2 \text {, with period; }\end{array}$ \\
\hline I879-80 & 10 TEHUACAN $151 / 2 \times 17 / 8$; blue \\
\hline I $880-83$ & $\begin{array}{l}\text { I } 51 / 2 \times 17 / 8 ; \text { bright } \\
\text { rose }\end{array}$ \\
\hline I 883 & I $51 / 2 \times 17 / 8 ;$ carmine \\
\hline I 883 & 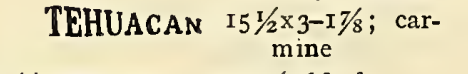 \\
\hline $1879-83$ & 11 TEXCOCO I3XI $1 / 2$; black \\
\hline $879-83$ & 12 TEPIC $9 \times 1 / 2 ;$ black \\
\hline I880 & $9 \times 1 \frac{1}{2} ;$ blue \\
\hline $\mathrm{I} 882$ & $9 \times 1 / 2 ;$ reddish violet \\
\hline I 883 & $7 \times 2$; black \\
\hline I883 & $7 \times 2 ;$ blue \\
\hline $\begin{array}{l}1883 \\
1879-83\end{array}$ & $\begin{array}{l}\text { “ } 7 \times 2 \text { Theddish violet } \\
13 \text { TLAXCALA } 141 / 2 \times 13 / 4 ; \text { blue }\end{array}$ \\
\hline I 879 & $\begin{array}{r}14 \cdot \text { TAMPICO - } \begin{array}{r}\text { I } 1 / 2 \times 2, \text { between } \\
\text { periods; black }\end{array}\end{array}$ \\
\hline
\end{tabular}

\begin{tabular}{|c|c|c|}
\hline I $879-80$ & TAXPICO I & I $51 / 2 \times 2$; black \\
\hline I $880-8 \mathrm{I}$ & “ & I $51 / 2 \times 2$; blue \\
\hline I88 I & “ & I $51 / 2 \times 2$; vermilion \\
\hline I88I & “ & I $51 / 2 \times 2$; violet \\
\hline I $88 \mathrm{I} \cdot 82$ & TAMPICO & I $4 \times 21 / 2-\mathrm{I} 3 / 4 ;$ violet \\
\hline I $882-83$ & & I $4 \times 13 / 4$; black \\
\hline 1882 & TAMPICO & I $8 \times 3$; violet \\
\hline $1882-83$ & “ & I8x3; black \\
\hline I $879-8315$ & 5 TACUBAYA I4 & I $41 / 2 \times$ I $1 / 2 ;$ blue \\
\hline I879-83 16 & 6 TABASC0 14 & $141 / 2 \times 13 / 4 ;$ black \\
\hline $\mathrm{x} 880$ & TABASCO I4 & $\begin{array}{l}\text { I } 41 / 2 \times 21 / 2-11 / 2 ; \\
\text { black }\end{array}$ \\
\hline I879-80 17 & 7 S.L.POTOS! I 5 & $5 \times 53 / 4 ;$ black \\
\hline I $880-83$ & SLPOTOSI $x_{5}$ & I53/4 $\times 23 / 4 ;$ black \\
\hline I $879-8318$ & 8 SALTILLO & I $5 \times 2 \mathrm{~T} / 2 ;$ black \\
\hline I $879-83 \quad 19$ & 9 OUERETARO & I41/2xI 3/4; black \\
\hline 1883 & “ & I $41 / 2 \times 13 / 4 ;$ blue \\
\hline I883 & “ & $\begin{array}{l}\mathrm{I} 4 \mathrm{I} / 2 \times 13 / 4 ; \text { violet } \\
\text { blue }\end{array}$ \\
\hline I $879-8320$ & 0 PUEBLA & I $3 \times 2$ : black \\
\hline I $882-83$ & “ & $\begin{array}{l}\text { 13X2; reddish } \\
\text { violet }\end{array}$ \\
\hline I 882 & “. & I $3 \times 2 ;$ blue \\
\hline I $879-83 \quad 21$ & 21 PACH UCA & I $5 \times$ × $3 / 4 ;$ black \\
\hline I $879-80 \quad 22$ & 2 ORIZAVA. & $\begin{array}{l}\text { I } 7 \times 23 / 4, \text { with } \\
\text { period; blue }\end{array}$ \\
\hline I $879-82$ & ORIZAVA. I & $\begin{array}{l}17 \frac{1}{2} \times 23 / 4 \text {, with } \\
\text { period; blue }\end{array}$ \\
\hline I $879-82$ & ORIZAVA & I $3 \times 13 / 4$; blue \\
\hline I $879-82$ & ORIZAVA & I $4 \times 2$; blue \\
\hline $1882-83$ & $O R I Z A B A$ & $A_{20 \times 31 / 2} ;$ blue \\
\hline I 879 & 3 OAXACA & $\begin{array}{l}\text { name IIX2; } \\
\text { frame I } 1 / 2 \times 3 \frac{\pi}{2} \text {; } \\
\text { all in blue }\end{array}$ \\
\hline I 879-8I & OAXACA & $\begin{array}{l}\text { I6x2, with } \\
\text { period; blue }\end{array}$ \\
\hline I880 & . & $\begin{array}{l}\text { I6x2, with } \\
\text { period; green }\end{array}$ \\
\hline I $880-83$ & ד & $A_{20 \times 31 / 2 ; \text { black }}$ \\
\hline $\mathrm{I} 882-83$ & “ & $20 \times 31 / 2 ;$ carmine \\
\hline I $879-8224$ & 4 MORELIA & I $5 \times 2$; black \\
\hline I $879-82$ & MORELIA & $\begin{array}{l}\mathrm{I} 5 \times \mathrm{x} 3 / 4-2 \mathrm{x} / 2 ; \\
\text { black }\end{array}$ \\
\hline 1882 & MORELIA & I $4 \times 21 / 2 ;$ violet \\
\hline I 882 & MORELIA & I4×23/4; black \\
\hline I $882-83$ & MORELIA & I 7×3\%1/4; black \\
\hline I $882-83$ & MORELIA & $\begin{array}{c}A_{\text {I } 7 \times 3 \pi / 4-41 / 2} ; \\
\text { black }\end{array}$ \\
\hline I $879-8$ I 25 & 5 MONTERREY & YY $\quad 19 \times 2$; black \\
\hline I $88 I-82$ & MONTE & $=Y_{23 \times 31 / 2 ; b l a c k}$ \\
\hline $1882-83$ & & $\begin{array}{l}23 \times 31 / 2 ; \text { car- } \\
\text { mine }\end{array}$ \\
\hline $\mathrm{I} 883$ & " & $23 \times 3 \frac{1}{2} ;$; violet \\
\hline
\end{tabular}





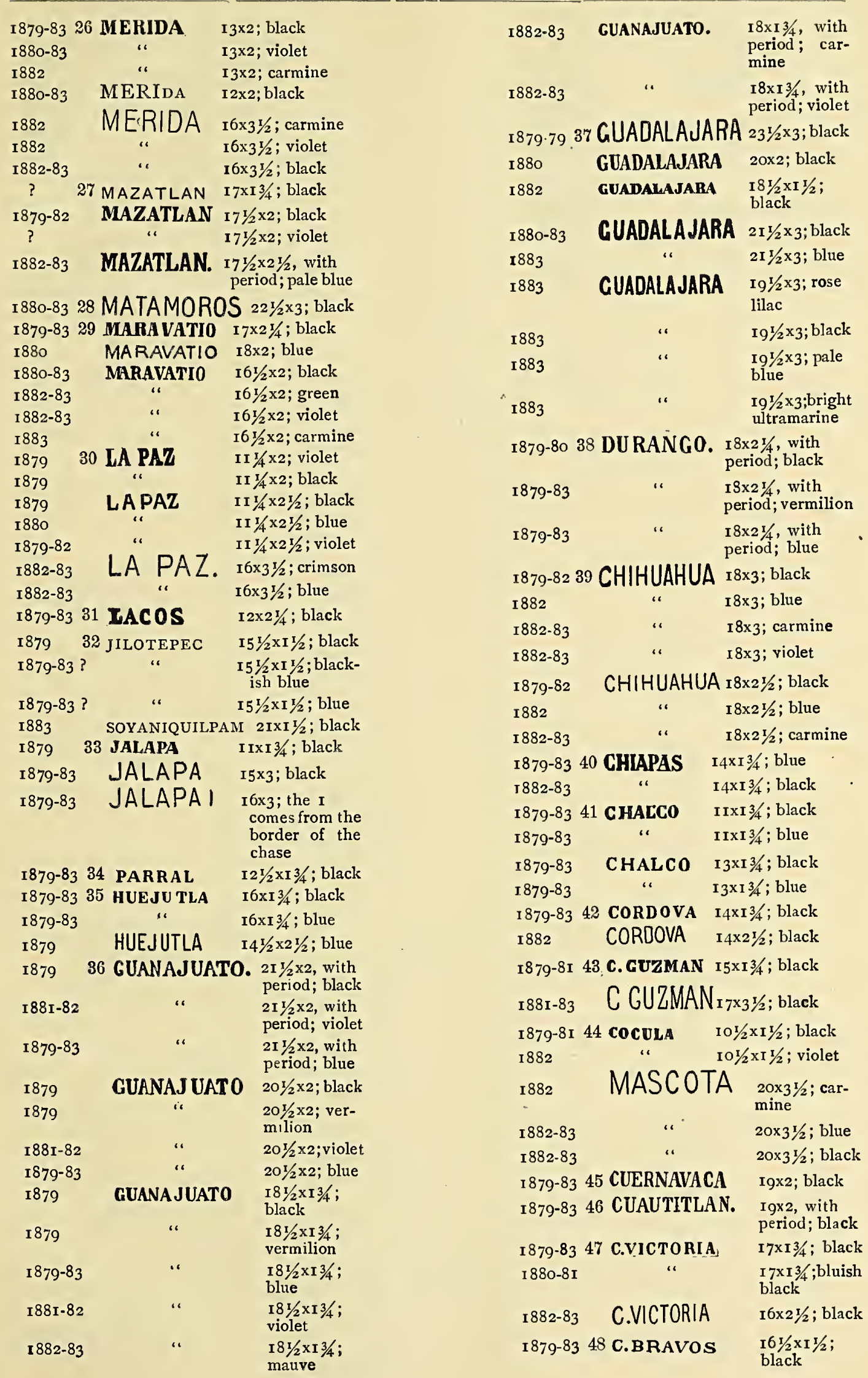




\begin{tabular}{|c|c|c|}
\hline $\begin{array}{l}1879-81 \\
1879-81\end{array}$ & 49 COLIMA & 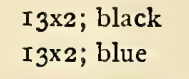 \\
\hline I 879-8I & COLIMA & I3×23/4; black \\
\hline I879-8I & “ & I3×23/4; blue \\
\hline 1882 & COLIMA & I $8 \times 23 / 4$; black \\
\hline I $879-83$ & 50 САMP ECHE & I 7 2 ; black \\
\hline 1883 & & I 7×2; blue \\
\hline $1879-83$ & 51 APAM & $9 \times 2$; black \\
\hline $1879-83$ & & $9 \times 2$; blue \\
\hline I 882 & APA M & I3×3; blue \\
\hline I $882-83$ & “" & 13×3; black \\
\hline
\end{tabular}

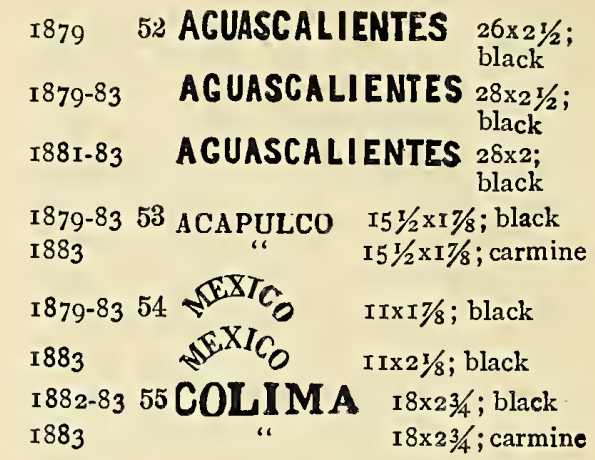

\begin{tabular}{|c|c|c|c|c|c|c|c|}
\hline District. & $\begin{array}{l}\text { No. and } \\
\text { Date. }\end{array}$ & $\begin{array}{l}\text { Surcharge } \\
\text { in }\end{array}$ & $\begin{array}{l}\text { On } \\
\text { Value }\end{array}$ & Color. & District. & $\begin{array}{l}\text { No. and } \\
\text { Date. }\end{array}$ & Paper. \\
\hline (Vera Cruz) & 383 & carmine & IOOC & black & (Chihuahua) & 3679 & ord. vert. laid \\
\hline Toluca & 982 & ، & $2 \mathrm{c}$ & violet & Mexico & 5482 & "40 \\
\hline "، & 982 & “ & $2 c$ & " & " & 5483 & very thin wove \\
\hline “ & 982 & “ & IOC & blue & (Zacatecas) & $\begin{array}{r}5403 \\
282\end{array}$ & vely linis wove \\
\hline “" & 982 & black & IOC & 66 & (") & 282 & "، \\
\hline “ & 982 & carmine & IOC & “ & (Saltillo) & I880 & ord. vert. laid \\
\hline ") & 982 & 11 & IOC & ““ & (“) & I 880 & I" \\
\hline ، & 982 & $\because$ & IOC & $\because 6$ & (Queretaro) & I98 I & “ \\
\hline "، & 982 & “" & IOC & “ & Chihuahua & 3980 & “ \\
\hline " ) & 982 & “ & IOC & 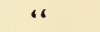 & $(6)$ & 3980 & “ \\
\hline ، & 982 & “ & IOC & “ & Mexico & 5482 & very thin wove \\
\hline (Tepic) & I 283 & “ & $2 \mathrm{C}$ & violet & (Vera Cruz) & 379 & ord. vert. laid \\
\hline 13 & 1283 & “ & $2 \mathrm{c}$ & 6 & ( 6$)$ & 382 & very thin wove \\
\hline “ & 1283 & “ & IOC & blue & (Monterey) & $258 I$ & ord. vert. laid \\
\hline " & i283 & “ & IOC & $" 6$ & Mexico & $548 I$ & 6 \\
\hline Tampíco & 1482 & black & I C & brown & (La Paz) & 3080 & very thin wove \\
\hline$(\because)$ & 1482 & carmine & IC & "، & Mexico & 5482 & " \\
\hline " & 1482 & , & $5 \mathrm{c}$ & orange & (Vera Cruz) & 381 & ord. vert. laid \\
\hline “ & 1482 & “ & $5 \mathrm{c}$ & 16 & (6) & $38 I$ & thin soft vert. laid \\
\hline “ & 1482 & “ & $5 \mathrm{c}$ & “ & “ & 381 & very thin wove \\
\hline “ & 1482 & “ & $5 c$ & “ & (Queretaro) & I98I & " \\
\hline “ & 1482 & “ & $5 \mathrm{c}$ & “ & (Pachuca) & $2 \mathrm{I} 8 \mathrm{I}$ & ord. vert. laid \\
\hline “ & I 482 & “ & $5 \mathrm{c}$ & 16 & (") & 2180 & 6 \\
\hline “ & 1482 & ““ & $5 \mathrm{C}$ & “ & Chihuahua & $398 \mathbf{I}$ & thin hard vert, laid \\
\hline “ & 1482 & $،$ & $5 \mathrm{c}$ & “ & Durango & 3880 & ord. vert. laid \\
\hline Quere taro & I 982 & black & IOC & blue & (Pachuca) & $218 I$ & " \\
\hline (Puebla) & 2082 & carmine & IC & brown & (Tampico) & $148 \mathrm{I}$ & “ \\
\hline " & 2082 & $" 6$ & IC & “6 & Queretaro & I980 & “6 \\
\hline “ & 2082 & “ & IC & “ & ") & I 980 & “ \\
\hline “6 & 2082 & “ & IC & $\cdots$ & Mexico & $548 \mathrm{I}$ & “6 \\
\hline “ & 2082 & black & IC & “ & "1 & $548 I$ & " \\
\hline$($ ( ) & 2082 & carmine & $2 \mathrm{c}$ & violet & ، & 5482 & very thin wove \\
\hline " & 2082 & " & $5 \mathrm{c}$ & orange & (Pachuca) & $2 I 8 I$ & ord. vert. laid \\
\hline " ) & 2082 & “ & $5 \mathrm{c}$ & ${ }^{\prime 0}$ & (Durango) & $388 \mathrm{I}$ & very thin wove \\
\hline "، & 2082 & “ & IOC & blue & (La Paz) & 3080 & ord. vert. laid \\
\hline “ & 2082 & “ & IOC & “ & (Durango) & 3880 & " \\
\hline “ & 2082 & • & IOC & “ & Mexico & 5482 & very thin wove \\
\hline “) & 2082 & “، & IOC & “ & " & 5482 & ac \\
\hline (Monterey) & 2583 & “" & $2 \mathrm{C}$ & violet & Chihuahua & $\begin{array}{l}3408 \\
3981\end{array}$ & ord. vert. laid \\
\hline “ & 2583 & “ & $2 \mathrm{c}$ & “6 & (Campeche) & $508 \mathrm{I}$ & " \\
\hline “ & 2583 & ، & $2 c$ & “ & Mexico & $548 I$ & “ \\
\hline “ & 2583 & “ & $2 \mathrm{C}$ & “ & "، & 5481 & very thin wove \\
\hline " & 2583 & “ & $2 \mathrm{C}$ & “ & (Zacatecas) & 279 & ord. vert. laid \\
\hline 11 & 2583 & “، & $2 c$ & ، & " & 280 & "6 \\
\hline “ & 2583 & “ & $2 \mathrm{c}$ & “ & (Durango) & 3880 & “ \\
\hline (Mazatlan) & 2782 & “ & IC & brown & (La Paz) & 3079 & “ \\
\hline " & 2782 & ، & IC & " & (" $" 1)$ & 3080 & “ \\
\hline “ & 2782 & “ & IC & “ & Mexico & 5482 & very thin wove \\
\hline “ & 2782 & “ & $2 \mathrm{c}$ & violet & Queretaro & I980 & ord. vert. Iaid \\
\hline$"$ & 2782 & “ & $2 c$ & 66 & " ) & 1980 & "6 \\
\hline$(\cdots)$ & 2782 & “ & $2 \mathrm{C}$ & “6 & (La Paz) & 3080 & “ \\
\hline (Mazatlan) & 2782 & carmine & $2 \mathrm{c}$ & violet & Mexico & 5482 & very thin wove \\
\hline “) & 2782 & " & $5 \mathrm{c}$ & orange & (Tula de T.) & 680 & ord. vert. laid \\
\hline “ & 2782 & “، & $5 \mathrm{c}$ & 6 & (Vera Cruz) & $38 I$ & very thin wove \\
\hline “ & 2782 & “ & $5 \mathrm{c}$ & “ & ( 64 & $38 \mathbf{I}$ & thin hard vert. laid \\
\hline “ & 2782 & “ & $5 \mathrm{c}$ & “ & Durango & $388 \mathrm{I}$ & ord. vert. laid \\
\hline “ & 2782 & “ & $5 \mathrm{c}$ & “ & (Chihuahua) & $398 I$ & “" \\
\hline
\end{tabular}




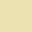





\begin{tabular}{|c|c|c|c|c|c|c|c|}
\hline District. & $\begin{array}{l}\text { No. and } \\
\text { Date. }\end{array}$ & $\begin{array}{l}\text { surcharge } \\
\text { in }\end{array}$ & $\begin{array}{c}\text { On } \\
\text { Value. }\end{array}$ & Color. & District. & $\begin{array}{l}\text { No. and } \\
\text { Date. }\end{array}$ & Paper. \\
\hline (Mazatlan) & 2782 & carmine & $5 \mathrm{c}$ & orange & (Chihuahua) & $398 \mathrm{I}$ & very thin wove \\
\hline ( 63$\}$ & 2782 & "، & roc & blue & (Zaca'ecas) & $28 r$ & ord. vert. laid \\
\hline " & 2782 & “ & IOc & $"$ & (Saltillo) & I 880 & “ \\
\hline$" 6$ & 2782 & “ & roc & “6 & (Huejutla) & $358 \mathrm{I}$ & “ \\
\hline “ & 2782 & “ & IOC & “ & Chihuahua & 3980 & “ \\
\hline “ & 2782 & “ & IOC & “ & $($ ( ) & 3980 & “ \\
\hline (Chihuahua) & 3983 & “ & $2 \mathrm{c}$ & violet & (Vera Cruz) & $38 r$ & “ \\
\hline ( 6$)$ & 3983 & “ & $2 \mathrm{C}$ & "1 & (Monterrey) & $258 \mathrm{I}$ & " \\
\hline Campeche & 5083 & “ & Ic & brown & Merida & 2682 & 6 \\
\hline "1 & 5083 & “ & IC & " & (Guadalajara) & 3782 & very thin wove \\
\hline “ & 5083 & “ & IC & " & 165 & 3782 & "6 \\
\hline “ & 5083 & “ & IC & “ & 'Chihuahua' & 3982 & ord. vert. laid \\
\hline " carmine & 5083 & “، & Ic & “ & "6 & 3982 & " \\
\hline " black & 5083 & “ & rc & “ & Mexico & 5479 & "6 \\
\hline “" & 5083 & “ & $2 \mathrm{c}$ & violet & "1 & 5482 & very thin wove \\
\hline “ & 5083 & “ & $2 \mathrm{c}$ & “ & “ & 5482 & " \\
\hline " & 5083 & “ & $2 \mathrm{c}$ & “ & “ & 5482 & ord. vert. laid \\
\hline “ & 5083 & “ & $5 \mathrm{c}$ & orange & (Queretaro) & rg8I & “6 \\
\hline " carmine & 5083 & “ & roc & , & (Heufulta) & $358 \mathrm{r}$ & “ \\
\hline "6 & 5083 & “ & $5 \mathrm{c}$ & “ & (Cuautitlan) & 4680 & “ \\
\hline “ & 5083 & “ & roc & blue & (Saltillo) & I880 & “ \\
\hline “ & 5083 & “ & roc & $" ،$ & (") & r88I & “ \\
\hline “ & 5083 & “ & roc & ‘ & “ & $r 880$ & “ \\
\hline ( 6 & 5083 & “ & IOc & "، & $(" 1)$ & I88I & “6 \\
\hline Mexico & 5482 & black & IC & brown & (Zactecas) & 280 & “ \\
\hline 6 & 5482 & "6 & $\mathrm{rc}$ & “ & (Saltillo) & r88r & “ \\
\hline ، & 5483 & “ & IC & “ & Tampico & 1481 & “6 \\
\hline “ & 5483 & “ & Ic & “ & Merida & 2682 & very thin wove \\
\hline “ & 5483 & “ & $\mathrm{rc}$ & “ & (Guadalajara) & 3782 & ord. vert. laid \\
\hline “ & 5483 & “ & IC & “ & (Durango) & $388 \mathrm{r}$ & " \\
\hline “6 & 5482 & “ & $\mathrm{rc}$ & “ & (Chihuahua) & 3982 & ، \\
\hline “ & 5482 & “6 & Ic & “ & Mexico & $548 \mathrm{r}$ & ، \\
\hline " ) & 5482 & “ & IC & “ & "I & $548 \mathrm{I}$ & “ \\
\hline " & 5483 & “ & Ic & “ & “ & $548 \mathrm{r}$ & "6 \\
\hline “" & 5482 & 66 & IC & “ & “ & $\begin{array}{l}458 \mathrm{r} \\
\text { error in }\end{array}$ & No. \\
\hline "6 & 5482 & ‘. & Ic & “ & “ & $\begin{array}{l}848 r \\
\text { error in }\end{array}$ & No. \\
\hline “ & 5483 & " & IC & “ & Toluca & 982 & very thin wove \\
\hline “ & 5483 & “ & roc & blue & (Tula de T.) & 680 & ord. vert. laid \\
\hline 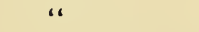 & 5483 & “ & roc & " & (Saltillo) & I880 & 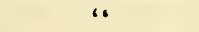 \\
\hline “ & 5485 & “ & I0s & brown & Orzava & 2282 & “ \\
\hline
\end{tabular}

ERRORS.

With name of postal district surcharged two or more times.

\begin{tabular}{|c|c|c|c|c|c|c|c|c|}
\hline $\begin{array}{l}\text { Val. } \\
2 \mathrm{c}\end{array}$ & $\begin{array}{l}\text { Color. } \\
\text { violet }\end{array}$ & $\begin{array}{l}\text { District. } \\
\text { Tepic }\end{array}$ & $\begin{array}{l}\text { Meas. of } \\
\text { Sur. } \\
9 \times 1 / 2\end{array}$ & $\begin{array}{l}\text { Color. } \\
\text { black }\end{array}$ & $\begin{array}{c}\text { No. and } \\
\text { Date, } \\
\mathbf{1 2 8 2}\end{array}$ & $\begin{array}{l}\text { Paper. } \\
\text { very thin wove }\end{array}$ & $\begin{array}{c}\text { Surcharged } \\
\text { twice }\end{array}$ & \\
\hline $5 \mathrm{c}$ & ora & $\mathrm{Mel}$ & $13 \times 2$ & "6 & 26 & & & (Heitman's Handbuch) \\
\hline IOC & blue & & $13 \times 2$ & “" & $26 ?$ & ord. vert. laid & 3 times & 6 \\
\hline Ic & brown & Pue & $\mathrm{r} 2 \times \mathrm{x} 3 / 4$ & “6 & $20 ?$ & " & twice & “ \\
\hline roc & blue & alajara & $2 I I / 2 \times 3$ & “ & 3780 & “ & "6 & \\
\hline Ic & brown & Chiapas & $14 \times 13 / 4$ & blue & $40 ?$ & “" & “ & (Ileitman's Handbuch) \\
\hline
\end{tabular}

Errors in surcharge of number.

\begin{tabular}{|c|c|c|c|c|c|}
\hline $\begin{array}{l}\text { District. } \\
\text { Pachuca }\end{array}$ & $\begin{array}{l}\text { Value. } \\
\text { roc }\end{array}$ & $\begin{array}{l}\text { Color. } \\
\text { blue }\end{array}$ & \multicolumn{3}{|c|}{4282 instead of $2 r 82$} \\
\hline Mazatlan & rc & brown & $478 r$ & ‘ & $278 r$ \\
\hline ، & $5 \mathrm{c}$ & orange & $478 \mathrm{I}$ & 6 & $278 \mathrm{r}$ \\
\hline “ & IOC & blue & 4780 & ‘ & 2780 \\
\hline “ & roc & $" ،$ & $478 r$ & ‘ & $278 r$ \\
\hline “ & $25 \mathrm{c}$ & carmine & $478 \mathrm{I}$ & ‘ & $278 I$ \\
\hline “ & $25 \mathrm{c}$ & "، & 8879 & ‘ & 3879 \\
\hline Chihuahua & $50 \mathrm{c}$ & green & \multicolumn{3}{|c|}{ r 479 instead of 3979} \\
\hline Cordova & $\mathrm{rc}$ & brown & 379 & “" & 4279 \\
\hline Mexico & IC & " & 4579 & “ & 5479 \\
\hline ، & Ic & “ & 4580 & 66 & 5480 \\
\hline “ & $2 c$ & violet & 4580 & “ & 5480 \\
\hline ، & $2 \mathrm{c}$ & “ & 4582 & "6 & 5482 \\
\hline “ & $2 \mathrm{c}$ & "، & 4582 & " & 5482 \\
\hline ، & $2 \mathrm{C}$ & “ & $848 z$ & “ & 5482 \\
\hline " & $5 \mathrm{c}$ & orange & 4579 & $"$ " & 5479 \\
\hline “" & $5 \mathrm{c}$ & $"$ & 4580 & " & 5480 \\
\hline “ & $5 \mathrm{c}$ & “ & $458 \mathrm{r}$ & “" & $548 \mathrm{r}$ \\
\hline “ & $5 \mathrm{c}$ & “ & 4582 & "، & 5482 \\
\hline
\end{tabular}

\begin{tabular}{|c|c|}
\hline Paper. & Meas. of Sur. \\
\hline $\begin{array}{l}\text { very thin wove } \\
\text { ord. vert. laid }\end{array}$ & $\begin{array}{l}r 5 \times r 3 / 4 \\
r 71 / 2 \times 2\end{array}$ \\
\hline “ & “ \\
\hline “ & “6 \\
\hline “ & “ \\
\hline " & $18 \times 21 / 4$ \\
\hline ord. vert. laid & $18 \times 3$ \\
\hline “ & $\operatorname{IrxI} 7 / 8$ \\
\hline “، & "6 \\
\hline “ & 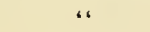 \\
\hline " & 4 \\
\hline very thin wove & “ \\
\hline ord. vert. laid & “، \\
\hline " & “. \\
\hline “ & “ \\
\hline very thin wove & " \\
\hline
\end{tabular}


MEXICO.

\begin{tabular}{|c|c|c|c|c|c|}
\hline Mexico & \multicolumn{2}{|c|}{$5 \mathrm{c}$ violet } & \multicolumn{3}{|c|}{$848 \mathrm{I}$ instead of $548 \mathrm{I}$} \\
\hline “" & IOC & blue & 4580 & “" & 5480 \\
\hline ." & IOC & "“ & $45^{8} \mathrm{r}$ & "، & $548 \mathrm{r}$ \\
\hline ، & IOC & “" & 8482 & “ & 548 \\
\hline " & $25 \mathrm{C}$ & carmine & 4580 & " & 5480 \\
\hline “ & $50 \mathrm{c}$ & green & 4580 & ‘ & 54 \\
\hline “ & $50 \mathrm{c}$ & "6 & 5548 & & 5480 \\
\hline
\end{tabular}

$\begin{array}{cc}\text { ord. vert. laid } & \operatorname{Irx} 7 / 8 \\ \text { ", } & \text { ", } \\ \text { "، } & \text { ", } \\ \text { ", } & \text { ", }\end{array}$

In this stamp the date is also wrong.

Errors in surcharge of date.

\begin{tabular}{|c|c|c|c|c|c|c|c|}
\hline $\begin{array}{l}\text { District. } \\
\text { Merida }\end{array}$ & $\begin{array}{l}\text { Value. } \\
\text { roc }\end{array}$ & $\begin{array}{l}\text { Color. } \\
\text { blue }\end{array}$ & \multicolumn{3}{|c|}{2628 instead of 2682} & $\begin{array}{l}\text { Paper. } \\
\text { very thin wove }\end{array}$ & $\begin{array}{c}\text { Meas, of Sur. } \\
?\end{array}$ \\
\hline Colima & IOC & " & 4928 & “" & 4982 & (3) & ? \\
\hline Acapulco & $50 \mathrm{c}$ & green & 5328 & ، & 5382 & ord. vert. laid & I $5 \mathrm{I} / 2 \mathrm{XI} 7 / 8$ \\
\hline Mexico & Ic & brown & 548 & “" & $5480-81$ or 82 & ") & $\operatorname{IIXI} 7 / 8$ \\
\hline " & IC & " & 5489 & “ & 5480 & “ & 6. \\
\hline 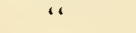 & $2 \mathrm{c}$ & violet & 548 & “ & $5480-81$ or 82 & very thin wove & “ \\
\hline ، & $5 \mathrm{c}$ & orange & 548 & ، & $5480-81$ or 82 & 66 & “" \\
\hline$،$ & IOC & blue & 548 & ، & 5480 or or 82 & “ & 4 \\
\hline “ & IOC & 6 & 5487 & “، & $548 \mathrm{r}$ & ord. vert. laid & “ \\
\hline “ & $25 \mathrm{c}$ & carmine & 5489 & “ & 5480 & “ & “ \\
\hline
\end{tabular}

Errors in position of figures composing number and date.

$\begin{array}{lrrrrr}\text { District. } & \text { Value. } & \text { Color. } & & \text { Paper. } & \text { Meas. of Sur. } \\ \text { Mazatlan } & \text { Ic brown } & 0278 \text { instead of } 2780 & \text { ord. vert. laid } & \text { I } 71 / 2 \times 2 \\ \text { Mexico } & \text { 5c orange } & 7954 & \text { " }\end{array}$

Number and date printed two or more times.

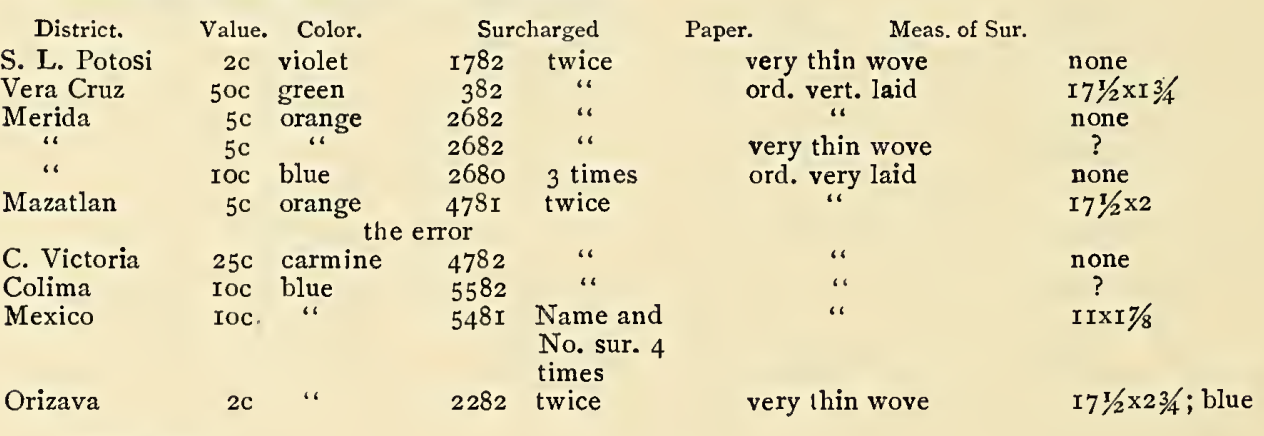

Other errors.

$\begin{array}{crll}\text { District. } & \text { Value. } & \text { Color. } & \\ \text { Mexico } & \text { 2c } & \text { violet } & 5479 \\ \text { “" } & \text { IOc } & \text { blue } & 5482 \\ \text { " } & \text { 2C } & \text { violet } & 5480\end{array}$

July, I882.

Type same as last issue, printed on very thin wove paper.

Perforated 12.

I. Without any surcharge. (1882-83.)

I 842 I2C chestnut brown

I 843 I8c reddish brown

I844 $24 \mathrm{c}$ violet

I 845 5oc pale yellow

I846 rooc orange

It is doubtful if any were used in this condition.

II. Surcharged with number and abbreviated date only. (I882-83.)

r 847 I $2 \mathrm{c}$ chestnut brown

I848 I8c reddish brown

I849 24c violet

I $850 \quad 50 \mathrm{c}$ pale yellow

I85I IOoc orange

III. Surcharged with name and number of postal district and abbreviated date, (1882-83.)

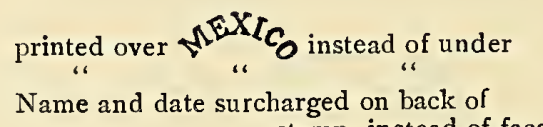

Paper.

ord. vert. laid very thin wove ord. vert. laid stamp, instead of face 


\section{.}



NAmes And NUmbers of Postal Districts of this issue.

\section{ZAMORA \\ 2 ZACATECAS " “" \\ 3 VERACRUZ VERACRUZ \\ 4 HERMOSILLO HERMOSILLO 5 TULANCINCO}

TULANCINGO 6 TULA DE $\mathrm{T}$.

\section{TULA}

8 TUXPAN

".

".

“c

TUSPAN

"“

9 TOLUCA

TOLUCA.

10 TEHUACAN

\section{TEHUAGAN \\ 11 TEXCOCO \\ 12 TEPTC}

TEPIC

13 TLAXCALA

14 TAMPICO

TAMPICO

15 TACUBAYA

16 TABASCO

17 SLPOTOS!

18 SALTILLO

19 OUERETARO "

20 PUEBLA<smiles>[Mg]</smiles>

21 PACHUCA

22 ORIZAVA.

orizava

ORIZAVA

ORIZABA 23 OAXACA
I $3 \times I / 2$; black

I $61 / 2 \times 2$; vermilion

$161 / 2 \times 2$; carmine

I $6 \times 17 / 8$; carmine

I $61 / 2 \times 2$; violet

I $7 / 2 \times 13 / 4$; black

I $71 / 2 \times 21 / 2$; black

20x3; black

20x3; black

I8x2; black

I $8 \times 2$; blue

$23 \times 3$; black

I5 $1 / 4 \times 13 / 4$, with period

black

$8 \times 13 / 4$; black

$8 \mathrm{xI} 3 / 4$; carmine

IIXI $5 / 8$; black

I IxI $5 / 8$; blue

IIXI $5 / 8$; carmine

IIXI $\% / 8$; brown

IIXI $5 / 8$; violet

IIX2 $1 / 2$; black

II $2 \frac{1}{2}$; blue

I IX $2 \frac{1}{2}$; carmine

I $4 \frac{3}{4} \times 3$; blue

$\mathrm{I} 43 / 4 \times 3$; violet

I $5 \times 21 / 2$, with period; violet

$15 \mathrm{I} / 2 \times 17 / 8$; bright rose

I $51 / 2 \times 17 / 8$; carmine

$151 / 2 \times 3-17 / 8$; carmine

I $3 \times 1 \mathrm{I} / 2$; black

$9 \times 11 / 2$; black

$9 \times 1 \mathrm{I} / 2 ;$ reddish violet

$7 \times 2$; black

$7 \times 2$; blue

$7 \times 2$; reddish violet

I $41 / 2 \times 13 / 4 ;$ blue

I $4 \times 13 / 4$; violet

I $4 \times 13 / 4$; black

I $8 \times 3$; violet

I8x3; black

I $41 / 2 \times I 1 / 2 ;$ blue

I $41 / 2 \times 13 / 4$; black

I $53 / 4 \times 23 / 4$; black

I5 $121 / 2$; black

I $41 / 2 \times 13 / 4$; black

I $41 / 2 \times 13 / 4$; blue

$\mathrm{I} 4 \mathrm{I} / 2 \times \mathrm{I} 3 / 4$; violet blue

13x2; black

I3X2; reddish violet

I $3 \times 2$; blue

I $5 \times 13 / 4$; black

[ $71 / 2 \times 23 / 4$, with period;

blue

I $3 \times 13 / 4$; blue

I $4 \times 2$; blue

$20 \times 3 \frac{1}{2} ;$ blue

$20 \times 3 \frac{1}{2}$; black

$20 \times 3 \frac{T}{2}$; carmine

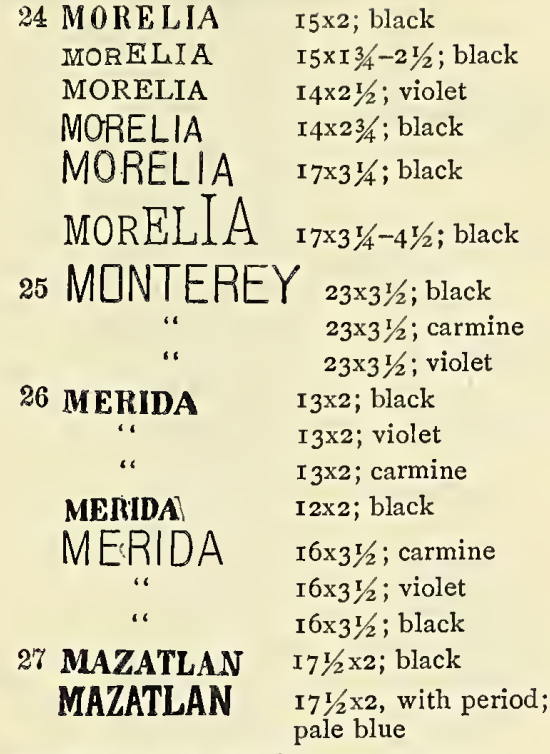

28 MATAMOROS 22 $1 / 2 \times 3$; black

29 MARAVATI0 I $7 \times 2 \frac{1}{4}$; black

MARAVATI0 I6 $1 / 2 \times 2$; black " $\quad 161 / 2 \times 2$; green “ $\quad 161 / 2 \times 2$; violet

" $161 / 2 \times 2$; carmine

30 LAPAZ II $1 / 4 \times 21 / 2$; violet

LA PAZ. I6 $3 \frac{1}{2} / 2$; crimson

$16 \times 3 \frac{1}{2}$; blue

32 JILOTEPEC I5 $1 / 2 \times 1 / 2$; blackish blue SOYANIQUILPAM 2 IXI $1 / 2$; black

33 JALAPA I5 33 ; black

JALAPA I I6x3; the $I$ comes from

34 PARRAL $\quad$ I2 $1 / 2 \times 13 / 4$; black

35 HUEJU TLA I $6 \times 13 / 4$; black

“ I6xI $3 / 4$; blue

36 GUANAJUATO. $2 \mathrm{I} 1 / 2 \times 2$, with period; violet

GUANAJ UATO $201 / 2 \times 2$; violet 2 I $1 / 2 \times 2$, with period; blue

GUANAJUATO I $8 \mathrm{I} / 2 \times \mathrm{xI} / 4$; violet " I $81 / 2 \times 13 / 4 ;$ mauve “ $\quad$ I $81 / 2 \times 13 / 4$; blue

GUANAJUATO. I $8 \times 13 / 4$, with period; carmine $\mathrm{I} 8 \mathrm{xI} 3 / 4$, with period; violet

37 GUADALAJABA I $81 / 2 \times 1 \frac{1}{2} ;$; black

CUADALAJARA $21 \frac{1}{2} \times 3$; black $2 I \frac{1}{2} \times 3$; blue

GUADALAJARA $191 / 2 \times 3$; rose lilac “ $\quad 191 / 2 \times 3$; black " I9 $1 / 2 \times 3$; pale blue

. $\quad 191 / 2 \times 3$; bright ultramarine 


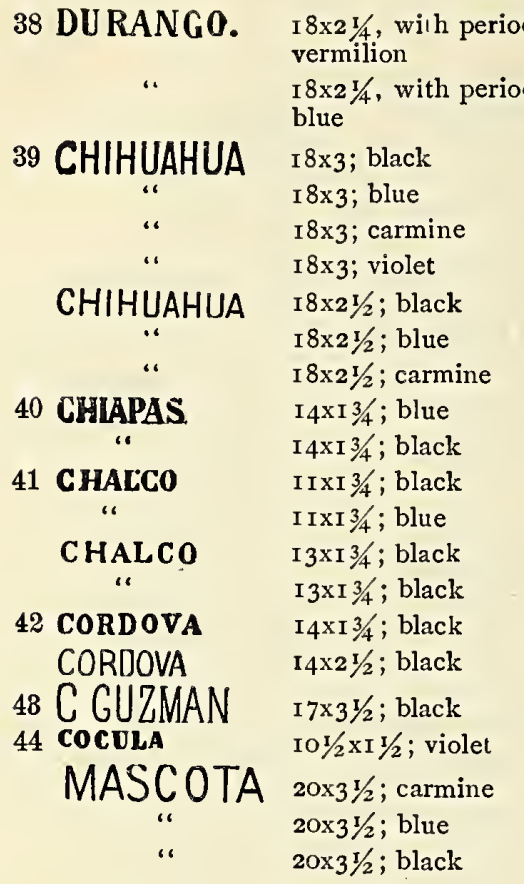

\begin{tabular}{|c|c|c|}
\hline & CUERNAVACA & I9x2; black \\
\hline & CUAUTITLAN. & $\begin{array}{l}\text { I9x2, with period; } \\
\text { black }\end{array}$ \\
\hline 47 & C.VICTORIA & I 7XI 3/4; black \\
\hline & C.VICTORIA & I $6 \times 21 / 2$; black \\
\hline 48 & C.BRAVOS & I6I $1 / 2 \times 11 / 2$; black \\
\hline$\pm \theta$ & COLIMA & I $8 \times 23 / 4 ;$ black \\
\hline 00 & CAMP ECHE & $\begin{array}{l}\text { I } 7 \times 2 \text {; black } \\
\text { I } 7 \times 2 \text {; blue }\end{array}$ \\
\hline 51 & APAM & $9 \times 2$; black \\
\hline & “ & $9 \times 2$; blue \\
\hline & APA & I $3 \times 3$; blue \\
\hline & “" & I 3X2; black. \\
\hline \multicolumn{3}{|c|}{$\begin{aligned} 2 \text { AGUASCALIENTES } & 28 \times 2 \mathrm{1} / 2 ; \text { black } \\
\text { AGUASCALIENTES } & 28 \times 2 ; \text { black }\end{aligned}$} \\
\hline 53 & ACAPULCO & $\begin{array}{l}\text { I5 } 1 / 2 \times 17 / 8 ; \text { black } \\
\text { 151/2 } \times 17 / 8 ; \text { carmine }\end{array}$ \\
\hline & $S^{S X T C_{0}}$ & I IXI $7 / 8$; black \\
\hline & & I I X2 I/8; black \\
\hline & COLIMA & $\begin{array}{l}\text { I } 8 \times 23 / 4 \text {; black } \\
\text { I } 8 \times 23 / 4 \text {; carmine }\end{array}$ \\
\hline
\end{tabular}

HABILITADOS.

$\begin{array}{ccc}\text { District. } & \text { Value. Color. } \\ \text { Jalapa } & 3383 \quad \text { I } 8 \mathrm{c} \text { red brown }\end{array}$

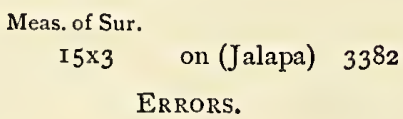

Errors in numbers.

\begin{tabular}{|c|c|c|c|c|c|}
\hline District. & Value. Color. & \multicolumn{4}{|c|}{ Meas. of Sur. } \\
\hline $\begin{array}{l}\text { Campeche } \\
\text { Mexico }\end{array}$ & I 2c brown & 5580 & ead of 5080 & $17 \times 2$ & \\
\hline Mexico & I2C “" & 483 & 5483 & $\operatorname{IXXI} 7 / 8$ & \\
\hline “، & I $8 \mathrm{c}$ red brown & 483 & 5483 & “ & \\
\hline “ & $\begin{array}{l}\text { 24c violet } \\
\text { I2c brown }\end{array}$ & $\begin{array}{r}483 \\
1851\end{array}$ & $\begin{array}{l}5483 \\
548 I\end{array}$ & ““ & No and date both wrong \\
\hline
\end{tabular}

Errors in position of figures composing number and date.

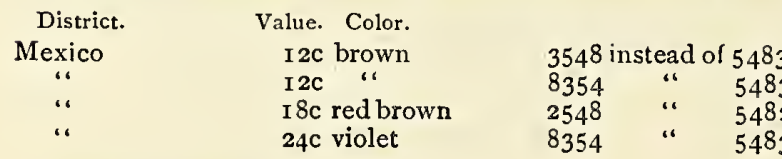

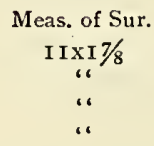

Errors in date.

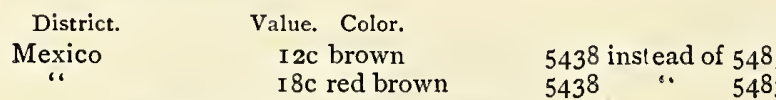

Number and date printed twice.

District.
Tepic
Jalapa

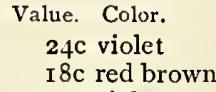

$24 \mathrm{c}$ violet

1282
3382
5482

With number only.
36

$$
\begin{gathered}
\text { Meas. of Sur. } \\
\text { IIXI } 7 / 8
\end{gathered}
$$

$$
\begin{gathered}
\text { Meas, of Sur. } \\
? \\
15 \times 3 \\
11 \times 17 / 8
\end{gathered}
$$

Meas. of Sur. I $81 / 2 \times 13 / 4 \quad$ violel

Other errors.

$\begin{array}{ll}\text { District. } & \text { Value. Color. } \\ \text { Guadalajara } & \text { I2c brown } \\ \text { Hermosillo } & \text { I2c “ } \\ \text { Merida } & \text { I2c } "\end{array}$


. 

July $\mathbf{I} 882$.

Engraved on very thin white wove paper.

Perforated 12.

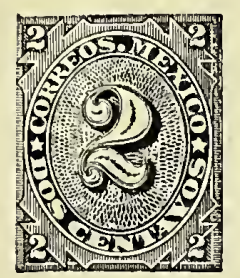

I. Without any surcharge.

(1882 83.)

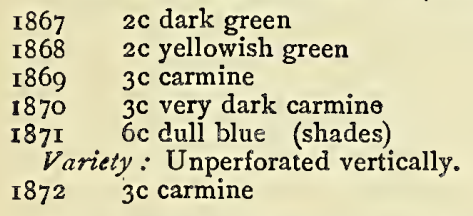

II. Surcharged with number and date only. (1882-83)

$\begin{array}{ll}\text { I873 } & \text { 2c dark green } \\ \text { I874 } & \text { 2c yellowish green } \\ \text { I875 } & \text { 3c carmine } \\ \text { I876 } & \text { 3c very dark carmine } \\ \text { I877 } & \text { 6c dull blue }\end{array}$

III. Surcharged with name and number of postal district and abbreviated date. (I882-83)

$\begin{array}{ll}\text { I878 } & \text { 2c dark green } \\ \text { I879 } & \text { 2c yellowish green } \\ \text { I880 } & \text { 3c carmine } \\ \text { I88 I } & \text { 3c very dark carmine } \\ \text { I882 } & \text { 6c dull blue }\end{array}$

IV. Surcharged with number and date only in a larger type as described before. (1883)

I 883 2c dark green

I884 2c yellow green

I885 3c carmine

I 886 3c very dark carmine

I887 6c dull blue (shades)

I 888 6c bright blue

V. Same as last, with number and date and also name of postal district.

I889 2c dark green

I89o $2 \mathrm{c}$ yellow green

I89 I $3 \mathrm{C}$ carmine

I892 3c very dark carmine

I893 6c dull blue (shades)

I $8946 \mathrm{c}$ bright blue

VI. Surcharged with name of postal district only.
I 895 2C dark green
I896 2c yellow green
I897 3c carmine
$1898 \quad 3 \mathrm{c}$ very dark carmine
I899 6c dull blue
I900 $6 \mathrm{c}$ bright blue

The surcharges on these stamps are the same as those of the preceding issue.

\section{HABILITADOS.}

Value. Color.

3c carmine

$3 \mathrm{c}$ "

$6 \mathrm{c}$ dull blackish blue

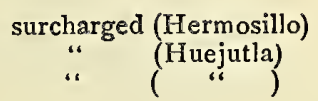

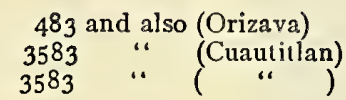

\section{3}

4683

4683 this last No. and date inverled

Errors in numbers.

ERRORS.

\begin{tabular}{|c|c|c|c|c|}
\hline District. & Value. & \multicolumn{3}{|c|}{ Meas. of Sur. } \\
\hline Durango & $2 c$ green & 8883 instead of & 3883 & $18 \times 2$ in vermilion \\
\hline Matamoros & $\begin{array}{l}\text { 3c carmine } \\
6 \mathrm{c} \text { dull blackish blue }\end{array}$ & $\begin{array}{l}7883 \\
7883\end{array}$ & $\begin{array}{l}2883 \\
2883\end{array}$ & $22 \frac{1}{4} \times 3$ in black \\
\hline
\end{tabular}

With number and date inverted.

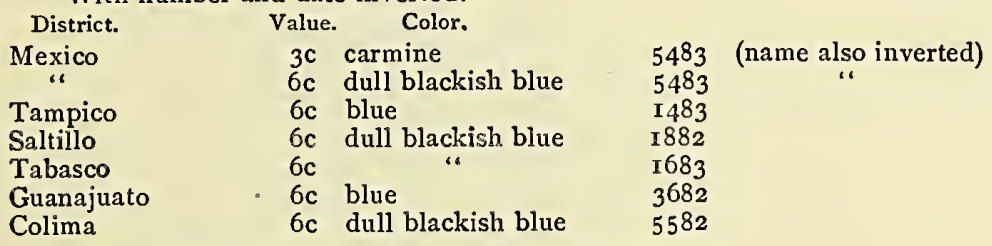

$$
\begin{aligned}
& \text { Meas. of Sur. } \\
& \text { I IXI } 7 / 8 \\
& ? \\
& ? \\
& \text { I5 } 21 / 2 \\
& \text { none } \\
& ? \\
& \text { I } 9 \times 23 / 4
\end{aligned}
$$

\begin{tabular}{|c|c|c|c|}
\hline $\begin{array}{l}\text { District. } \\
\text { Vera Cruz } \\
\text { Tampico }\end{array}$ & $\begin{array}{l}\text { Value. Color. } \\
\text { 3c carmine } \\
\text { 3c }\end{array}$ & $\begin{array}{l}\text { Surcharged } \\
383 \\
\text { I863 } 3 \text { twice } \\
3 \text { times }\end{array}$ & $\begin{array}{l}\text { Meas. of Sur. } \\
\text { none } \\
\text { none }\end{array}$ \\
\hline
\end{tabular}

Number and date surcharged two or more times.

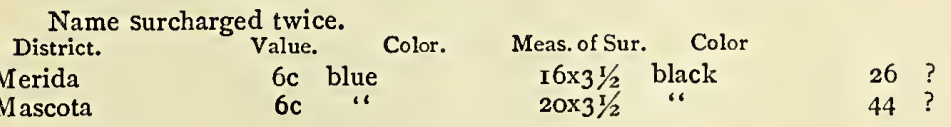

Other Errors.

\footnotetext{
District.

Value. Color.

Mexico

$2 c$ green

5483 Number and Date surcharged above Mexico instead of below
}

Of stamps surcharged with name of postal dislrict only the following are known, of which we have seen he first four 


\begin{tabular}{|c|c|c|c|c|}
\hline Val. & Color. & District. & \multicolumn{2}{|c|}{ Meas. of Sur. Color. } \\
\hline $2 \mathrm{c}$ & green & Mexico & $\operatorname{IIXI7/8}$ & black \\
\hline $3 c$ & carmine & Oaxaca. & $20 \times 31 / 2$ & “ \\
\hline $6 c$ & blue & Toluca & $15 \times 21 / 2$ & violet \\
\hline $6 c$ & $“$ & Chiapas & $\mathrm{I} 4 \mathrm{XI} 3 / 4$ & blue \\
\hline$?$ & ? & Guadalajara & $?$ & ? \\
\hline ? & ? & Jalapa & $?$ & $?$ \\
\hline ? & ? & Mazatlan & ? & ? \\
\hline ? & ? & Merida & ? & ? \\
\hline
\end{tabular}

(Heilman's Handbuch)

January, I884.

Engraved on various papers, size varying in height Irom $211 / 3$ to $22 \mathrm{~mm}$., and in width from $261 / 2$ to $271 / 3 \mathrm{~mm}$. Beginning with 1 his issue, and in accordance with the decree of Oct. Ist, I883, revoking that of August, I87I, in virtue of which stamps had circulation only by their respective districts, the surcharge of the name of the postal district was dispensed with. However, stamps of this and subsequent is:ues are found with surcharge, some of these undoubtedly caused by the postmaster's habit of surcharging stamps (habit becoming second nature), but we cannot understand why surcharges of names should be found which are unknowu on the preceding issues. It is said that as the various postmasters were to surcharge the official stamps with the name of the office, they may sometimes by error have surcharged some of the regular stamps. We have, however, never seen an official stamp with surcharge, although thousands of cancelled ones have passed through our hands. Still, the regular stamps with these new surcharges exist, and those we have seen were undoubtedly genuine.

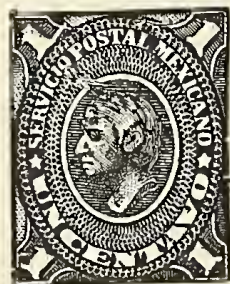

I Th in transparcht papei, laid hoi izontally. Size $211 / 2 \times 27$ to $22 \times 271 / 3 \mathrm{~mm}$.

Perforated 12.

\begin{tabular}{|c|c|}
\hline 901 & Ic green \\
\hline 1902 & Ic yellow green \\
\hline 1903 & Ic bronze green \\
\hline 1904 & Ic blue green \\
\hline I90 & $2 \mathrm{c}$ green \\
\hline & $2 c$ yellow green \\
\hline$[907$ & $2 \mathrm{c}$ bronze green \\
\hline & 2c blue green \\
\hline I 909 & 3c green \\
\hline 9 & 3c yellow green \\
\hline I I & $3 \mathrm{c}$ bronze green \\
\hline I9I 2 & $3 \mathrm{c}$ blue green \\
\hline I 3 & $4 c$ green \\
\hline $\mathrm{I}$ & 4c yellow green \\
\hline I9I6 & $4 \mathrm{c}$ bronze green \\
\hline 8 & 4c sage green \\
\hline I918 & $5 \mathrm{c}$ green \\
\hline I9I9 & $5 \mathrm{c}$ yellow green \\
\hline I920 & $5 \mathrm{c}$ bronze green \\
\hline $\begin{array}{l}1921 \\
1922\end{array}$ & 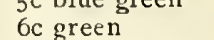 \\
\hline 1923 & $6 \mathrm{c}$ yellow green \\
\hline I 924 & $6 \mathrm{c}$ bronze green \\
\hline & $6 \mathrm{c}$ blue green \\
\hline I926 & IOC green \\
\hline 1927 & IOc yellow green \\
\hline & bronze \\
\hline & I 2c green \\
\hline
\end{tabular}

I93I I2c yellow green

I932 I2c bronze green

I933 I2c blue green

I934 $20 \mathrm{c}$ green

I935 20c yellow green

I936 $20 \mathrm{c}$ bronze green

I937 $20 \mathrm{c}$ blue green

$193^{8} 25 \mathrm{c}$ green

I939 25c yellow green

I $94025 \mathrm{c}$ blue green

I94I $50 \mathrm{C}$ green

I942 50c yellow green

I9+3 Ip blue

I944 $2 \mathrm{p}$ blue

I945 $5 \mathrm{p}$ blue

I9 16 Iop blue

Varieties:

a. Unperforated.

7194 Ic green

I948 3c green

I949 4c green

1950 IOc green

Ihe Ic, 4c, and IOc which we have seen were in entire sheets, ungummed, and probably proofs. Of the $3 \mathrm{c}$ we have seen an unsevered pair, fully gummed and having all the appearances of a regular issue.

b. Unperforated vertically.

I950a $25 \mathrm{c}$ yellow green

$2^{\circ}$ Thin transparent wove paper, varying in thickness. Size $21 \frac{1}{2} \times 27$ to $22 \times$ $27 \mathrm{r} / 3 \mathrm{~mm}$.

Perforated I2.

I95I Ic green

I952 Ic yellow green

I953 Ic bronze green

I954 Ic blue green

I955 2c green

I956 2c yellow green

I957 2c bronze green

I958 2c blue green

I959 3c green

I960 $3 \mathrm{c}$ yellow green

I96I $3 \mathrm{c}$ bronze green

I 962 3c blue green

I963 4c green

I964 4c yellow green

I $9654 \mathrm{c}$ bronze green

I966 4c sage green

$1967 \quad 5 \mathrm{c}$ green

I968 5c yellow green

I969 5c bronze green

I970 $5 \mathrm{c}$ blue green

I97 I 6c green

I972 6c yellow green

I973 6c bronze green

I $9746 \mathrm{c}$ blue green

I975 IOc green

I976 Ioc yellow green

1977 IOc bronze green

I978 Ioc blue green

I979 I2c green

I980 I2c yellow green

I98 I I2c bronze green

I982 I2c blue green

I983 20c green

I984 20c yellow green

I985 $20 \mathrm{c}$ bronze green

I $98620 \mathrm{c}$ blue green 



\section{.}




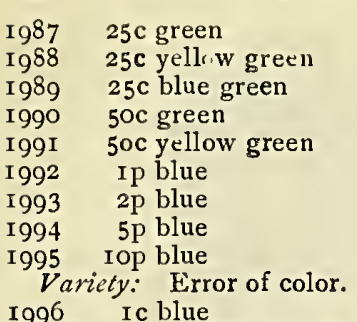

r9g6 Ic blue

This stamp was only discovered about a year ago, a nnmber of sheets having been sold by Mexican dealers; it is more than probable that they are only essays.

$3^{\circ}$ Thick opaque wove paper. Size $21 \frac{1}{2}$ $\times 261 / 2$ to $213 / 4 \times 263 / 4 \mathrm{~mm}$.

Perforated I2.

\begin{tabular}{|c|c|}
\hline I 997 & IC green \\
\hline & Ic yellow green \\
\hline I 999 & Ic bronze green \\
\hline & Ic blue green \\
\hline OI & $2 c$ green \\
\hline 02 & $2 c$ yellow green \\
\hline 03 & 2: bronze green \\
\hline 04 & $2 \mathrm{c}$ blue green \\
\hline 05 & $3 c$ green \\
\hline 00 & 3c yellow green \\
\hline & 3c bronze green \\
\hline oo & $\begin{array}{l}3 c \text { blue green } \\
4 c \text { green }\end{array}$ \\
\hline Io & 4c yellow green \\
\hline II & 4c bronze green \\
\hline & $4 \mathrm{c}$ blue green \\
\hline $\mathrm{bI} 3$ & $5 \mathrm{c}$ green \\
\hline & $5 \mathrm{c}$ yellow green \\
\hline & $5 \mathrm{c}$ bronze green \\
\hline & $\begin{array}{l}5 \mathrm{c} \text { blue gree } \\
6 \mathrm{c} \text { green }\end{array}$ \\
\hline 18 & 6c yellow green \\
\hline IO & $6 \mathrm{c}$ bronze green \\
\hline 20 & $6 \mathrm{c}$ blue green \\
\hline & IOc gre \\
\hline 022 & IOc yellow green \\
\hline & IOc bronze gree \\
\hline 024 & Ioc blue green \\
\hline & I $2 \mathrm{c}$ green \\
\hline 126 & I2c yellow green \\
\hline 227 & I2c bronze gree \\
\hline & I2c blue green \\
\hline & $20 c$ green \\
\hline & 20c yellow \\
\hline & $20 c$ blue green \\
\hline
\end{tabular}

The perforating of the stamps of this issue was done very irregularly, some stamps having absolutely no margins and others having very wide margins; we have seen some with margins measuring $3 \mathrm{~mm}$. on each side. This accounts for the great number of so-called unperforated stamps of this issue, as it would be very easy to manufacture them from the pelforated specimens with wide margins. Of course some sheets have escaped the perforating machine, and were thus sold, but we advise collectors to be suspicinus of unperforated stamps of this and following issues, unless in unsevered pairs or strips. The same can be said about part perforated stamps of this issue, as the margins of the shee's were generally not perforated, thus causing all the outside stamps to be unperforated.

District Names found on this Issue.

Cuernavaca 20x2; black surcharge

Chiapas

Etzatlan

Guanajuato

$14 \times 2$

"“

I $61 / 2 \times 13 / 4$

(Heitman's Handbuch)
$\mathrm{La} \mathrm{Paz}$

Maravatio

Mazatlan

Salıillo

T. cubaya

Soyaniquilpam

Tehuantepec

Texcoco

Zumpango

July Ist, I 885

Same type as preceding issue, but stamps printed in various colors on various papers. Size $211 / 2 \times 27$ to $22 \times 271 / 3$.

$I^{\circ}$ Horizontally laid paper, varying in thickness.

Perforated I2.

2033 Ic pale green

2034 2c carmine

2035 3c red brown

$2036 \quad 4 \mathrm{c}$ red

$2037 \quad 5 \mathrm{c}$ blue

2038 6c brown

2039 IOc yellow

2040 IOc orange

204I I2c black brown

$2042 \quad 25$ c greenish blue

$2^{\circ}$ Ordinary white wove paper varying in thickness.

Perforated 12.

$2043 \quad$ Ic pale green

$2044 \quad 2 c$ carmine

$2045 \quad 3 \mathrm{c}$ red brown

$2046 \quad 4 c$ red

$20475 \mathrm{c}$ blue

$2048 \quad 6 \mathrm{c}$ brown

2049 Ioc yellow

2050 IOc orange

205 I I2c black brown

$205225 \mathrm{c}$ greenish blue

What we have said in regard to the unperforated and part perforated stamps of the preceding issue applies also to the stamps of this is sue.

District Names Found on THIS Issue.

Acayucam $\quad$ I $81 / 2 \times 31 / 2$; black surcharge

Etzatlan

Sanmarcial

Tabasco $20 \times 2$

$16 \mathrm{I} / 2 \times 13 / 4-$ “"

Tehuantepec $25 \times 23 / 4$ in MS. 23x I 3/4; black surcharge

July Ist, I 886 .

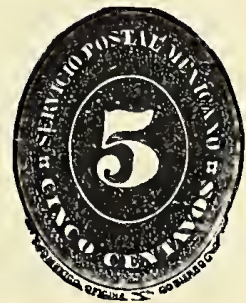

$\mathrm{I}^{\circ}$ Plain white wove paper varying in texture. Size $23 \times 281 / 2 \mathrm{~mm}$.

Perforated I2

2053 Ic blue green

2054 Ic yellow green

2055 2c carmine

$2056 \quad 3 \mathrm{c}$ lilac

$2057 \quad 4 \mathrm{c} l \mathrm{lac}$

$2058 \quad 5 \mathrm{c}$ blue

$20596 \mathrm{c}$ lilac

ec60 Ioc lilac 
206I I2c lilac

$206220 \mathrm{c}$ lilac

$206325 \mathrm{c}$ lilac

The only surcharges known on the stamps of this issue are:

Acayucam I $\quad$ I $1 / 2 \times 3 \frac{1}{2}$; black surcharge

Maravatio

Tehuantepec

I $81 / 2 \times 31 / 2 ;$ black surcharge
$\begin{aligned} & 161 / 2 \times 2 \\ & 20 \times 2\end{aligned}$

August, I 887.

Same type as preceding issue, engraved on plain or ruled white wove paper, varying in texture

I. Plain white wove paper.

Pin perforated 6 .

2064 Ic blue green

2065 2c carmine

$20665 \mathrm{c}$ blue

2067 Ioc brown lilac

II. White wove paper, ruled with blue lines either on face or reverse or on both sides; such as is customarily used for ledgers and other commercial books.

$I^{\circ}$ Perforated 12.

$\begin{array}{ll}2068 & \text { Ic blue green } \\ 2069 & \text { 2c carmine } \\ 2070 & 5 \mathrm{c} \text { blue } \\ 2^{\circ} & \text { Pin Perforated } 6 . \\ 207 \mathrm{I} & \text { Ic blue green } \\ 2072 & \text { 2c carmine } \\ 2073 & 5 \mathrm{c} \text { blue } \\ 2074 & \text { 10c brown lilac }\end{array}$

I 887-88.

Same type as preceding issues, but colors of stamps different.

I. Plain white wove paper.

$I^{\circ}$ Perforated I2.

2075 2c carmine

$2076 \quad 3 \mathrm{c}$ scarlet

2077 4c “

2078 6c “

2079 IOC “"

2080 20c “

$2^{\circ}$ Pin Perforated 6.

2082 2c carmine

2083 IOc scarlet

(August, 1887)

II. White wove paper, ruled with blue lines, on face or reverse, or on both sides.

I 0 Perforated $\mathbf{2}$.

2084 2c carmine

2085 3c scarlet

(August, 1887)

2086 IOC

$2087 \quad 20 \mathrm{c}$

$2^{\circ}$ Pin perforated 6

2088 2c carmine

2089 3c scarlet

2090 roc “"

209 I $20 \mathrm{C}$ “"

(August, I887)

October, 1887

(End 1888 )

(August, I887)

(August, I888)

We have never seen the $3 \mathrm{c}$ or $20 \mathrm{c}$ in either perforation.

\section{April, I 888 .}

Provisional issue, issued in the town of Colima; stamp of regular issue of $1886-88$ surcharged in black or blue "I centavo" as per illustration.

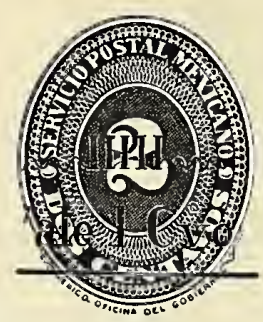

I. Plain white wove paper.

$I^{\circ}$ Perforated $\mathbf{2}$.

2092 Ic on $2 \mathrm{c}$ carmine, black surcharge

2093 Ic on $2 c$ carmine, blue surcharge

$2^{\circ}$ Pin perforated 6.

2094 Ic on 2c carmine, blue surcharge

II. White wove paper, ruled with blue lines.

$I^{\circ}$ Perforated I2.

2095 Ic on $2 c$ carmine, blue surcharge

2096 Ic on 2c " black

Varieties: Surcharge inverted.

2097 Ic on 2c carmine, blue surcharge

2098 Ic on 2c " " black "

$2^{\circ}$ Pin perforated 6.

2098a Ic on $2 c$ carmine, blue surcharge

The stamps with black surcharge are given on the authority of J. B. Moens.

The authenticity of this provisional stamp is denied by the authors of Heitman's Handbuch, who say that this stamp is an American swindle; that the stamp was originated by Sr. Escalante is probable, but that these stamps were sold at the Colima postoffice and prepaid mail matter not only in the interior but also to this country is undoubted; on the day that this stamp was said to have been issued we, ourselves received a letter from Dr. J. W. Bastow, the well known Mexican coin collector, prepaid with these stamps, and in his letter he calls attention to the fact of his using the stamps in question.

July, I89I.

Same type as regular issue of $1886-88$ engraved on various papers.

I. Paper watermarked CORREOS E. U.M. in every horizontal row of Io stamps, so that each stamp shows part of the watermark.

$\mathrm{I}^{\circ}$ White wove paper.

Perforated I2.

2099 Ic yellow green

$2100 \quad 2 c$ carmine

2IOI 3c vermilion

$2 \mathrm{IO} 2 \mathrm{c}$ "

$2 \mathrm{IO} 35 \mathrm{c}$ blue

$21046 \mathrm{c}$ vermilion

2105 IOC "

2106200 "

$210725 \mathrm{c}$

$2^{\circ}$ Vertically laid white paper.

2108 Ic green

2109 2c carmine

2 I IO $3 \mathrm{C}$ vermilion

2III 4c

2 II2 $5 \mathrm{c}$ blue

2 II3 IOc vermilion

II. Vertically laid white paper, unwatermarked.

Perforated I2. 

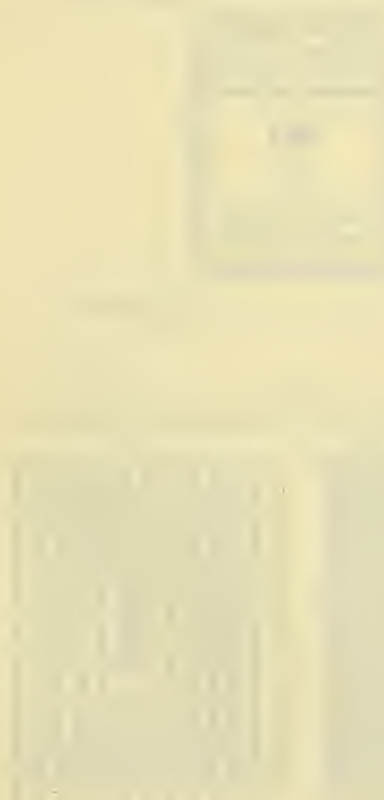

(1) - n 

1892.

I. Same type as preceding issue, engraved on white wove paper, watermarked CORREOS $E U M$ on every horizontal row of ro stamps. Perforated I2.

2 IIS IC blue green

2 II6 2c vermillion

$2 \operatorname{rr} 75 \mathrm{p}$ scarlet

II. Same type as issue of January, I887 (Head of Hidalgo), engraved on white wove paper, watermarked CORREOS E UM on every horizontal row of ro stamps.

Perforated 12.
2 Ir8 IOp "6

\section{$2 \operatorname{rrg} 5 \mathrm{p}$ blue green \\ End of 1892.}

Same type as preceding issue, (figure of value), engraved on white wove paper, unwatermarked.

Perforated $I 2$.

\begin{tabular}{|c|c|c|}
\hline $2 I 2 r$ & \multicolumn{2}{|c|}{$2 c$ vermilion } \\
\hline 2122 & $3 c$ & \\
\hline 2123 & $4 \mathrm{c}$ & " \\
\hline 2124 & $6 \mathrm{c}$ & “ \\
\hline 2125 & IOc & “ \\
\hline 21.26 & $20 \mathrm{C}$ & “" \\
\hline 127 & $25 \mathrm{C}$ & “ \\
\hline
\end{tabular}

UNPAID LETTER STAMPS.

Issued by the postmasters of various cities. *

Colàya.

I89r.

Typographed on white wove paper. Size r $81 / 2 \times 26 \mathrm{~mm}$.

Perforated $\mathrm{xr}$.

$250120 \mathrm{red}$

These stamps were also used by the postoffice of the City of Mexico.

$18 \mathrm{gr}$.

Typographed on bluish wove paper. Size $2 \mathrm{r} / 2 \times 25 \mathrm{~mm}$.

Perforated II.

2502 20c red

These stamps were also used by the Vera Cruz postoffice.

$189 \mathrm{r}$.

Typographed on bluish wove paper. Siz $2 \pi \mathrm{I} / 2 \times 26 \mathrm{~mm}$.

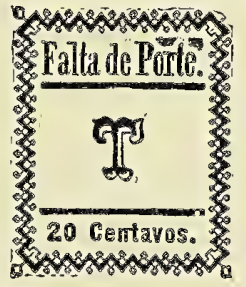

Perforated II.

2503200 red

1892 .

Typographed on white wove paper with various groundworks. Size $21 \% 30 \mathrm{~mm}$.

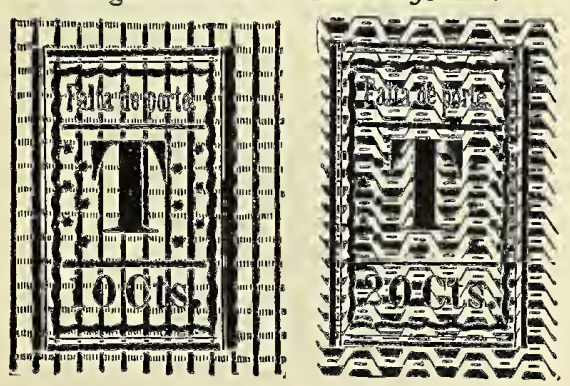

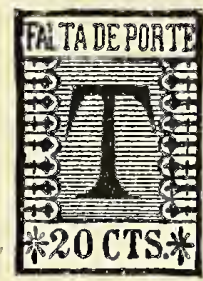

Irapuato.

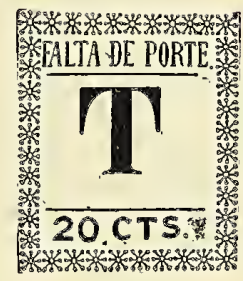

Mexico.

Rouletted.

2504 IOc black, green groundwork

$250520 c$ deep green, brown ground work

Typographed on white wove paper. Size $\mathrm{r} 8 \times 29 \mathrm{~mm}$.

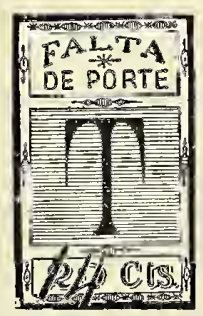

Perforated II.

$250620 \mathrm{c}$ mauve

2507 I4C mauve, black surcharge in MS

We are not positive that these stamps were issued by the postoffice of the City of Mexico.

*Note.-It has been a question whether these stamps were issued officially or whether they were created by speculativeiy inclined postmasters; h.owever, this has been settled by the letter of the Administrator of the Postoffice of Mexico, dated July 26 th, $\$ 892$, stating that their issue had been sanctioned by the Mexican Government. 
Typographed on white wove paper. Size $20 \times 28 \mathrm{~mm}$.

Perforated I $\mathbf{x}$.

2508 no value, mauve

$25098 \mathrm{c}$ green

25 IO $20 \mathrm{c}$ red

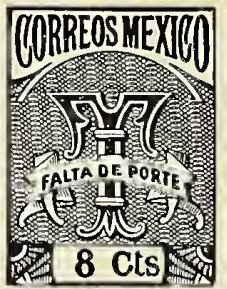

\section{Vera Cruz.}

I89I,

Typographed on white wove paper, groundwork of diagonally crossed lines. Size $21 \times 25 \mathrm{~mm}$.

Perforated II

$25 \mathrm{II} 20 \mathrm{C}$ red green groundwork

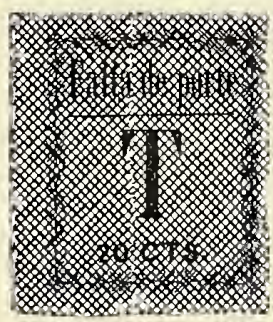

PORTE DE MAR.

Letters sent abroad via English or French packet steamers were charged double postage, i.e. three times more than inland postaye. This additional postage was paid by means of special stamps crealed for this purpose.

July, I875.

Lithographed on yellow wove paper, one plate for all the values, composed of 49 stamps in seven rows of 7 stamps each; the several values arianged on the plate as per following diagram. Size of stamp $28 \times 34 \mathrm{~mm}$.

$\begin{array}{rrrrrrr}\text { IO } & \text { IO } & 25 & 35 & 50 & 60 & 85 \\ \text { IO } & \text { 10 } & 25 & 35 & 50 & 75 & \text { 1OO } \\ \text { IO } & \text { IO } & 25 & 35 & 50 & 60 & 85 \\ \text { IO } & \text { IO } & 25 & 35 & 50 & 75 & 100 \\ \text { IO } & 10 & 25 & 35 & 50 & 60 & 85 \\ \text { 10 } & 10 & 25 & 35 & 50 & 75 & 100 \\ \text { O } & \text { IO } & 25 & 35 & 50 & 60 & 85\end{array}$

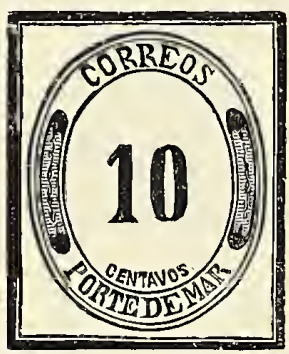

\footnotetext{
260 I roc black

$260225 \mathrm{C}$ “"

$2603 \quad 35$ cblack

$260450 \mathrm{c}$ “"

$260560 c$ “"

$260075 \mathrm{c}$ “"

$260785 \mathrm{c}$ “"

2008 I00c "
}

These stamps were never put into use, having been replaced by the following issue:
August, I 875 .

Lithographed on various papers; figure of value mea-uring $7 \mathrm{~mm}$, in height, and CENTAVOS measuring $71 / 2 \mathrm{~mm}$. in width. This issue consists of five plates of 56 stamp; in eight horizontal rows of seven stamps each. One plate for each of the 2, IO, I2 and 20 centavos values, and one plate for the other values, this last plate being composed as $\mathrm{per}$ following diagram:

$\begin{array}{rrrrrrr}25 & 35 & 50 & 60 & 60 & 85 & 85 \\ 25 & 35 & 50 & 75 & 75 & \text { 100 } & 100 \\ 25 & 35 & 50 & 50 & 60 & 85 & 85 \\ 25 & 35 & 50 & 75 & 75 & 85 & 100 \\ 25 & 35 & 50 & 60 & 60 & 85 & 85 \\ 25 & 35 & 50 & 75 & 75 & 85 & 100 \\ 25 & 35 & 50 & 60 & 60 & 85 & 85 \\ 25 & 35 & 50 & 75 & 75 & 85 & 100\end{array}$

The original plate used to make all the others was of the ro centavos value; the other plates were made by removing the figures ' $\mathrm{I}$ ' and ' $\mathrm{O}$ ' and replacing them by others, except for the ' 12 ' where the ' $O$ ' only was removed and replaced by ' 2 ' and the ' 20 ' where the 'I' was removed and also replaced by ' 2 '. These changes can easily be detected by a thin frame surrounding the inserted figures. In many instances this frame is very faint and sometimes only a small part of it can be seen. Size of stamp $251 / 2 \times 31 / 2 \mathrm{~mm}$

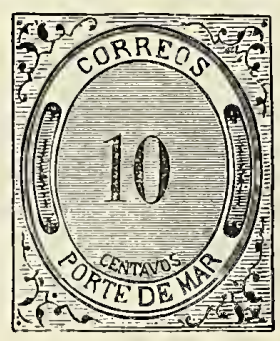

I, Without surcharge of name of postal district. 

I Yellowish white wove paper.

\begin{tabular}{|c|c|c|}
\hline 2609 & \multicolumn{2}{|c|}{ 2c black } \\
\hline 2610 & IOC & $"$ \\
\hline $26 I I$ & $I 2 C$ & "، \\
\hline $26 I 2$ & $20 \mathrm{c}$ & " \\
\hline $26 I_{3}$ & $25 \mathrm{C}$ & 66 \\
\hline $26 r 4$ & $35 \mathrm{c}$ & " \\
\hline $26 I_{5}$ & $50 \mathrm{C}$ & si \\
\hline $26 I 6$ & $60 c$ & 8 \\
\hline 2617 & $75 \mathrm{c}$ & 66 \\
\hline 2618 & $85 c$ & “ \\
\hline 2619 & roOc & " \\
\hline $2^{\circ}$ & Blui & h white wove paper. \\
\hline 520 & $2 \mathrm{C}$ & olack \\
\hline & IOC & 6 \\
\hline & I $2 \mathrm{C}$ & 6 \\
\hline & $20 \mathrm{C}$ & 66 \\
\hline 24 & $25 \mathrm{c}$ & 6" \\
\hline 2625 & $35 \mathrm{c}$ & " \\
\hline 2626 & $50 \mathrm{c}$ & $\cdot$. \\
\hline 2627 & $60 c$ & " \\
\hline 2628 & $75 \mathrm{c}$ & " \\
\hline & $85 \mathrm{c}$ & " \\
\hline 2630 & IoOc & " \\
\hline
\end{tabular}

Variety: Surcharged on reverse 5480 263I $60 \mathrm{c}$ black

II. With surcharge of name of postal district.

$I^{\circ}$ Yellowish white wove paper.

\begin{tabular}{|c|c|c|}
\hline & c & 190 \\
\hline 2633 & roc & \\
\hline 26 & $\mathrm{I} 2 \mathrm{c}$ & \\
\hline & $20 \mathrm{c}$ & \\
\hline & $25 \mathrm{C}$ & "4 \\
\hline 263 & $35 \mathrm{c}$ & \\
\hline & $50 \mathrm{c}$ & \\
\hline & $60 c$ & \\
\hline & $75 \mathrm{c}$ & \\
\hline & $85 \mathrm{c}$ & \\
\hline & $100 \mathrm{C}$ & \\
\hline
\end{tabular}

$2^{\circ}$ Bluish white wove paper.

2643 2c black

2644 IOC ".

2645 I2C "

2646200 66

$264725 \mathrm{C}$ "

$2648 \quad 35 \mathrm{C}$

$264950 \mathrm{C}$ "

$265060 \mathrm{c}$ "

$265 \mathrm{I} \quad 75 \mathrm{C}$

$2652 \quad 85 \mathrm{c}$ "

2653 IOOC "

NAMES OF SURChaRges Found ON STAMPS OF THIS ISSUE.

Apam $91 / 2 \times 2 ;$ blue surcharge

Chiapas $9 \frac{\pi}{4} \times 2$ to $2 \pi / 2$; blue surcharge

Guadalajara r $4 \times 1 / 4$; blue surcharge

Queretaro

Saltillo

Tacubaya

Tampico

Tula

Tula de $\mathrm{T}$

Vera Cruz

Zacatecas

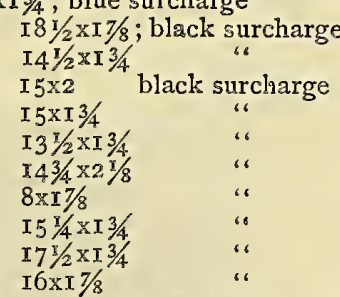

Mr. J. H. Anheisser in his article on Porte de Mar stamps, in the Stamp News Annual for 1892 , describes another series of this type on yellowish or bluish while wove paper, in which the figures of value of all the stamps are an integral portion of the design and have not been inserted. To the value already known he adds the $5 \mathrm{c}$ stamp; these stamps, however, are counterfeits. Comparison of the stamps of the first series with those given by Mr. Anheisser will easily prove our assertion; in the counterfeits the letters of the word "CENTAVOS" are higher than in the genuine; the same with the letters of the PORTE DE MAR, in which the R of MAR measures $2 \mathrm{~mm}$. in the counterfeits and $\mathrm{r} 3 / 4 \mathrm{~mm}$. in the genuine; the ornament in the upper left corner is also different in the counterfeits; in the genuine the S of CENTAVOS, although smaller than the other letters of this word, is on a line with the other letters, while in the counterfeits it extends beyond the line at the top. We have seen a whole set of these counterfeits surcharged OFFICIAL and CORREOS in varjous types and colors.

B. Same type, lithographed on various papers, the figures of value are larger $(8 \mathrm{~mm}$. in height) than in the preceding issue, and the word CENTAVos measures $9 \mathrm{x} / 2 \mathrm{~mm}$. in width. There is one distinct plate for each value, each plate composed of 56 stamps in 8 horizontal rows of 7 stamps each. Size of stamp $25 \frac{1}{2} \times 32 \mathrm{~mm}$.

I. Without surcharge of name of postal district.

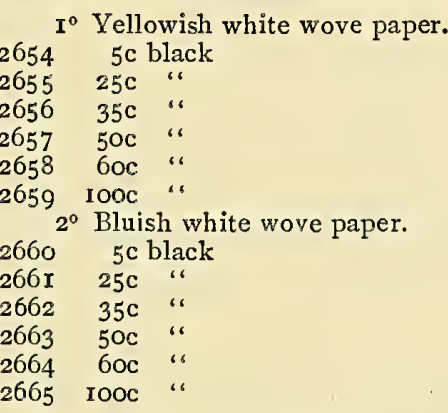

II. With surcharge of name of postal district.
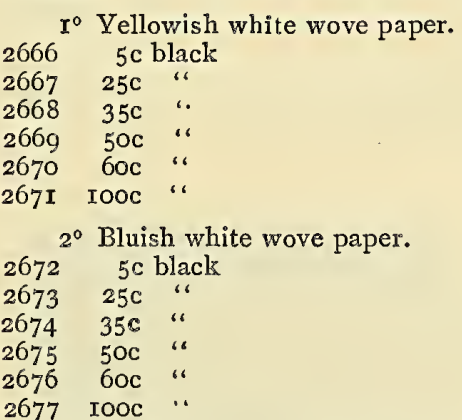

Surcharges of names of Postal Districts FOUND ON THE STAMPS OF THIS ISSUE.

\begin{tabular}{|c|c|c|}
\hline Queretaro & \multicolumn{2}{|c|}{ I4 1/2x I 3/4; black surcharge } \\
\hline Vera Cruz & I $71 / 2 \times 13$ & " \\
\hline "، & I $7 \times 2$ & “ \\
\hline “" & $18 \times 2$ & " \\
\hline Zacatecas & I $6 \times I 7 / 8$ & "6 \\
\hline
\end{tabular}

January Ist, I879.

Lithographed on white wove paper, without name of postal di-trict. Each sheet is watermarked "Administracion General de Correos Mexico" in large double lined letters. Size of stamps I $9 \times 25$. 


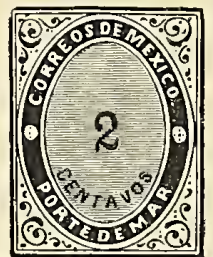

2678 2c brown

2679 5c yellow

2680 Ioc red

268 I 25c blue

2682 5Oc green
2683 IOoc violet

Pin perforated (unoffically).

2684 2c brown

$2685 \quad 5$ c yellow

2686 IOc red

$268725 \mathrm{c}$ blue

2688 50c green

2689 IOOC violet

In some catalogues a series of stamps is given of similar type with small star above figure of value printed in the same colors as the stamps. These, however, are counterfeits or humbugs, a fact which is now beyond dispute.

OFFICIAL STAMPS.

I $884-85$.

Engraved on various papers. Size $2 I \frac{1}{2} \mathrm{x}$ $27 \mathrm{~mm}$.

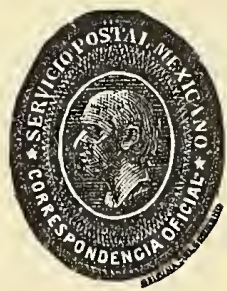

I White wove paper.

Perforated I2.

2800 bright red (May, 1884)

$2^{\circ}$ Wide horizontally laid paper.

Perforated $\mathbf{I} 2$.

2801 bright red (October, 1884)

$3^{\circ}$ Narrow horizontally laid paper.

Perforated I 2 .

2802 bright red (January, I885)

July, I 887.

Same type as preceding issue, engraved on various papers.

I. White wove paper.

I 885 .

OFFICIALLY SEALED STAMPS.

Lithographed on white wove paper. Size $431 / 2 \times 26 \mathrm{~mm}$.

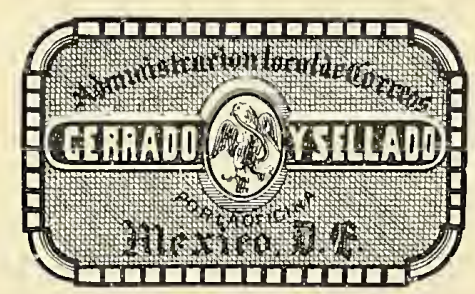

$I^{\circ}$ With Mexico, D. F.

2900 brown

$2^{\circ}$ With Mexico, D. F, erased.

290I brown

I888.

Lithographed on white wove paper. Size $43 \mathrm{r} / 2 \times 26 \mathrm{~mm}$.
I $^{\circ}$ Perforated I 2.
2803 brown
$2^{\circ}$ Pin perforated 6.
2804 brown

II. Wide horizontally laid paper.

Perforated I2.

2805 brown

I889.

Same type as preceding issue, engraved on various papers.

$I^{\circ}$ White wove paper.

Perforated I2.

2806 vermilion

$2^{\circ}$ Narrow horizontally laid paper.

Perforated I2.

2807 vermilion

I893.

Same type as preceding issues, engraved on white wove paper.

Perforated I2.

2808 green

We have seen some adhesives of the 1884 issue surcharged in violet "Conductor de Correspondencia," but so far we have not been able to get any evidence to show that these stamps were used as officials, for it is possible that this surcharge is merely a cancellation.

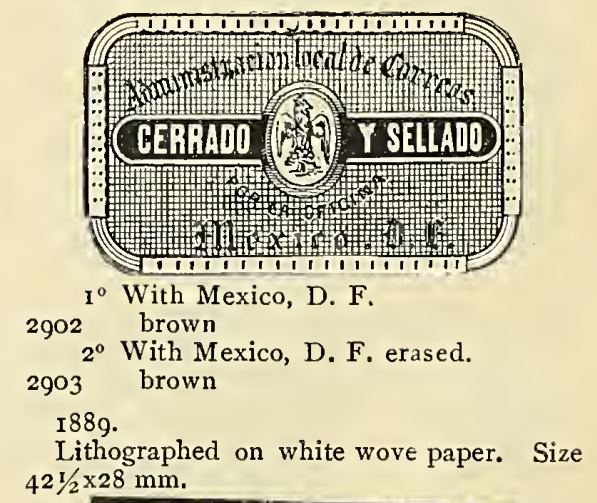
$42 \mathrm{l} / 2 \times 28 \mathrm{~mm}$.

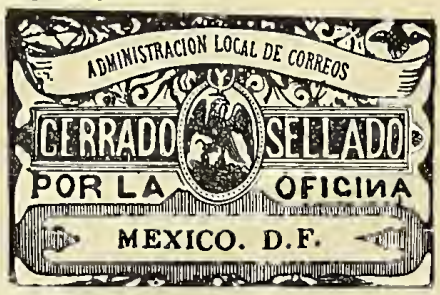

I With Mexico, D. F.

2904 brown

$2^{\circ}$ With Mexico, D. F. erased.

2905 brown 


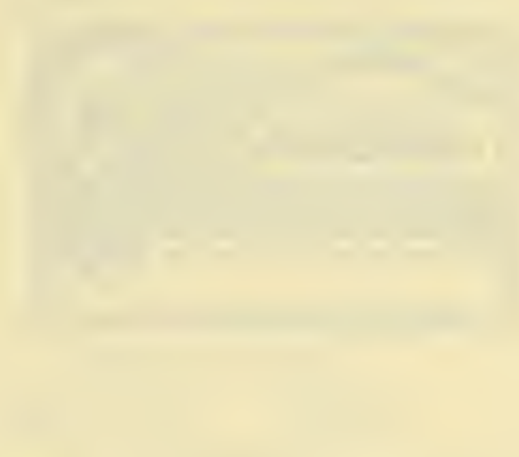

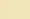



I891.

Same type as preceding issue.

$I^{0} \cdot$ With Mexico, D. F.

2906 mauve

$2^{0}$ With Mexico, D. F. erased.

2907 mauve

I892.

Lithographed on white wove paper. Size $49 \times 33 \mathrm{~mm}$.

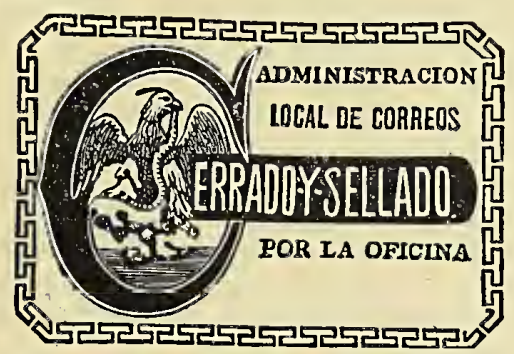

I $^{\circ}$ With Mexico, D, F.

2908 mauve

$2^{\circ}$ Without Mexico, D. F.

2909 red brown

May 5 th, I 874 .

Stamp embossed in upper right corner; white paper, laid diagonally.

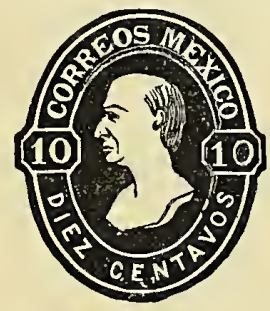

$r^{\circ}$ Without surcharge.

Size $140 \times 83 \mathrm{~mm}$.

3001 roc yellow green

3002 IOc green

3003 roc dark green .

$300425 \mathrm{c}$ dark blue

$2^{\circ}$ Surcharged with number of postal district and abbreviated date in black in upper left corner of envelope.
1892-93.

Lithographed on white wove paper. Size $47 \mathrm{I} / 2 \times 30 \mathrm{~mm}$.

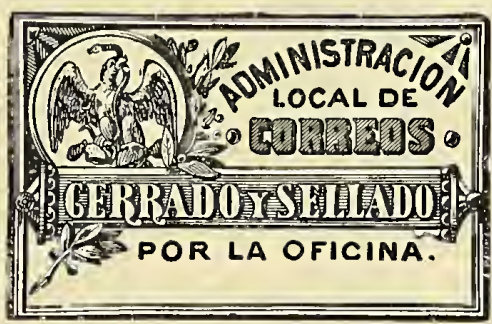

$291 \mathrm{I}^{\circ}$ Without inscription.

291 brown (I893)

$2^{\circ}$ With inscription.

2912 rose Chihuahua, in violet $35 \times 5$

2913 " “" " " $25 \times 21 / 2$

2914

" in blue

I 894 .

Same type as preceding issue, but reengraved; typographed on white wove paper.

Perforated $13 \frac{1}{2}$.

29I5 brown

ENVELOPES.

Size $140 \times 83 \mathrm{~mm}$.

3005 IOc yellow green

3006 Ioc green

3007 Ioc dark green

$300825 \mathrm{C}$ dark blue

Variety: Stamp inside of envelope, with number and date on back of stamp.

$300925 \mathrm{c}$ dark blue

$3^{\circ}$ Surcharged with number of postal distr.ct and abbreviated date in upper left corner in black, and wilh name of postal district in black or in color across, above or below the stamp.

Size $140 \times 83 \mathrm{~mm}$.

3oro roc yellow green

30 II IOC green

3012 roc dark green

$301325 \mathrm{c}$ dark blue

Stamp of same type as preceding issue, embossed on white paper, laid diagonally. Watermarked eagle and Admon. Gral de Correos above and Mexico below. Name of maker, "Gonthier, Dreyfus \& Cie., Paris" on left flap.

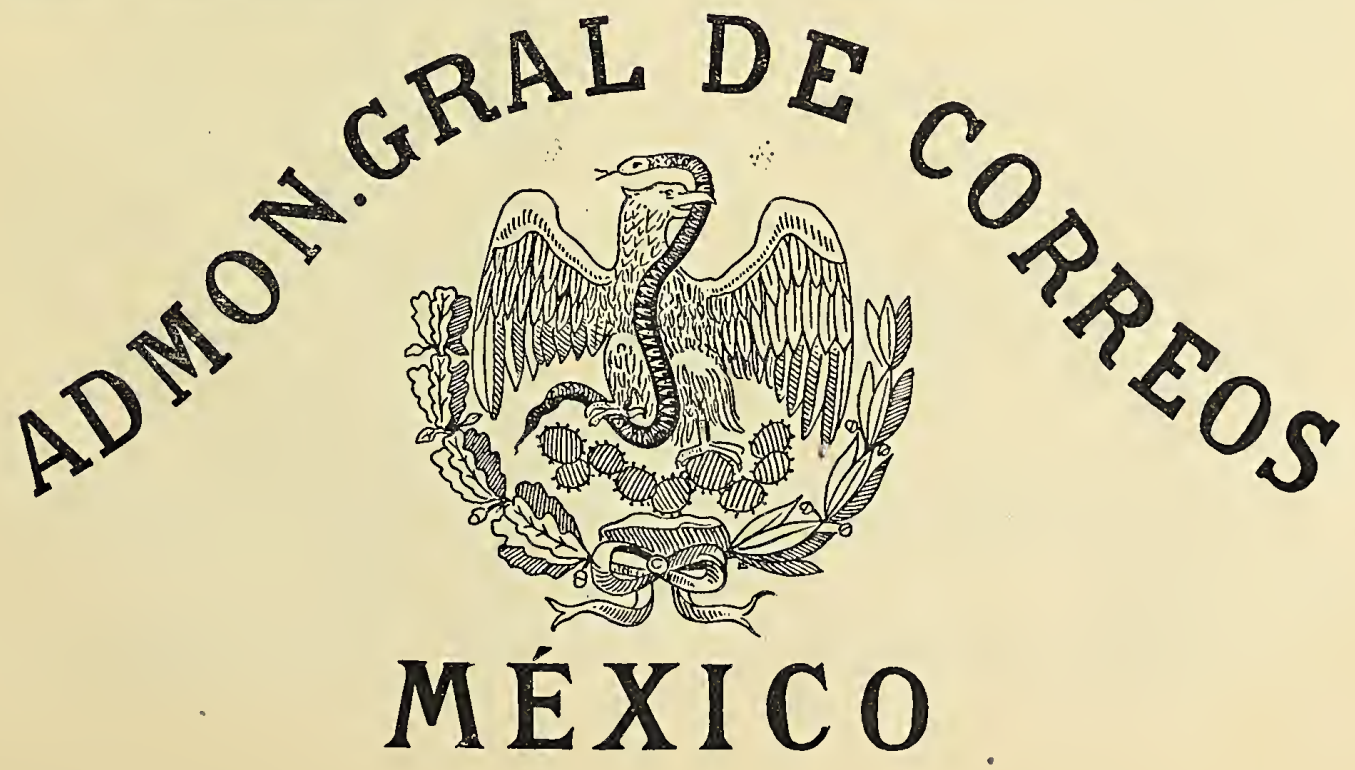


$I^{\circ}$ Without surcharge.

Size $140 \times 83 \mathrm{~mm}$

3014 IOc green

3015 IOc pale green

3०I6 Ioc deep green

$301725 \mathrm{c}$ blue

$3018 \quad 25 \mathrm{c}$ pale blue

30I9 25c dark blue

$3020 \quad 25 \mathrm{c}$ ultramarine

$2^{\circ}$ Surcharged with number of postal

district and abbreviated date in black.

Size $140 \times 83 \mathrm{~mm}$.

3021 IOc green

3022 IOc pale green

3023 Ioc deep green

$3023 \mathrm{a} 25 \mathrm{c}$ blue

$302425 \mathrm{c}$ pale blue

$3025 \quad 25 \mathrm{c}$ dark blue

$3026 \quad 25 \mathrm{c}$ ultramarine

$3^{\circ}$ Surcharged with number and abbreviated date in black, and name of postal district in black or color.

Size $140 \times 83 \mathrm{~mm}$.

3027 IOc green

3028 Ioc pale green

3029 Ioc deep green

$3030 \quad 25 \mathrm{c}$ blue

303 I 25c pale blue

$3032 \quad 25 \mathrm{c}$ deep blue

$303325 \mathrm{c}$ ultramarine

a. Double impression of stamp, one without color.

$3034 \quad 25 \mathrm{c}$ pale blue

b. Paper laid vertically.

3035 Ioc green

$3036 \quad 25 \mathrm{c}$ blue

$3037 \quad 25$ c pale blue

Stamp of same type as preceding issues, embossed on white paper laid diagonally, without watermark and without name of maker.

$I^{\circ}$ Without surcharge.

Size $140 \times 83 \mathrm{~mm}$.

$3038 \quad 4 c$ pale red

$2^{\circ}$ Surcharged with number of postal

district and abbreviated date, in black.

Size $140 \times 83 \mathrm{~mm}$.

$3039 \quad 4 c$ pale red

$3^{\circ}$ Surcharged with number and abbreviated date, in black, and name of postal district, in black or color.

Size $140 \times 83 \mathrm{~mm}$.

$3040 \quad 4 \mathrm{c}$ pale red

1 arieties:

a. Stamp embossed in centre of envelope. 304I 4C pale red

b. Double impression of stamp.

$3042 \quad 4 \times 4 c$ pale red

March, I880.

Same as preceding issue, but paper watermarked as issue of January, I 879 , and name of maker "Gonthier, Dreyfus \& Cie., Paris,' on left flap.

$I^{\circ}$ Without surcharge.

Size $140 \times 83 \mathrm{~mm}$.

3043 4c pale red

$3044 \quad 4 \mathrm{c}$ orange red

$2^{\circ}$ Surcharged with number of postal

district and abbreviated date in black.

Size $\mathrm{I}_{40 \times} 83 \mathrm{~mm}$.

$3045 \quad 4 \mathrm{c}$ pale red

$3046 \quad 4 \mathrm{c}$ orange red

$3^{\circ}$ Surcharged with number and abbre- viated date in black, and name of postal district in black or color.

Size $140 \times 83 \mathrm{~mm}$.

$3047 \quad 4 c$ pale red

3048 4c orange red

Varieties:

a, Double impression of stamp.

$3049 \quad 4 \times 4 c$ pale red

b. Part of second stamp impressed without color below first stamp.

$3050 \quad 4 c$ pale red

c. Vertically laid paper.

$305 \mathrm{I} \quad 4 \mathrm{c}$ pale red

30524 4c orange red

d. Stamp impressed without color. $3053 \quad 4 \mathrm{C}$

April, I882.

Envelopes of the issue of I874 surcharged HABILITADO (3IX3 $3 / 4 \mathrm{~mm}$.), below stamp. $I^{\circ}$ Surcharged with number of postal district and abbreviated date. White laid paper.

Size $140 \times 83 \mathrm{~mm}$.

3054 IOc green, green surcharge

3055 Ioc yellow green "
3056 25c dark blue, blue surcharge

$2^{\circ}$ Surcharged with number and name of postal district and abbreviated date. White laid paper.

Size $140 \times 83 \mathrm{~mm}$.

3057 IOc green, green surcharge

$305925 \mathrm{c}$ dark blue, blue surcharge

Variety: Habilitado surcharged four times.

$306025 \mathrm{c}$ dark blue, blue surcharge

End I882.

I. Stamp of same type as preceding issue embossed in upper right corner on diagonally laid white pafer.

Size $140 \times 83 \mathrm{~mm}$

$306 \mathrm{I} \quad 5 \mathrm{c}$ brown

3062 5c red brown

These envelopes are without surcharge as they never went into actual use.

II. Same as "I." with addition of stamp in upper left corner.

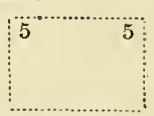

$I^{\circ}$ Without surcharge.

Size $140 \times 83 \mathrm{~mm}$.

$3063 \quad 5 \mathrm{c}$ brown $\times 5 \mathrm{c}$ brown

$3064 \quad 5 \mathrm{c}$ brown $\times 5 \mathrm{c}$ red brown

$3065 \quad 5 \mathrm{c}$ red brown $\mathrm{x} 5 \mathrm{c}$ red brown

$3066 \quad 5 \mathrm{c}$ red brown $\times 5 \mathrm{c}$ brown

$30675 \mathrm{c}$ red brown $\mathrm{x} 5 \mathrm{c}$ rose

$3068 \quad 5 \mathrm{c}$ brown $\mathrm{x} 5 \mathrm{c}$ rose

$3069 \quad 5 \mathrm{c}$ red brown $\mathrm{x}$ Ioc green

$2^{\circ}$ Surcharged with number of postal district and abbreviated date.

Size $140 \times 83 \mathrm{~mm}$.

$3070 \quad 5 \mathrm{c}$ brown $\mathrm{x} 5 \mathrm{c}$ brown

$307 \mathrm{I} 5 \mathrm{c}$ brown $\mathrm{x} 5 \mathrm{c}$ red brown

$3072 \quad 5 \mathrm{c}$ red brown $\times 5 \mathrm{c}$ brown

$30735 \mathrm{c}$ red brown $\times 5 \mathrm{c}$ red brown

$3074 \quad 5 \mathrm{c}$ red brown $\mathrm{x} 5 \mathrm{c}$ rose

$3075 \quad 5 \mathrm{c}$ brown $\mathrm{x} 5 \mathrm{c}$ rose

$30765 \mathrm{c}$ red brown $\mathrm{x}$ IOc green

$3^{\circ}$ Surcharged with number and name

of postal district and abbreviated date.

Size $140 \times 83 \mathrm{~mm}$.

$3077 \quad 5 \mathrm{c}$ brown $\mathrm{x} 5 \mathrm{c}$ brown

$3078 \quad 5 \mathrm{c}$ brown $\mathrm{x} 5 \mathrm{c}$ red brown

$3079 \quad 5 \mathrm{c}$ red brown $\times 5 \mathrm{c}$ brown 


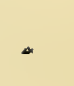


$3080 \quad 5 \mathrm{c}$ red brown $\times 5 \mathrm{c}$ redbrown

$308 \mathrm{I} \quad 5 \mathrm{c}$ red brown $\mathrm{x} 5 \mathrm{c}$ rose

$3082 \quad 5 \mathrm{c}$ brown $\mathrm{x} 5 \mathrm{c}$ rose

$3083.5 \mathrm{c}$ red brown $\mathrm{x}$ IOc green

Varieties.

a. The second stamp in lower right corner is inverted.

$3084 \quad 5 \mathrm{c}$ red brown $\mathrm{x} 5 \mathrm{c}$ brown

$\mathrm{b}$, The second stamp is printed in green, and is in the lower left corner.

$3085 \quad 5 \mathrm{c}$ brown $\times 5 \mathrm{c}$ yellow green

$30865 \mathrm{c}$ brown $\mathrm{x} 5 \mathrm{c}$ dark green

c. The second stamp is printed without color in lower left corner.

$3087 \quad 5 \mathrm{c}$ brown $\mathrm{x}$ Ioc

Varieties b and c exist only without surcharge.

III. Same as "II." with the addition of a third stamp in lower left corner.

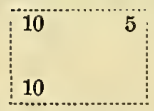

I ${ }^{\circ}$ Without surcharge.

Size $\mathrm{r} 40 \times 83 \mathrm{~mm}$.

3088 5c brown $\mathrm{x}$ IOxIOc yellow green

$3089 \quad 5 \mathrm{c}$ red brown $\mathrm{x}$ Ioxioc dark green

$3090 \quad 5 \mathrm{c}$ brown $\mathrm{x}$ roxroc blue green

309I 5c purple brown $\mathrm{x}$ IOxioc dark green

$2^{\circ}$ Surcharged with number of postal district and abbreviated date.

Size I40x83 mm.

$30925 \mathrm{c}$ brown $\mathrm{x}$ IOxIOc yellow green

$30935 \mathrm{c}$ red brown $\mathrm{x}$ IOxIOc dark green

$30945 \mathrm{c}$ brown $\mathrm{x}$ Ioxioc blue green

3095 5c purple brown $\mathrm{x}$ IOxIOc dark green

$3^{\circ}$ Surcharged with number and name of postal district and abbreviated date.

Size $140 \times 83 \mathrm{~mm}$

$3096 \quad 5 \mathrm{c}$ brown $\mathrm{x}$ roxroc yellow green

$30975 \mathrm{c}$ red brown $\mathrm{x}$ IOxIOc dark green

$3098 \quad 5 \mathrm{c}$ brown $\mathrm{x}$ IOxIOc blue green

$30995 \mathrm{c}$ purple brown $\mathrm{x}$ IOx IOc dark

Varieties: green

a. The second and third stamps are printed in green.

3 IOO $5 \mathrm{c}$ brown $\times 5 \times 5 \mathrm{c}$ green

3IOI $5 \mathrm{c}$ red brown $\mathrm{x} 5 \times 5 \mathrm{c}$ yellow green

These exist only without surcharge.

b. With additional stamp embossed without color.

$3 \mathrm{ro} 25 \mathrm{c}$ red brown $\times 5 \times 5 \mathrm{c}$ green $\mathrm{x} 5 \mathrm{c}$

c. The second roc stamp is inverted in right lower corner.

3 ro3 5c brown $x$ IoxIoc green

d. Both the roc stamps are inverted in low e corners.

3 ro 4 5c brown x IOxIOc green

e. The second roc stamp is leaning towards the lelt lower corner.

3 ro5 5c brown $x$ rox IOc green

f. The second IOc stamp slants towards the center of envelope.

3 IO6 5c brown $x$ roxIoc green

g. Both roc stamps are placed sideways in lower left corners.

3IO7 5c brown x IOxIOc green

IV. Same as III. with addition of a fourth stamp in lower right hand corner.

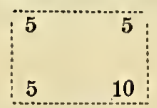

$I^{0}$ Without surcharge.
Size $140 \times 83 \mathrm{~mm}$.

3 Io8 $5 \mathrm{c}$ brown $\mathrm{x} 5 \times 5 \mathrm{c}$ green $\mathrm{x}$ Ioc yellow green

$3 \operatorname{Iog} 5 \mathrm{c}$ brown $\mathrm{x} 5 \times 5 \mathrm{c}$ dark green $\mathrm{x}$ IOc green

$2^{\circ}$ Surcharged with number of postal district and abbreviated date.

Size $140 \times 83 \mathrm{~mm}$.

3Iro $5 \mathrm{c}$ brown $\mathrm{x} 5 \times 5 \mathrm{c}$ green $\mathrm{x}$ roc yellow green

3III $5 \mathrm{c}$ brown $\mathrm{x} 5 \times 5 \mathrm{c}$ dark green $\mathrm{x}$ IOC

Varieties green

a. The roc stamp is in centre of lower part of envelope.

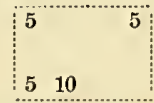

3 Ix 5 c brown $\times 5 \times 5 c$ green $x$ Ioc yellow green

b. Part of the roc stamp embossed over the $5 \mathrm{c}$ brown.

3 II 3 5 brown $\times 5 \times 5 c$ green $x$ roc yellow I883.

Stamp of same type as preceding issue, embossed on various papers.

I. Stamp in upper right corner.

A. Yellow wove paper.

$I^{\circ}$ Without surcharge.

Size $138 \times 78 \mathrm{~mm}$.

$3 \mathrm{II}_{4} 25 \mathrm{c}$ blue

3 II 5 25 dark blue

Size $148 \times 84 \mathrm{~mm}$.

$3 \mathrm{rr} 625 \mathrm{c}$ blue

3 II $75 \mathrm{c}$ dark blue

$2^{\circ}$ Surcharged with number of postal dis

trict and abbreviated date.

Size $\pi 38 \times 78 \mathrm{~mm}$,

3 II8 25c blue

3II9 $25 \mathrm{c}$ dark blue

Size $\mathrm{r} 48 \times 84 \mathrm{~mm}$.

$3 \mathrm{I} 2 \mathrm{O} \quad 25 \mathrm{c}$ blue

3 I2I 25c dark blue

$3^{\circ}$ Surcharged with number and name of Postal district and abbreviated date.

Size $\mathrm{I}_{3} 8 \times 78 \mathrm{~mm}$.

3 I22 $25 \mathrm{c}$ blue

3 r23 25c dark blue

Size $148 \times 84 \mathrm{~mm}$.

3 I $2425 \mathrm{c}$ blue

3r25 25c dark blue

B. White laid paper.

I ${ }^{\circ}$ Without surcharge.

Size $150 \times 82 \mathrm{~mm}$.

$3126 \quad 4 c$ orange red

Size $\mathrm{r} 53 \times 84 \mathrm{~mm}$.

3127 4c orange red

3128 IOc green

3 r29 25c blue

Size $149 \times 8 \mathrm{rmm}$

$3130 \quad 25 \mathrm{c}$ blue

$2^{\circ}$ Surcharged with number of postal

district and abbreviated date.

Size I $50 \times 82 \mathrm{~mm}$.

3I3I 4c orange red

Size $149 \times 81 \mathrm{~mm}$.

$3132 \quad 25 \mathrm{c}$ blue

Size I $53 \times 84 \mathrm{~mm}$.

3I 33 4c orange red

3 T34 IOC green

3I35. 25c blue

Varieties:

a. Vertically laid paper. 
$3136 \quad 4 \mathrm{c}$ orange red

3137 roc green

3I28 25c ultramarine

b. Horizontally laid paper.

3I 39 Ioc yellow green

C. White wove paper.

$I^{\circ}$ Without surcharge.

Size $153 \times 84 \mathrm{~mm}$.

$3 \mathrm{I} 4 \mathrm{O} 25 \mathrm{c}$ blue

$2^{\circ}$ Surcharged with number of postal

district and abbreviated date.

Size $153 \times 84 \mathrm{~mm}$.

3I 4I $25 \mathrm{c}$ blue

$3^{\circ}$ Surcharged with number and name of postal district and abbreviated date.

Size $153 \times 84 \mathrm{~mm}$.

$314225 \mathrm{c}$ blue

D. Straw laid paper.

$I^{\circ}$ Without surcharge.

Size $153 \times 84 \mathrm{~mm}$.

3I43 25c blue pointed flap

$3 \mathrm{I} 4425 \mathrm{c}$ blue rounded flap

$2^{\circ}$ Surcharged with number of postal

district and abbreviated date.

Size $153 \times 84 \mathrm{~mm}$.

$354525 \mathrm{c}$ blue pointed flap

$3 \mathrm{I} 46$ 25c blue rounded flap

$3^{\circ}$ Surcharged with number and name of postal district and abbreviated date.

Size $153 \times 84 \mathrm{~mm}$.

3 $44725 \mathrm{c}$ blue pointed flap

$3 \mathrm{I} 48$ 25 $\mathrm{C}$ blue rounded flap

E. Manila paper.

$I^{\circ}$ Without surcharge.

Size $150 \times 85 \mathrm{~mm}$.

$3149 \quad 25 \mathrm{C}$ blue

$2^{\circ}$ Surcharged with number of postal dis $_{3}{ }^{3}$ trict and abbreviated date.

Issue May $5^{\text {th }}, 1874$.

\begin{tabular}{|c|c|c|}
\hline Value. & Number and Date. & District Name. \\
\hline IOC & I 74 & Mexico (in half circle) \\
\hline IOC & 5074 & Vera Cruz \\
\hline IOc & 5074 & Vera Cruz \\
\hline $25 \mathrm{c}$ & 174 & Mexico (In half circle) \\
\hline $25 \mathrm{c}$ & 5074 & Vera Cruz \\
\hline $25 \mathrm{c}$ & 5874 & Tuxpan \\
\hline \multicolumn{3}{|c|}{ Issue of 1878 . } \\
\hline IOc & I 8 I & Zamora \\
\hline IOC & $48 \mathrm{I}$ & Hermosillo \\
\hline IOC & $78 \mathrm{I}$ & Tula de T. \\
\hline Ioc & II 82 & (Texcoco) \\
\hline IOC & I282 & (Tepic) \\
\hline IOC & $\times 580$ & (Tacubaya) \\
\hline IOC & 1879 & Saltillo \\
\hline IOC & 2181 & Pachuca \\
\hline IOC & 2779 & Mazatlan \\
\hline IOC & 3479 & Parral \\
\hline IOC & $398 \mathrm{I}$ & Chihuahua \\
\hline IOC & $468 I$ & Cuantitlan \\
\hline IOC & $498 I$ & Colima \\
\hline IOC & 5479 & Mexico (in half circle) \\
\hline IOC & 5480 & “ \\
\hline $25 \mathrm{c}$ & 180 & Zamora \\
\hline $25 \mathrm{c}$ & I8I & “ \\
\hline $25 \mathrm{c}$ & 280 & Zacatecas \\
\hline $25 \mathrm{c}$ & 379 & Vera Cruz \\
\hline $25 \mathrm{c}$ & $48 \mathrm{I}$ & Hermosillo \\
\hline $25 \mathrm{c}$ & $68 I$ & Tula de $\mathrm{T}$. \\
\hline $25 \mathrm{c}$ & 878 & Tuxpan \\
\hline $25 \mathrm{c}$ & 882 & \\
\hline $25 \mathrm{c}$ & 980 & Toluca \\
\hline
\end{tabular}

Size $150 \times 85 \mathrm{~mm}$.

$3150 \quad 25 \mathrm{c}$ blue

$3^{\circ}$ Surcharged with number and name of postal district and abbreviated date.

Size $150 \times 85 \mathrm{~mm}$.

3I5I 25c blue

II. Stamp in upper left corner.

A. White wove paper.

$\mathrm{I}^{\circ}$ Without surcharge.

Size $150 x 85 \mathrm{~mm}$.

$3152 \quad 25 \mathrm{c}$ blue

$2^{\circ}$ Surcharged with number of postal district and abbreviated date.

Size $150 \times 85 \mathrm{~mm}$.

$3 \mathrm{I} 5325 \mathrm{c}$ blue

$3^{\circ}$ Surcharged with number and name of postal district and abbreviated date.

Size $150 \times 85 \mathrm{~mm}$.

$3 \mathrm{I} 54 \quad 25 \mathrm{c}$ blue

B. White laid paper.

$I^{\circ}$ Without surcharge.

Siz $150 \times 85 \mathrm{~mm}$.

3I $5525 \mathrm{c}$ blue

$2^{\circ}$ Surcharged with number of postal district and abbreviated date.

Size $150 \times 85 \mathrm{~mm}$.

$315625 \mathrm{c}$ blue

$3^{\circ}$ Surcharged with number and name of postal district and date.

Size $150 x 85 \mathrm{~mm}$.

$3157 \quad 25 \mathrm{c}$ blue

Through the kindness of Messrs. E. D. Bacon and Albert E. Lawrence, we are able to give the following list of surcharges, on the envelopes of the preceding issue, which have been seen by these gentlemen.

\begin{tabular}{|c|c|}
\hline $\begin{array}{l}\text { Color } \\
\text { of Surcharge. }\end{array}$ & $\begin{array}{l}\text { Measurement } \\
\text { of Surcharge. }\end{array}$ \\
\hline black & • \\
\hline “ & $15 \times I 1 / 2$ \\
\hline “" & I $71 / 2 \times 13 / 4$ \\
\hline “. & I $71 / 2 \times 13 / 4$ \\
\hline “ & Io $3 / 4 \times 1 / 2$ \\
\hline violet & $13 \times I 1 / 2$ \\
\hline black & $20 \times 3$ \\
\hline carmine & $8 \times x^{3} / 4 / 4$ \\
\hline none & none \\
\hline none & none \\
\hline none & none \\
\hline black & $15 \times 21 / 2$ \\
\hline " & $15 \times 13 / 4$ \\
\hline “. & $17 \times 2$ \\
\hline “ & I $21 / 2 \times 13 / 4$ \\
\hline ". & $18 \times 3$ \\
\hline ". & I9x2 \\
\hline “ & $12 \times 2$ \\
\hline “. & \\
\hline violet & I $21 / 2 \times 1 / 2$ \\
\hline black & I $3 \times 11 / 2$ \\
\hline blue & $16 \times x \frac{1}{2}$ \\
\hline black & $171 / 2 \times 13 / 4$ \\
\hline & $20 \times 3$ \\
\hline “ & $151 / 4 \times 13 / 4$ \\
\hline “" & $\operatorname{IXX} 5 / 8$ \\
\hline violet & $103 / 4 \times 13 / 4$ \\
\hline blue & $13 \times 21 / 4$ \\
\hline
\end{tabular}





\begin{tabular}{lc}
\hline & \\
Value. & Number and Date. \\
$25 \mathrm{C}$ & I282 \\
$25 \mathrm{C}$ & I282 \\
$25 \mathrm{C}$ & $\mathrm{I} 78 \mathrm{I}$ \\
$25 \mathrm{C}$ & $188 \mathrm{I}$ \\
$25 \mathrm{C}$ & 2079 \\
$25 \mathrm{C}$ & $2 \mathrm{I} 79$ \\
$25 \mathrm{C}$ & $2 \mathrm{I} 8 \mathrm{O}$ \\
$25 \mathrm{C}$ & $2 \mathrm{I} \mathrm{I}$ \\
$25 \mathrm{C}$ & $238 \mathrm{O}$ \\
$25 \mathrm{C}$ & $248 \mathrm{O}$ \\
$25 \mathrm{C}$ & 2686 \\
$25 \mathrm{C}$ & $278 \mathrm{I}$ \\
$25 \mathrm{C}$ & $308 \mathrm{O}$ \\
$25 \mathrm{C}$ & $328 \mathrm{O}$ \\
$25 \mathrm{C}$ & 3379 \\
$25 \mathrm{C}$ & $368 \mathrm{I}$ \\
$25 \mathrm{C}$ & $388 \mathrm{I}$ \\
$25 \mathrm{C}$ & $398 \mathrm{O}$ \\
$25 \mathrm{C}$ & 3982 \\
$25 \mathrm{C}$ & 4078 \\
$25 \mathrm{C}$ & $408 \mathrm{O}$ \\
$25 \mathrm{C}$ & 4278 \\
$25 \mathrm{C}$ & $438 \mathrm{O}$ \\
$25 \mathrm{C}$ & $448 \mathrm{I}$ \\
$25 \mathrm{C}$ & $468 \mathrm{I}$ \\
$25 \mathrm{C}$ & $508 \mathrm{I}$ \\
$25 \mathrm{C}$ & $54 \% 9$ \\
&
\end{tabular}

Tepic

S. L. Potosi

Saltillo

Puebla

Pachuca

(“)

Oaxaca

Morelia

Merida

Mazatlan

$\mathrm{La} \mathrm{Paz}$

(Jilotepic)

Jalapa

Guanajuato

Durango

Chihuahua

Chiapas

Cordova

C. Guzman

Cocula

Cuantitlan

(Campeche)

Mexico (in half circle)

Issue of 1879 .

$\begin{array}{rr}4 \mathrm{c} & 279 \\ 4 \mathrm{C} & 379 \\ 4 \mathrm{C} & 479 \\ 4 \mathrm{C} & 1979 \\ 4 \mathrm{C} & 2279 \\ 4 \mathrm{c} & 2479 \\ 4 \mathrm{C} & 3879 \\ 4 \mathrm{C} & 5279 \\ 4 \mathrm{C} & 5479 \\ 4 \mathrm{C} & 5480\end{array}$

Issue, March, I88o.

$\begin{array}{rrl}\text { 4c } & 280 & \text { Zacatecas } \\ \text { 4c } & 282 & \text { V" } \\ \text { 4C } & 380 & \text { Vera Cruz } \\ \text { 4c } & 1580 & \text { Tacubaya } \\ \text { 4c } & 1780 & \text { S. L. Potosi } \\ \text { 4C } & 1982 & \text { Queretaro } \\ \text { 4C } & 2780 & \text { Mazatlan } \\ \text { 4C } & 4280 & \text { Cordova } \\ \text { 4c } & 498 \mathrm{I} & \text { Colima } \\ \text { 4c } & 5480 & \text { Mexico (in half circle) }\end{array}$

Issue April, I882 (surcharged " Habilitado").

Zacatecas
(Vera Cruz)
Ures
Queretaro
Orizava
(Morelia)
Durango
Aguascalientes
Mexico (in half circle)

\begin{tabular}{|c|c|}
\hline $\begin{array}{c}\text { Color } \\
\text { of Surcharge. }\end{array}$ & $\begin{array}{l}\text { Measurement } \\
\text { of Surcharge. }\end{array}$ \\
\hline violet & $\begin{array}{l}61 / 2 \times 2 \\
81 / 2 \times 11 / 4\end{array}$ \\
\hline black & $\begin{array}{l}\text { I } 53 / 4 \times 23 / 4 \\
\text { I } 41 / 2 \times 2\end{array}$ \\
\hline “، & $12^{1 / 2} \times 2$ \\
\hline “" & $15 \times 13 / 4$ \\
\hline none & none \\
\hline black & $15 \times 13 / 4$ \\
\hline blue & $15 \times 2$ \\
\hline black & I $4 \times$ (varies) \\
\hline “" & $13 \times 2$ \\
\hline violet & $\begin{array}{l}\text { I } 71 / 2 \times 2 \\
\text { II } 1 / 4 \times 21 / 2\end{array}$ \\
\hline none & none \\
\hline black & $15 \times 3$ \\
\hline blue & $18 \times 11 / 2$ \\
\hline vermillion & $18 \times 21 / 4$ \\
\hline black & $\begin{array}{l}18 \times 3 \\
18 \times 3\end{array}$ \\
\hline blue & $14 \times 13 / 4$ \\
\hline “ & $14 \times 1 \frac{3}{4}$ \\
\hline black & $\begin{array}{l}131 / 2 \times 11 / 2 \\
15 \times 11 / 2\end{array}$ \\
\hline ، & $15 \times 11 / 2$ \\
\hline “" & $19 \times 2$ \\
\hline $\begin{array}{l}\text { none } \\
\text { black }\end{array}$ & none \\
\hline black & $16 \times 17 / 8$ \\
\hline $\begin{array}{l}\text { none } \\
\text { black }\end{array}$ & $\begin{array}{l}\text { none } \\
7 \times 2\end{array}$ \\
\hline "1 & $14 \times 1 / 2$ \\
\hline blue & $17 \times 2$ \\
\hline none & none \\
\hline black & I $81 / 2 \times 2$ \\
\hline “ & $27 \times 2$ \\
\hline “" & \\
\hline 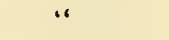 & \\
\hline
\end{tabular}

\begin{tabular}{|c|c|}
\hline blue & $16 \times 17 / 8$ \\
\hline $\begin{array}{l}\text { carmine-lake } \\
\text { black }\end{array}$ & $\begin{array}{l}16 \times 11 / 2 \\
18 \times 11 / 2\end{array}$ \\
\hline blue & I $41 / 2 \times 11 / 2$ \\
\hline black & $\begin{array}{l}14 \times 11 / 2 \\
14 \times 11 / 2\end{array}$ \\
\hline “" & I $71 / 2 \times 2$ \\
\hline “ & I $4 \times I 3 / 4$ \\
\hline & $12 \times 2$ \\
\hline
\end{tabular}


Value. Number and Date. Issue, end $\mathrm{r} 882$.

$5 \times 5 \mathrm{c}$

$5 \times 5 \mathrm{c}$

$5 \times 5 \mathrm{c}$

$5 \times 5 \mathrm{C}$
983
2882

2882

5483
District Name.

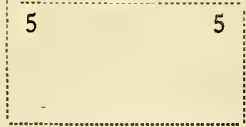

Toluca

Matamoros $\left\{\begin{array}{l}\text { probably } \\ \text { the same }\end{array}\right\}$

Mexico (in half circle)

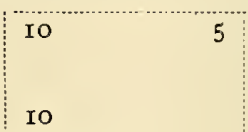

\begin{tabular}{|c|c|}
\hline $5 \times I O X I O C$ & 283 \\
\hline $5 \times 10 \times 100$ & 283 \\
\hline $5 \times 10 \times 100$ & 383 \\
\hline $5 \times 10 \times 10 c$ & 683 \\
\hline $5 \times \operatorname{rox} 100$ & 683 \\
\hline $5 \times 10 \times I O C$ & 983 \\
\hline $5 \times I O X I O C$ & 1682 \\
\hline $5 \times 10 \times r o c$ & I 782 \\
\hline $5 \times 10 x I O C$ & 1883 \\
\hline $5 \times I O x I O C$ & 2083 \\
\hline $5 \times 10 \times 100$ & 2183 \\
\hline $5 \times 10 \times 100$ & 2283 \\
\hline $5 \times \operatorname{rox} 100$ & 2383 \\
\hline $5 \times I 0 \times 10 c$ & 2582 \\
\hline $5 \times 10 x I O C$ & 2783 \\
\hline $5 \times 10 \times 10 c$ & 2783 \\
\hline $5 \times 10 \times 10 c$ & 2882 \\
\hline $5 \times 10 \times 100$ & 3083 \\
\hline $5 \times 10 \times 100$ & 3182 \\
\hline $5 \times 10 \times 100$ & 3382 \\
\hline $5 \times 10 \times 100$ & 3783 \\
\hline $5 \times 10 \times 10 c$ & 4282 \\
\hline $5 \times I O x I O C$ & 4582 \\
\hline $5 \times 10 x 10 c$ & 4883 \\
\hline $5 \times 10 \times 100$ & 5483 \\
\hline $5 \times 10 \times 100$ & $\mathrm{n}$ \\
\hline
\end{tabular}

$5 \times 5 \times 5 \times 100$

$\begin{array}{lll}5 & 5 \\ 5 & 10\end{array}$

Zacatecas

Vera Cruz (too faint to measure)

Tula de T.

(Tula de T.)

Toluca.

(Tabasco)

S. L. Potosi

(Saltillo)

Puebla

Pachuca

Orizaba

Oaxaca

Monterrey

Mazatlan

Matamoros

La Paz

Lagos

Jalapa

Guadalajara

Cordova

Cuernavaca

C. Bravos

Mexico (in half circle)

none

Huejutla (left upper corner cut off)
Color

of Surcharge.

Measurement of Surcharge.

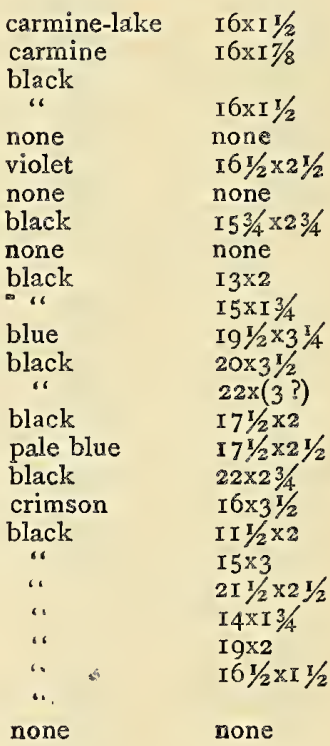

$\begin{array}{cc}\text { violet } & 15 \times 28 / 2 \\ \text { black } & 22 \times 23 / 4 \\ \text { "f } & 221 / 2 \times 3 \\ \text { ". } & \end{array}$

none

blue

I $6 \mathrm{xII} / 2$

Issue of 1883 .

Yellow wove paper-Size $138 \times 78$

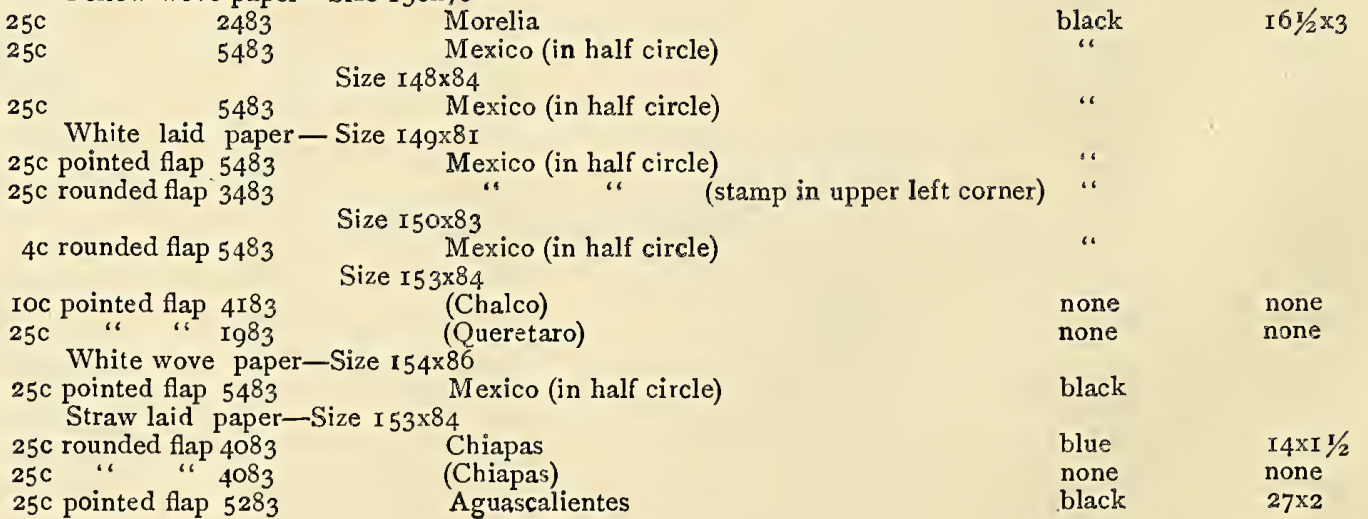



June, I 884 .

Stamp of same type as preceding issue on white laid paper, watermarked eagle surrounded by "Admon, General de Correos Mexico" in circle. Name of maker, "Waterlow \& Sons, Limited, London Wall, London," to left of flap.

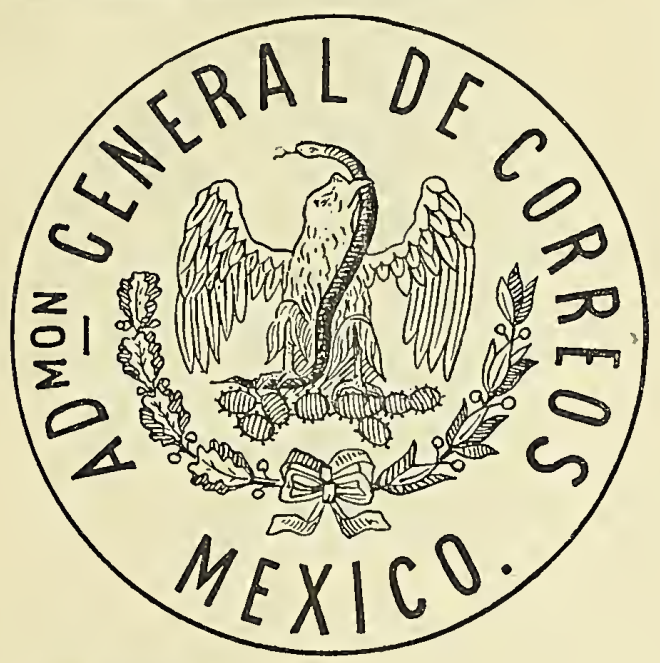

Size $145 \times 89 \mathrm{~mm}$.

3158 IOc green

Lariety: With 3 additional stamps, of which two are inverted in lower corners, and the other one embossed on the back of the envelope.

3I59 IOxIO $\mathrm{x}$ IOxIOC green

From this date the envelopes are no longer surcharged with district name, number and date.

July, I 884 .

Siamp of same type as the adhesives of corresponding date, embossed in upper right corner on white laid paper; same watermark as in preceding issue, and name of maker (Waterlow \& Sons, etc.), on left flap.

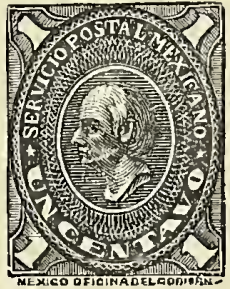

Size $145 \times 89 \mathrm{~mm}$.

3160 IOc vellow green

3I6I Ioc blue green

Variety: Name of maker on right flap.

3162 Ioc yellow green

January, I 885 .

Same type, paper and watermark as preceding issue. Name of makers, Waterlow \& Sons, Ltd., London Wall, London, on left flap.

Size $145 \times 89 \mathrm{~mm}$.

3163 4c vermilion

$3164 \quad 5 \mathrm{c}$ ultramarine

3165 6c brown

3166 Ioc orange

3167 I2c black brown

$316820 c$ green

Varieties:

a. Without makers' name.
3169

3170

3171

3173
3174

2oc green

b. Last word (London) of maker's name and address omitted.

$3175 \quad 4 \mathrm{C}$ vermilion

3176 5c ultramarine

3177 6c brown

3178 IOc orange

3179 I2c black brown

3I 80 20c green

c. Stamp inverted in lower left corner.

3I 8 I $4 \mathrm{c}$ vermilion

d. Without stamp.

3I 82 no value, no color

I 886.

Stamp of same type as adhesives of corresponding date, embossed on white laid paper. Watermalked same as preceding issue and name of maker on left flap.

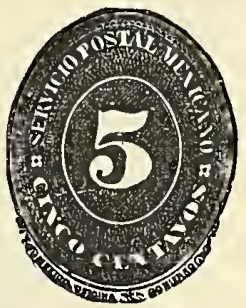

Size $145 \times 89 \mathrm{~mm}$

3183 Ioc brown violet

Varieties:

a. Additional stamp in upper right corner on back of envelope.

3I 84 Ioc brown violet

b. Without maker's name imprinted.

3 I 85 IOc brown violet

I 888.

Same type, paper, etc., as preceding issue. Size $145 \times 89 \mathrm{~mm}$.

3I 86 5c ultramarine

3187 Ioc scarlet

Varieties: Without maker's imprint.

3I $88 \quad 5 \mathrm{c}$ ultramarine

3I 89 IOc scarlet

September, I 889 .

Stamp of same type as preceding issue, bag-shaped envelope, with flap at left; Mexican Coat of Arms, with bandrol below inscribed SERVICIO POSTAL MEXICANA in upper left corner. There are two types of this Coat of Arms, in the first one the extremities of the bandrol are shaded and the central cactus is lined diagonally; in the second type the extremities of the bandrol are white and the central cactus has diagonally crossed lines.

Size $158 \times 91 \mathrm{~mm}$.

\begin{tabular}{|c|c|c|}
\hline $3 \mathrm{IgO}$ & Coat of Arms & type I \\
\hline $319 I$ & $4 \mathrm{c}$ & "II \\
\hline 3192 & $5 \mathrm{c}$ light ultramarine & " I \\
\hline 3 I 93 & $5 \mathrm{c}$ " & " II \\
\hline 3194 & $5 \mathrm{c}$ deep ultramarine & “I \\
\hline 3195 & $5 \mathrm{c} "$ " & "II \\
\hline 3196 & $6 \mathrm{c}$ scar'et & \\
\hline 3197 & $6 c " 1$ & “II \\
\hline $\begin{array}{l}319 / \\
3198\end{array}$ & IOC & “ I \\
\hline 3199 & IOC & "II \\
\hline 3200 & I $2 \mathrm{C}$ & \\
\hline
\end{tabular}


3201 I2c scarlet $320220 \mathrm{c}$ scarlet
Coat of Arms type II

OFFICIAL ENVELOPES.

I884.

Stamp of same type as official adhesive stamps of same date, embossed in upper right corner on various paper :

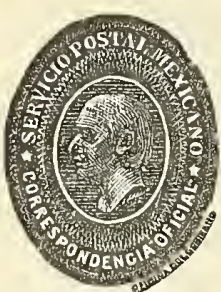

$I^{\circ}$ White laid paper.

a. Size I82xirg mn.

3300 briglit red

b. Size r $96 \times 133 \mathrm{~mm}$

330r bright red

c. Size $240 \operatorname{mrr} 5 \mathrm{~mm}$

3302 bright red

\section{For Inland Postage}

WRAPPERS.
$320320 c$ scarlet Coat of Arms type II

d. Size $250 x \mathrm{rr} 6 \mathrm{~mm}$

3303 bright red

e. Size $260 \times 176 \mathrm{~mm}$.

3304 bright red

$2^{\circ}$ Yellow laid paper.

Size 226x99 $\mathrm{mm}$.

3305 bright red

$3^{\circ}$ White wove paper.

a. Size 254 XI I $3 \mathrm{~mm}$.

3306 bright red

b. Size r 7oxrr $7 \mathrm{~mm}$.

3307 bright red.

$4^{\circ}$ Buff wove paper.

Size 256 XI I $3 \mathrm{~mm}$.

3308 bright red

$5^{\circ}$ Straw paper.

Size ? $\mathrm{mm}$.

3309 bright red

$6^{\circ}$ Orange laid paper.

Size 222x99 mm.

33 Io bright red.

July, Ist, I886.

Label and inscriptions typographed in black and stamp embossed in color on orange wove paper, flap at right with truncated ends gummed.

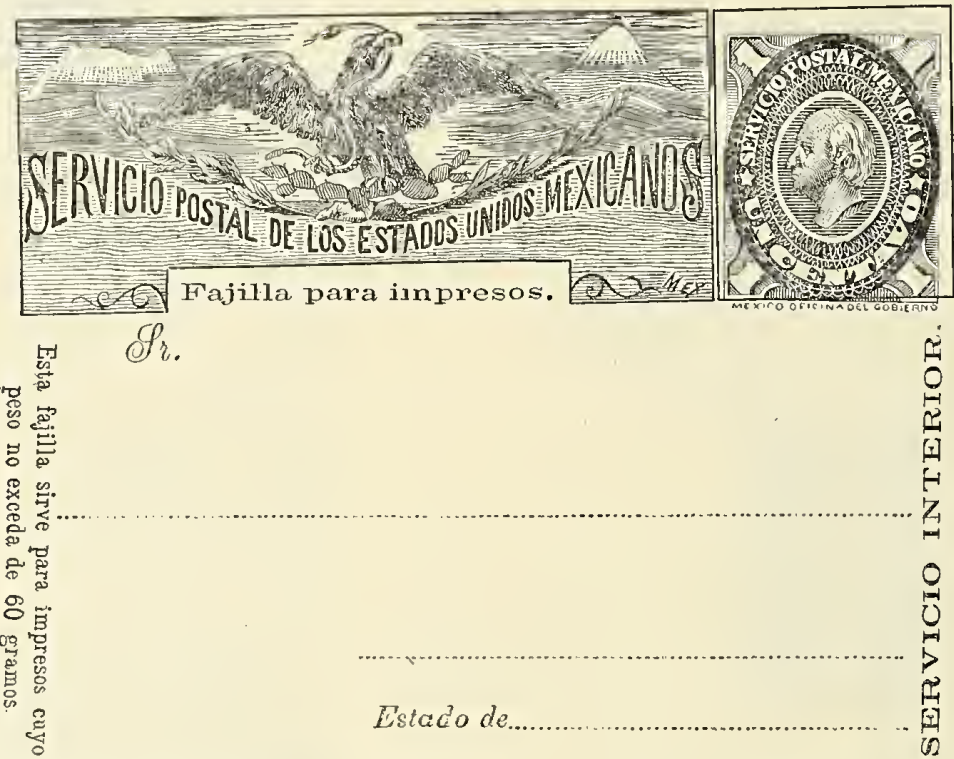

Size. $245 \times 80 \mathrm{~mm}$

A. The first line for address is opposite $\mathrm{N}$ of Interior, the and between $\mathrm{R}$ and $\mathrm{V}$ of Servicio, the 3 rd opposite $\mathrm{S}$ of Servicio.

$I^{\circ}$ Groundwork under eagle is lined all through.

$340 \mathrm{I} \quad$ Ic $(6 \circ \mathrm{og})$ green and black

Varieties:

a. Flap to left.

3402 Ic (6ogr) green and black

b. Flap gummed on face.

3403 I c (6ogr) green and black

$2^{\circ}$ Groundwork under eagle is plain.

3404 Ic $(60 \mathrm{or})$ green and black

$34052 \mathrm{2c}$ (1 20gr) red and black

Varieties:

a. Flap to left.
3406 Ic (6ogr) green and black $3407 \quad 2 \mathrm{c}(\mathrm{1} 2 \mathrm{ggr})$ red and black

b. I abel and inscription printed on both sides.

$3+082 \mathrm{c}(\mathrm{r} 20 \mathrm{gr})$ red and black

c. Left side of top and bottom of first I of Interior broken and break in label between I and $\mathrm{C}$ of Mexicanos, extending vertically upwards and diagonally downwards to left.

3409 rc (6ogr) green and black

34I0 2c (I20gr) red and black

d. Same as "c" with error in inscription

"I 20 gramos" instead of "6o gramos". 34 II IC (I20gr) green and black

e. Same as "c" but flap to left.

$34 \mathbf{1} 2$ 2c (120gr) red and black 

f. Break in upper label below A of Mexicanos.

3413 Ic (6ogr) green and black

$34 \mathrm{I} 4 \quad 2 \mathrm{c}$ (I20gr) red and black

g. Same as "f $f$ " but flap to left.

$34152 c$ (I20gr) red and black

$h$. Break in label between I and $C$ of Mexi-

canos extending upwards to laurel branch.

$34 \mathrm{I} 6$ Ic (60gr) green and black

34I 72 (I 20gr) red and black

i. Same as "h" but flap to left.

$34 \mathrm{r} 8 \quad$ Ic (6ogr) green and black

341920 (I20gr) red and black

j. Flap gummed on face.

3420 Ic (6ogr) green and black

k. Without stamp and flap ungummed and not truncated.

342 I (6ogr) black

B. The first line for address is opposite $\mathrm{N}$, the and opposite $\mathrm{V}$, and the 3 rd opposite $\mathrm{E}$; break under A of Mexicanos in label. 3422 Ic (6ogr) green and black

\section{November, 1886 .}

Inscriptions typographed in black and stamp embossed in color on orange wove paper; flap with truncated ends and gummed to right. Size $245 \times 80 \mathrm{~mm}$.

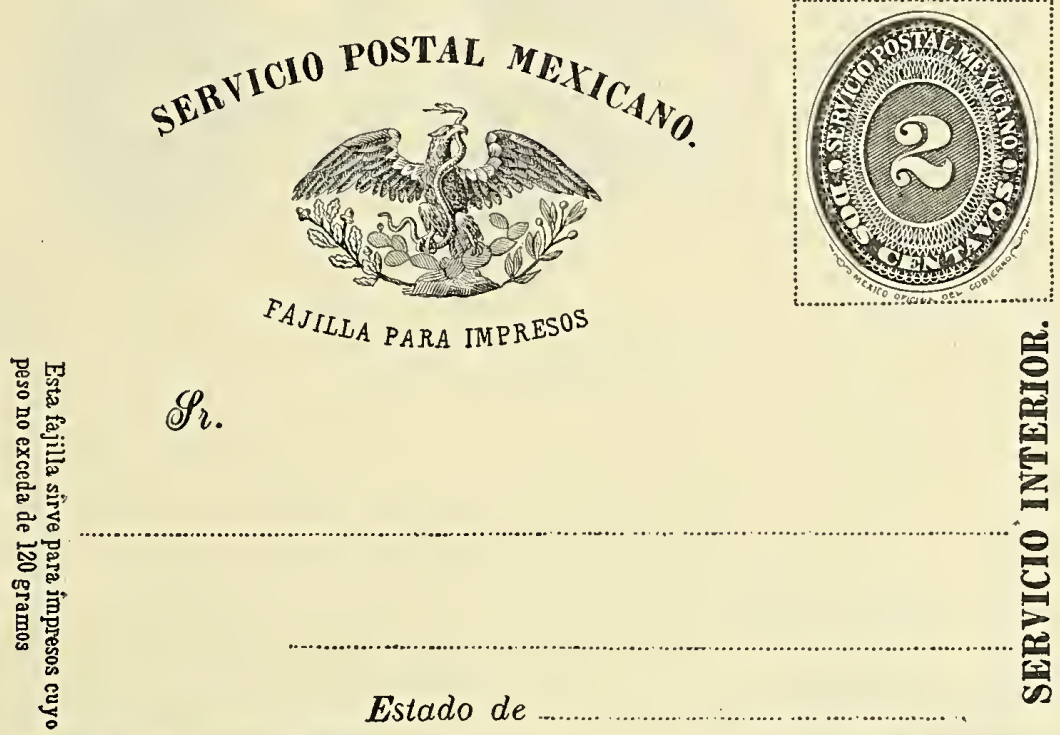

A. Stamp embossed in dotted reciangle; the first line for address is opposite I of Servicio and the 2nd opposite E.

3432 Ic (6ogr) green and black

$3433 \quad 2 c(120 g r)$ and black

Varieties:

a. Error in inscription.

3434 Ic (120gr) green and black

$3435 \quad 2 c(60 \mathrm{gr})$ red and black

b. Double impression of stamp, the second one colorless.

34362 2x2c (I20gr) red and black

B. Stamp embossed in ruled rectangle; the first line for address is between $\mathrm{I}$ and $\mathrm{O}$ of Servicio, the second opposite R.

3437 Ic $(60 \mathrm{gr})$ green and black

Varieties:

a. Error: 2c stamp impressed instead of Ic stamp.

3438 2c (6ogr) red and black

b. Flap to left.

3439 Ic $(6 \circ g r)$ green and black

$3440 \quad 2 c(60 g r)$ red and black

c. No puncluation after 'Gramos'.

344I Ic (6ogr) green and black
Varieties:

a Error: 2c stamp impressed instead of Ic. $3423 \quad 2 c(6 \circ g r)$ red and black

b. Flap to left.

3424 Ic (6ogr) green and black

$34252 \mathrm{c}(6 \circ \mathrm{gr})$ red and black

c. The first line for address is between I and $\mathrm{N}$, the 2nd opposite $\mathrm{V}$ and the 3 rd between $\mathrm{S}$ and $\mathrm{E}$.

3426 Ic (6ogr) green and black

Varieties:

a. Label and inscription printed on both sides.

3427 Ic (6ogr) green and black

b. Inscription at left is colorless.

$3+28$ Ic (6ogr) green and black

c. Error: $2 \mathrm{c}$ stamp impressed instead of Ic.

3429 2c (6ogr) green and black

d. First line for address is between $I$ and $\mathrm{N}$, the 2 nd between $\mathrm{R}$ and $\mathrm{V}$; break between $\mathrm{I}$ and $\mathrm{C}$ of Mexicanos.

$3430 \quad$ Ic (6ogr) green and black

343I 2c (I 20gr) red and black 
A. First line for address is just above $\mathrm{O}$ of Servicio and the second opposite $R$.

$3450 \quad$ Ic (6ogr) green and black

345 I 2c (I2Ogr) red and black

I arieties:

a. Double impression of stamp, one next to the other.

3452 ICXIC (6ogr) green and black

3453 Icx Ic (I 20gr) green and black

b. Stamp inverted to the left of rectangle.

3454 Ic (6ogr) green and black

c. Double impression of inscription, eagle excepted.

3455 Ic (6ogr) green and black

d. G of "Gramos" is under M of 'Impresos'.

3456 Ic (6ogr) green and black

November, I 892 .

Similar to preceding issue, without dotted space for stamp, instructions at sides in capitals; inscriptions and instructions lithographed in color and stamp embossed in color on buff paper glazed on the surface; flap to left, gummed, truncated ends. Size $248 \times 78 \mathrm{~mm}$.

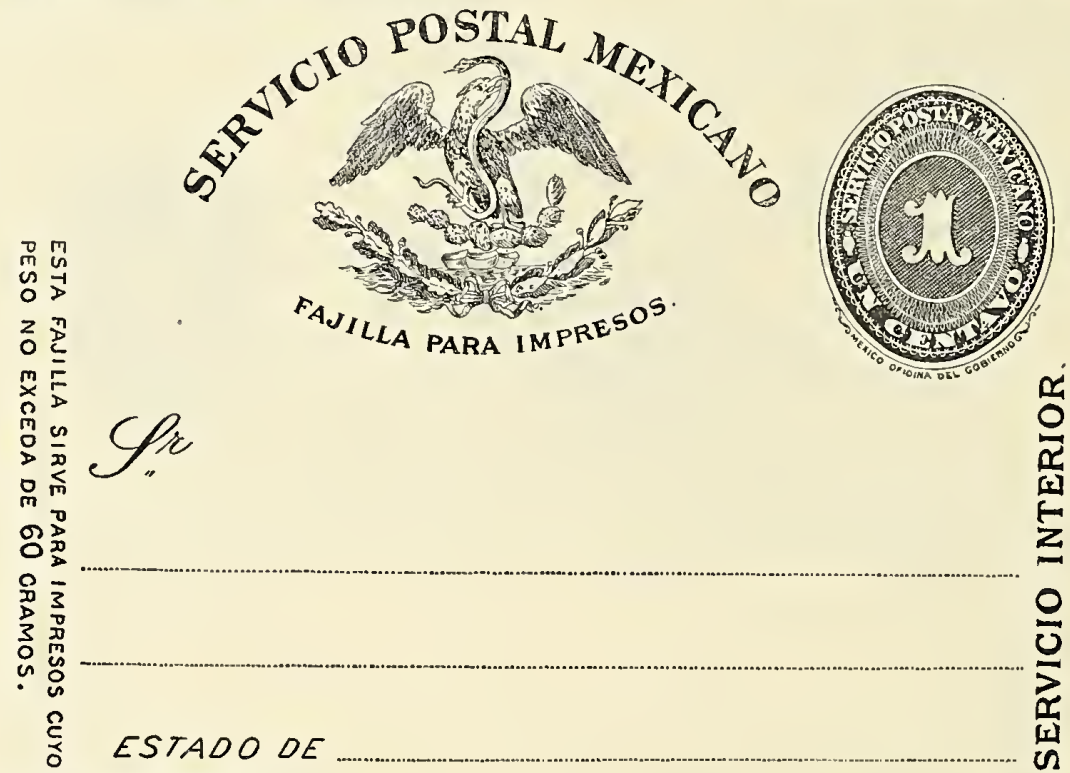

3464 Ic green, blue inscription

3465 2c carmine, rose inscription

\section{For Universal Postal Union.}

July ist, I 886

Label and inscription typographed in black, and stamp embossed in color on orange wove paper; flap with truncated ends and gummed at left. Size $245 \times 80 \mathrm{~mm}$.

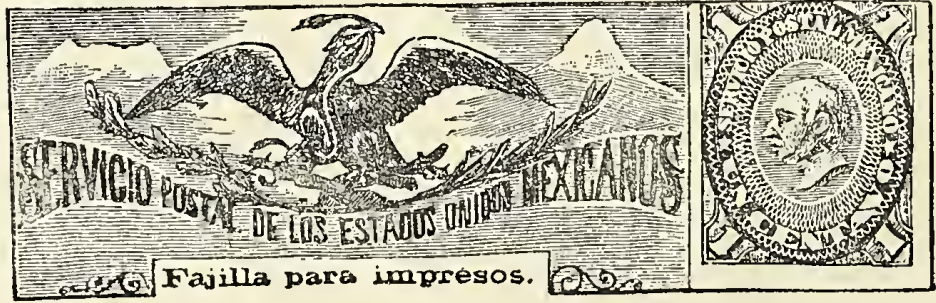

UNION POSTAL UNIVERSAL.--UNION POSTALE UNIVERSELLE. MEXICO.-- MEXIQUE. 


A. Break in label under A of Mexicanos. $3564 \quad 2 \mathrm{c}$ red and black

B. Break in label between $I$ and $C$ of Mexicanos.

a First line for address ends before () of Postal.

3565 Ic green and black

$3566 \quad 2 \mathrm{c}$ red and black

I arieties:

$I^{\circ}$ Flap at left.

3567 Ic green and black

$3568 \quad 2 \mathrm{c}$ red and black

$2^{\circ}$ Second line for address ends before

$S$ of Postal.

3569 Ic green and black

$3570 \quad 2 \mathrm{c}$ red and black

Variety: Flap to left.

$357 \mathrm{I} 2 \mathrm{c}$ red and black

November, I 886.

Inscriptions typographed in black, and stamp embossed in color on orange wove paper; flap to right, truncated and gummed. Size $245 \times 80 \mathrm{~mm}$.
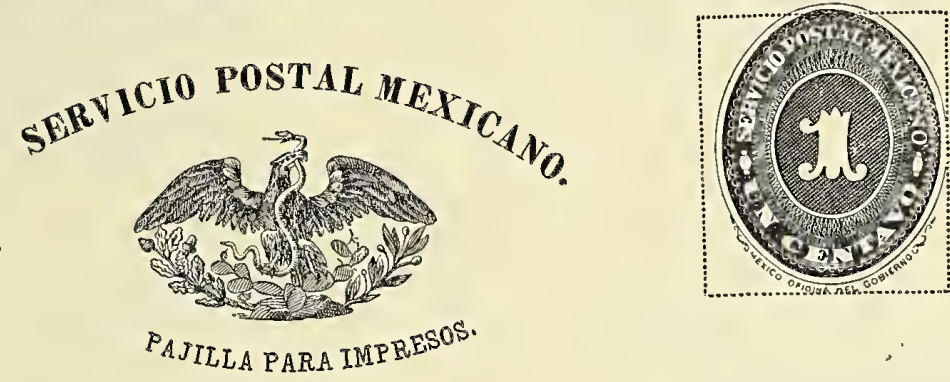

UNION POSTAL UNIVERSAL,...UNION POSTALE UNIVERSELLE. MEXICO...MEXIQUE $3^{\circ}$ First line for address ends before $\mathrm{T}$.

Ic green and black

$2 \mathrm{c}$ red and black

$4^{\circ}$ First line for address ends under $\mathrm{S}$.

$U$. and $\mathrm{N}$. of Union Postal are broken.

3574 Ic green and black

$35752 \mathrm{c}$ red and black

$5^{\circ}$ The first line ends under $P$.

$3576 \quad$ Ic green and black

$3577 \quad 2 \mathrm{c}$ red and black

$6^{\circ}$ The first line of address ends under $S$, and the 2nd $N$ of Union is over $M$ of Mexique.

3578 Ic green and black

Variety: Flap is not truncated.

$3580 \quad 2 \mathrm{c}$ red and black
$3579 \quad 2 \mathrm{c}$ red and black
A. Stamp embossed in dotted rectangle. Mexique puntuated; the first line for address ends in $T$ of Postal.

358I Ic green and black

$3582 \quad 2 \mathrm{c}$ red and black

Varieties:

a. Without stamp.

3583 black

b. Stamp inverted.

$3584 \quad 2 \mathrm{c}$ red and black

c. Double impression of stamp, the first one colorless.

$3585 \quad 2 \mathrm{c} \times 2 \mathrm{c}$ red and black

d. Double impression of stamp, both in color.

$3586 \quad 2 \mathrm{cx} 2 \mathrm{c}$ red and black

e. Flap to left.

$3587 \quad$ Ic green and black

3588 2c red and black

B. Stamp in dotted rectangle, Mexique is not punctuated.

3589 Ic green and black

$359^{\circ} \quad 2 \mathrm{c}$ red and black

Varieties.

Flap to left.

$359 \mathrm{I}$ Ic green and black

3592 2c red and black

C. Stamp in ruled rectangle. 
b. Flap to left.

$3606 \quad 2 \mathrm{c}$ red and black

c. Flap to left, ungummed.

3607 Ic green and black $3^{\circ}$ Same as " $2^{\circ}$ "; I of Mexique is under second $\mathrm{N}$ of Union Postal.

$3608 \quad$ Ic green and black

\section{PROVISIONAL ISSUES.}

Issued during the Revolution of $1867-68$ by the postmasters of various cities immediately after the withdrawal of the Imperial troops.

\section{Campeche.}

\section{I867.}

Hand stamped in black and blue on white wove paper; the seal at top in blue, the séal below in black and the figure of value in blue. The spaces for the stamps on the sheets were ruled out with pencil.

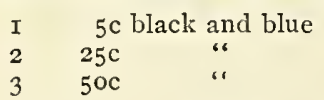

Number $I$ is given on the authority of Heitman's Handbuch, where it is stated that there is one in the collection of Mr. Philip de Ferrary.

\section{I867,}

Typographed in black on colored wove paper. Size $25 \times 17 \mathrm{~mm}$.

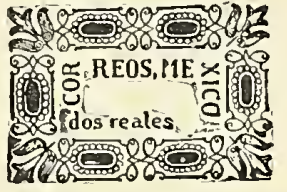

I $\quad 1 / 2$ (Medio) $\mathrm{r}$ black, blue paper

I 868 .

Hand stamped in black on various papers. Size $25 \mathrm{~mm}$., in diameter.

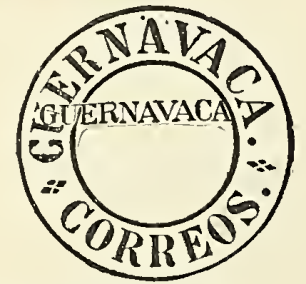

I. Without district name.

White wove paper.

I867.

Hand stamped in black on various papers. Size $2 \mathrm{Imm}$. in diameter; the spaces for the stamps on the sheets were ruled off with pencil. The die used to print these stamps was the ordinary cancelling stamp of the of fice, with the date in the centre replaced by the value and date. Whenever a new supply of certain values was needed the value had to be reset, thus causing slight variations in the relative positions of punctuation and the

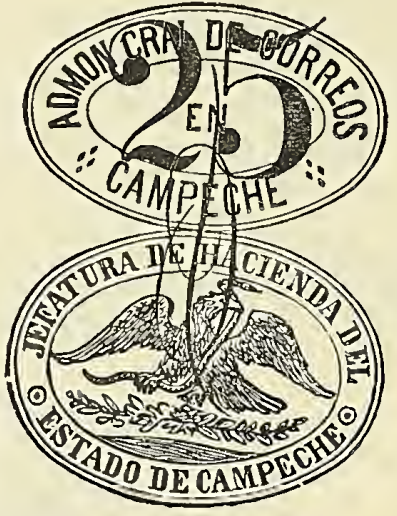

Chiapas

2 I (un) r black, light green paper

32 (do.) $\mathrm{r}$ black, light pink paper

44 (cuatro) $r$ black, light pink paper 58 (ocho) $\mathrm{r}$ black, light pink paper

Varieties :

a. 4 reales stamps cut in two, each half being used as 2 reales.

$62 \mathrm{r}$ black, light pink paper (half of 4 reales)

b. 8 reales stamps cut in quarters, each" quarter being used as 2 reales.

7 2r black, pink paper ( $1 / 4$ of 8 reales)

\section{Cuerpavaca}

I (2 reales) black

II. With district name.

$I^{\circ}$ White wove paper.

2 (2 reales) black

$2^{\circ}$ Bluish wove paper.

3 (2 reales) black

III. With two district names.

White wove paper.

4 (2 reales) black

Mr. Moens calalogues as doubtful another Cuernavaca of similar design but oval instead of circular. We must confess that in our mind there is also much doubt about the authenticity of the circular one and we should not be surprised if some day it turns out to be a humbug.

\section{Guadalajara.}

letters; these latter being sometimes below and at other times above the line.

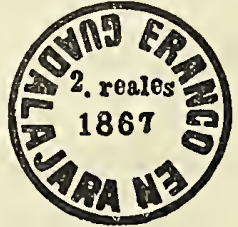




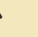




\section{•}


I. Wove paper.

$1 / 2 \mathrm{r}$ (Medio) black, white paper Ir (Un real) black, blue paper Ir (un real) black, dark blue paper Ir (un real) black, white paper Ir (un real.) black, gray blue paper Ir (un real.) black, rose paper 2 r (2 reales.) black, white paper 2r (2 reales.) black, yellow green paper

$92 \mathrm{r}(2$ reales.) black, green paper 2r $(2$ reales.) black, rose paper 2 r 2 reales.) black, dark blue paper $4 \mathrm{r}$ (4 reales.) black, white paper $4 \mathrm{r}$ ( 4 reales.) black, rose paper 4r (4. reales) black, lilac blue paper Ip (Un peso.) black, lilac paper Ip (un peso.) black, lilac paper Ip (un peso.) black, rose paper Varieties:

a. Perforated in scallops.

I $1 / 2 \mathrm{r}$ (Medio) black. white paper

I9 2 r (2 reales.) black, green paper

We catalogue these two on the authority of

Mr. J. B. Moens.

b. Perforated in points

$20 \quad 1 / 2$ r (Medio) black, white paper Ir (Un real) black, blue paper Ir (un real) black, dark blue paper Ir (un real) black, white paper Ir (un real) black, gray blue paper 2 r (2 rea'es,) black, green paper $2 r$ ( 2 reales.) black, rose paper $4 \mathrm{r}$ (4. reales) black, lilac blue paper $4 \mathrm{r}(4$ reales.) black, rose paper Ip (Un peso.) black, lilac paper Ip (un peso.) black, lilac paper Ip (un peso.) black, rose paper

' $I$ ' of 'I 867 ' missing.

2r (2 reales $)$ black, rose paper

d. ' $s$ ' inverted. 4r (4. reales) black, lilac blue paper

Space between 6 and 7 . 2r (2 reales.) black, white paper 2 r 2 reales.) black, green paper

Half of 2 reales stamp used as I real. Ir black, green paper (half of 2 reales.)

. Half of 4 reales stamp used as 2 reales. $2 \mathrm{r}$ black, rose paper (half of 4 reales.)

II. Square quadrille paper.

38 Ir (Un real.) black, blue paper $2 \mathrm{r}(2$ reales.) black, green paper 2r (2. reales) black, rose paper 2r (2. reales.) black, rose paper $4 \mathrm{r}$ (4. reales) black, lilac blue paper $4 \mathrm{r}$ (4. reale) black, white paper Ip (Un peso.) black, rose paper Ip (Un peso.) black, lilac piper Ip (Un peso.) black, claret paper Ip (Un peso) black, lilac paper Ip (Un peso.) black, deep blue paper

Varieties:

a. Perforated in points.

Ir (Un real.) black, blue paper 2r (2 reales.) black. green paper 2r (2. reales) black, rose paper 2r (2. reales.) black, rose paper $4^{\mathrm{r}}$ (4. reales) black, lilac blue paper $4 \mathrm{r}$ (4. reales) black, white paper Ip (Un peso.) black, lilac paper

I of I 867 missing. 2r (2 reales.) black, green paper

IIalf of 2 reales used as I real. Ir black, green paper (half of 2 reales)

III. Oblong quadrille paper.

58 Ir (Un real.) black, lilac blue paper

59 Ir (Un real.) black, white paper

$60 \quad 4 \mathrm{r}$ (4. reales) black, lilac blue paper
Variety: Perforated in points.

6I Ir (Un real.) black, lilac blue paper

IV. Finely laid paper.

62 2r (2. reales) black, rose paper

632 2r (2 reales.) black, green paper

$64 \quad 4 \mathrm{r}$ (4. reales) black, lilac blue paper

654 (4. reales) black, rose paper

a. Perforated in points.

66 2r (2. reales) black, rose paper

$67 \quad 2 \mathrm{2}$ ( 2 reales.) black, green paper

b. I of I 867 missing.

68 2r (2. reales) black, rose paper

V. Wide laid paper, blue ruled.

694 r (4. reale) black, white paper

Of this we have seen only one specimen which is in the collection of Dr. W. C. Bowers, and is an unquestionably genuine stamp.

\section{Laid batonne paper.}

Ir (Un real.) black, green paper Ir (Un real) black, lilac blue paper 2r (2. reales) black, green paper 2r (2. reales) black, rose paper $4 \mathrm{r}$ (4. reales) black, lilac blue paper $4 \mathrm{r}$ (4. reales) black, white paper Ip (Un peso.) black, lilac paper Ip (Un peso.) black, rose paper

\section{Varieties:}

a. Perforated in points.

78 Ir (Un real.) black, lilac blue paper

79 Ir (Un real.) black, green paper

$80 \quad 2 \mathrm{r}$ (2. reales) black, green paper

8 I 2r (2. reales) black, rose paper

824 4r (4. reales) black, lilac blue paper

83 Ip (Un peso.) black, lilac blue paper

b. "s" of "reales" omitted.

842 2r (2. reale) black, rose paper

c. I of $I 867$ omitted,

854 (4. reales) black, white paper

VII. Wove batonne paper

862 2r (2. reales) black, rose paper

I 868.

Same type, impression and paper as preceding issue, the date only being changed.

I. Wove paper.

87 . 2r (2 reales.) black, lilac paper 88 2r 2 reales.) black, rose paper Varieties:

a. Perforated in points

$89 \quad 2$ r (2 reales.) black, lilac paper

$90 \quad 2 \mathrm{r}(2$ reales.) black, rose paper

b. " 2 " inverted.

9I 2r (2 reales.) black, lilac paper

c. Figure of value omitted.

92 (reales.) black, lilac paper

II. Laid batonne paper.

93 Ir (un real.) black, green paper

942 2r (2 reales.) black, lilac paper anieties.

a. Perforated in points.

95 Ir (un real.) black, green paper b. I 863 instead of I 868 .

96 Ir (un real.) black, green paper

972 2r $(2$ reales.) black, lilac paper c. Without date.

982 2r (2 reales.) black, lilac paper

d. Figure of value omitted.

99 (re lles.) black, lilac paper

III. Square quadrille paper.

Ioo 2 (2 2 reales.) black, lilac paper

Variety: Perforaled in points.

IOI $2 \mathrm{r}(2$ reales. $)$ black, lilac paper

IV. Laid paper.

IO2 Ir (un real.) black, green paper

IO3 2 r (2 reales.) black, rose paper 
IO4 2 r (2 reales.) black, lilac paper

Varieties:

a. Perforated in points.

I05 Ir (un real.) black, green paper

Io6 2r (2 reales.) black, rose paper

Double impression, one over the other.

I07 Ir (un real.) black, green paper

Io3 2 r (2 reales.) black, lilac paper

Triple impression.

I09 Ir (un real.) black, green paper

d. Double impression of the figure of value, one next the other.

I IO (22 reales) black, lilac paper

e. Nu instead of un.

I I Ir (nu real.) black, green paper

f. I 860 instead of 1868

I 22 2 (2 reales.) black, lilac paper

g. No 's' in 'reales'.

I 32 2r (2 reale) black, rose paper

h. Spacing between value and date wider than usual $-21 / 2 \mathrm{~mm}$. instead of $\mathrm{I} 1 / 2 \mathrm{~mm}$.

I 4 Ir (un real) black, green paper

Thi $;$ is the only stamp in which the spacing differs materially.

No Medio real was issued in 1868 . We have, however, seen one in a collection made

Monterey.

I867.

Hand stamped in black on blue paper. Size $31 \times 21 \mathrm{~mm}$.

I $5 \mathrm{c} \mathrm{black,} \mathrm{blue} \mathrm{paper.}$

Patzcuar».

I 868.

Hand stamped in black on various papers, without value expressed. Size $22 \mathrm{~mm}$., in diameter.

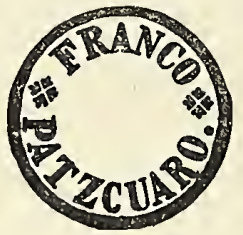

I 867 .

Typographed in black on blue wove paper. Size $21 \times 22 m \mathrm{mn}$.

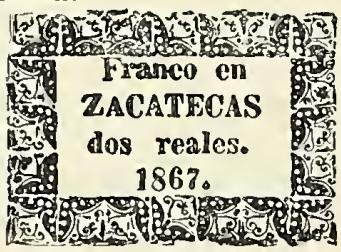

I 856, I 86 I and I867 Issues.

We do not know of any good counterfeits of these issues, but reprints exist with forged surcharges. We have already given the facsimile of the counterfeit surcharge of the 1867 issue in Vol. VI, page 399 of the American Journal of Phrlately. In regard to those of the 1856 and I $86 \mathrm{I}$ issues we refer to the list of surcharges and measurements given in the same volume on page 353 .

I 863 Issue.

There is a very fine counterfeit of the stamps of this issue but it is easily told by the following points:$I^{\circ}$ In the genuine there are four white lines in the stock around the neck of Juarez, while in the counterfeit there are five. up by the Mexican Government for exhibitional purpose, but this was only a counterfeit. Some of the stamps are found on watermarked paper, this, however, is of no special interest, it being only a part of the trade mark of the manufacturer. The Guadalajara stamps were not only used in the Guadalajara office, but some are known with the ancellations: Ciudad Guzman, Tepic, Hosto Tipaquillo, all in the State of Jalisco, and sub-districts of the Guadalajara office. We have also seen the following surcharged "C. Guzman" as illustrated on accompanying plate.

I867 Ir (un real) black, white wove paper 2r 2 reales.) black, white wove paper 2r ( 2 reales.) black, green wove paper

Reprints.

Guadalajara stamps have never been reprinted. The so-called reprints are, it is true, made from the original die, but the value and date were inserted a fterwards and are of different type from the genuine. We illustrate these so-called reprints on the accompanying plate.

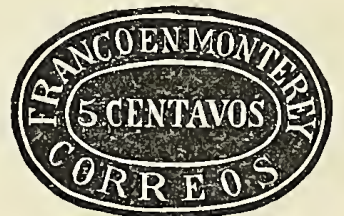

I. Wove paper.

I (2 reales) black, blue paper

II. Oblong quadrille paper.

2 (2 reales) black, blue paper

We have serious doubts about the authenticity of this stamp; this opinion is shared by other authorities, the stamp being according to some of these only a cancellation cut from an old letter and pasted on some other giving it the appearance of of a genuine adbesive.

\section{I real black, blue paper}

22 reales black, blue paper

These stamps also belong to the doubtful class, such as the Cuernavaca and Patzcuaro, and probably are of no more value than the so-called Chalco, Chihuahua, Morelia, Oajaca, Queretaro and Vera Cruz, all late discoveries, and now recognized by most authorities as frauds.

\section{COUNTERFEITS}


GUADALAJARA.
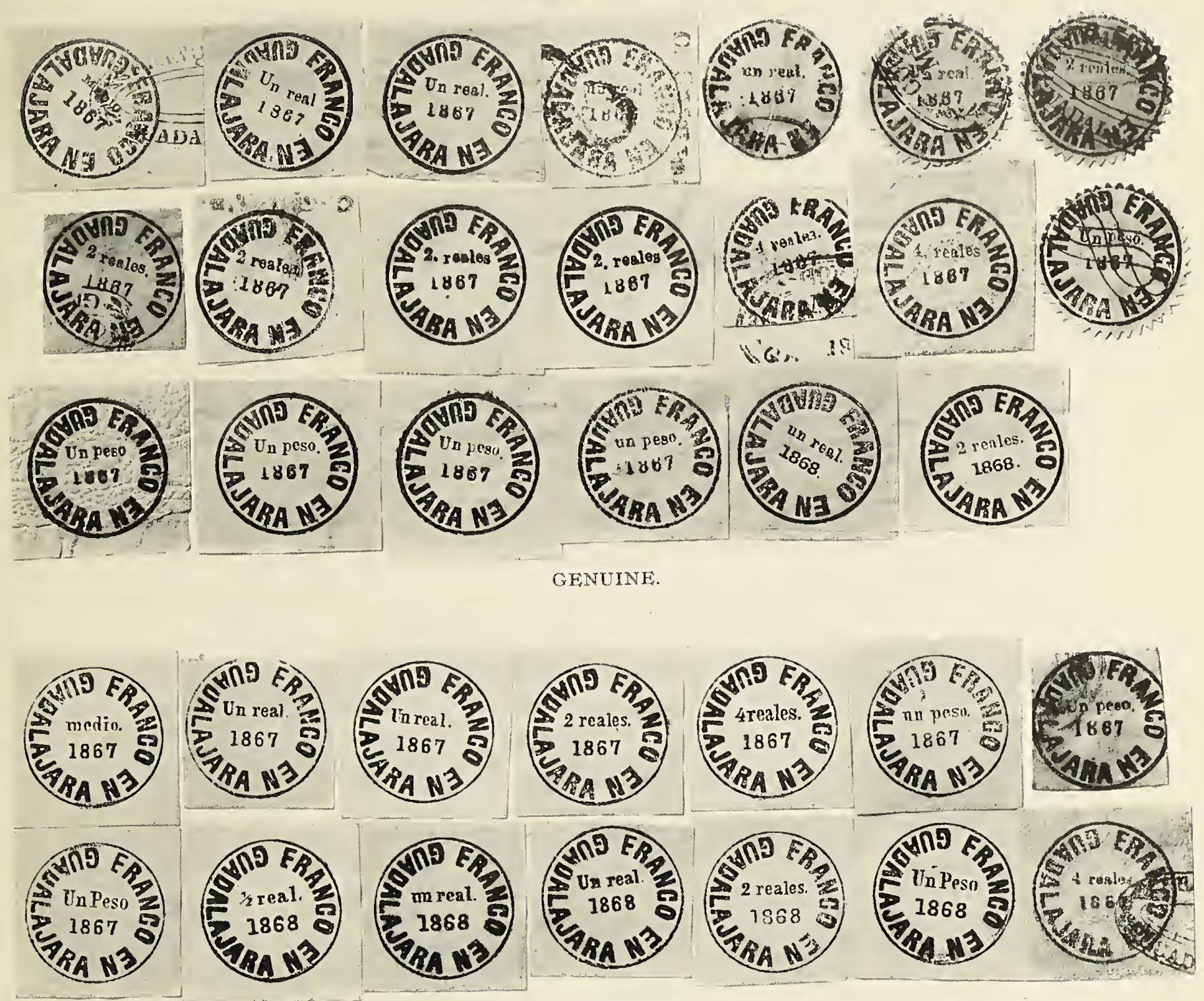

COUNTERFEITS.

CAMPECHE.

CHIAPAS.

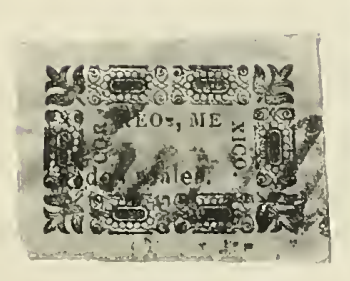

GENUINE.

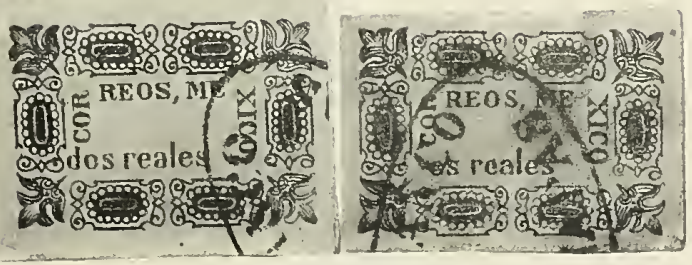

COUNTERFEITS.

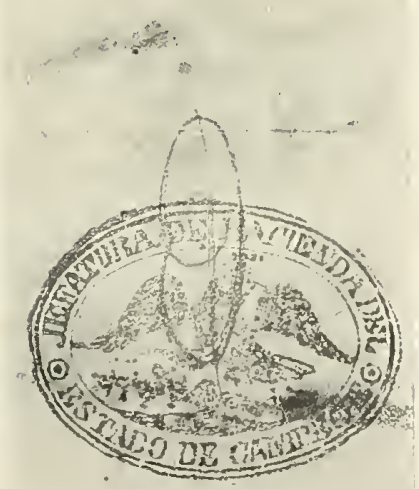



$2^{\circ}$ In the genuine there is, at eaci sile of the label cuntaining the denomination of value, a small double lined circle on the outside of which is a small dot; this dot is missing in the counterfeits.

$3^{\circ}$ The counterfeits are perforated $11 \mathrm{I} / 2$ instead of $\mathrm{I} 2$.

I868 Issue.

There are some pretty good counterfeits of this issue but they can be distinguished from the genuine by the following point :-

In the genuine at the top of the nine frame of the circle containing the head of Juarez, right under the " $x$ " of "Mexico" is a thin horizontal dash, and the first horizontal line of the shading of the circle crosses the inner frame at the top.

I 872 Issue.

The counterfeits of this issue can be told by the following points:-

The lines of the moiré on the back of the genuine are close together, about 47 lines in two centimeters. If held to the light the moiré shows faintly through the back in the genuine, very plainly in the ordinary counterfeits and not at all in to the so-called re-prints. These last are also told by the size, which is a triffe larger $\left(\mathrm{I}_{9} \mathrm{I} / 2 \times 24 \mathrm{~mm}\right.$. instead of $\left.19 \mathrm{~T} / 2 \mathrm{x} / 223 \mathrm{~mm}\right)$. In the genuine the horizontal lines forming the shading between the inner and outer frame of the oval containing the head of Hidalgo project in several places in the white spaces between the colored dots forming the inner frame.

We know of no good forgeries of any of the other issues with the exception of the Porte de Mar stampe, two counterfeits of which are already described in the American Journal of Philately, Vol. VI. pages 605 and 606. Besides these two there is another counterfeit of the issue of August, I875, with figures of value measuring $7 \mathrm{~mm}$. in height; these, however, can be easily identified, as the thin frame which ought to surround the figure of value is missing.

Of the provisional issues of Guadalajara we illustrate on the plate accompanying this number the so-called reprints or counterfeits partly made from the original die, the inscription "Franco en Guadalajara" being from the original die, while the value and date were of a type different from the genuine. Besides comparison the following rules can be depended upon:-
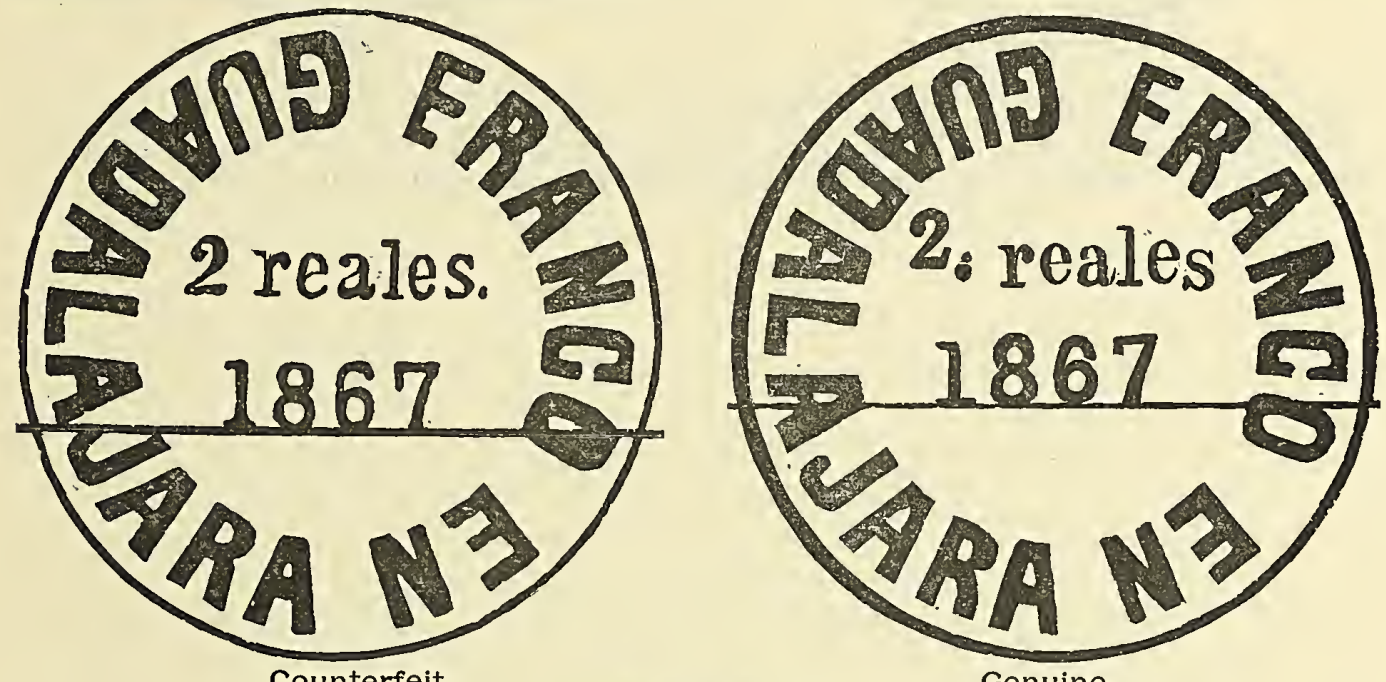

Counterfeit.

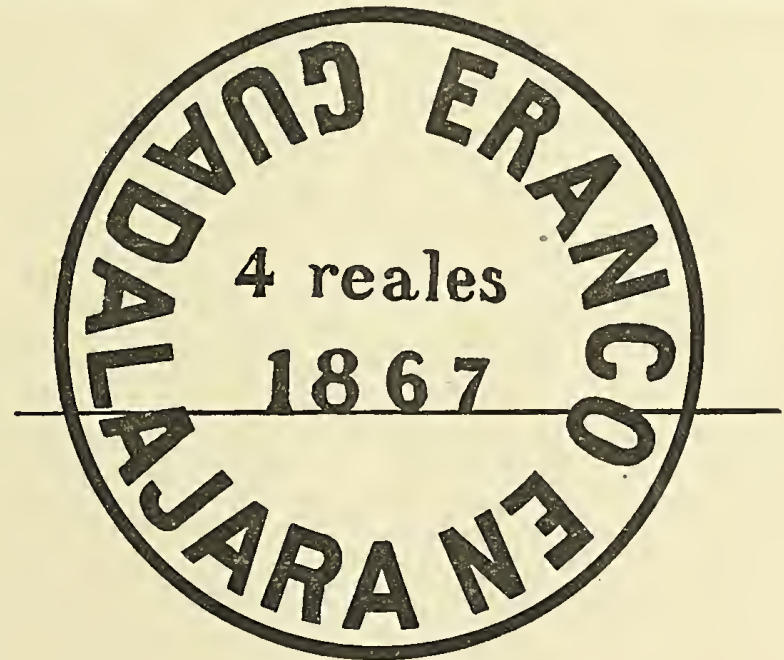

Counterfeit. 
If on a genuine stamp a horizontal line be drawn below the date, 1867 , touching both the $I$ and 7 at bottom and if this line be continued to the right and left it will, as shown per illustration, touch the left side of the "O" of "Franco" at the right and will pass through the centre of the third "A" of "Guadalajara" at the left. If the line be drawn under 1868 , it will pass through the centre of both the " $\mathrm{A}$ " and " $\mathrm{O}$ ". Of all the genuine Guadalajaras we have seen only one specimen (No. II4) which deviated from this rule and we have never seen a counterfeit to which this test was applied without immediately showing its nature.
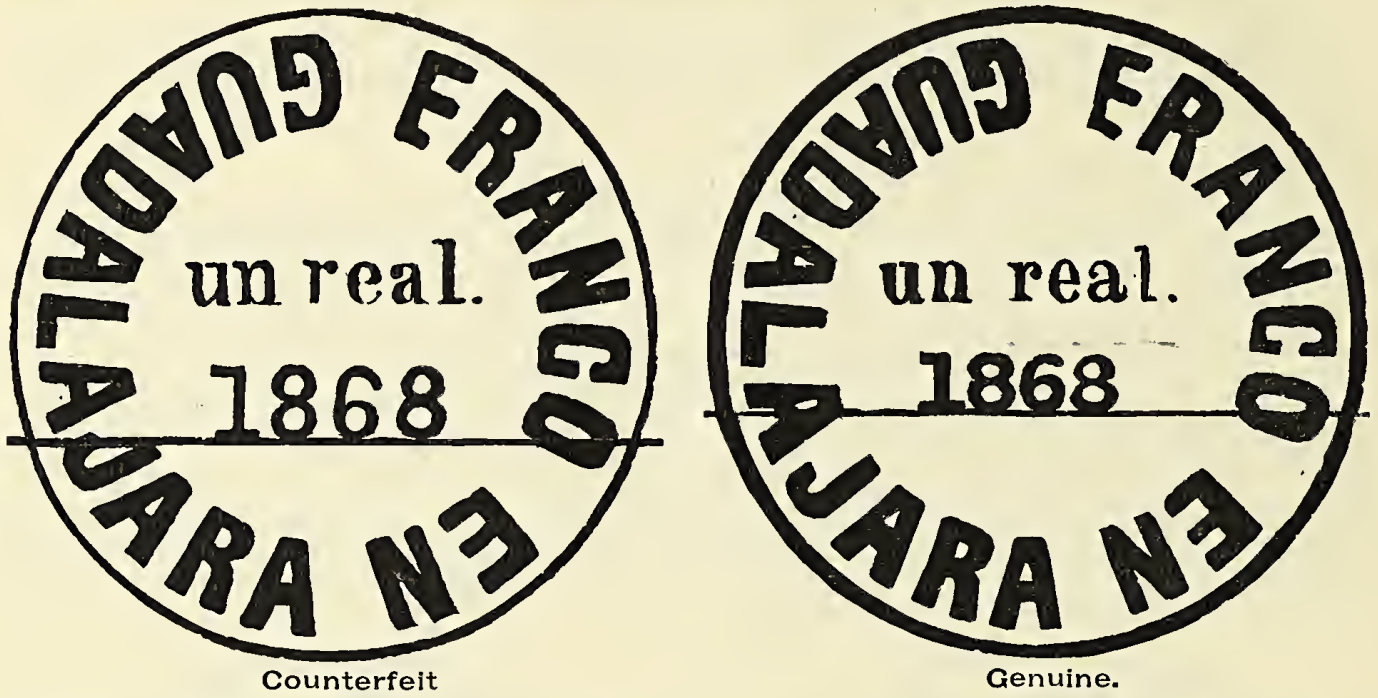

Genuine Guadalajara with forged perforations are plentiful. These are generally easily told by the number of perforations which is 42 on the genuine.

We illustrate also two types of counterfeit Chiapas which for years had been accepted as genuine; their size $30 \times 201 / 2 \mathrm{~mm}$ is sufficient to distingush them from the genuine which measures only $25 \times 17 \mathrm{~mm}$. 



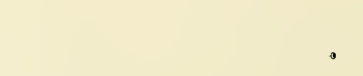





\section{A D D E N A.}

ADHESIVE STAMPS.

I894.

Same type and impression as preceding issue; white wove paper, watermarked CORREOS EUM in every horizontal row of ten stamps.

Perforated 6.

$2128 \quad$ Ic green

$2129 \quad 5 \mathrm{C}$ blue

Varieties :

a. Perforated 6 at top and bottom, perforated $\mathrm{I} 2$ at sides.

2I $30 \quad 5 \mathrm{c}$ blue

b. Perforated 6 at top, bottom and left side, perforated $\mathrm{I} 2$ at right side.

2I3r 5c blue

c. Perforated 6 at top, bottom and right side, perforated $\mathrm{I} 2$ at left side.

$21325 \mathrm{c}$ blue

1895.

Same type and impression as preceding issue.

I. White laid paper, watermarked coRREOS EUM.

Perforated $\mathbf{I 2 .}$

2133 I 2c scarlet

Variety : top or bottom row unwatermarked

2134 I 2c scarlet

II. White laid paper, unwatermarked.

Perforated I2.

2135 2c carmine

$2 \mathrm{I} 36$ 3C vermilion

2137 4c "

2138 IOC

April 2d, I 895

Engraved in taille douce on white wove paper, watermarked CORREOS EUM in every horizontal row of ten stamps. Size $20 \times 25 \mathrm{~mm}$. Perforated I 2.

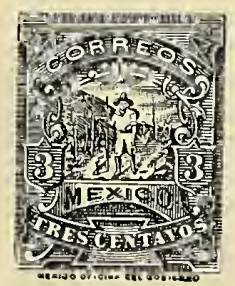

2 I 39 Ic green

2I 40 2C carmine

2 I 4 I 3C yellow brown

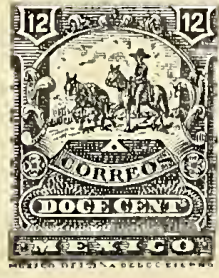

$2142 \quad 4 c$ vermilion

2I43 I 2c olive

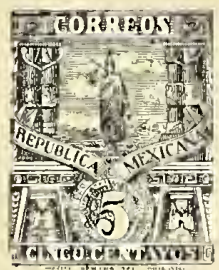

2 I44 5c ultramarine

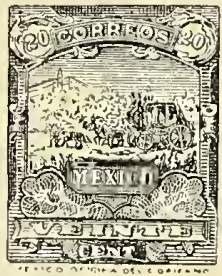

2 I 45 IOc rose lilac

2146 I5 c bright blue green

2 I 47 2Oc brown lilac

$214850 \mathrm{c}$ violet

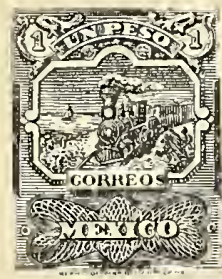

2 I49 Ip brown

2 โ50 5P rose

2I I rop deep blue 
OFFICIAL STAMPS.

April 1894 .

Same type as preceding issue, engraved on white laid paper, watermarked CORREOS EUM in every horizontal row of ten stamps.

$2^{\circ}$ Perforated 6.

2810 blue

Varieties :

a. Last horizontal row unwatermarked.

b. .Perforated $6 \times 12$ irregularly.

2812 blue

c. Perforated 6 and imperforate.

281 3 blue

d. Perforated 12 and imperforate.

2814 blue

The following combinations of perforations exist.

$\begin{array}{cccc}\text { Top. } & \text { Botton. } & \text { Right. } & \text { Left. } \\ \text { Imp. } & \text { Imp. } & \text { Imp. } & 6 \\ \text { Imp. } & 6 & 6 & \text { Imp. } \\ \text { Imp. } & 6 & \text { Imp. } & 6 \\ 6 & \text { Imp. } & 6 & \text { Imp. } \\ 6 & \text { Imp. } & \text { Imp. } & 6 \\ \text { Imp. } & \text { I2 } & \text { I2 } & 6 \\ \text { Imp. } & 6 & \text { Imp. } & \text { I2 } \\ \text { Imp. } & \text { I2 } & 6 & 6 \\ \text { Imp. } & 6 & 6 & 6 \\ \text { Imp. } & \text { I2 } & \text { Imp. } & 6 \\ \text { I2 } & \text { I2 } & \text { Imp. } & 6 \\ \text { I2 } & \text { Imp. } & \text { Imp. } & 6 \\ \text { I2 } & \text { Imp. } & \text { I2 } & 6 \\ \text { I2 } & \text { I2 } & 6 & \text { I2 } \\ \text { I2 } & \text { I2 } & 6 & 6 \\ \text { I2 } & 6 & 6 & 6 \\ \text { I2 } & \text { Imp. } & 6 & 6 \\ \text { I2 } & \text { I2 } & \text { I2 } & \text { I2 } \\ 6 & 6 & \text { I2 } & \text { Imp. } \\ 6 & \text { I2 } & 6 & 6 \\ 6 & 6 & 6 & 6 \\ 6 & \text { Imp. } & \text { Imp. } & \text { I2 } \\ 6 & 6 & \text { I2 } & \text { Imp. } \\ 6 & 6 & 6 & \text { Imp. } \\ 6 & 6 & \text { Imp. } & 6 \\ \text { Imp. } & \text { I2x6 } & 6 & 6 \\ 6 & 6 & \text { I2x6 } & \text { Imp. } \\ 6 & \text { I 2x6 } & 6 & 6 \\ \text { Imp. } & \text { I2x6 } & \text { Imp. } & 6 \\ 6 & \text { I2x6 } & 6 & \text { Imp. } \\ \text { I2 } & \text { I2 } & \text { I2x6 } & 6 \\ \text { I2 } & \text { I2x6 } & 6 & 6\end{array}$

$I^{\circ}$ Perforated $\mathbf{I}$. 28I I blue

\begin{tabular}{|c|c|c|c|}
\hline $\begin{array}{l}\text { Top. } \\
\text { I } 2 \times 6\end{array}$ & $\begin{array}{l}\text { Bottom. } \\
\text { I } 2\end{array}$ & $\begin{array}{c}\text { Right. } \\
6\end{array}$ & $\begin{array}{l}\text { Left. } \\
6\end{array}$ \\
\hline $12 \times 6$ & 6 & 6 & 6 \\
\hline $12 \times 6$ & $12 \times 6$ & Imp. & 6 \\
\hline $12 \times 6$ & Imp. & Imp. & 6 \\
\hline I $2 \times 6$ & Imp. & 6 & Imp. \\
\hline Imp. & 12 & Imp. & I 2 \\
\hline Imp. & 12 & 12 & Imp. \\
\hline 12 & Imp. & I2 & Imp. \\
\hline 12 & Imp. & Imp. & 12 \\
\hline
\end{tabular}

I 895

Same type as preceding issue, engraved on white wove paper unwatermarked.

Perforated I2.

28I 5 carmine

April, I895.

Same type as adhesive stamps of corresponding issue, surcharged diagonally $0 \mathrm{~F}|\mathrm{C}| \mathrm{AL}$ the surcharge measuring $3 \mathrm{x}$ $161 / 2 \mathrm{~mm}$.

I. Black surcharge.

White laid paper.

Watermarked CORREOS EUM

Perforated 12.

\begin{tabular}{|c|c|c|c|}
\hline 2816 & c gree & bla & rcll \\
\hline 2817 & 2c carmine & “ & \\
\hline $28 \mathrm{I} 8$ & 3c yellow brown & " & “، \\
\hline 28 I9 & $4 \mathrm{c}$ vermilion & “ & “ \\
\hline 2820 & 5c ultramarine & “ & ، \\
\hline 2821 & Ioc rose lilac & “" & “ \\
\hline 2822 & rac olive & “ & “" \\
\hline 2823 & $\mathrm{I}_{5} \mathrm{c}$ bright blue green & “ & " \\
\hline 2824 & $20 \mathrm{c}$ brown lilac & " & " \\
\hline 2825 & $50 c$ violet & “ & “، \\
\hline 2826 & Ip brown & “ & “ \\
\hline 282 & $5 p$ rose & “" & “ \\
\hline 2 & Iop deep blue & “ & 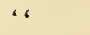 \\
\hline
\end{tabular}

II. Red surcharge.

White laid paper.

Watermarked CORREOS EUM.

Perforated I 2

\begin{tabular}{|c|c|c|c|}
\hline 829 & Ic green, & red & surcharge \\
\hline 2830 & 2c carmine & & \\
\hline 2831 & 3c yellow brown & “" & “ \\
\hline 283 & 4c vermilion & “" & - \\
\hline 2833 & 5c ultramarine & “" & \\
\hline 2834 & Ioc rose Jilac & “" & ' \\
\hline 283 & I2c olive & “ & ، \\
\hline 283 & I5 $\mathrm{C}$ bright blue green & “. & “. \\
\hline 233 & $20 \mathrm{c}$ brown lilac & “" & “ \\
\hline 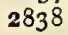 & 5oc violet & ". & “" \\
\hline
\end{tabular}

\section{ENVELOPES}

October $\mathrm{I}_{3}$ th, $\mathbf{1} 894$.

Stamps of same type as adhesive stamps of corresponding issue, embossed in upper right corner on white wove paper.

Size $152 \times 85 \mathrm{~mm}$.

$I^{0}$ Laid paper.

$\begin{array}{cc}3204 & 4 \mathrm{c} \text { vermilion } \\ 3205 & \text { Ioc } \text { " } \\ 2^{\circ} & \text { Wove paper. } \\ 3206 & \text { 5c ultramarine } \\ 3207 & \text { Ioc vermilion }\end{array}$

Stamps of same type as corresponding values of the adhesive stamps of same issue, embossed in upper right corner on white wove paper, un watermarked; Coat of Arms printed in rose in upper left corner.

Size $149 \times 90 \mathrm{~mm}$.
3208
$4 \mathrm{c}$ vermilion and rose
$32095 \mathrm{c}$ ultramarine and rose
$3210 \quad$ IOc rose lilac and rose

April 2d, I895. 



\section{WRAPPERS.}

April 25, 1895 .

Inscriptions typograph 2 d in color and stamp engraved in color on manila paper; flap at the right, gummed, truncated end. Both values have the word FAJILLLA spelled with three L's.

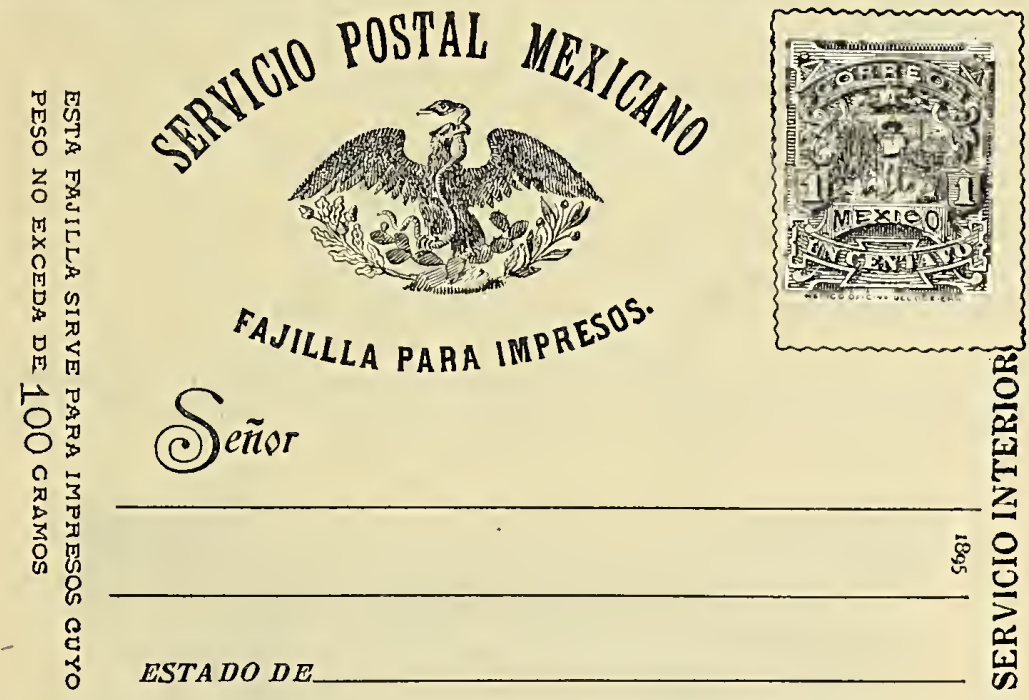

Size $250 \times 82 \mathrm{~mm}$.

3609 Ic green, dark green inscriptions

36I0 Ic green, gray blue "“

36 II 2c carmine, rose

3612 2c carmine, carmine, rose inscriptions Variety: Senor (broken $\mathrm{r}$ )

36I3 2c carmine, rose inscriptions

POSTAL CARDS.

June 1879 .

Lithographed in black or color on cards of various colors; double-lined rectangle for pasting stamp at upper right corner. Size, $140 \times 70 \mathrm{~mm}$.

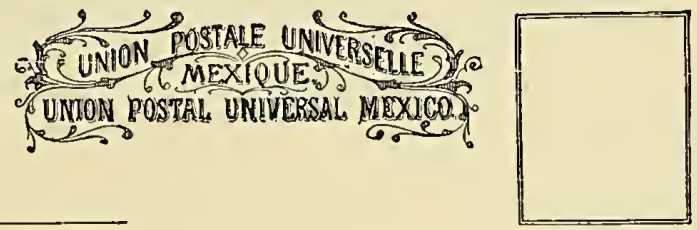

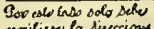

$400 \mathrm{I}$ No value, rose impression on various cards.
a. On greenish blue card.
b. On light green card.
c. On sea green card.
d. On yellow green card.
e. On dark blue card.
f. On pale blue card.
g. On rose lilac card.
h. On pale rose card.
i. On cream rose card.
j. On yellow card.
k. On white card.
1. On gray card.

4002 No value, pink impression, on various cards.

a. On rose card.

b. On yellow card.

4003 No value, blue impression, on various cards.
a. On yellow card.
b. On salmon card.
c. On rose card. 
d. On reddish buff card.

e. On green card.

f. On bluish slate card.

g. On pale slate card.

h. On sea green card.

i. On lilac card.

j. On purple card.

k. On gray card.

4004 No value, black impression, on various cards.

a. On violet card.

b. On brick card.

c. On gray card.

d. On green card.

e. On brown card.

f. On buff card.

4005 No value, buff impression, on straw card.

These cards were used by the addition of an adhesive stamp of the current issue.

I882.

Lithographed in blue on various colored cards; stamps bearing the head of President Juarez, embossed in brown at the right; surcharged in black in upper left corner with abbreviated date and number, and sometimes name of postal district, size $137 \times 92 \mathrm{~mm}$.

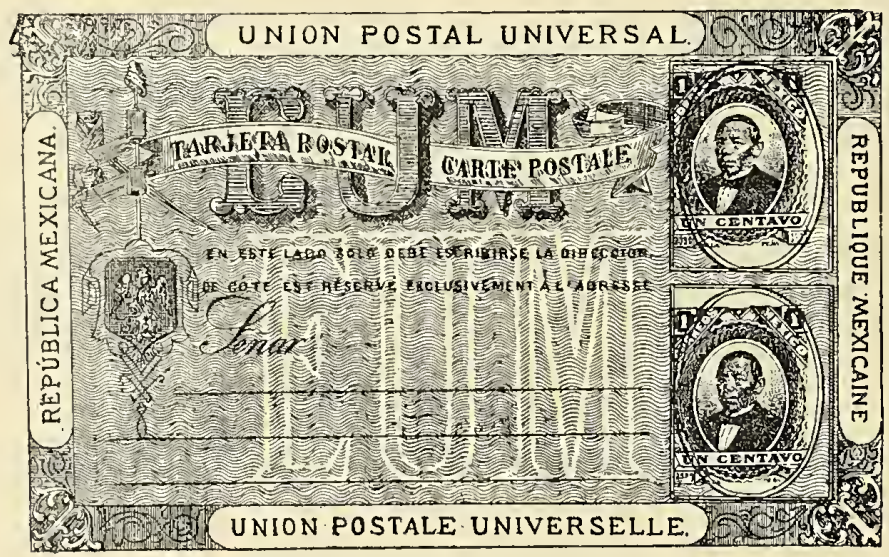

4006 IxIc brown, blue impression, on various cards.

a. On lilac card.

b. On pale yellow card.

c. On rose card

d. On greenish blue card.

e. On biuish green card.

f. Not dated, on lilac card.

g. Not dated and without stamps, on lilac card.

The following are the dates and district numbers known on these cards :

$$
\begin{aligned}
& 382 \text { (Vera Cruz). } \\
& 682 \text { (Tula). } \\
& 882 \text { (Tuxpan). } \\
& 2682 \text { (Merida). } \\
& 2682 \text { Merida. } \\
& 2782 \text { (Mazatlan). } \\
& 3982 \text { (Chihuahua). } \\
& 4782 \text { (C. Victoria). } \\
& 5382 \text { (Acapulco). } \\
& 5482 \text { (Mexico). }
\end{aligned}
$$

March, I882.

Same type, impression etc., as preceding issue, but stamp in upper rectangle is of the $2 c$ denomination. $40072 \times I c$, lilac and brown, pink impression, on various cards.

a. On pale yellow card.

b. On greenish blue card.

c. On rose card.

d. On lilac card.

e. Not dated, Ic stamp is printed in lilac in upper rectangle, and the $2 \mathrm{c}$ in brown in lower rectangle on pale yellow card.

f. No stamp and not dated, carmine impression, on pale yellow card.

g. No stamp and not dated, carmine impression, on pale yellow paper.

The following are the district numbers known on these cards : 4782 (C. Victoria). 5482 (Mexico). 


July, 1882.

Card same as preceding issue, with stamp of new type; surcharged with abbreviated date, number and sometimes name of postal district. Size I $37 \times 9 \mathrm{r} \mathrm{mm}$.

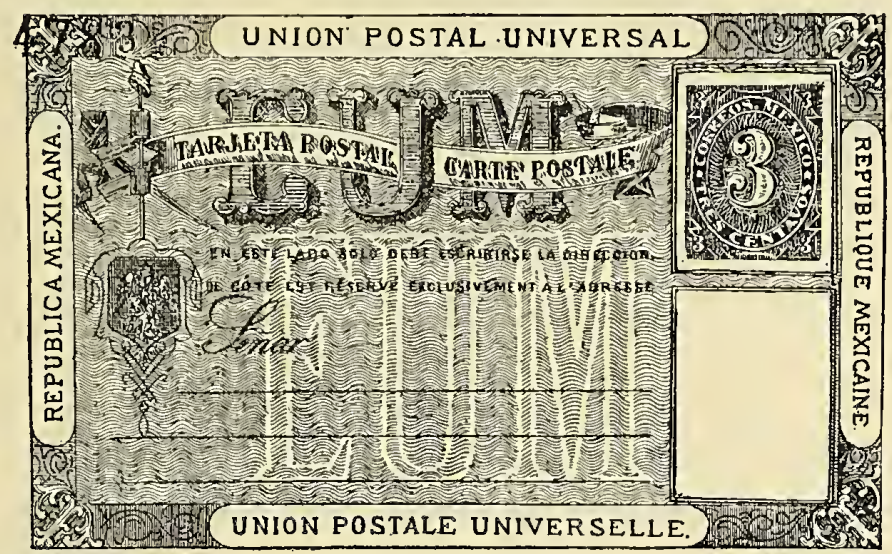

4003 3c carmine, blue impression on buff card.
a. With date.
b. Not dated.
c. No date and no stamp.

$40093 \times 2 \mathrm{c}$ carmine and green, blue impression on buff card.

a. With date.

b. Without date.

The following are the dates and numbers of postal districts, known on these cards.

$$
\begin{aligned}
& 1782 \text { (S. L. Potosi). } \\
& 2582 \text { Monterey. } \\
& 2582 \text { (Monterey), } \\
& 2682 \text { (Merida). } \\
& 2882 \text { Matamoros. } \\
& 2882 \text { (Matamoros). } \\
& 3782 \text { (Guadalajara). } \\
& 3982 \text { (Chihuahua). } \\
& 5482 \text { (Mexico). } \\
& 283 \text { (Zacatecas). } \\
& \text { I883 (Saltillo). } \\
& \text { I883 Mazatlan. } \\
& 2583 \text { (Monterey). } \\
& 5383 \text { (Acapulco). } \\
& 5483 \text { (Mexico). }
\end{aligned}
$$

March, I884.

Lithographed in carmine on buff card, with stamp embossed in green in upper right corner. Sizo I $40 \times 93 \mathrm{~mm}$

Beginning with this $i$;sue the cards are no longer surcharged with date and ostal district number.

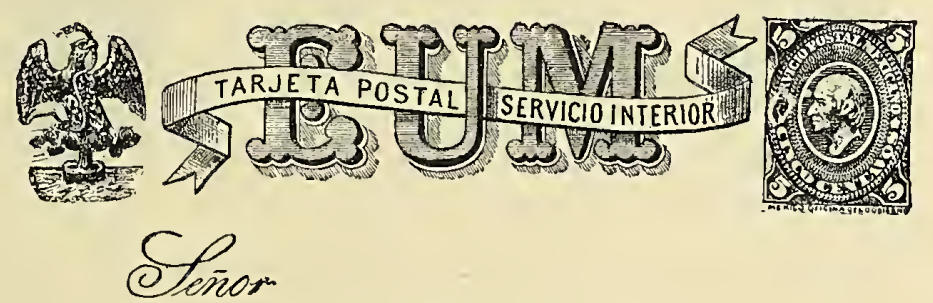

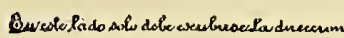

40 ro $5 \mathrm{c}$ green, carmine impression on buff card. 
Lithographed in black on buff card, without stamp.

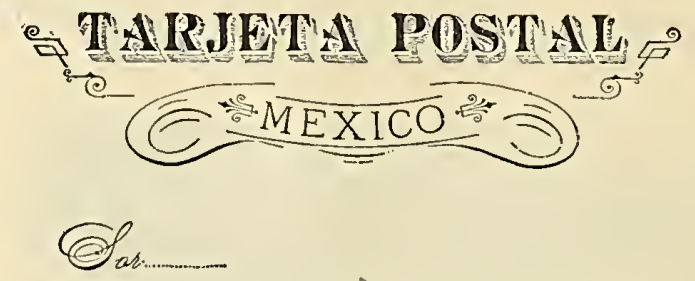

Estado de

40 I No value, black impression, on buff card.

June, I884.

Litho graphe 1 in black, on buff card, stamp embossed in green in upper right corner. Size $143 \times 90 \mathrm{~mm}$.

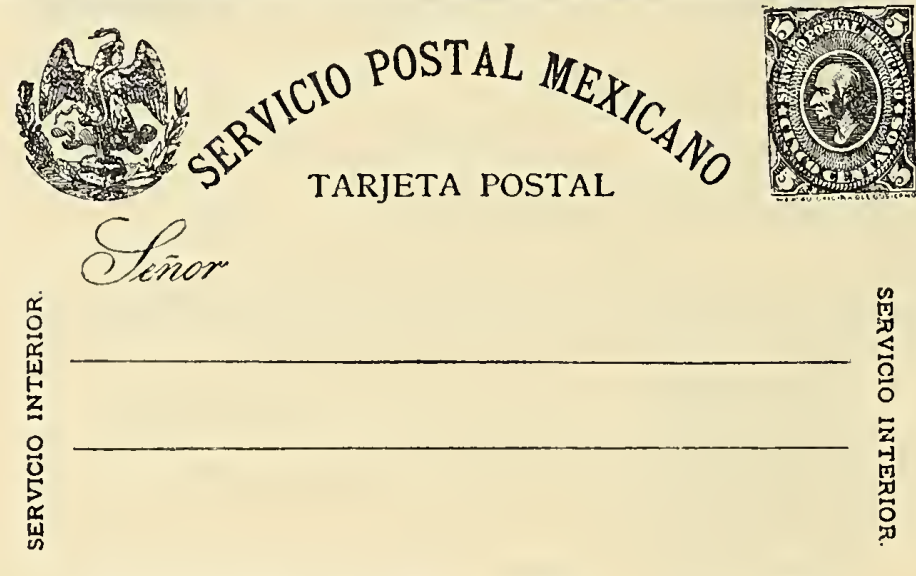

OI2 5c green, black impression on buff card.

a. The two ruled lines are II $\mathrm{mm}$. apart ; the first ruled line is II $71 / 2 \mathrm{~mm}$. long.

b. The two ruled lines are I $\mathrm{mm}$. apart ; the first ruled line is $\mathrm{I} 2 \mathrm{I} 1 / 2 \mathrm{~mm}$. long; period after Direccion

c. Same as b, but without period after Direccion.

d. The two ruled lines are $13 \mathrm{~mm}$. apart.

e. Stamp printed horizontally.

f. Stamp printed upside down in lower left corner.

October, I884.

Lithographed in black on buff card; stamp embossed in green in upper right corner. Size 14 f $93 \mathrm{~mm}$.

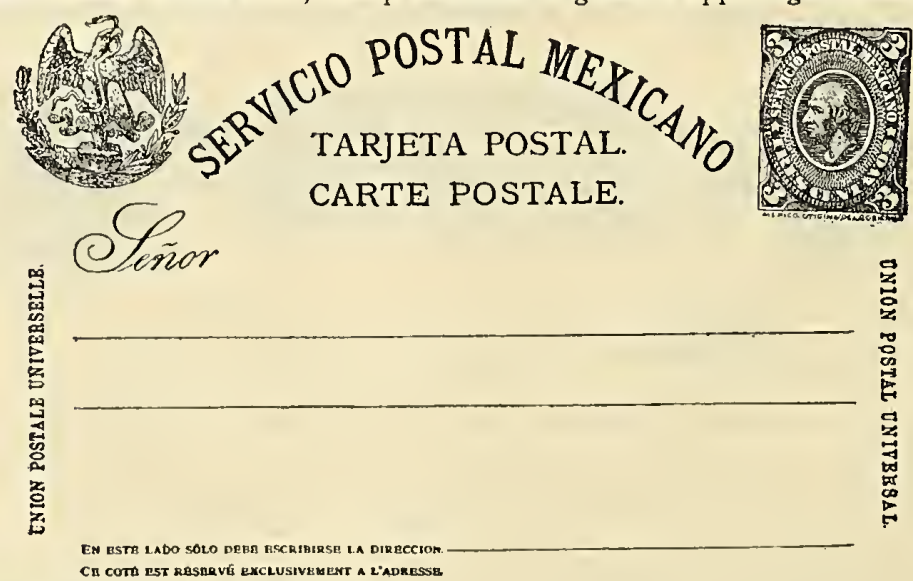

4 OI3 3c green, black impression on buff card.

a. No accent over last $\mathrm{E}$ of ESCRIBIRSE.

b. Accent over last E of ESCRIBIRSE. 


July, 1885 .

Lithographed in black on buff cards; stamp embossed in green in upper right corner. Size $143 \times 90 \mathrm{~mm}$.

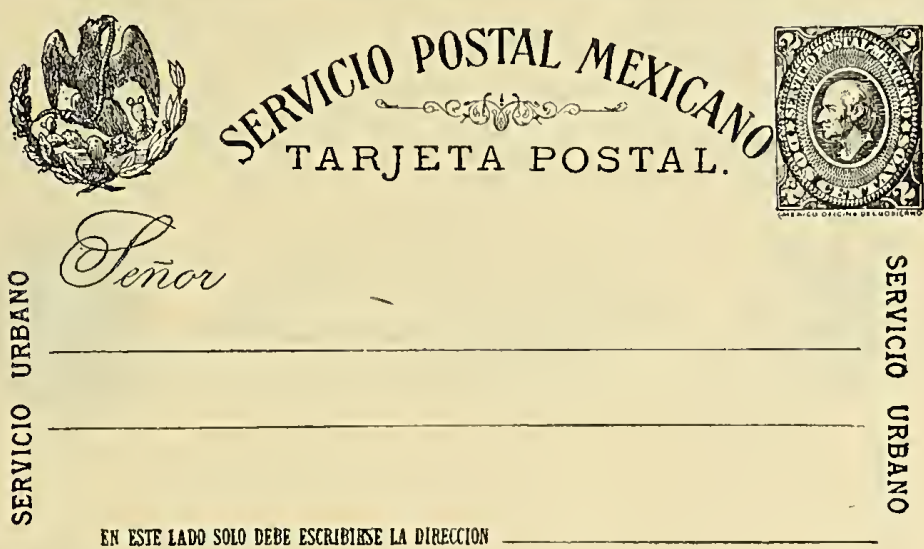

40142 2c green, black impression on buff card.

a. Stamp straight.

b. Stamp upside down.

c. Stamp slanting to the left.

d. Without stamp.

Lithographed in black on buff card; stamp embossed in color in upper right corner. Size $143 \times 93 \mathrm{~mm}$.

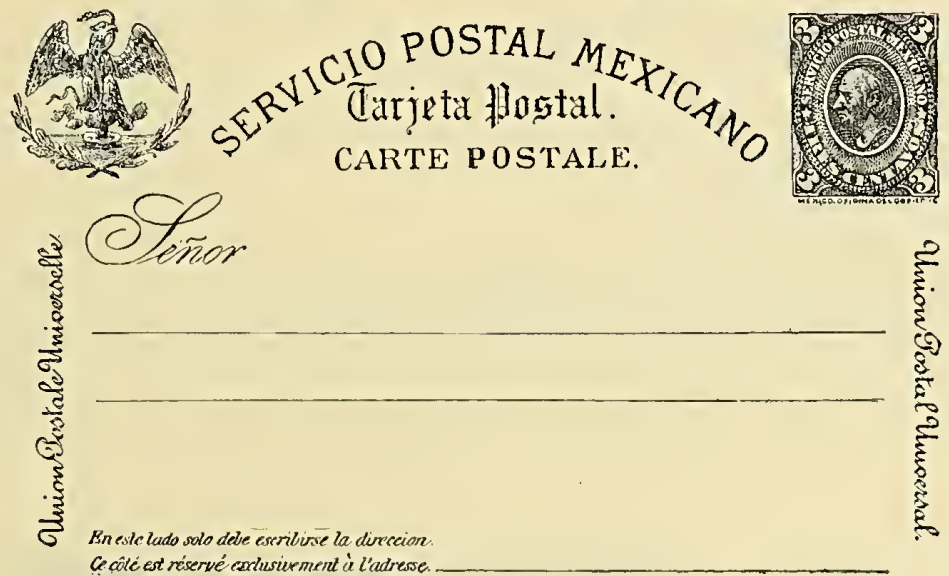

40152 carmine, black impression on buff card.

a. Stamp straight.

b. Stamp upside down.

$40163 \mathrm{c}$ red brown, black impression on buff card.

Lithographed in black on buff card; stamp embossed in blue in upper right corner. Size $193 \times 93 \mathrm{~mm}$.

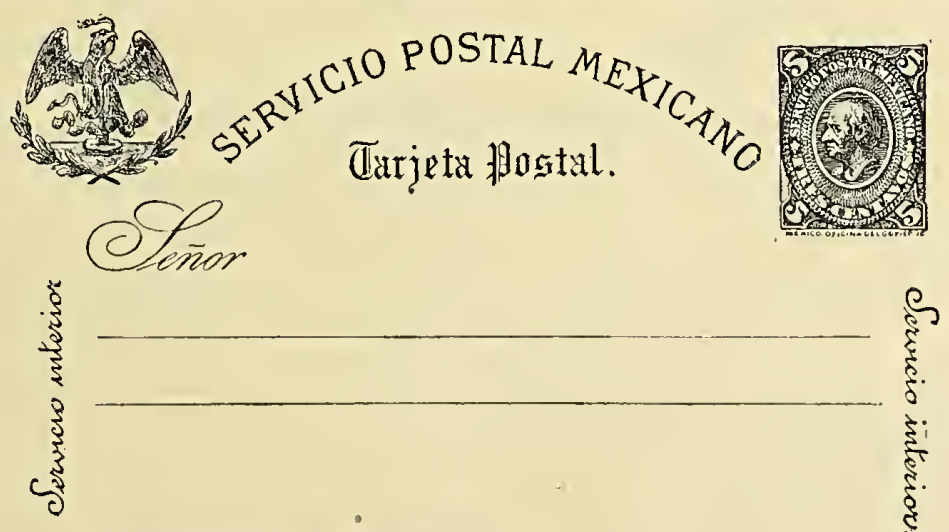

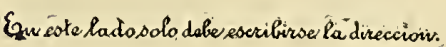


$40175 \mathrm{c}$ blue, black impression on buff card.

a. Pale blue stamp.

b. Dark blue stamp.

c. Dark blue stamp, inverted.

d. Dark blue stamp, inverted in lower left corner.

e. POTAL instead of POSTAL.

January, 1886.

Lithographed in black on buff card ; stamp embossed in upper right corner. Cards folded at top and reply is on the fourth side. Size $143 \times 95 \mathrm{~mm}$.

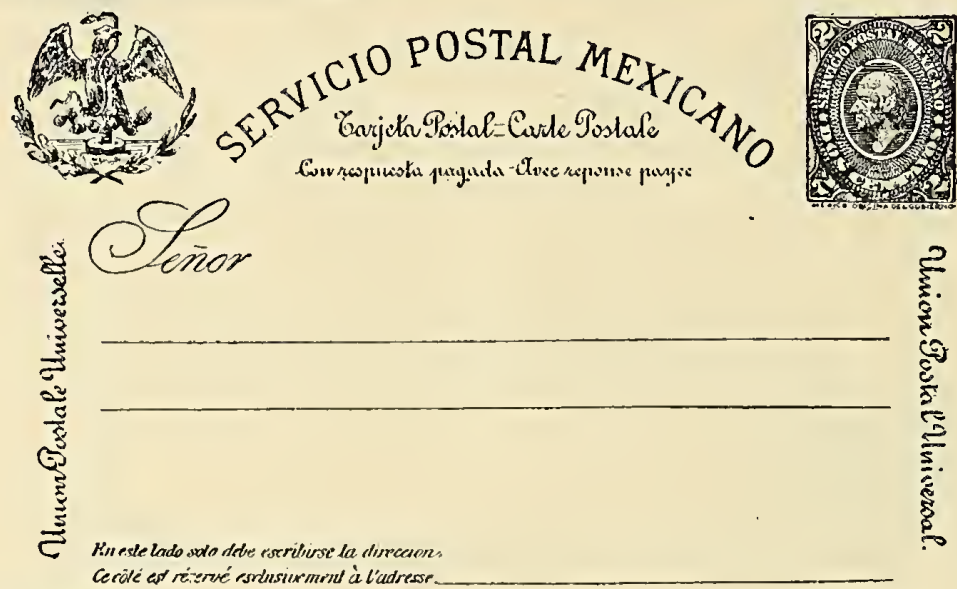

4018 2c carmine, blackimpression on buff card.

$40193 \times 3 \mathrm{c}$ red brown, black impression on buff card.

Same as No. 4 or 4 .

$40202 \times 2 c$ carmine, black impression on buff card.

$40215 \times 5 \mathrm{c}$ blue, black impression on buff card.

a. Servicio Interior at sides.

b. Error, Servicio Urbano at sides.

c. Double impression of stamp.

d. Stamp of first card impres:ed half on reply card.

e. On the first card the vertical inscription at left is missing; at right Servicio Interior. On the second card the vertical inscription at right is missing.

f. on the first card the vertical inscription at left is missing; at right Servicio Urbano.

May, 1886. I $40 \times 93 \mathrm{~mm}$.

Lithographed in black or color on buff card, stamp impressed in blue in upper right corner. Size
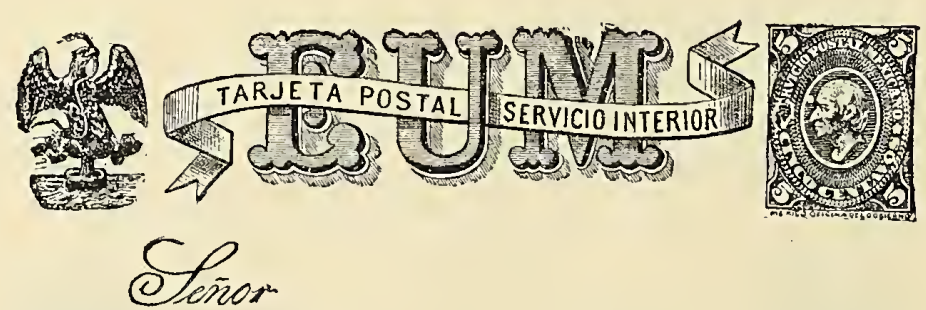

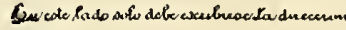

$40225 \mathrm{c}$ blue, black or colored impression on buff card.

a. Black impression,

b. Blue impression.

c. Light brown impression.

d. Dark brown impression.

e. Red impression.

f. Without stamp, green impression. 


Lithographed on buff card, without stamp. Size I $40 \times 93 \mathrm{~mm}$.

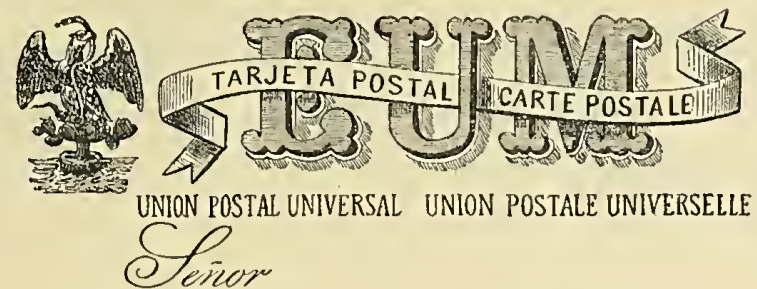

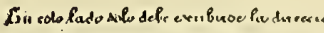

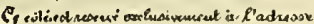

4023 No value, colored impression on buff card.

a. Blue impression.

b. Dark brown impression.

Nos. 4022 and 4023 are supposed to be speculative issues.

November, I 886.

Lithographed in black on buff card; stamp impressed in upper right corner. Size I43x93 mm.

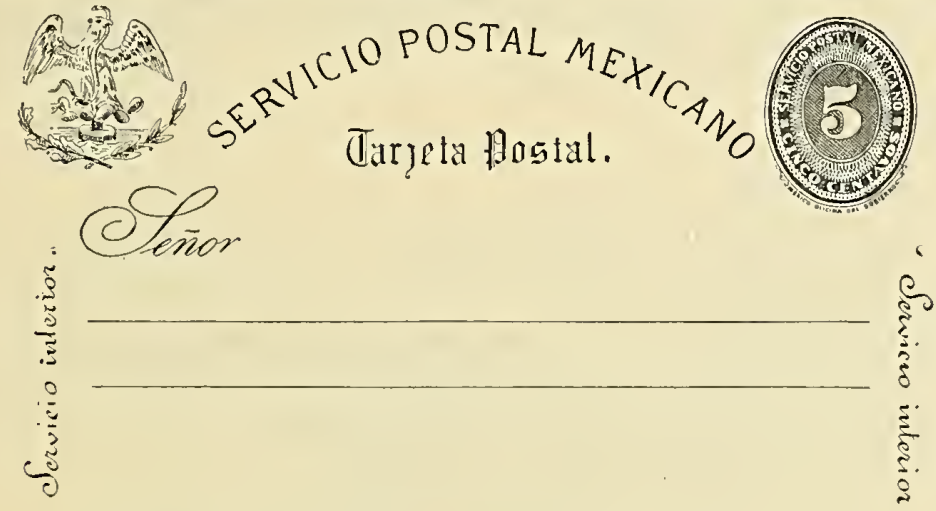

Ens ste lada sulo debe escriburos la direccion

$40245 \mathrm{c}$ blue, black impression on buff card.

a. $T$ of POSTAL in first line over right stroke of $P$ of POSTAL in second line.

b. T of POSTAL in first line over left stroke of P of POSTAL in second line.

December, r886.

Typographed in black on buff card ; stamp embossed in color in upper right corner. The $2 \mathrm{c}$ and $3 \mathrm{c}$ cards are inscribed at right UNION POSTALE UNIVERSELLE, at the left UNION POSTAL UNIVERSAL, measuring respectively 65 and $55 \mathrm{~mm}$.; and a single dash between POSTAL and CARD. The $5 \mathrm{c}$ card has at right and left the inscriptions Servicio Interior. Size I $40 \times 9 \mathrm{Im}$.

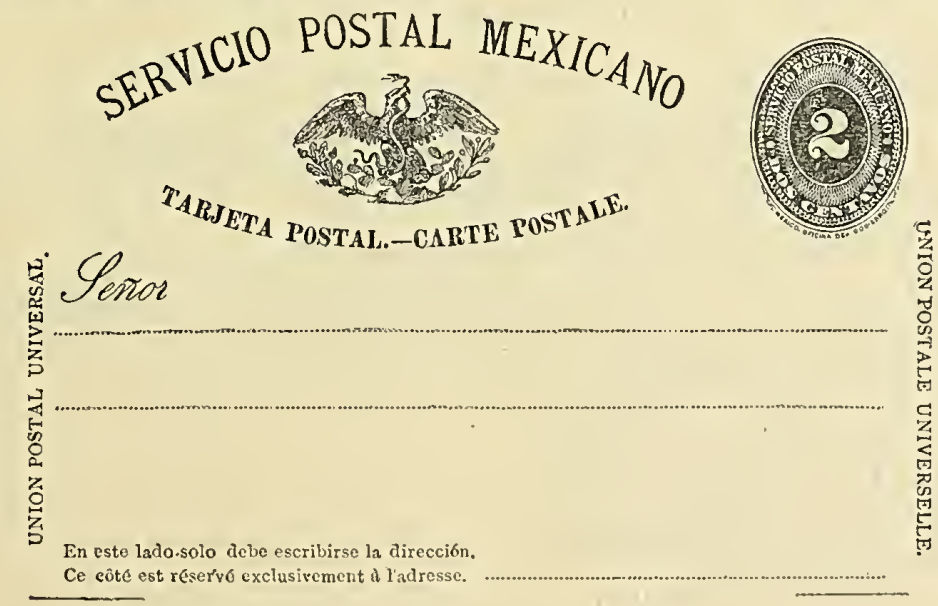


I. $S$ of SENOR is open.

40252 carmine, black impression on buff card.

a. The dotted lines of address begin before $A$ of POSTAL and $V$ of UNIVERSAL at left, and $S T$ and $U$ at right.

b. The two lines for address begin before $\mathrm{A}$ and between $\mathrm{I}$ and $\mathrm{V}$ at the left, and before $\mathrm{S}$ and $\mathrm{V}$ at the right.

c. UNIVERSAL at the left is not punctuated.

4026 3c carmine, black impression on buff card.

a. Same as 4025 a.

b. Same as 4025 b.

c. Same as $4025 \mathrm{c}$

$40275 \mathrm{c}$ blue, black impression on buff card.

a. No period after MEXICANO; last $\mathrm{R}$ of Interior at left, and of Servicio at right have round ends; all the others have straight ends.

b. No period after MEXICANO; the first $\mathrm{R}$ of Interior at left and both $\mathrm{Rs}$ in Interior at right have round ends; all the others have straight ends.

c. No period after MEXICANO; R of Servicio and last R of Interior at left and right have round ends ; all the others have straight ends.

d. Period after mexicnNo; $\mathrm{R}$ of Servicio at left and last $\mathrm{R}$ of Interior at left and right have round ends.

e. Period after MEXICANO; EN in lower left corner is missing; comma after Interior at right; frst $\mathrm{R}$ of Interior at left and right have round ends.

f. TABJETA instead of TARJETA.

g. No period after Interior at left.

July, 1887 .

Same as Nos. 4025 and 4026 with three dashes between POSTAL and CARD. The inscription at left measures $4 \mathrm{r} / 2$ and at right $48 \mathrm{~mm}$.

$40282 \mathrm{c}$ carmine, black impression on.buff card.

a. ETA of TARJETA and T of POSTAL are in smaller type than the other letters.

b. T of POSTAL under arms slightly larger than other letters. Mexicano below the line.

c. POSIAL at left instead of POSTAL.

4029 3c lilac, black impression on buff card.

a. Same as 4028 a.

b. Same as $4028 \mathrm{~b}$.

c. Same as $4028 \mathrm{c}$.

d. Stamp inverted in lower left corner.

Same as Nos. 4028 and 4029 , with only one dash between POSTAL and CARD. Inscription at left measures $43 \mathrm{r} / 2 \mathrm{~mm}$., and at right $50 \mathrm{~mm}$.

$40302 c$ carmine, black impression on buff card.

a. Period after meXICANo.

b. No period after MEXICANO; A in LA in instructions is to the left of $\mathrm{A}$ in line below.

c. No period after MEXICANO; A of LA is over A in line below. A of POSTAL, under arms smaller than the other letters, and no period after postale.

d. No period after MEXICANO, and no period after UNIVERSAL.

e. Same as d but period after UNIVERSAL below the line.

f. Same as $d$ but period after UNIVERSAL $1 / 4 \mathrm{~mm}$. above the line.

g. Same as d but period after UNIVERSAL Imm. above the line.

h. Same as d but stamp inverted in upper right corner.

i. R of SENOR under T of TARJETA instead of under R.

403 I 3c lilac, black impression on buff card,

a. Same as 4030 a.

b. Same as $4030 \mathrm{~b}$.

c. Same as $4030 \mathrm{c}$.

d. Same as 4030 d.

e. Same as 4030 e.

f. Same as $4030 \mathrm{f}$.

g. Same as $4030 \mathrm{~g}$.

i. Same as e, but U of Universal at right below the line.

II. $S$ of SENOR closed.

Servicio Interior at right and left sides measures respectively $321 / 2$ and $32 \mathrm{~mm}$.

$40325 \mathrm{c}$ blue, black impression on buff card.

a. The R of Servicio and last R of Interior at left have round ends; period after POSTAL is above the line. The two lines for address begin before $\mathrm{N}$ and $\mathrm{O}$ at left and $\mathrm{I}$ and $\mathrm{N}$ at right.

$\mathrm{b}$. The two lines for address begin before $\mathrm{N}$ and $\mathrm{O}$ at left and $\mathrm{I}$ and $\mathrm{T}$ at right.

c. Same as a, but with comma after direccion.

d. POTAL in first line instead of POSTAL; otherwise same as a.

October, 1887 .

Same as Nos. 4030 and $403 \mathrm{I}$, but on the $2 \mathrm{c}$ card the inscriptions at left and right measure respectively 55 and $65 \mathrm{~mm}$., and on the $3 \mathrm{c}$ they measure respectiyely 42 and $47 \mathrm{~mm}$., and on both the letters of the inscriptions at the sides are $21 / 2 \mathrm{~mm}$. high.

$40332 \mathrm{c}$ carmine, black impression on buff card.

a. Dotted line correct.

b. $49 \mathrm{~mm}$. of first dotted line missing.

c. $68 \mathrm{~mm}$. of first dotted line missing.

d. CE CO TE instead of CE COTE.

$40343 \mathrm{C}$ vermilion, black impression on buff card.

a. CE CO TE instead of CE COTE.

b. Same as a, with stamp inverted, 


$$
\text { . }
$$



April, $\mathbf{1} 888$.

Same as No. 4032. The inscriptions at left and right measure 38 and $39 \frac{1}{2} \mathrm{~mm}$. respectively, and the letters are $21 / 2 \mathrm{~mm}$. high.

$40355 \mathrm{c}$ blue, black impression on buff card.

a. Instructions in lower left corner measure $573 / 4 \mathrm{~mm}$., POSTAL is punctuated.

b. The instructions measure $561 / 2 \mathrm{~mm}$. and POSTAL is punctuated.

c. Same as a, but POSTAL is not punctuated,

d. Same as a, but stamp is printed horizontally.

e. Same as a, but stamp is printed diagonally.

January, $\mathbf{1 8 8 9 .}$

Same as Nos. 4033 and 4034, but Exclusivemente and Cote without accent on $\mathbf{E .}$

4036 2c carmine, black impression on buff card.

a. The two lines for address begin before $N$ and $P$ at left and $U$ and $R$ at right.

b. The two lines for address begin before $N$ P and $T$ at left and $U$ and $R$ at right.

c. Stamp impressed diagonally.

$40373 \mathrm{c}$ vermilion, black impression on buff card.

a. Same as $4036 \mathrm{a}$, first dotted line is complete. TARJETA measures $671 / 2 \mathrm{~mm}$.

b. Same as a, but $68 \mathrm{~mm}$., of first dotted line missing.

c. Same as $4036 \mathrm{~b}$, but acute accent over o of direccion.

d. Same as c, but CE of CE COTE is missing.

e. Stamp inverted.

Same as 4035 , with inscriptions at sides $401 / 2 \mathrm{~mm}$, long.

$40385 \mathrm{c}$ blue, black impression on buff card.

a. O of SENOR upside down ; grave accent over o of direccion; instructions at bottom $50 \mathrm{~d} / 2 \mathrm{~mm}$. long.
The lines for address begin before $\mathrm{O}$ and $\mathrm{E}$ at left and I and I at right.

b. Same as a; instructions at bottom $59 \mathrm{~mm}$. long.

c. Same as a; acute accent over o of direccion. The two lines for address begin before I and TI at left and or and $\mathrm{R}$ at right.

d. Stamp inverted in lower left corner.

February, 1889 .

Same as 4036 , but TARJETA POSTAL in large thin capitals, COTE without accent on the 0.

4039 2c carmine, black impression on buff card.

The two lines for address begin before $\mathrm{P}$ and $\mathrm{L}$ at the left and $\mathrm{N}$ and $\mathrm{s}$ at right.

August, I889.

Lithographed in color on various colored cards, stamp embossed in upper right corner. Size I39x9omm.

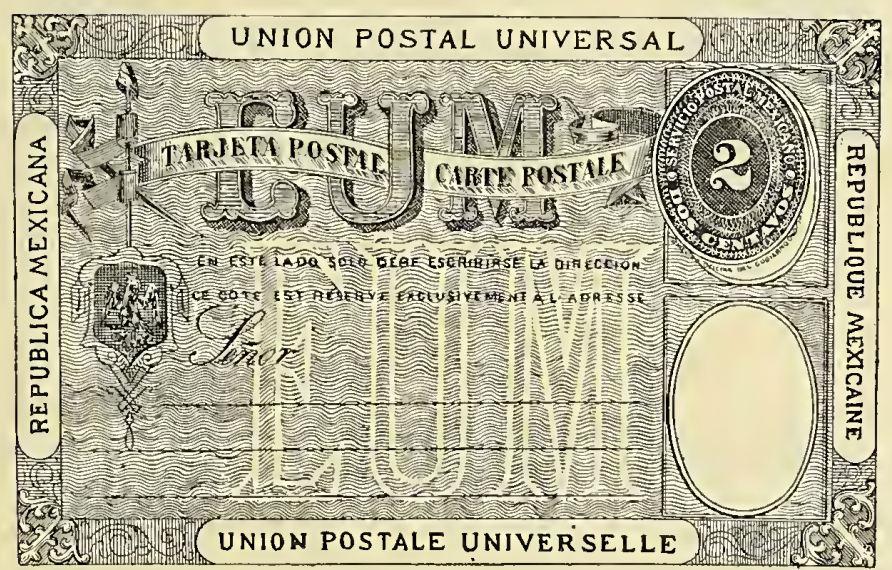

$40402 \mathrm{c}$ carmine, colored impression on various cards.

a. Blue impression on lilac card.

$404 \mathrm{I} 3 \mathrm{C}$ vermilion, colored impression on various cards.

$\begin{array}{ll}\text { a. Blue impression on lilac card. } & \text { b. Rose impression on rose card. }\end{array}$

b. Rose impression on rose card.

Lithographed in blue on buff card. Stamp embossed in upper right corner. Size $139 \times 92 \mathrm{~mm}$.

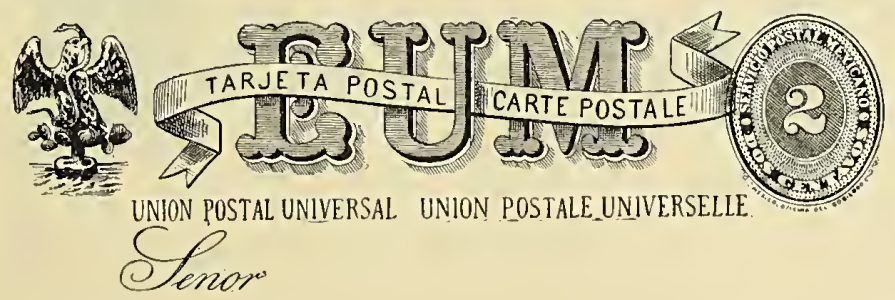


4042 2c carmine, blue impression on buff card.

a. Only one stamp impressed.

b. Two stamps impressed, one under the other, the second one having no color.

4043 3c lilac, blue impression on buff card.

$40443 \mathrm{c}$ vermilion, blue impression on buff card.

January, I89o.

Typographed in color on white card; stamp embossed in upper right corner. Size I4IX9I mm.
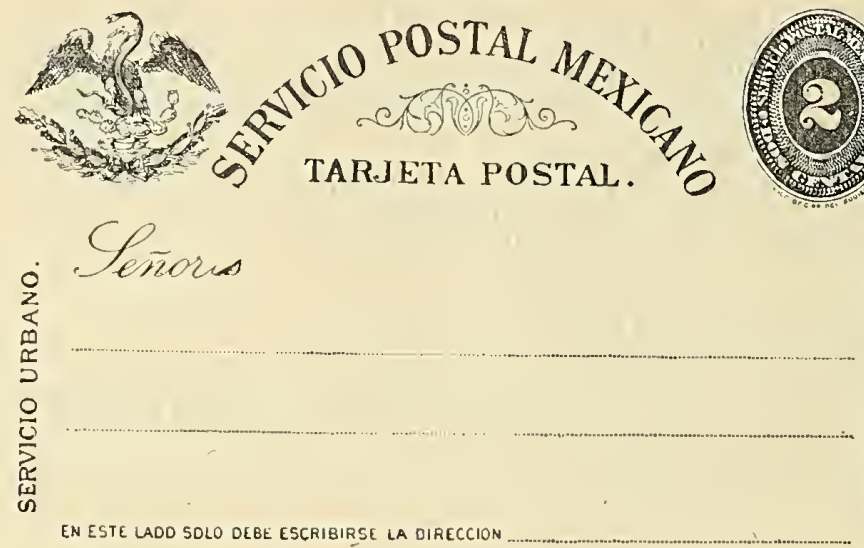

0452 carmine, blue impression on white card.

a. URBANO at right punctuated.

b. URBANO at right not punctuated. .

c. INTERIOR instead of URBANO at sides.

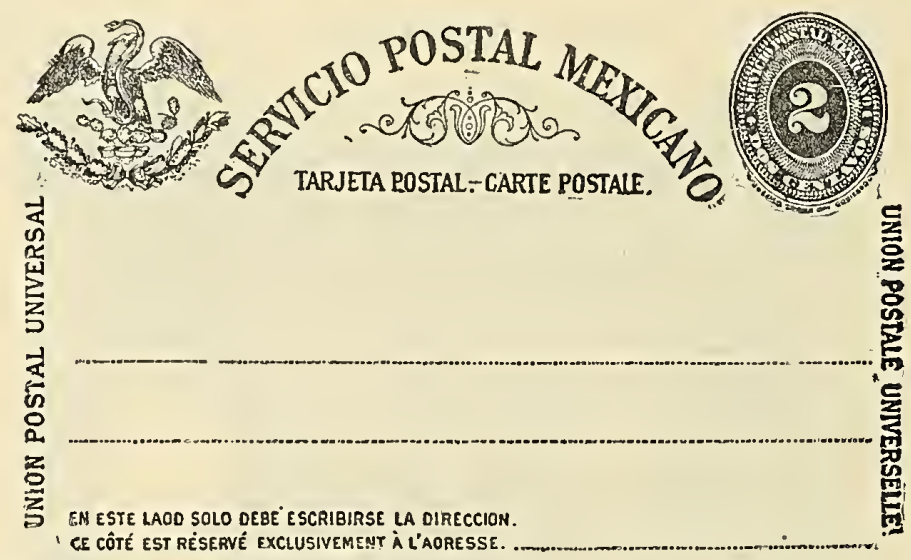

4046 2c carmine, green impression on white card.

a. Yellow green impression.

b. Green impression.

c. Double impression of stamp, one without color.

d. Stamp inverted in lower right corner.

e. Part of stamp embossed in the centre of right side.

$40473 \mathrm{c}$ vermilion, green impression on white card.
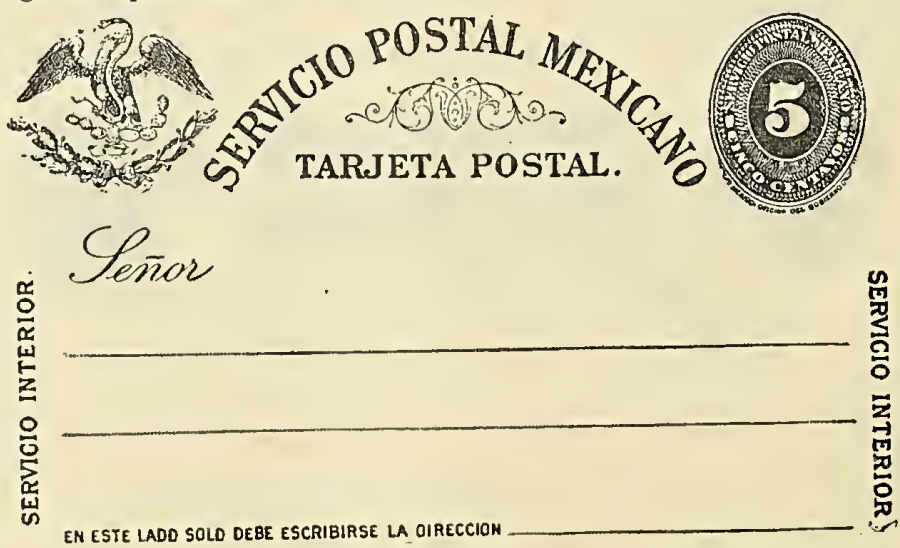

$40485 \mathrm{c}$ blue, carmine impression on white card.

a. No period after INTERIOR at right.

b. Period after INTERIOR at right.

c. Stamp inverted in lower left corner.

d. Stamp inverted in upper right corner.

f. Stamp embossed without color.

g. Without stamp.

October, I8go.

Typographed in color, on white card; stamp embossed in upper right corner. Size I4IxgI $\mathrm{mm}$.

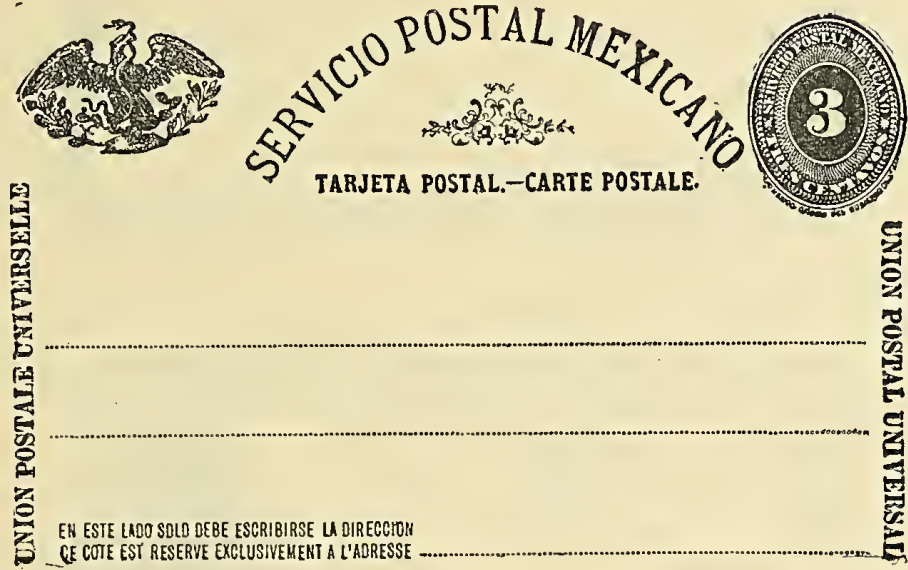

4049 2c carmine, green impression on white card.

a. The two lines for address begin before $s$ and $U$ at the left, and os and $U N$ at the right. Round period above line after CARD POSTALE; no period after direccion.

b. The two lines for address begin before os and $\mathrm{EU}$ at the left, and before $s$ and $U$ at the right. J of TARJETA is smaller than the other letters POSTALE under ornament, and the P away from the rest of the word. Square period after CARTE POSTALE; period after direccion.

$40503 \mathrm{c}$ vermilion, green impression on white card.

a. Same as 4049 a.

b. Same as 4049 b.

c. Stamp inverted on reverse of card.

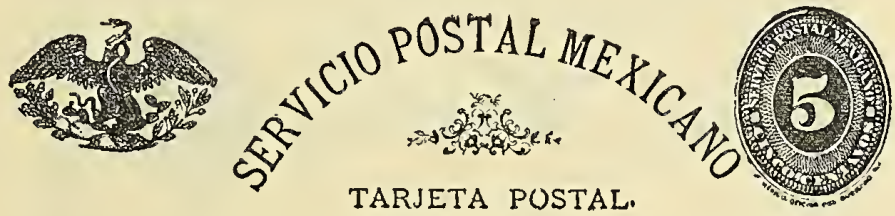

Señar

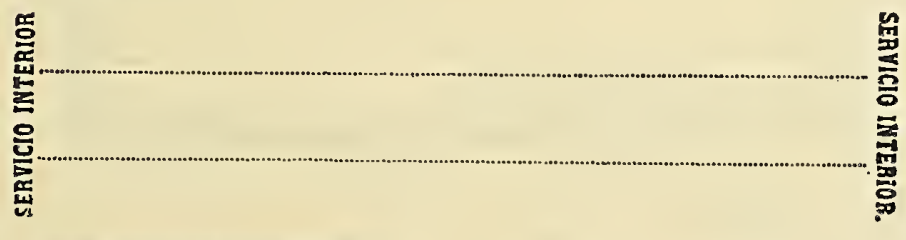

EN ESTE UADO SQLD DEGE ESCRIBIRBE LA DIRECQION.

$405 \mathrm{I} 5 \mathrm{c}$ blue, red impression on white card.

a. The two lines for address begin before $\mathrm{CI}$ and $\mathrm{R}$ at the left and $\mathrm{IC}$ and $\mathrm{E}$ at the right.

$\mathrm{b}$. The $t$ wo lines for address begin before $I C$ and $\mathrm{R}$ at the left and $\mathrm{VI}$ and $\mathrm{E}$ at the right.

c. Same as a, with inscriptions in light red.

d. Same as b, with inscriptions in light red. 
July, I89I.

Typographed in color on white card; stamp embossed in upper right corner. Size $142 \times 90 \mathrm{~mm}$.

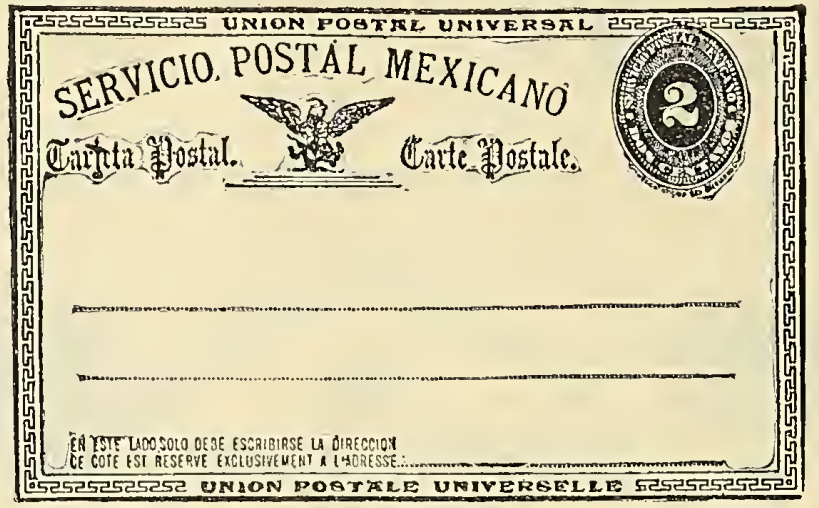

4052 2c carmine, green impression on white card.

$40533 \mathrm{c}$ vermilion, green impression on white card.

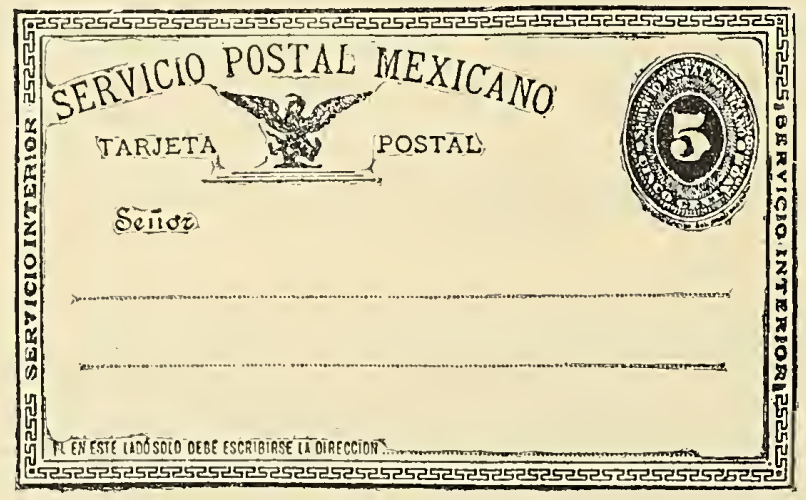

$40545 \mathrm{c}$ blue, red impression on white card.

August, I89r.

Typographed in color on white card ; stamp embossed in upper right corner. Size $142 \times 90 \mathrm{~mm}$

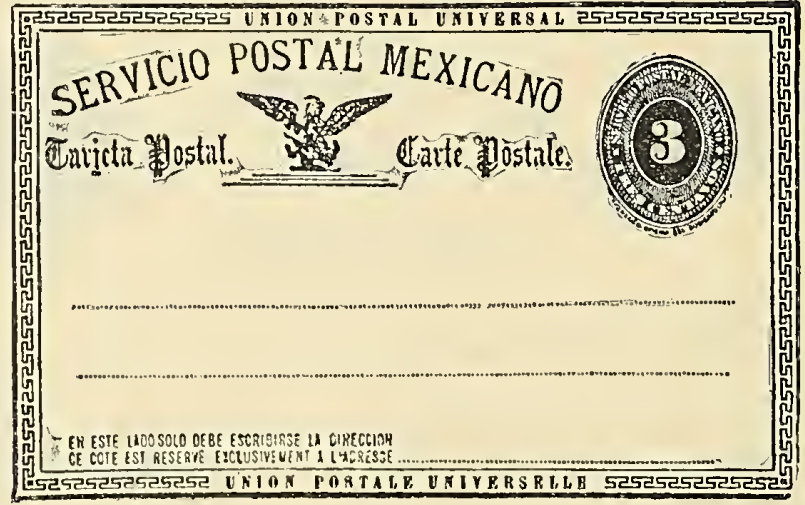

$40552 \mathrm{c}$ carmine, green impression on while card.

a. Yellow green impression.

b. Blue green impression.

c. Yellow green impression and stamp on face, and blue green without stamp on reverse of card.

d. Stamp inverted in lower left corner.

$40563 \mathrm{c}$ vermilion, red impression on white card.
a. Red impression on white card.
b. Carmine impression on bluish white card.
c. Rose impression on bluish white card.
d. Rose impression on cream card.
e. Carmine impression on pale rose card.
f. Stamp inverted. 


$$
\text { . }
$$



November, 1892 .

Typographed in red on white card. Size $138 \times 92 \mathrm{~mm}$.

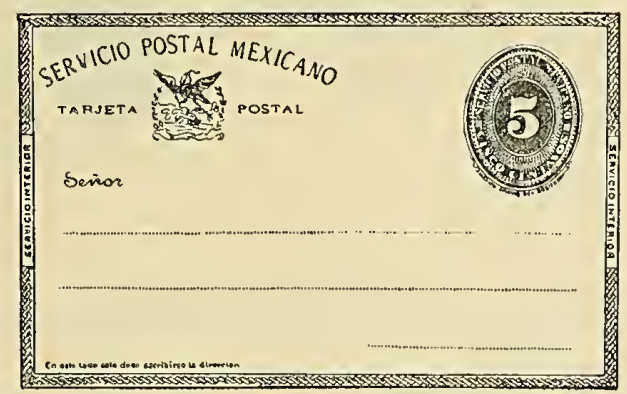

$\$ 0575 \mathrm{c}$ blue, red impression on white card.

Lithographed in color on cards of various colors; stamp embossed in upper right corner. Size $138 \times 7 \mathrm{r} \mathrm{mm}$
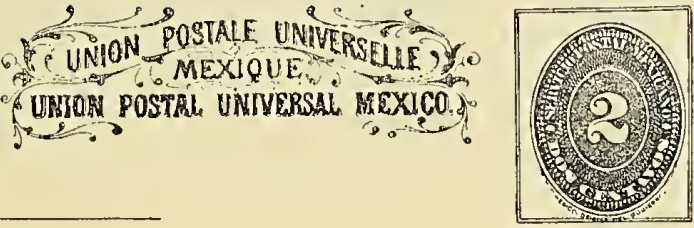

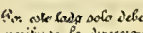

$40582 \mathrm{c}$ carmine, rose impression on various colored cards.
a. Rose card.
b. Green card.
c. Blue green card.
d. Lavender card.
e. Yellow card.
f. Gray card.

4059 2c carmine, blue impression on blue green card.

$40602 \mathrm{c}$ carmine, black impression on reddish buff card,

406I 2c carmine, carmine impression on lavender card.

January, 1893 .

Typographed in green on white card. Size I 4 Ix90 $\mathrm{mm}$.

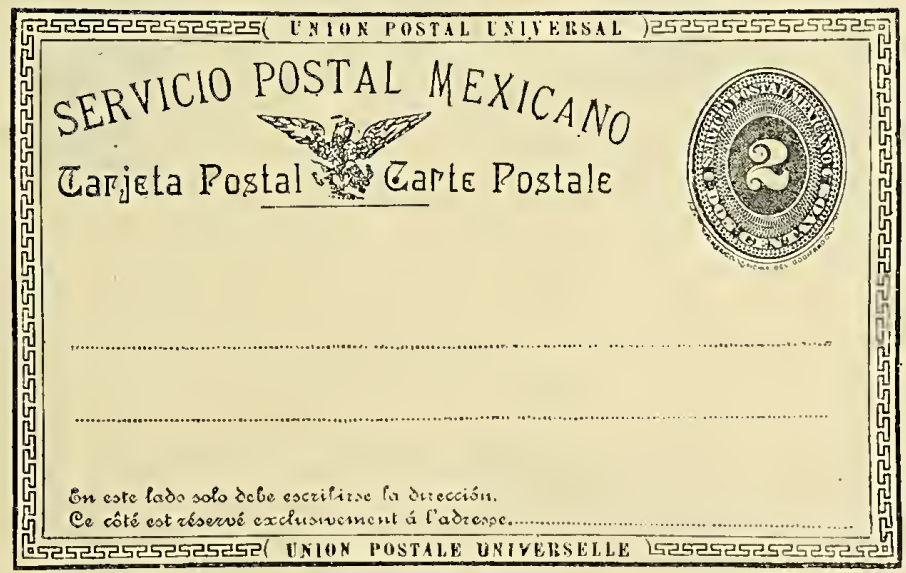

$40622 \mathrm{c}$ carmine, green impression on white card.
a. Bluish wohite card.
b. Rosy white card.
c. Stamp embossed on the extreme right side of the card, so that only a part of it can be seen.
d. Without stamp. 
Typographed in carmine on white card; stamp embossed in upper right corner. Size $140 x 90 \mathrm{~mm}$.

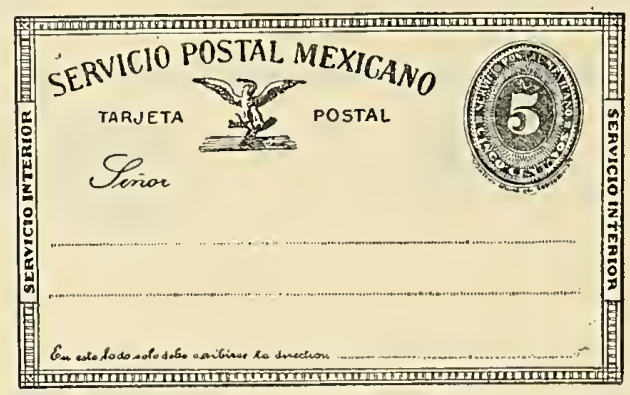

$40635 \mathrm{c}$ blue, carmine impression on zwite card.

July, I893.

Typographed in green on white card; stamp embossed in upper right corner. Size $137 \times 90 \mathrm{~mm}$.

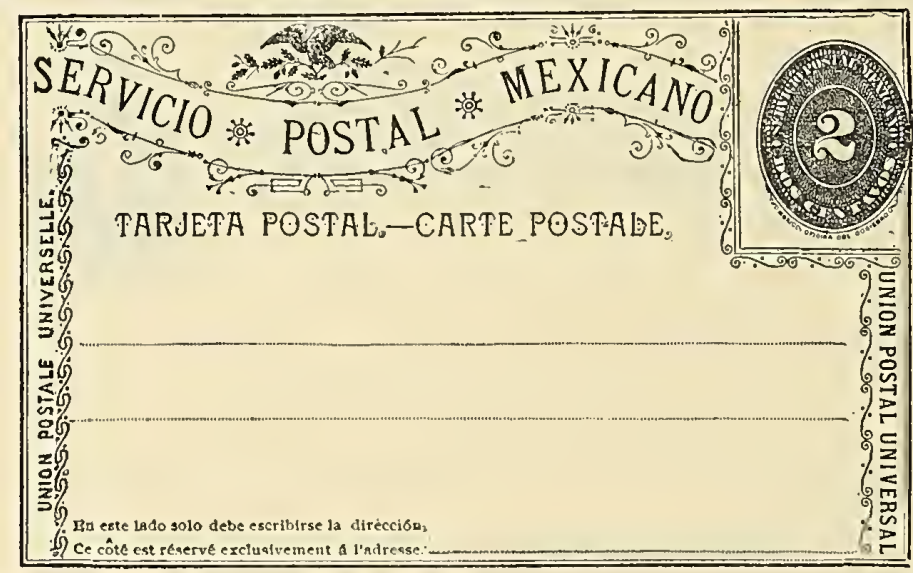

$40642 \mathrm{c}$ red, green impression on white card.

a. Stamp correct

b. Stamp inverted.

c. Stamp sideways in upper right corner of card.

$40653 \mathrm{C}$ vermilion, green impression on white card.

$40665 \mathrm{c}$ blue, green implession on white card.

April, I894.

Typographed in color on various cards. Size $139 \times 90 \mathrm{~mm}$.

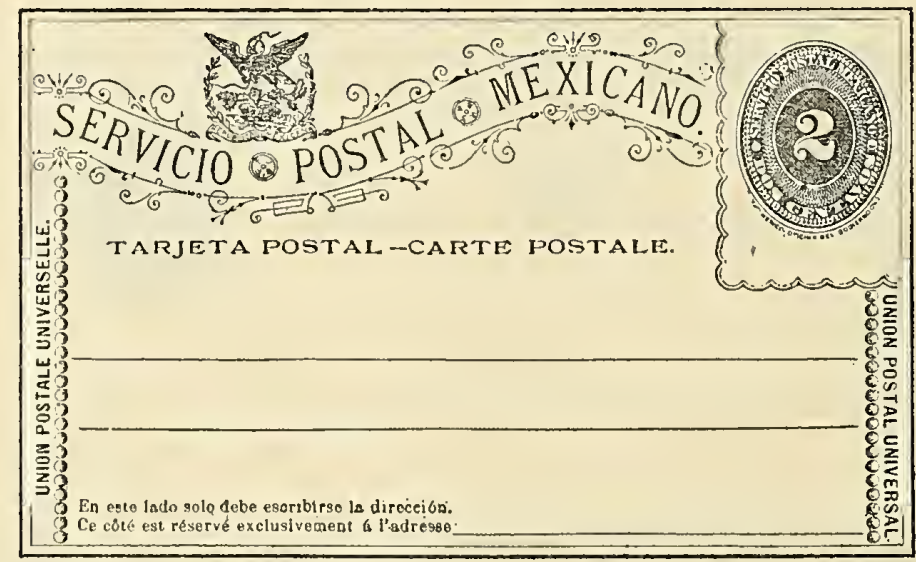

First o of solo without accent.

4067 2c scarlet, green impression on buff card.

a. Blue green impression.

b. Yellow green impression.

$40683 \mathrm{c}$ vermilion, green impression on buff card.

a. Blue green impression.

b. Yellow green impression. 


$40695 \mathrm{c}$ blue, rose impression on buff card.

Similar to preceding, but accent over first o of solo. The ornament under the $\mathrm{x}$ of MEXICANo has same shape as the one above it; the scroll at the left of the eagle is turned towards the right instead of left. $40702 \mathrm{c}$ scarlet, green impression on buff card.

a. Blue green impression.

b. Yellow green impression.

4071 3c vermilion, green impression on buff card.

a. Blue green impression.

b. Yellow green impression.

$40725 \mathrm{c}$ blue, rose impression on buff card.

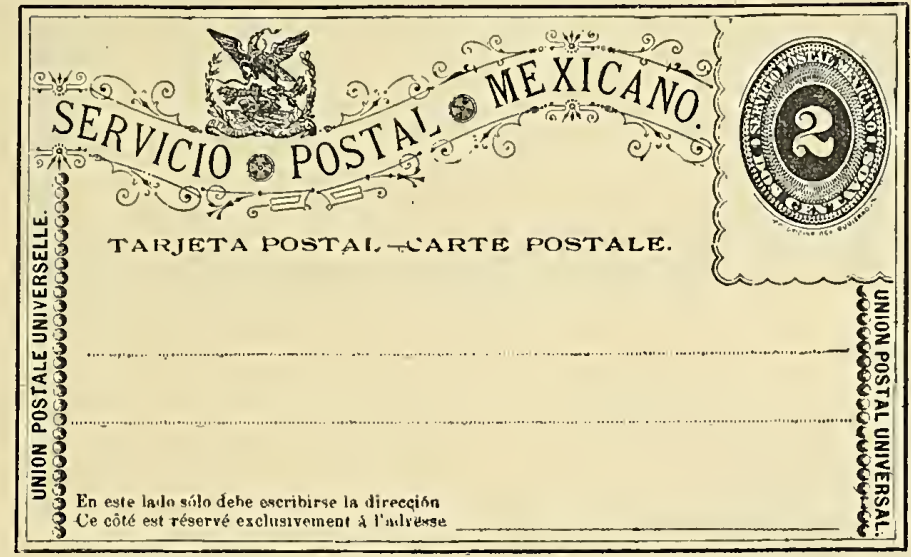

4073 2c scarlet, green impression on buff card.

a. Blue green impression.

b. Yellow green impression.

$40743 \mathrm{c}$ vermilion, green impression on buff card.

a. Blue green impression.

b. Yellow green impression.

$40755 \mathrm{c}$ blue, rose impression on buff card.

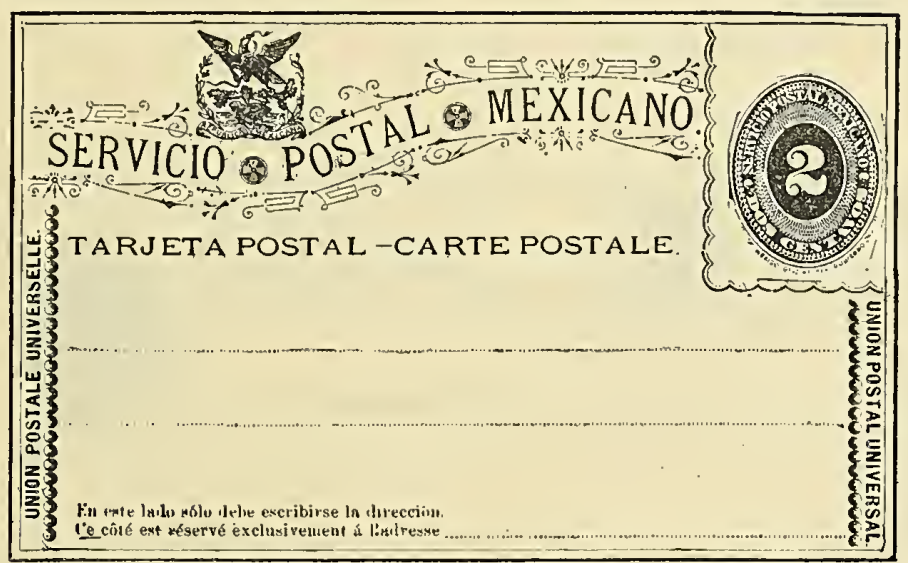

First I of direccion without dot.

4076 2c scarlet, green impression on buff card.

a. Blue green impression.

b. Yellow green impression.

4077 3c vermilion, green impression on buff card.

a. Blue green impression.

b. Yellow green impression.

$40785 \mathrm{c}$ blue, rose impression on buff card.

Similar to preceding, but first I of direccion with dot. The scroll under o of SERvicio and o of MEXICANO is inverted; the ornament under the S of SERvicio has the same shape as the one above it.

4079 2c scarlet, green impression on buff card.

a. Blue green impression.

b. Yellow green impression.

$40803 \mathrm{c}$ vermilion, green impression on buff card.

a. Blue green impression.

b. Yellow green impression.

$408 \mathrm{I} 5 \mathrm{C}$ blue, rose impression on buff card. 


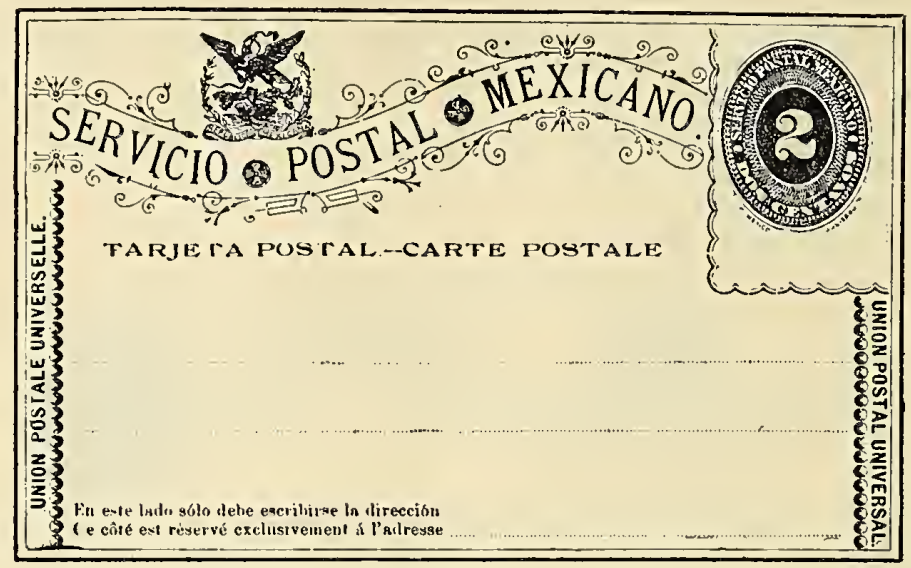

The I of exclusivement is without dot.

4082 2c scarlet, green impression on buff card.

a. Blue green impression.

b. Yellow green impression.

$40833 \mathrm{c}$ vermilion, green impression on buff card.

a. Blue green impression.

b. Yellow green impression.

$40845 \mathrm{c}$ blue, rose impression on buff card.

Similar to preceding, but dot over I of exclusivement.

4085 2c scarlet, green impression on buff card.

a. Blue green impression.

b. Yellow green impression.

$40863 \mathrm{c}$ vermilion, green impression on buff card.

a. Blue green impression.

b. Yellow impression.

$40875 \mathrm{c}$ blue, rose impression an buff card.

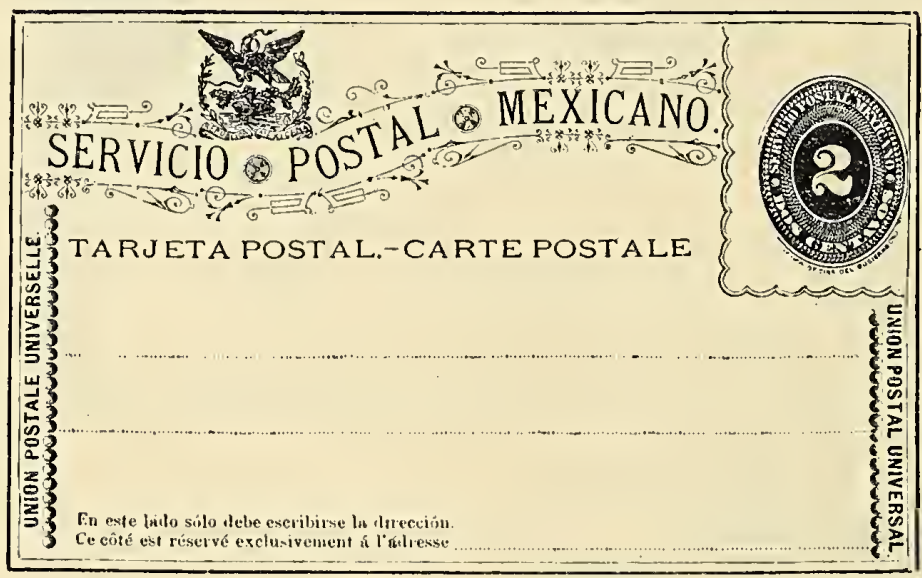

4088 2c scarlet, green impression on buff card.

a. Blue green impression.

b. Yellow green impression.

$40893 \mathrm{c}$ vermilion, green impression on buff card

a. Blue green impression.

b. Yellow green impression.

$40905 \mathrm{c}$ blue, rose impression on buff card. 

July, I894.

Typographed in red on white card; stamp embossed in upper left corner. Size I3IX8I mm.

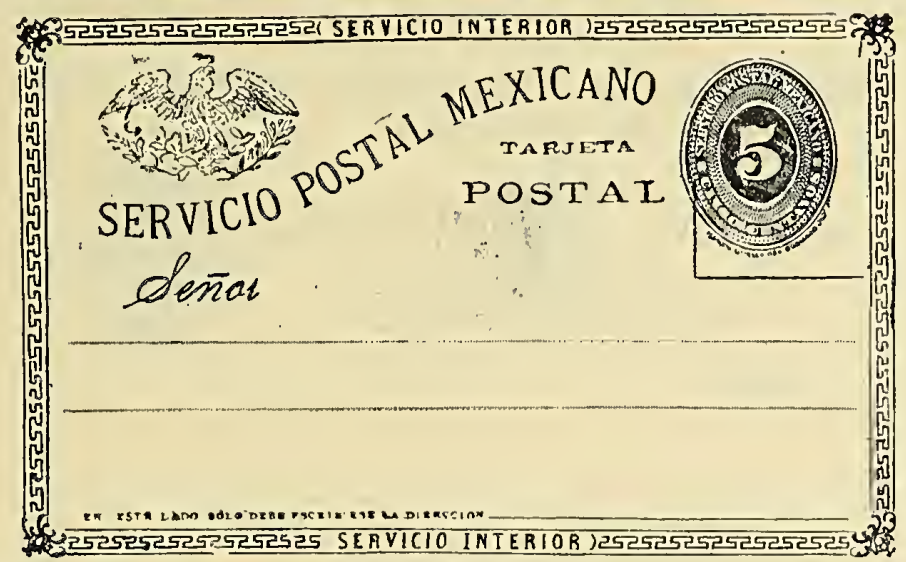

409 I $5 \mathrm{c}$ blue, red impression on white card.

a. $9^{\text {th }}$ ornament from the left, in frame at top, a little above the others.

b. $9^{\text {th }}$ ornament from the left at top inverted.

c. 9th ornament inverted and a dash under SER of SERVICIO.

Typographed in red on white card; stamp embossed in upper right corner. Size I3IX8I mm

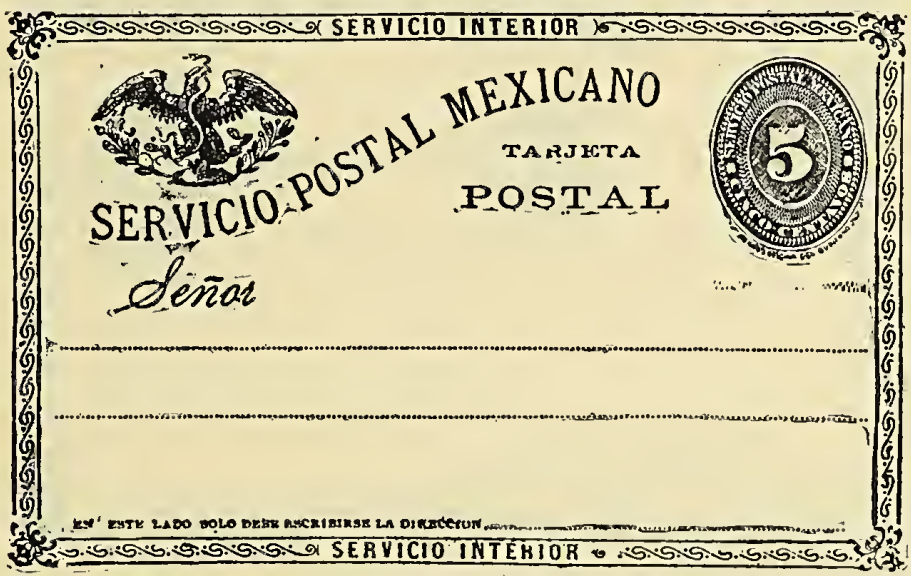

$40925 \mathrm{c}$ blue, red impression on white card.

a. Instructions at bottom correct.

b. EN ESF instead of EN ESTE.

January, 1895 .

Typographed in rose on buff card; stamp impressed in upper right corner. Size 131x81 mm.

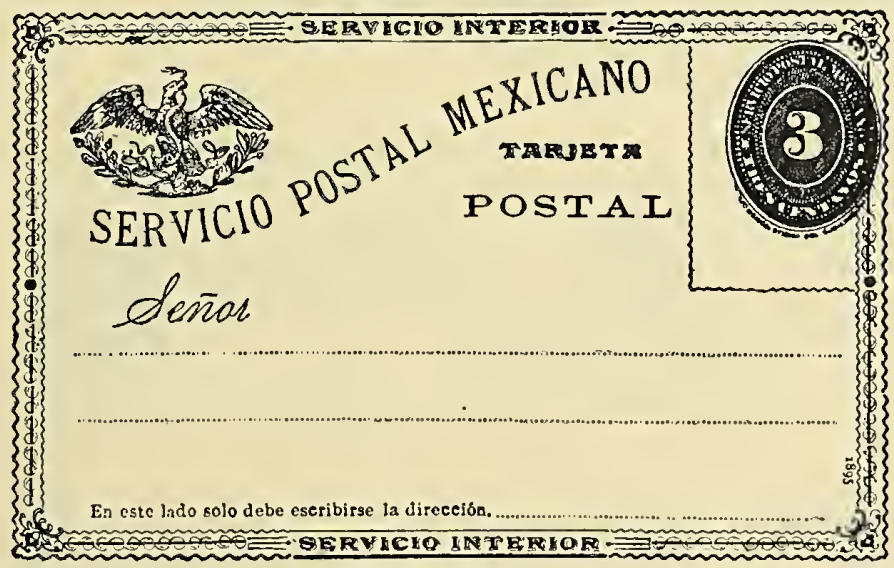

4093 3c vermilion, rose impression on buff card
a. Stamp scarlet, frame rose.
b. Stamp vermilion, frame pale rose.
c. Stamp vermilion, frame rose.
d. Stamp pale vermilion, frame dark rose. 
February, 1895.

Provisional Issue.

Typographed in color on buff card, Adhesive stamp of current issue pasted in upper right corner. Size I4IX91 mm.

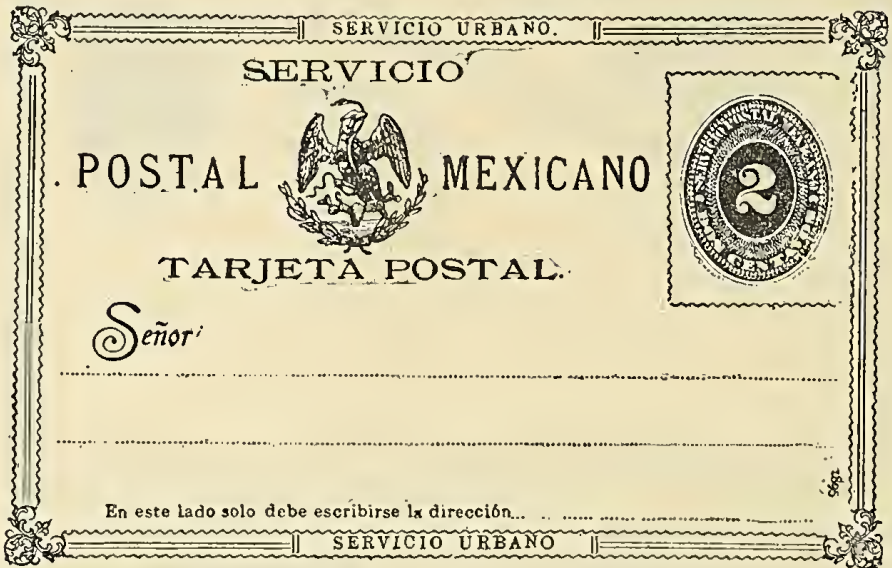

4094 2c carmine, rose impression on buff card.

b. Stamp vermilion.

c. Without stamp.

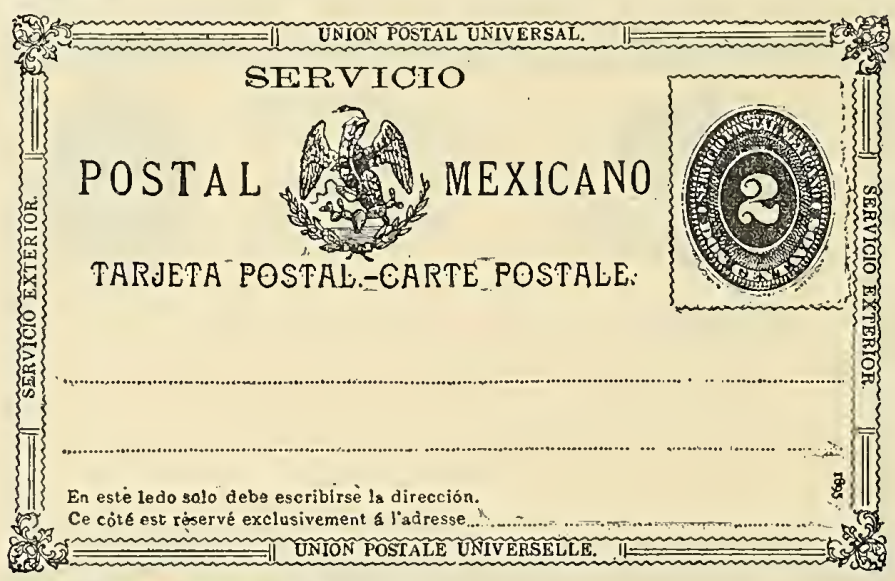

4095 2c vermilion, green impression on buff card.

a. Stamp scarlet.

b. Stamp vermilion, frame light green.

$40963 \mathrm{c}$ scarlet, green impression on buff card.

March, I895.

Provisional issue.

Same types as preceding issue. Typographed in color on white card.

$40972 \mathrm{c}$ carmine, rose impression on white card.

4098 2c carmine, green impression on white card.

$40993 \mathrm{c}$ scarlet, green impression on white card.

April, I895.

Typograpled in color on white and buff card; stamp embossed in upper right corner. Size $139 \times 88 \mathrm{~mm}$ c. Stamp vermilion, frame dark green.

d. Without stamp.

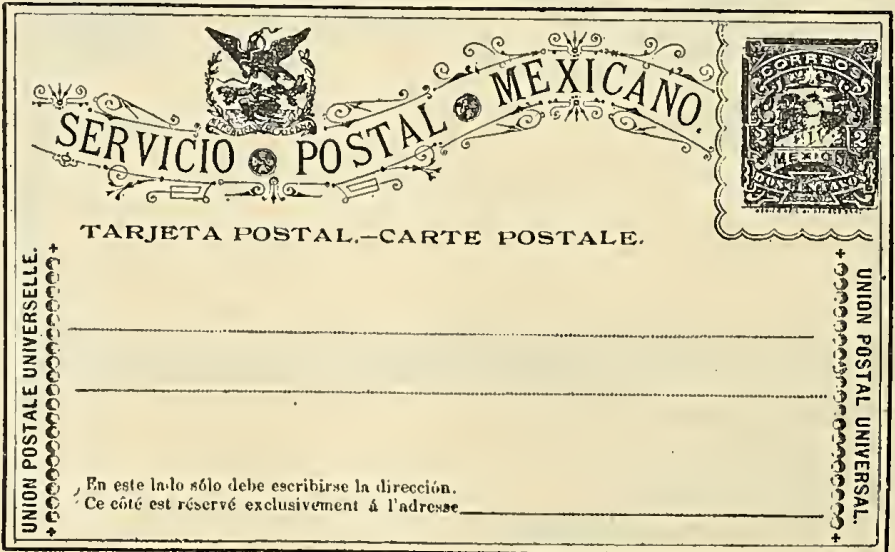



Servicio interior at sides; tARJETA POStal $46 \mathrm{~mm}$. long.

4100 $2 \mathrm{c}$ rose, rose impression on various cards.

a. On white card.

b. On buff card.

4 IOI $3 \mathrm{C}$ brown, rose impression on various cards.

a. On white card.

b. On buff card.

UNION etc. at sides; TARJETA etc. $93 \mathrm{~mm}$. long.

$4 \mathrm{IO} 22 \mathrm{c}$ rose, green impression on buff card.

4ro3 $3 \mathrm{c}$ brown, green impression on buff card.

SERVICIO INTERIOR at sides ; TARJETA POSTAL 4I mm. ong.

$41042 \mathrm{c}$ rose, rose impression on various cards

a. On white card.

b. On buff card.

$41053 \mathrm{c}$ brown, rose impression on various cards.

a. On white card.

b. On buff card.

UNION etc at sides; TARJETA etc. $85 \mathrm{~mm}$. long.

$41062 \mathrm{c}$ rose, green impression on buff card.

$41073 \mathrm{c}$ brown, green impression on buff card.

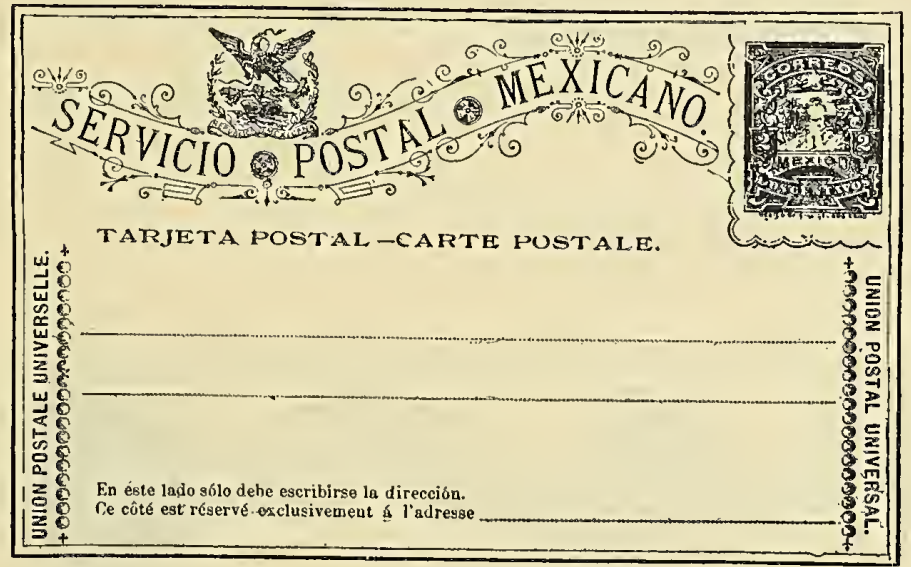

SERVICIO INTERIOR at side; TARJETA POSTAL $46 \mathrm{~mm}$, long.

$41082 \mathrm{c}$ rose, rose impression on various cards.
a. On white card.

b. On buff card.

4109 3c brown, rose impression on various cards.

a, On white card.

b. On buff card.

UNION etc. at sides ; TARJETA etc. $93 \mathrm{~mm}$. long.

$4 \mathrm{IIO} 2 \mathrm{c}$ rose, green impression on buff card.

$4 \mathrm{III} 3 \mathrm{c}$ brown, green impression on buff card.

SERVICIO INTERIOR at sides; TARJETA POSTAL 4I mm. long.

$4 \mathrm{Ir2} 2 \mathrm{c}$ rose, rose impression on various cards.

a. On white card.

b. On buff card.

$4 \mathrm{II}_{3} 3 \mathrm{c}$ brown, rose impression on various cards.

a. On white card.

b. On buff card.

UNION etc. at sides; TARJETA etc. $85 \mathrm{~mm}$. long.

$4 I 142 c$ rose, green impression on buff card.

$41153 \mathrm{c}$ brown, green impression on buff card.

Same as $4 I I 2$ to $4 I I 5$, but ornament under $x$ of MEXICANO differs from the one above it.

4 II6 2c rose, rose impression on various cards.

a. On white card.

b. On buff card.

$4 \mathrm{II} 73 \mathrm{c}$ brown, rose impression on various cards.

a. On white card.

b. On buff card.

$4 \mathrm{II} 8$ 2c rose, green impression on buff card.

4 II9 3C brown, green impression on buff card. 


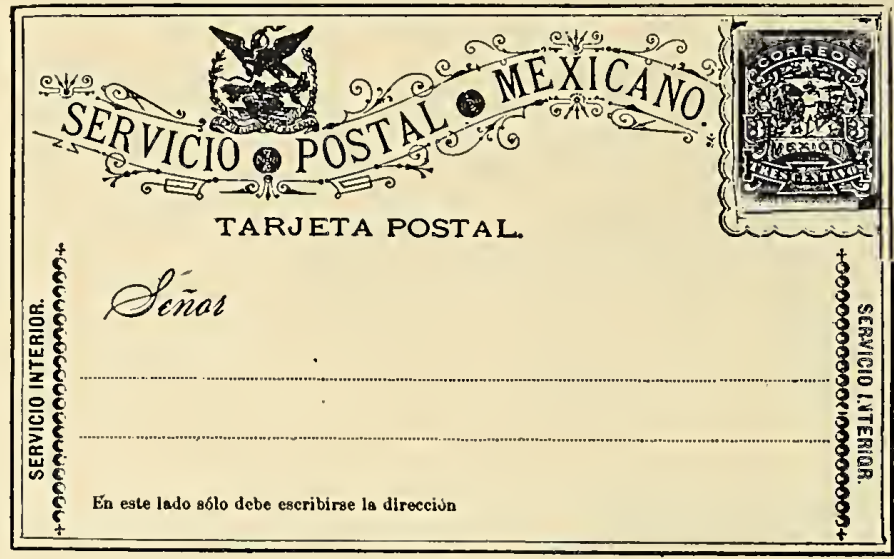

SERVICIO INTERIOR at sides; TARJETA POSTAL $46 \mathrm{~mm}$. long.

$2 c$ rose, rose impression on various cards.

a. On white card.

b. On buff card.

3c brown, rose impression on various cards,

a. On white card.

b. On buff card,

UNION etc. at sides; TARJETA etc. $93 \mathrm{~mm}$. long.

4 I 22 2c rose, green impression on buff card.

$4^{\mathrm{I} 23} 3 \mathrm{C}$ brown, green impression on buff card.

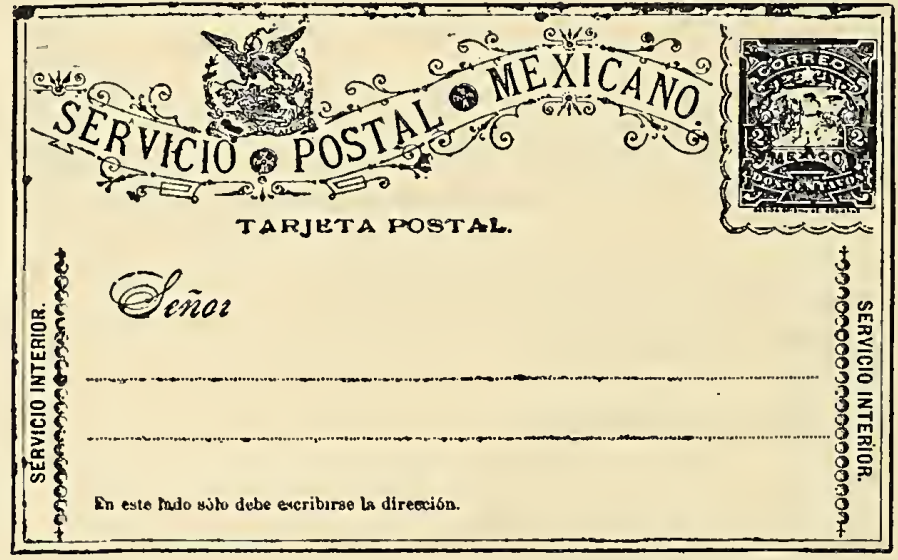

SERVICIO INTERIOR at sides; TARJETA POSTAL 4 I $\mathrm{mm}$. long.

c rose, rose impresion on various cards.

a. On white card.

b. On buff card.

3c brown, rose impression on various cards.

a. On wolite card.

b. On buff card.

UNION etc. at sides; TARJETA etc. $85 \mathrm{~mm}$. long.

4I 26 2c red, green impression on buff card.

$41273 \mathrm{c}$ brown, green impression on buff card.

Same as $4 \mathrm{I} 24$ to $4 \mathrm{I} 27$, but first dotted line shorter than second one.

$41282 \mathrm{c}$ rose, rose impression on various cards.

a. On white card.

b. On buff card.

4.29 3c brown, rose impression on various cards

a. On white card.

b. On buff card.

$4 \mathrm{I} 302 \mathrm{c}$ rose, green impression on buff card.

4I3I 3c brown, green impression on buff card. 


November, 1884 .

\section{LETTER CARDS.}

Lithographed in black on white card; stamp impressed in upper right corner. Size $142 \times 92 \mathrm{~mm}$.

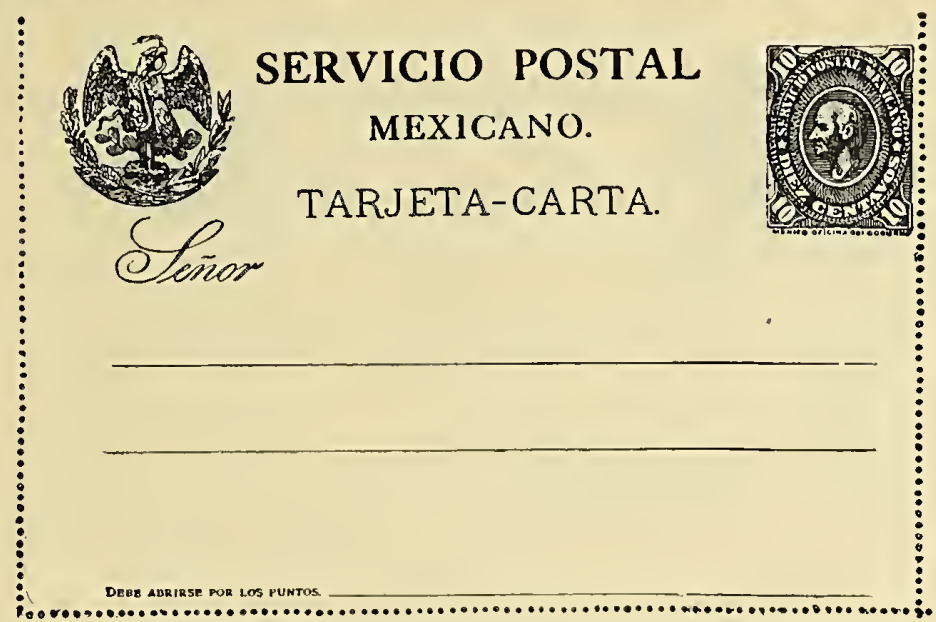

450 I IOc green, black impression on white card.

a. Vertical perforations $136 \mathrm{~mm}$. apart.

b. Vertical perforations $139 \mathrm{~mm}$. apart.

c. Vertical perforations $\mathrm{I} 4 \mathrm{I} \mathrm{mm}$. apart.

February, I 886 .-

Lithographed in black on white card; stamp impressed in upper right corner. Size $142 \times 92 \mathrm{~mm}$

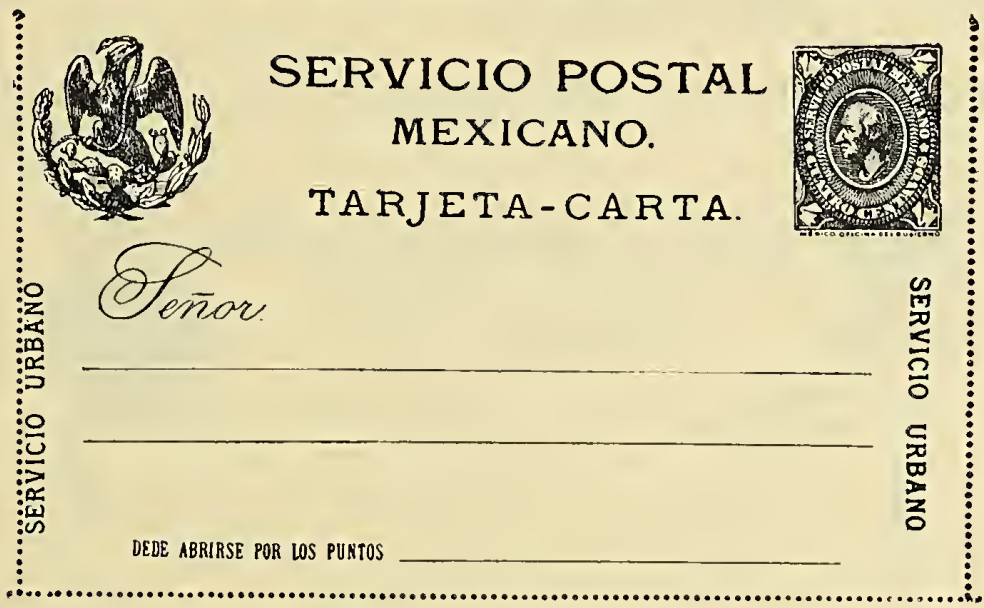

$45024 \mathrm{c}$ vernilion, black impression on white card.

a. On thick white card, vertical perforations $137 \mathrm{~mm}$ apart.

b. On thin bluish card, vertical perforations $137 \mathrm{~mm}$. apart.

c. On thick white card, vertical perforations $139 \mathrm{~mm}$. apart.

d. On thick white card, vertical perforations $\mathrm{I} 4 \mathrm{I} \mathrm{mm}$. apart.

e. On thin bluis $h$ card, double perforations at bottom $138 \mathrm{~mm}$. apart.

f. On thick white card, double perforations at bottom $138 \mathrm{~mm}$. apart.

g. On thin bluish card, perforations cut inscriptions at right.

$\mathrm{h}$. On thin bluish card, double perforations at sides.

Same type as issue of November, 1884. Lithographed in black on white card.

4503 IOc orange, black impression on white card.
a. Vertical perforations $134 \mathrm{~mm}$. apart.
b. Vertical perforations I $36 \mathrm{~mm}$. apart.
c. Vertical perforations $138 \mathrm{~mm}$. apart.
d. Vertical perforations $140 \mathrm{~mm}$. apart.
e. Vertical perforations I I I mm. apart.
f. Double perforations at left side.
g. Perforations cut inscriptions at bottom.
h. Perforations cut inscriptions at left side.
i. Perforations cut inscriptions at right side.
j. Without stamp. Perforations $140 \mathrm{~mm}$. apart. 
January, I 890.

Lithographed in black on buff card; stamp impressed in upper right corner. Size $142 \times 92 \mathrm{~mm}$.

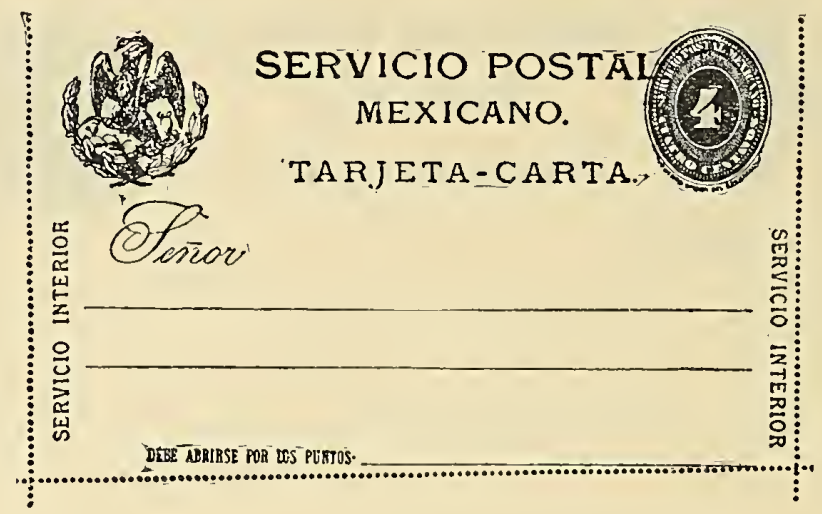

$45044 \mathrm{c}$ scarlet, black impression on buff card. SERVICIO URBANO at sides.

$45054 \mathrm{c}$ scarlet, black impression on buff card. SERVICIO INTERIOR at sides.

a. Double perforations at right.

b. Double perforations at bottom.

c. No perforations at left.

d. Double perforations at left; no perforations at bottom.

e. Inscription at bottom is cut by perforations.

f. Inscription at left is cut by perforations.

g. Inscription at right is cut by perforations.

March, I 890 .

Lithographed in black on buff card; stamp impressed in upper right corner. Size $148 \times 92 \mathrm{~mm}$.

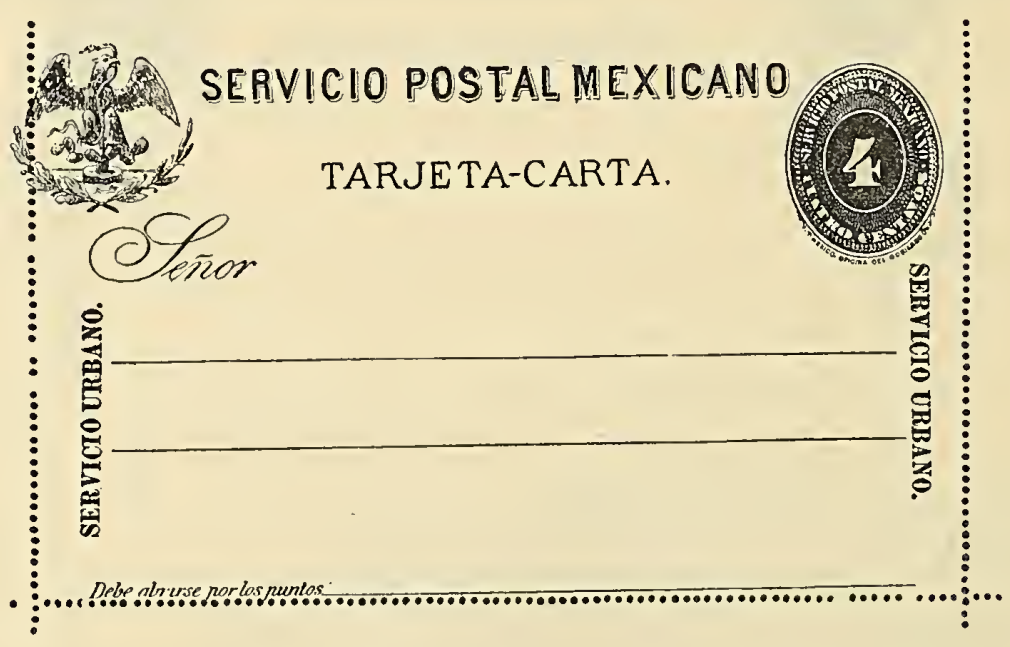

$45064 \mathrm{c}$ scarlet, black impression on buff card.

a. Vertical perforations $135 \mathrm{~mm}$. apart.

b. Vertical perforations $140 \mathrm{~mm}$. apart.

c. Double perforation at bottom.

d. Perforalions cut instructions at bottom.

e. Stamp without color.

f. Without instructions at bottom.

g. Stamp on reverse of card.

4507 IOc scarlet, black impression on rose card.

a. Card correct.

b. Instructions at bottom cut by perforations.

4508 Ioc scarlet, black impression on white card,

It is said that only 86 of these last cards were issued. 
April, I895.

Lithographed in blue or black on white card; stamp imptessed in color in upper right corner. Size $4 \mathrm{I} 5 \mathrm{xg0} \mathrm{mm}$.

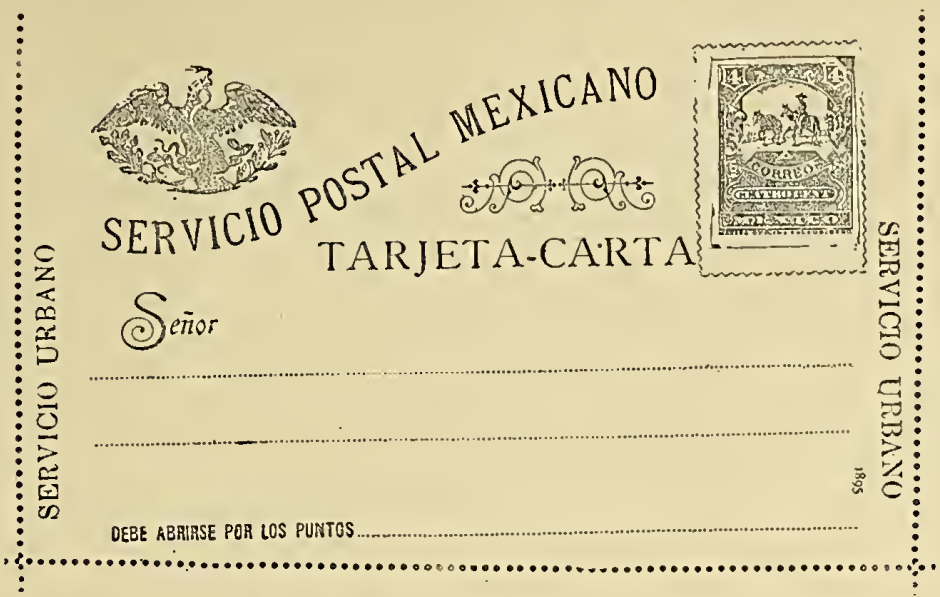

$45094 \mathrm{c}$ orange, blue impression on white card.

a. Very light blue impression; vertical perforations $139 \mathrm{~mm}$. apart.

b. Blue impression, vertical perforations $139 \mathrm{~mm}$. apart.

c. Dark blue impression, vertical perforations $139 \mathrm{~mm}$. apart.

d. Blue impression, vertical perforations I $36 \mathrm{~mm}$. a part.

e. Dark blue impression with additional inscription,"Abrisse por los puntos," across lower left corner 45 ro IOc violet, black impression on white card.

a. Perforations correct.

b. Double perforation at right side.

c. Double perforation at left side.

OFFICIAL CARDS.

January, I 885 .

Lithographed in color on white card.

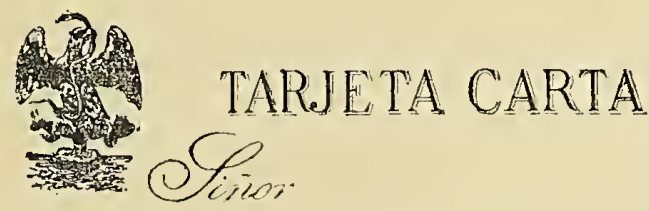

D. Be ubinow por kwo purtho.

4701 No value, impression in various colors on white card.
a. Blue impression.
b. Green impression.
c. Carmine impression. 


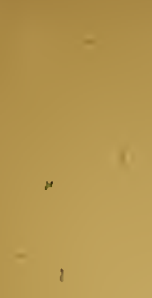

*

1.

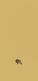

: 



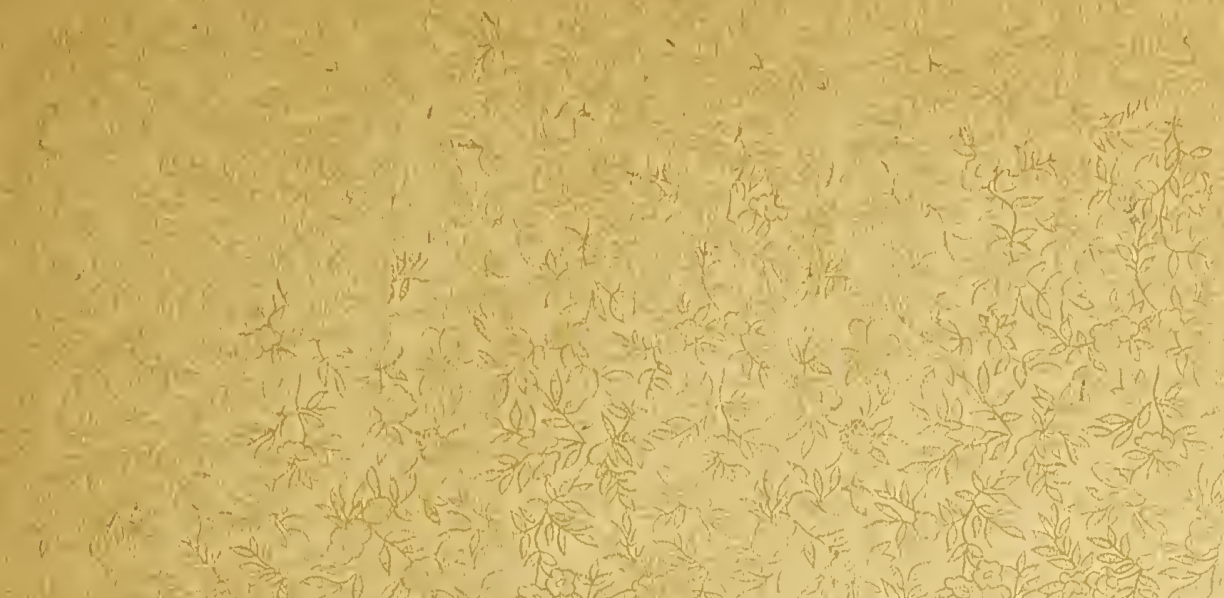

$$
\rightarrow \text { ito }
$$

$\prod_{1}$
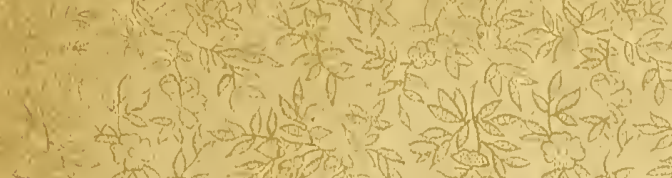

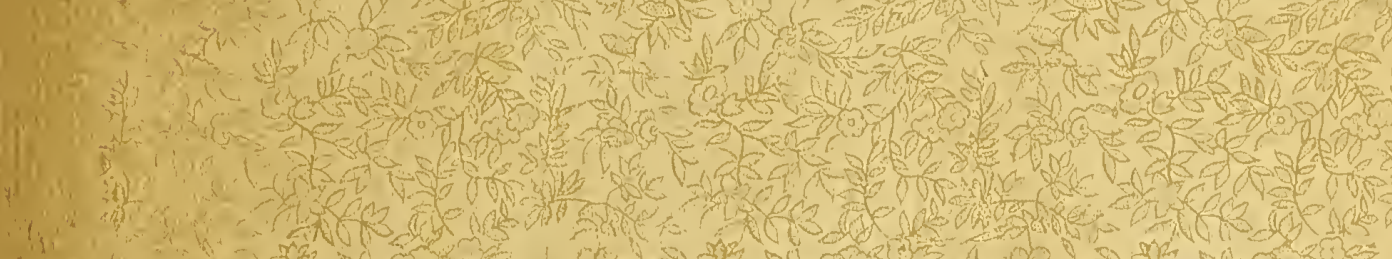

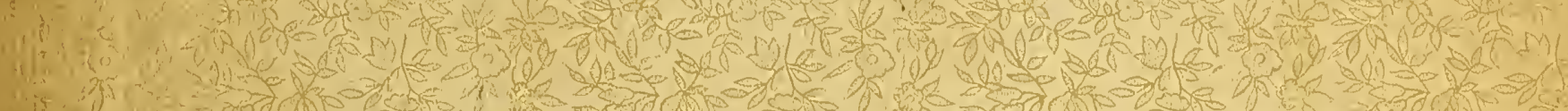

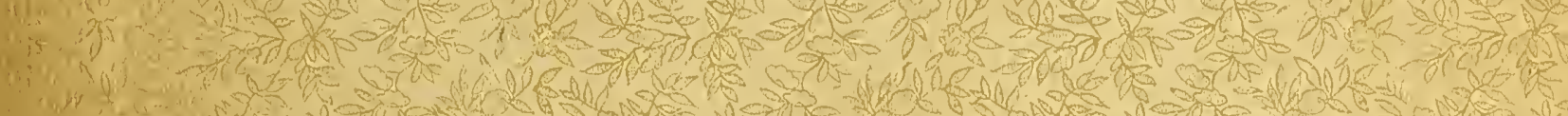

4 m

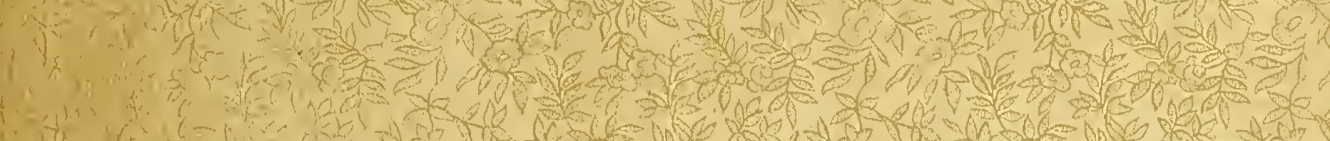

4) $\quad$ -

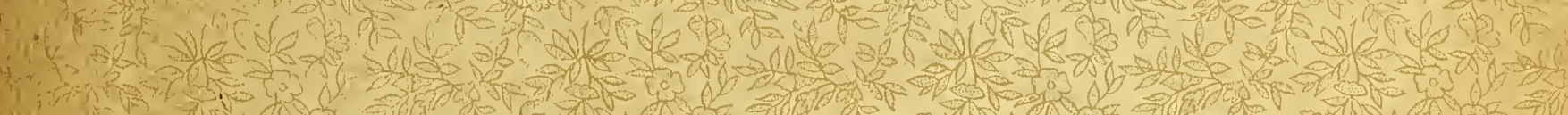

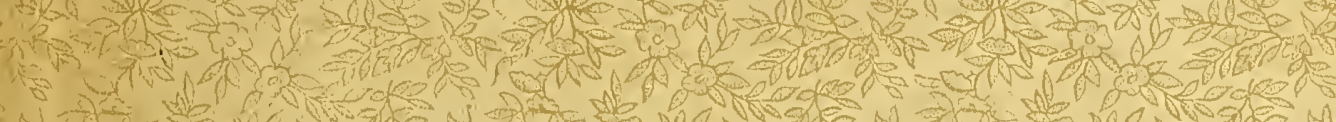
149 -

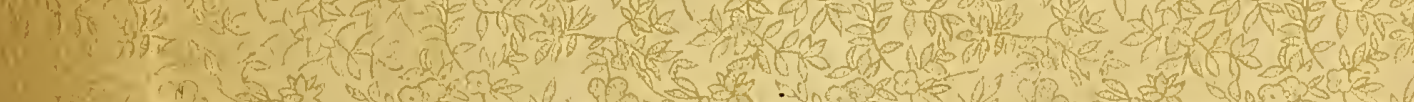

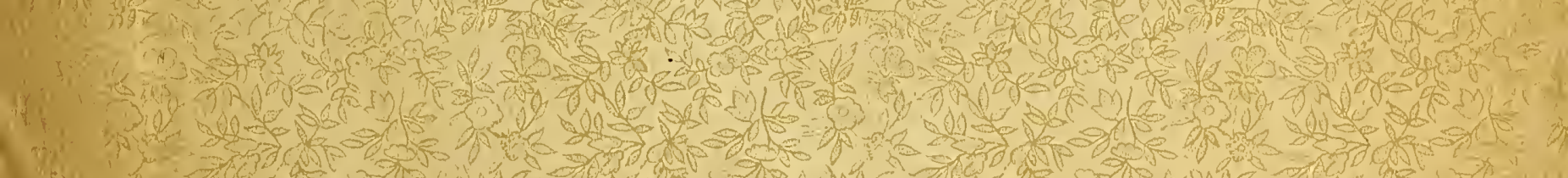

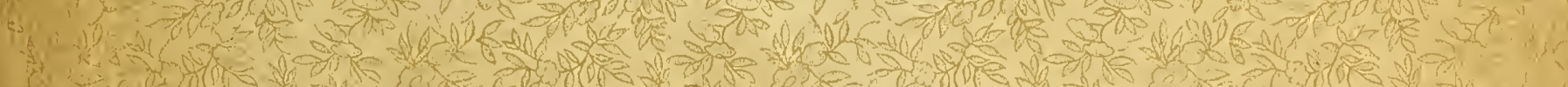

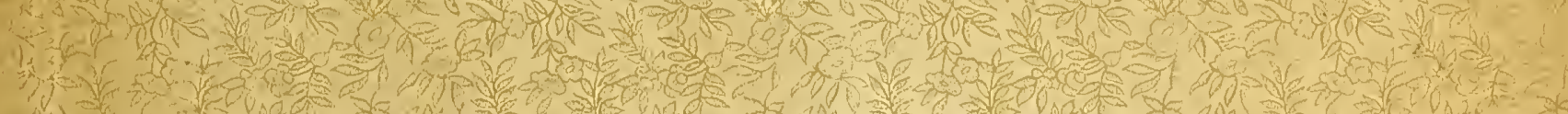
Ti 1. - A

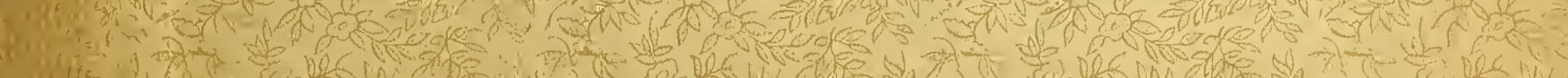
(19) 
